Supporting Information For

\author{
Asymmetric Hydrogenation of $\beta$-Aryl Alkylidene Malonate Esters: Installing an Ester Group \\ Significantly Increases the Efficiency \\ Qiankun Zhao, Xiong Wu, Lin-Ping Li, Fan Yang, Jian-Hua Xie*, Qi-Lin Zhou
}

State Key Laboratory and Institute of Elemento-organic Chemistry, College of Chemistry, Nankai

University, Tianjin 300071, China

Contents

General: .S1

(A) Preparation of New SpiroPAP Ligands and Their Catalysts.....................................S2

(B) Preparation of $\boldsymbol{\beta}$-Aryl Alkylidene Malonate Esters.............................................S7

(C) Asymmetric Hydrogenation of $\beta$-Aryl Alkylidene Malonate Esters............................S13

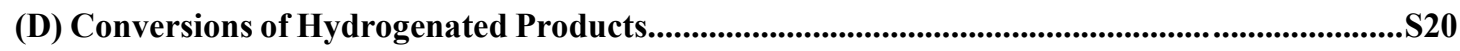

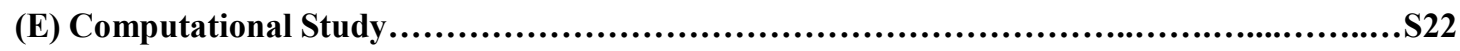

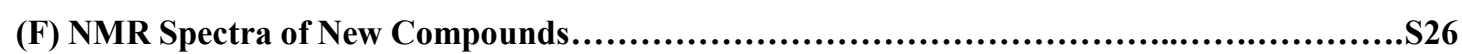

(G) HPLC Charts for Hydrogenation Products....................................................S89

(H) Cartesian Coordinates and Energies of the Optimized structures.............................S111

\title{
General:
}

All reactions and manipulations which are sensitive to moisture or air were performed under an inert atmosphere of nitrogen. All chemicals were purchased from J\&K, Acros and Aldrich and were used as received. Hydrogen gas (99.999\%) was purchased from Boc Gas Inc., Tianjin. Anhydrous THF was distilled from sodium benzophenone ketyl. Anhydrous $\mathrm{CH}_{2} \mathrm{Cl}_{2}$ and $\mathrm{Et}_{3} \mathrm{~N}$ were freshly distilled from calcium hydride. Anhydrous EtOH was freshly distilled from magnesium. Melting points were measured on a RY-I apparatus and uncorrected. ${ }^{1} \mathrm{H}$ NMR spectra were recorded at $400 \mathrm{MHz}$ on Bruker AV 400 spectrometer. ${ }^{13} \mathrm{C}$ NMR spectra were recorded at $100 \mathrm{MHz}$ on Bruker AV 400 spectrometer. NMR spectra were recorded in deuterated chloroform $\left(\mathrm{CDCl}_{3}\right)$ as a solvent, with residual chloroform $(\delta: 7.26$ ppm. for ${ }^{1} \mathrm{H}$ NMR and $\delta: 77.00 \mathrm{ppm}$. for ${ }^{13} \mathrm{C} \mathrm{NMR}$ ) or tetramethylsilane (TMS, $\delta: 0.00 \mathrm{ppm}$. for ${ }^{1} \mathrm{H} \mathrm{NMR}$ ) taken as the inert standard, and were reported in ppm. Abbreviations for signal coupling are as follows: s, singlet; d, doublet; t, triplet; q, quartet; m, multiplet. Coupling constants were taken from the spectra directly and are uncorrected. Optical rotations were determined using a Perkin Elmer 341 polarimeter. High Resolution Mass (HRMS) were recorded on Varian 7.0T FTMS using an electrospray (ESI) ionization source. HPLC analyses were performed using Hewlett Packard Model HP1100 instruments with Chiralcel AD-H, IH-3 and IC-3 column. 
(A) Preparation of New SpiroPAP Ligands and Their Iridium Catalysts

\section{Synthesis of 3-substituted picolinaldehydes}

The 3-substituted picolinadehydes $\mathbf{S 3}$ were synthesized as outlined below.<smiles>COC(=O)c1cccnc1C</smiles>

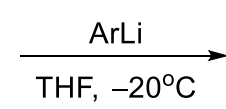
$59-95 \%$

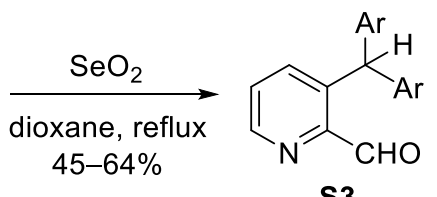

S3<smiles>Cc1ncccc1C(Br)(Br)Br</smiles>

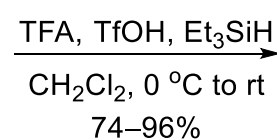
$74-96 \%$<smiles>Cc1ncccc1C(Br)(Br)Br</smiles>

Synthesis of 3-hydroxydiarylmethyl-2-methylpyridines S1.

General procedure: To a solution of methyl 2-methylnicotinate (756 mg, $5.0 \mathrm{mmol}$ ) in anhydrous THF $(35 \mathrm{~mL})$ was added an aryl lithium solution in hexane $(15.0 \mathrm{mmol})$ at $-20^{\circ} \mathrm{C}$. After being stirred at the same temperature for $6 \mathrm{~h}$, the solvents were removed in vacuo. The residue was diluted with $\mathrm{Et}_{2} \mathrm{O}$, washed with a saturated ammonium chloride solution, brine, and dried over $\mathrm{MgSO}_{4}$. The solvents were then removed again in vacuo and purified by flash chromatography with petroleum ether/ethyl acetate $(3: 1)$ as an eluent to give the desired products $\mathbf{S 1}$ as white solids or colorless oils.

\section{(2-Methylpyridin-3-yl)diphenylmethanol (S1a)}<smiles>Cc1ncccc1C(O)(c1ccccc1)c1ccccc1</smiles>

White solid, m.p. $96-97{ }^{\circ} \mathrm{C}, 1.29$ g, 94\% yield. ${ }^{1} \mathrm{H}$ NMR (400 MHz, $\left.\mathrm{CDCl}_{3}\right) \delta: 8.23$ (d, $J=4.8 \mathrm{~Hz}, 1 \mathrm{H}), 7.42-7.13(\mathrm{~m}, 10 \mathrm{H}), 7.07(\mathrm{dd}, J=8.0,1.6 \mathrm{~Hz}, 1 \mathrm{H}), 6.93(\mathrm{dd}, J=8.0$, $4.8 \mathrm{~Hz}, 1 \mathrm{H}), 4.02(\mathrm{~s}, 1 \mathrm{H}), 2.32(\mathrm{~s}, 3 \mathrm{H}) ;{ }^{13} \mathrm{C} \mathrm{NMR}\left(101 \mathrm{MHz}, \mathrm{CDCl}_{3}\right) \delta: 159.0,147.6$, 145.9, 140.4, 136.8, 128.2, 127.7, 127.5, 119.9, 81.9, 25.2. HRMS (ESI) m/z: [M+H] ${ }^{+}$ calcd for $\mathrm{C}_{19} \mathrm{H}_{18} \mathrm{NO} 276.1383$; Found 276.1386.

Bis(3,5-dimethylphenyl)(2-methylpyridin-3-yl)methanol (S1b)<smiles>Cc1cc(C)cc(C(O)(c2cc(C)cc(C)c2)c2cccnc2C)c1</smiles>

A colorless oil and solidified slowly by standing, $1.29 \mathrm{~g}, 78 \%$ yield. ${ }^{1} \mathrm{H}$ NMR (400 $\left.\mathrm{MHz} \mathrm{CDCl}_{3}\right) \delta: 8.36(\mathrm{~d}, J=4.0 \mathrm{~Hz}, 1 \mathrm{H}), 7.04(\mathrm{~d}, J=8.0 \mathrm{~Hz}, 1 \mathrm{H}), 7.00-6.87(\mathrm{~m}$, $3 \mathrm{H}), 6.80(\mathrm{~s}, 4 \mathrm{H}), 3.25(\mathrm{~s}, 1 \mathrm{H}), 2.37(\mathrm{~s}, 3 \mathrm{H}), 2.26(\mathrm{~s}, 12 \mathrm{H}) ;{ }^{13} \mathrm{C}$ NMR $(101 \mathrm{MHz}$, $\left.\mathrm{CDCl}_{3}\right) \delta: 158.9,147.5,145.8,140.6,137.6,137.0,129.1,125.4,119.9,82.0,25.4$, 21.5. HRMS (ESI) m/z: $[\mathrm{M}+\mathrm{H}]^{+}$calcd for $\mathrm{C}_{23} \mathrm{H}_{26} \mathrm{NO} 332.2009$; Found 332.2015.

\section{Bis(3,5-diisopropylphenyl)(2-methylpyridin-3-yl)methanol (S1c)}<smiles>Cc1ncccc1C(O)(c1cc(C(C)C)cc(C(C)C)c1)c1cc(C(C)C)cc(C(C)C)c1</smiles>

A colorless oil and solidified slowly by standing, $1.31 \mathrm{~g}, 59 \%$ yield. ${ }^{1} \mathrm{H}$ NMR (400 $\left.\mathrm{MHz}, \mathrm{CDCl}_{3}\right) \delta: 8.07-8.02(\mathrm{~m}, 1 \mathrm{H}), 7.22(\mathrm{dd}, J=8.0,1.6 \mathrm{~Hz}, 1 \mathrm{H}), 6.99(\mathrm{~s}, 2 \mathrm{H})$, 6.91 (dd, $J=8.0,4.8 \mathrm{~Hz}, 1 \mathrm{H}), 6.89$ (d, $J=1.6 \mathrm{~Hz}, 4 \mathrm{H}), 4.53$ (brs, 1H), 2.91-2.73 $(\mathrm{m}, 4 \mathrm{H}), 2.31(\mathrm{~s}, 3 \mathrm{H}), 1.15(\mathrm{~d}, J=2.0 \mathrm{~Hz}, 12 \mathrm{H}), 1.14(\mathrm{~d}, J=2.0 \mathrm{~Hz}, 12 \mathrm{H}) ;{ }^{13} \mathrm{C}$ NMR (101 MHz, $\left.\mathrm{CDCl}_{3}\right) \delta: 158.8,148.5,147.4,145.7,140.8,136.6,123.6,123.6$, 119.9, 82.4, 34.2, 25.2, 24.1, 24.0. HRMS (ESI) m/z: $[\mathrm{M}+\mathrm{H}]^{+}$calcd for $\mathrm{C}_{31} \mathrm{H}_{42} \mathrm{NO}$ 444.3261; Found 444.3265. 
Bis(3,5-di-tert-butylphenyl)(2-methylpyridin-3-yl)methanol (S1d)<smiles>CC(C)(C)c1cc(C(C)(C)C)cc(C(O)(c2cc(C(C)(C)C)cc(C(C)(C)C)c2)c2cccnc2N)c1</smiles>

White solid, m.p. $83-84{ }^{\circ} \mathrm{C}, 2.37 \mathrm{~g}, 95 \%$ yield. ${ }^{1} \mathrm{H}$ NMR $\left(400 \mathrm{MHz}, \mathrm{CDCl}_{3}\right) \delta: 8.19$ $(\mathrm{dd}, J=4.8,1.6 \mathrm{~Hz}, 1 \mathrm{H}), 7.31(\mathrm{t}, J=1.6 \mathrm{~Hz}, 2 \mathrm{H}), 7.25-7.21(\mathrm{~m}, 1 \mathrm{H}), 7.01(\mathrm{~d}, J=$ $1.6 \mathrm{~Hz}, 4 \mathrm{H}), 6.96(\mathrm{dd}, J=7.6,4.8 \mathrm{~Hz}, 1 \mathrm{H}), 3.64(\mathrm{~s}, 1 \mathrm{H}), 2.29(\mathrm{~s}, 3 \mathrm{H}), 1.21(\mathrm{~s}, 36 \mathrm{H})$; ${ }^{13} \mathrm{C}$ NMR (101 MHz, $\left.\mathrm{CDCl}_{3}\right) \delta: 158.8,150.2,147.2,145.1,141.1,136.5,122.4$, 120.8, 119.8, 82.8, 34.9, 31.5, 25.1. HRMS (ESI) m/z: $[\mathrm{M}+\mathrm{H}]^{+}$calcd for $\mathrm{C}_{35} \mathrm{H}_{50} \mathrm{NO}$ 500.3887; Found 500.3890.

\section{Synthesis of 3-diarylmethyl-2-methylpyridines $\mathbf{S 2}$.}

General procedure: To a solution of $\mathbf{S} 1(2.5 \mathrm{mmol})$ in dry $\mathrm{CH}_{2} \mathrm{Cl}_{2}(25 \mathrm{~mL})$ was added TFA $(1.9 \mathrm{~mL}, 25$ $\mathrm{mmol}), \mathrm{Et}_{3} \mathrm{SiH}(1.2 \mathrm{~mL}, 7.5 \mathrm{mmol})$, and $\mathrm{TfOH}(20 \mu \mathrm{L}, 0.25 \mathrm{mmol})$ sequentially via syringes under nitrogen atmosphere at $0{ }^{\circ} \mathrm{C}$. The resulting reaction mixture was stirred at room temperature for $6 \mathrm{~h}$ to complete the reaction, and quenched with water $(15 \mathrm{~mL})$. The layer was separated and the aqueous layer was extracted with dichloromethane $(3 \times 10 \mathrm{~mL})$. The combined organic layers were washed with aqueous $\mathrm{NaHCO}_{3}$ and brine, dried over $\mathrm{MgSO}_{4}$, and concentrated in vacuo. The residue was purified by flash chromatography with petroleum ether/ethyl acetate $(8: 1)$ as an eluent to yield the corresponding products $\mathbf{S 2}$ as white solids or colorless oils.

\section{3-Benzhydryl-2-methylpyridine (S2a)}<smiles>Cc1ncccc1C(c1ccccc1)c1ccccc1</smiles>

White solid, m.p. $78-79{ }^{\circ} \mathrm{C}, 0.48$ g, $74 \%$ yield. ${ }^{1} \mathrm{H}$ NMR $\left(400 \mathrm{MHz}, \mathrm{CDCl}_{3}\right) \delta: 8.37$ (d, $J=4.0 \mathrm{~Hz}, 1 \mathrm{H}), 7.28(\mathrm{t}, J=7.2 \mathrm{~Hz}, 4 \mathrm{H}), 7.21(\mathrm{t}, J=7.2 \mathrm{~Hz}, 2 \mathrm{H}), 7.10(\mathrm{~d}, J=7.2 \mathrm{~Hz}$, $1 \mathrm{H}), 7.06-6.99(\mathrm{~m}, 5 \mathrm{H}), 5.64(\mathrm{~s}, 1 \mathrm{H}), 2.45(\mathrm{~s}, 3 \mathrm{H}) ;{ }^{13} \mathrm{C} \mathrm{NMR}\left(101 \mathrm{MHz}, \mathrm{CDCl}_{3}\right) \delta$ : 157.3, 147.0, 142.3, 137.7, 136.8, 129.5, 128.6, 126.7, 121.1, 53.2, 22.8. HRMS (ESI) $\mathrm{m} / \mathrm{z}:[\mathrm{M}+\mathrm{H}]^{+}$calcd for $\mathrm{C}_{19} \mathrm{H}_{18} \mathrm{~N} 260.1434$; Found 260.1436 .

\section{3-(Bis(3,5-dimethylphenyl)methyl)-2-methylpyridine (S2b)}<smiles>Cc1cc(C)cc(C(c2cc(C)cc(C)c2)c2cccnc2C)c1</smiles>

White solid, m.p. $119-120{ }^{\circ} \mathrm{C}, 0.72$ g, $91 \%$ yield. ${ }^{1} \mathrm{H}$ NMR (400 $\left.\mathrm{MHz}, \mathrm{CDCl}_{3}\right) \delta$ : 8.37 (dd, $J=4.8,1.6 \mathrm{~Hz}, 1 \mathrm{H}), 7.12$ (dd, $J=7.6,1.6 \mathrm{~Hz}, 1 \mathrm{H}), 7.04$ (dd, $J=8.0,4.8$ $\mathrm{Hz}, 1 \mathrm{H}), 6.87$ (s, 2H), 6.65 (s, 4H), 5.49 (s, 1H), 2.45 (s, 3H), $2.25(\mathrm{~s}, 12 \mathrm{H}) ;{ }^{13} \mathrm{C}$ $\operatorname{NMR}\left(101 \mathrm{MHz}, \mathrm{CDCl}_{3}\right) \delta: 157.3,146.7,142.3,138.1,137.9,136.9,128.3,127.3$, 121.1, 53.0, 22.9, 21.4. HRMS (ESI) m/z: $[\mathrm{M}+\mathrm{H}]^{+}$calcd for $\mathrm{C}_{23} \mathrm{H}_{26} \mathrm{NO} 316.2060$; Found 316.2063.

\section{3-(Bis(3,5-diisopropylphenyl)methyl)-2-methylpyridine (S2c)}

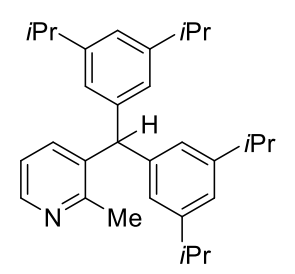

A colorless oil and solidified slowly by standing, $1.01 \mathrm{~g}, 95 \%$ yield. ${ }^{1} \mathrm{H}$ NMR (400 $\left.\mathrm{MHz} \mathrm{CDCl}_{3}\right) \delta: 8.36(\mathrm{~d}, J=3.6 \mathrm{~Hz}, 1 \mathrm{H}), 7.13(\mathrm{~d}, J=7.6 \mathrm{~Hz}, 1 \mathrm{H}), 7.05(\mathrm{dd}, J=$ 7.6, $4.8 \mathrm{~Hz}, 1 \mathrm{H}), 6.94(\mathrm{~s}, 2 \mathrm{H}), 6.70(\mathrm{~s}, 4 \mathrm{H}), 5.56(\mathrm{~s}, 1 \mathrm{H}), 2.90-2.75(\mathrm{~m}, 4 \mathrm{H}), 2.50$ $(\mathrm{s}, 3 \mathrm{H}), 1.18(\mathrm{~d}, J=6.8 \mathrm{~Hz}, 24 \mathrm{H}) ;{ }^{13} \mathrm{C} \mathrm{NMR}\left(101 \mathrm{MHz}, \mathrm{CDCl}_{3}\right) \delta: 157.3,148.8$, 146.6, 142.2, 138.7, 136.8, 125.2, 122.6, 121.0, 53.3, 34.1, 24.1, 2.0, 22.9. HRMS (ESI) $\mathrm{m} / \mathrm{z}:[\mathrm{M}+\mathrm{H}]^{+}$calcd for $\mathrm{C}_{31} \mathrm{H}_{42} \mathrm{~N} 428.3312$; Found 428.3315 . 


\section{3-(Bis(3,5-di-tert-butylphenyl)methyl)-2-methylpyridine (S2d)}<smiles>Cc1ncccc1C(c1cc(C(C)(C)C)cc(C(C)(C)C)c1)(c1cc(C(C)(C)C)cc(C(C)(C)C)c1)c1ccccn1</smiles>

White solid, m.p. $139-140{ }^{\circ} \mathrm{C}, 1.16 \mathrm{~g}, 96 \%$ yield. ${ }^{1} \mathrm{H}$ NMR (400 $\left.\mathrm{MHz}, \mathrm{CDCl}_{3}\right) \delta$ : $8.34(\mathrm{dd}, J=4.8,1.6 \mathrm{~Hz}, 1 \mathrm{H}), 7.25(\mathrm{~m}, \mathrm{~d}, J=1.6 \mathrm{~Hz}, 2 \mathrm{H}), 7.13(\mathrm{dd}, J=7.6,1.6$ $\mathrm{Hz}, 1 \mathrm{H}), 7.03(\mathrm{dd}, J=7.6,4.8 \mathrm{~Hz}, 1 \mathrm{H}), 6.86(\mathrm{~d}, J=1.6 \mathrm{~Hz}, 4 \mathrm{H}), 5.57(\mathrm{~s}, 1 \mathrm{H})$, $2.51(\mathrm{~s}, 3 \mathrm{H}), 1.23(\mathrm{~s}, 36 \mathrm{H}) ;{ }^{13} \mathrm{C}$ NMR $\left(101 \mathrm{MHz} \mathrm{CDCl}_{3}\right) \delta: 157.3,150.5,146.6$, 141.4, 139.2, 136.6, 123.9, 121.0, 120.0, 53.8, 34.8, 31.5, 23.0. HRMS (ESI) m/z: $[\mathrm{M}+\mathrm{H}]^{+}$calcd for $\mathrm{C}_{35} \mathrm{H}_{50} \mathrm{~N} 484.3938$; Found 484.3942 .

\section{Synthesis of 3-diarylmethyl picolinaldehydes S3.}

General procedure: The mixture of $\mathbf{S 2}(1.0 \mathrm{mmol})$ and $\mathrm{SeO}_{2}(666 \mathrm{mg}, 6.0 \mathrm{mmol})$ in dioxane $(4 \mathrm{~mL})$ was stirred at reflux in an oil bath under nitrogen atmosphere for $20 \mathrm{~h}$. The reaction mixture was cooled to room temperature, filtered, and concentrated in vacuo. The residue was purified by flash chromatography with petroleum ether/ethyl acetate $(5: 1)$ as an eluent to afford the desired picolinaldehyde $\mathbf{S 3}$ as white solids or colorless oils.

\section{3-Benzhydrylpicolinaldehyde (S3a)}<smiles>O=Cc1ncccc1C(c1ccccc1)(c1ccccc1)c1ccccc1</smiles>

White solid, m.p. $112-113{ }^{\circ} \mathrm{C}, 0.17 \mathrm{~g}, 64 \%$ yield. ${ }^{1} \mathrm{H}$ NMR (400 MHz, $\left.\mathrm{CDCl}_{3}\right) \delta: 10.09$ (s, 1H), 8.65 (dd, $J=4.4,1.6 \mathrm{~Hz}, 1 \mathrm{H}), 7.44$ (d, $J=7.6 \mathrm{~Hz}, 1 \mathrm{H}), 7.34$ (dd, $J=8.0,4.4$ $\mathrm{Hz}, 1 \mathrm{H}), 7.27-7.21(\mathrm{~m}, 4 \mathrm{H}), 7.21-7.15(\mathrm{~m}, 2 \mathrm{H}), 7.04-6.99(\mathrm{~m}, 4 \mathrm{H}), 6.81(\mathrm{~s}, 1 \mathrm{H}) ;{ }^{13} \mathrm{C}$ NMR (101 MHz, $\left.\mathrm{CDCl}_{3}\right)$ $\delta: 194.8,149.4,148.0,142.4,141.6,139.1,129.6,128.5$, 126.7, 126.6, 49.6. HRMS (ESI) m/z: $[\mathrm{M}+\mathrm{H}]^{+}$calcd for $\mathrm{C}_{19} \mathrm{H}_{16} \mathrm{NO} 274.1226$; Found 274.1230 .

\section{3-(Bis(3,5-dimethylphenyl)methyl)picolinaldehyde (S3b)}<smiles>Cc1cc(C)cc(C(c2cc(C)cc(C)c2)c2cccnc2C)c1</smiles>

White solid, m.p. $133-134{ }^{\circ} \mathrm{C}, 0.21 \mathrm{~g}, 63 \%$ yield. ${ }^{1} \mathrm{H}$ NMR $\left(400 \mathrm{MHz}, \mathrm{CDCl}_{3}\right) \delta$ : $10.23(\mathrm{~s}, 1 \mathrm{H}), 8.74(\mathrm{~d}, J=4.8 \mathrm{~Hz}, 1 \mathrm{H}), 7.61$ (d, $J=8.0 \mathrm{~Hz}, 1 \mathrm{H}), 7.47-7.38(\mathrm{~m}, 1 \mathrm{H})$, $6.93(\mathrm{~s}, 2 \mathrm{H}), 6.85-6.70(\mathrm{~m}, 5 \mathrm{H}), 2.31(\mathrm{~s}, 12 \mathrm{H}) ;{ }^{13} \mathrm{C} \mathrm{NMR}\left(101 \mathrm{MHz}, \mathrm{CDCl}_{3}\right) \delta$ : 194.7, 149.3, 147.8, 142.5, 142.0, 139.4, 137.9, 128.4, 127.6, 126.6, 49.4, 21.4. HRMS (ESI) m/z: $[\mathrm{M}+\mathrm{H}]^{+}$calcd for $\mathrm{C}_{23} \mathrm{H}_{24} \mathrm{NO} 330.1852$; Found 330.1855.

\section{3-(Bis(3,5-diisopropylphenyl)methyl)picolinaldehyde (S3c)}<smiles>Cc1ncccc1C(c1cc(C(C)C)cc(C(C)C)c1)(c1cc(C(C)C)cc(C(C)C)c1)c1cccnc1C</smiles>

A colorless oil and solidified slowly by standing, $0.20 \mathrm{~g}, 45 \%$ yield. ${ }^{1} \mathrm{H}$ NMR (400 $\left.\mathrm{MHz}, \mathrm{CDCl}_{3}\right) \delta: 10.19(\mathrm{~s}, 1 \mathrm{H}), 8.67(\mathrm{dd}, J=4.4,1.6 \mathrm{~Hz}, 1 \mathrm{H}), 7.57-7.46(\mathrm{~m}, 1 \mathrm{H})$, 7.44-7.33 (m, 1H), 7.00-6.88 (m, 2H), 6.82-6.76 (m, 1H), 6.74-6.64 (m, 4H), 2.87-2.72 (m, 4H), $1.17(\mathrm{~d}, J=10.8 \mathrm{~Hz}, 24 \mathrm{H}) ;{ }^{13} \mathrm{C} \mathrm{NMR}\left(101 \mathrm{MHz}, \mathrm{CDCl}_{3}\right) \delta$ : 194.6, 149.4, 148.7, 147.7, 142.8, 142.3, 139.2, 126.5, 125.4, 122.7, 49.4, 34.1, 24.1, 24.0. HRMS (ESI) m/z: [M+Na $]^{+}$calcd for $\mathrm{C}_{31} \mathrm{H}_{39} \mathrm{NONa} 464.2924$; Found 464.2928. 
<smiles>Cc1ncccc1C(c1cc(C(C)(C)C)cc(C(C)(C)C)c1)c1cc(C(C)(C)C)cc(C(C)(C)C)c1</smiles>

White solid, m.p. $154-155{ }^{\circ} \mathrm{C}, 0.29$ g, $58 \%$ yield. ${ }^{1} \mathrm{H}$ NMR $\left(400 \mathrm{MHz}, \mathrm{CDCl}_{3}\right) \delta$ : $10.20(\mathrm{~s}, 1 \mathrm{H}), 8.68(\mathrm{dd}, J=4.4,1.6 \mathrm{~Hz}, 1 \mathrm{H}), 7.54$ (d, $J=7.6 \mathrm{~Hz}, 1 \mathrm{H}), 7.39$ (dd, $J=$ 8.0, 4.4 Hz, 1H), 7.29-7.23 (m, 2H), 6.94-6.89 (m, 4H), $6.82(\mathrm{~s}, 1 \mathrm{H}), 1.23(\mathrm{~s}, 36 \mathrm{H})$; ${ }^{13} \mathrm{C}$ NMR (101 MHz, $\left.\mathrm{CDCl}_{3}\right) \delta: 194.7,150.5,149.4,147.7,143.3,141.4,139.0$, 126.3, 124.1, 120.2, 49.9, 34.8, 31.4. HRMS (ESI) m/z: $[\mathrm{M}+\mathrm{Na}]^{+}$calcd for $\mathrm{C}_{35} \mathrm{H}_{47} \mathrm{NONa} 520.3550$; Found 520.3555.

\section{Preparation of new SpiroPAP ligands}

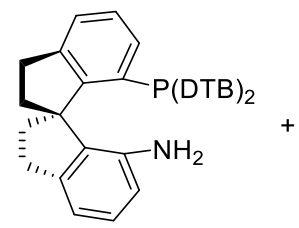

(R)-DTB-SpiroAP<smiles>NC(=O)c1cccnc1C=O</smiles>

S3

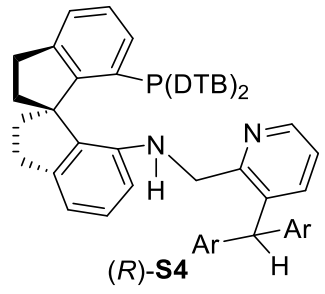

(R)-S4

General procedure: ${ }^{1}$ The mixture of $(R)$-DTB-SpiroAP $(283 \mathrm{mg}, 0.44 \mathrm{mmol})$ and picolinaldehyde $\mathbf{S 3}$ $(0.88 \mathrm{mmol})$ in DCE $(15 \mathrm{~mL})$ was stirred at $45^{\circ} \mathrm{C}$ in an oil bath under nitrogen atmosphere for $20-30 \mathrm{~h}$ (imine intermediate was no longer increased). $\mathrm{NaBH}(\mathrm{OAc})_{3}(373 \mathrm{mg}, 1.76 \mathrm{mmol})$ was then added and the resulting reaction mixture was stirred at the same temperature for $12 \mathrm{~h}$. The reaction mixture was then quenched with aqueous saturated $\mathrm{NaHCO}_{3}$ solution $(15 \mathrm{~mL})$, extracted with ethyl acetate $(3 \times 10$ $\mathrm{mL}$ ). The extracts were dried with anhydrous $\mathrm{Na}_{2} \mathrm{SO}_{4}$, concentrated in vacuo, and purified by chromatography on silica gel column with petroleum ether/ethyl acetate (20:1) as eluent to afford the corresponding ligand $(R)$-S4 as white solids.

\section{Ligand $(R)-S 4 a$}

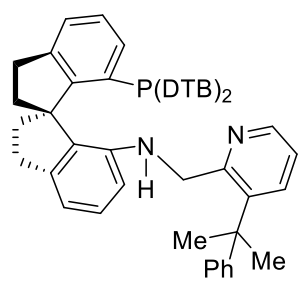

White solid, m.p. $74-75^{\circ} \mathrm{C}, 0.35$ g, $94 \%$ yield. $[\alpha]_{\mathrm{D}}^{27}+107.2\left(c 1.0, \mathrm{CHCl}_{3}\right) .{ }^{1} \mathrm{H}$ NMR (400 MHz, $\left.\mathrm{CDCl}_{3}\right) \delta: 7.83(\mathrm{dd}, J=4.6,1.4 \mathrm{~Hz}, 1 \mathrm{H}), 7.75(\mathrm{dd}, J=8.0,1.4$ $\mathrm{Hz}, 1 \mathrm{H}), 7.2-7.21$ (m, 5H), 7.18-7.07 (m, 5H), 7.07-6.99 (m, 2H), 6.83 (dd, $J=$ 7.9, $1.8 \mathrm{~Hz}, 2 \mathrm{H}), 6.68(\mathrm{dd}, J=7.4,1.8 \mathrm{~Hz}, 2 \mathrm{H}), 6.55(\mathrm{~d}, J=7.4 \mathrm{~Hz}, 1 \mathrm{H}), 5.74$ $(\mathrm{d}, J=7.8 \mathrm{~Hz}, 1 \mathrm{H}), 5.24(\mathrm{~d}, J=7.4 \mathrm{~Hz}, 1 \mathrm{H}), 3.81-3.58(\mathrm{~m}, 2 \mathrm{H}), 2.97-2.86(\mathrm{~m}$, $2 \mathrm{H}), 2.84-2.74(\mathrm{~m}, 1 \mathrm{H}), 2.56-2.50(\mathrm{~m}, 1 \mathrm{H}), 2.36-2.28(\mathrm{~m}, 1 \mathrm{H}), 1.96-1.88(\mathrm{~m}$, 2H), 1.72-1.66 (m, 1H), $1.60(\mathrm{~s}, 6 \mathrm{H}), 1.16(\mathrm{~s}, 18 \mathrm{H}), 0.97(\mathrm{~s}, 18 \mathrm{H}) ;{ }^{31} \mathrm{P} \mathrm{NMR}\left(162 \mathrm{MHz}, \mathrm{CDCl}_{3}\right) \delta:-16.26$ (s); ${ }^{13} \mathrm{C}$ NMR (101 MHz, $\left.\mathrm{CDCl}_{3}\right) \delta: 156.0,152.1,151.9,149.9,149.8,149.4,149.3,149.2,146.0$, 145.2(2), 144.2(2), 144.0(2), 141.6, 137.5, 137.4, 137.2, 137.1, 135.2, 135.0, 133.2, 133.0, 131.7(2), 129.0, 128.8, 128.6, 128.3, 128.0, 127.8, 126.3, 126.2, 125.7, 125.2, 121.7, 121.1, 120.9, 112.7, 107.4, 71.4, 61.6, 46.8, 42.6, 42.3, 37.6, 35.6, 34.8, 34.6, 31.4, 31.3(2), 31.1, 30.8, 30.6(2), 27.0. HRMS (ESI) $\mathrm{m} / \mathrm{z}:[\mathrm{M}+\mathrm{H}]^{+}$calcd for $\mathrm{C}_{60} \mathrm{H}_{74} \mathrm{~N}_{2} \mathrm{P} 853.5584$; Found 853.5588 .

\section{Ligand ( $R$ )-S4b}

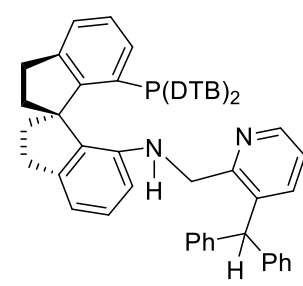

White solid, m.p. $86-87^{\circ} \mathrm{C}, 0.33$ g, $83 \%$ yield. $[\alpha]_{\mathrm{D}}^{27}+164.6\left(c 1.0, \mathrm{CHCl}_{3}\right) .{ }^{1} \mathrm{H}$ NMR (400 MHz, $\left.\mathrm{CDCl}_{3}\right) \delta: 7.85(\mathrm{~d}, J=4.8 \mathrm{~Hz}, 1 \mathrm{H}), 7.27-7.22(\mathrm{~m}, 2 \mathrm{H}), 7.19$ $7.10(\mathrm{~m}, 7 \mathrm{H}), 7.08-7.01$ (m, 3H), 6.98-6.92 (m, 3H), 6.90-6.84 (m, 3H), 6.78 (dd, $J=7.8,1.6 \mathrm{~Hz}, 2 \mathrm{H}), 6.62(\mathrm{dd}, J=7.8,1.6 \mathrm{~Hz}, 2 \mathrm{H}), 6.54(\mathrm{~d}, J=8.0 \mathrm{~Hz}, 1 \mathrm{H})$, $6.15(\mathrm{~d}, J=8.0 \mathrm{~Hz}, 1 \mathrm{H}), 5.51$ (s, $1 \mathrm{H}), 5.25$ (d, $J=7.6 \mathrm{~Hz}, 1 \mathrm{H}), 4.00$ (dd, $J=15.6$, 
$6.0 \mathrm{~Hz}, 1 \mathrm{H}), 3.66(\mathrm{~d}, J=15.2 \mathrm{~Hz}, 1 \mathrm{H}), 3.00-2.85(\mathrm{~m}, 2 \mathrm{H}), 2.84-2.74(\mathrm{~m}, 1 \mathrm{H}), 2.58-2.52(\mathrm{~m}, 1 \mathrm{H}), 2.41-$ $2.27(\mathrm{~m}, 1 \mathrm{H}), 1.98-1.93(\mathrm{~m}, 2 \mathrm{H}), 1.85-1.72(\mathrm{~m}, 1 \mathrm{H}), 1.10(\mathrm{~s}, 18 \mathrm{H}), 0.90(\mathrm{~s}, 18 \mathrm{H}) ;{ }^{31} \mathrm{P}$ NMR $(162 \mathrm{MHz}$, $\left.\mathrm{CDCl}_{3}\right) \delta:-16.33(\mathrm{~s}) ;{ }^{13} \mathrm{C} \mathrm{NMR}\left(101 \mathrm{MHz}, \mathrm{CDCl}_{3}\right) \delta: 155.1,152.2,151.9,149.9(2), 149.5,149.4,146.2$, 144.7(2), 144.3, 144.2(2), 142.0, 141.7, 137.6, 137.5, 137.1, 137.0, 136.5, 135.1, 134.9, 133.1, 132.2(2), 129.4, 129.2, 128.8, 128.6, 128.3, 127.9, 127.7, 126.8(2), 126.5, 125.3, 121.7, 121.2, 121.1, 113.2, 107.7, 100.0, 71.4, 61.7, 51.7, 45.6, 42.6, 38.0, 36.1, 34.8, 34.6, 31.4, 31.3, 31.2, 30.8. HRMS (ESI) m/z: $[\mathrm{M}+\mathrm{H}]^{+}$calcd for $\mathrm{C}_{64} \mathrm{H}_{74} \mathrm{~N}_{2} \mathrm{P} 901.5584$; Found 901.5588 .

\section{Ligand $(R)$-S4c}

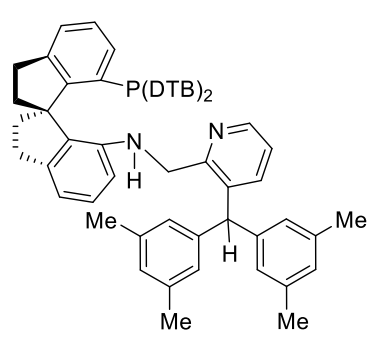

White solid, m.p. $91-92{ }^{\circ} \mathrm{C}, 0.39 \mathrm{~g}, 93 \%$ yield. $[\alpha]_{\mathrm{D}}^{27}+151.0\left(c 1.0, \mathrm{CHCl}_{3}\right)$. ${ }^{1} \mathrm{H}$ NMR (400 MHz, $\left.\mathrm{CDCl}_{3}\right) \delta: 8.05(\mathrm{dd}, J=4.7,1.4 \mathrm{~Hz}, 1 \mathrm{H}), 7.45-7.43$ (m, 1H), 7.36-7.32 (m, 2H), 7.29-7.23 (m, 2H), 7.23-7.16 (m, 2H), 7.06$7.02(\mathrm{~m}, 1 \mathrm{H}), 6.97(\mathrm{dd}, J=7.8,1.7 \mathrm{~Hz}, 3 \mathrm{H}), 6.91(\mathrm{~s}, 1 \mathrm{H}), 6.83(\mathrm{dd}, J=7.5$, $1.7 \mathrm{~Hz}, 2 \mathrm{H}), 6.74-6.72(\mathrm{~m}, 3 \mathrm{H}), 6.66(\mathrm{~s}, 2 \mathrm{H}), 6.39$ (d, $J=7.9 \mathrm{~Hz}, 1 \mathrm{H}), 5.52$ (s, 1H), 5.43-5.33 (m, 1H), $4.11(\mathrm{dd}, J=16.0,5.6 \mathrm{~Hz}, 1 \mathrm{H}), 3.92(\mathrm{~d}, J=14.6$ $\mathrm{Hz}, 1 \mathrm{H}), 3.20-3.05(\mathrm{~m}, 2 \mathrm{H}), 3.02-2.94(\mathrm{~m}, 1 \mathrm{H}), 2.80-2.74(\mathrm{~m}, 1 \mathrm{H}), 2.58-$ $2.46(\mathrm{~m}, 1 \mathrm{H}), 2.35(\mathrm{~s}, 6 \mathrm{H}), 2.31(\mathrm{~s}, 6 \mathrm{H}), 2.19-2.12(\mathrm{~m}, 2 \mathrm{H}), 2.06-1.98(\mathrm{~m}, 1 \mathrm{H}), 1.29(\mathrm{~s}, 18 \mathrm{H}), 1.09(\mathrm{~s}$, 18H); ${ }^{31} \mathrm{P}$ NMR (162 MHz, $\left.\mathrm{CDCl}_{3}\right) \delta:-16.50(\mathrm{~s}) ;{ }^{13} \mathrm{C} \mathrm{NMR}\left(101 \mathrm{MHz}, \mathrm{CDCl}_{3}\right) \delta: 155.1,152.3,152.0$, $150.0,149.9,149.5,149.4,146.0,144.7(2), 144.3,144.2,142.1,141.6,137.9,137.8,137.7,137.6,137.2$, 137.0(2), 136.6, 135.1, 134.9, 133.2, 132.2, 132.2, 128.8, 128.6, 128.5, 128.4, 128.3, 127.9, 127.7, 127.4, 127.0, 126.6, 125.3, 121.7, 121.2, 121.1, 113.1, 107.9, 71.4, 61.7, 51.6, 45.8, 42.6, 38.1, 36.1, 34.8, 34.6, 31.4(2), 31.3, 31.2, 30.8, 30.3. HRMS (ESI) m/z: $[\mathrm{M}+\mathrm{H}]^{+}$calcd for $\mathrm{C}_{68} \mathrm{H}_{82} \mathrm{~N}_{2} \mathrm{P}$ 957.6210; Found 957.6212 .

\section{Ligand (R)-S4d}

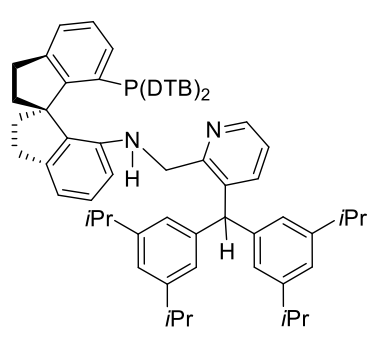

A colorless oil and solidified slowly by standing, $0.42 \mathrm{~g}, 90 \%$ yield. $[\alpha]_{\mathrm{D}}^{21}$ $+140.8\left(c 0.5, \mathrm{CHCl}_{3}\right) .{ }^{1} \mathrm{H}$ NMR $\left(400 \mathrm{MHz}, \mathrm{CDCl}_{3}\right) \delta: 7.86(\mathrm{~d}, J=4.4 \mathrm{~Hz}$, 1H), 7.26-7.24 (m, 1H), 7.17-7.12(m, 2H), 7.06 (s, 1H), 7.04-6.95 (m, 3H), 6.86-7.84 (m, 2H), 6.80-7.78 (m, 3H), 6.70-6.63 (m, 2H), $6.63(\mathrm{~s}, 2 \mathrm{H}), 6.57$ (s, 2H), 6.54-6.50 (m, 1H), $6.15(\mathrm{~d}, J=7.9 \mathrm{~Hz}, 1 \mathrm{H}), 5.34$ (s, 1H), 5.24-5.20 (m, 1H), 3.91 (dd, $J=16.0,5.4 \mathrm{~Hz}, 1 \mathrm{H}), 3.76(\mathrm{~d}, J=15.8 \mathrm{~Hz}, 1 \mathrm{H}), 3.00-2.87$ (m, 2H), 2.84-2.59 (m, 6H), 2.37-2.29 (m, 1H), 2.01-1.92 (m, 2H), 1.90$1.84(\mathrm{~m}, 1 \mathrm{H}), 1.12(\mathrm{~s}, 6 \mathrm{H}), 1.10(\mathrm{~s}, 18 \mathrm{H}), 1.06(\mathrm{~d}, J=6.7 \mathrm{~Hz}, 18 \mathrm{H}), 0.91$ (s, 18H); ${ }^{31} \mathrm{P}$ NMR (162 MHz, $\left.\mathrm{CDCl}_{3}\right) \delta:-16.73(\mathrm{~s}) ;{ }^{13} \mathrm{C} \mathrm{NMR}\left(101 \mathrm{MHz}, \mathrm{CDCl}_{3}\right) \delta: 154.1,151.3,151.1,148.8(2), 148.4,148.3,147.8$, 147.7, 144.7, 143.6, 143.5, 143.1(2), 143.07, 140.8, 140.4, 136.7, 136.6, 136.3, 136.2, 136.1, 135.1, 134.0, 133.8, 132.2(2), 131.3, 131.2, 127.7, 127.5, 127.2, 126.8, 126.6, 125.5, 124.2(2), 123.9, 121.6(2), 120.6, 120.0(2), 111.8, 106.8, 98.9, 70.3, 60.6, 51.2, 44.7, 41.5, 37.3, 35.1, 33.7, 33.5, 33.0(2), 30.6, 30.3, 30.2(2), 29.8, 23.2, 23.1, 22.9, 22.8, 21.6, 13.1. HRMS (ESI) m/z: $[\mathrm{M}+\mathrm{H}]^{+}$calcd for $\mathrm{C}_{76} \mathrm{H}_{98} \mathrm{~N}_{2} \mathrm{P}$ 1069.7462; Found 1069.7468. 


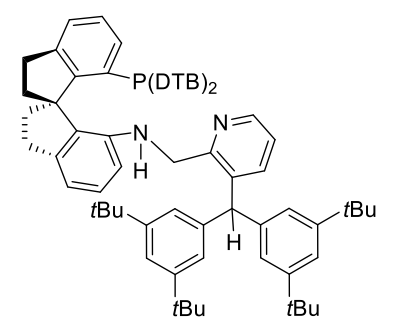

A colorless oil and solidified slowly by standing, $0.42 \mathrm{~g}, 85 \%$ yield. $[\alpha]_{\mathrm{D}}^{20}$ $+130.8\left(c 1.0, \mathrm{CHCl}_{3}\right) .{ }^{1} \mathrm{H}$ NMR $\left(400 \mathrm{MHz}, \mathrm{CDCl}_{3}\right)$ ): $7.95(\mathrm{dd}, J=4.7,1.4$ $\mathrm{Hz}, 1 \mathrm{H}), 7.34-7.32(\mathrm{~m}, 1 \mathrm{H}), 7.28-7.26(\mathrm{~m}, 1 \mathrm{H}), 7.25-7.23(\mathrm{~m}, 1 \mathrm{H}), 7.22-$ $7.18(\mathrm{~m}, 2 \mathrm{H}), 7.14-7.04(\mathrm{~m}, 4 \mathrm{H}), 6.96-6.92(\mathrm{~m}, 1 \mathrm{H}), 6.89-6.88(\mathrm{~m}, 3 \mathrm{H})$, $6.87(\mathrm{~d}, J=1.8 \mathrm{~Hz}, 1 \mathrm{H}), 6.83(\mathrm{~d}, J=1.6 \mathrm{~Hz}, 2 \mathrm{H}), 6.76(\mathrm{dd}, J=7.5,1.7 \mathrm{~Hz}$, $2 \mathrm{H}), 6.60(\mathrm{~d}, J=7.4 \mathrm{~Hz}, 1 \mathrm{H}), 6.17(\mathrm{~d}, J=7.9 \mathrm{~Hz}, 1 \mathrm{H}), 5.40(\mathrm{~s}, 1 \mathrm{H}), 5.35-$ $5.33(\mathrm{~m}, 1 \mathrm{H}), 3.97(\mathrm{dd}, J=16.1,5.2 \mathrm{~Hz}, 1 \mathrm{H}), 3.88(\mathrm{dd}, J=16.1,2.4 \mathrm{~Hz}$, $1 \mathrm{H}), 3.08-2.96(\mathrm{~m}, 2 \mathrm{H}), 2.92-2.84(\mathrm{~m}, 1 \mathrm{H}), 2.77-2.71(\mathrm{~m}, 1 \mathrm{H}), 2.42-2.32(\mathrm{~m}, 1 \mathrm{H}), 2.12-1.98(\mathrm{~m}, 3 \mathrm{H})$, 1.24 (s, 18H), 1.21 (s, 18H), 1.17 (s, 18H), 0.99 (s, 18H); ${ }^{31} \mathrm{P}$ NMR (162 MHz, $\left.\mathrm{CDCl}_{3}\right) \delta:-17.09(\mathrm{~s}) ;{ }^{13} \mathrm{C}$ NMR (101 MHz, $\left.\mathrm{CDCl}_{3}\right) \delta: 155.2,152.6,152.3,150.6(2), 149.9,149.8,149.4(2), 145.8,144.6(2)$, 144.2(2), 144.1(2), 140.8, 137.9, 137.8, 137.6, 137.4, 137.3, 135.9, 135.0, 134.8, 133.4, 132.6, 132.5, 128.7, 128.5, 128.3, 127.8, 127.6, 126.6, 125.2, 124.0, 123.6(2), 121.0, 120.4, 112.7, 108.0, 71.4, 61.7, 53.0, 45.8, 42.6, 38.7, 36.2, 34.9, 34.8, 34.6, 31.6, 31.5, 31.4, 31.3, 30.9, 30.3, 30.2, 29.8. HRMS (ESI) $\mathrm{m} / \mathrm{z}:[\mathrm{M}+\mathrm{H}]^{+}$calcd for $\mathrm{C}_{80} \mathrm{H}_{106} \mathrm{~N}_{2} \mathrm{P} 1125.8088$; Found 1125.8090 .

\section{Preparation of chiral spiro iridium catalysts}

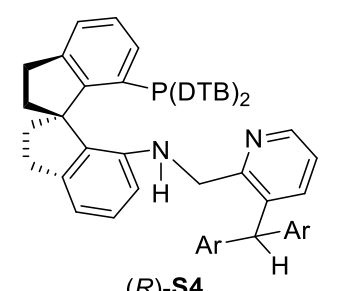

(R)-S4

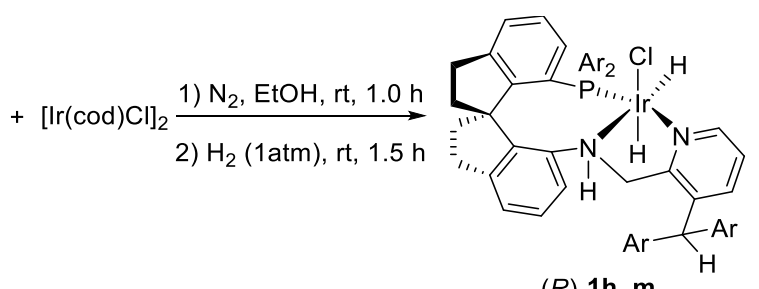

$(R)-1 \mathrm{~h}-\mathrm{m}$

General procedure: ${ }^{1}$ To a mixture of $(R)-\mathbf{S 4}(6.3 \mu \mathrm{mol})$ and $[\operatorname{Ir}(\mathrm{COD}) \mathrm{Cl}]_{2}(2.0 \mathrm{mg}, 3.0 \mu \mathrm{mol})$ was added anhydrous EtOH $(2.0 \mathrm{~mL})$ in a dry Schlenk tube $(25 \mathrm{~mL})$ under $\mathrm{N}_{2}$ atmosphere. After stirring at room temperature for $1.0 \mathrm{~h}$, the Schlenk tube was purged and replaced with $\mathrm{H}_{2}$ three times. The reaction mixture was stirred at room temperature for $1.5 \mathrm{~h}$, then concentrated and dried in vacuum for $10 \mathrm{~h}$ to yield the corresponding product. The obtained chiral spiro iridium catalysts $(R)-\mathbf{1 h}-\mathbf{m}$ were used directly without further purification, or/and usually stored under inert atmosphere at room temperature.

\section{(B) Preparation of $\beta$-Aryl Alkylidene Malonate Esters}

$\beta$-Aryl substituted $\alpha, \beta$-unsaturated malonates were synthesized by palladium catalyzed coupling of $p$ toluenesulfonyloxyalkylene malonates $\mathbf{S 6}$ with arylboronic acids ${ }^{2}$ (Method A) or the indium mediated Knoevenagel condensation of malonates to terminal alkynes ${ }^{3}$ (Method B).

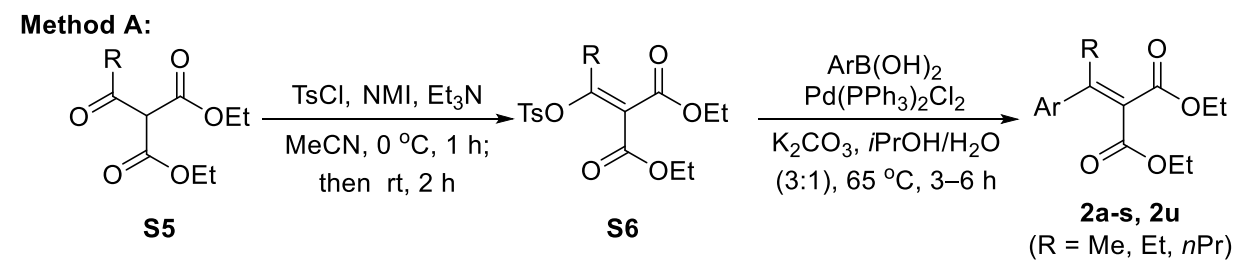

Method B:

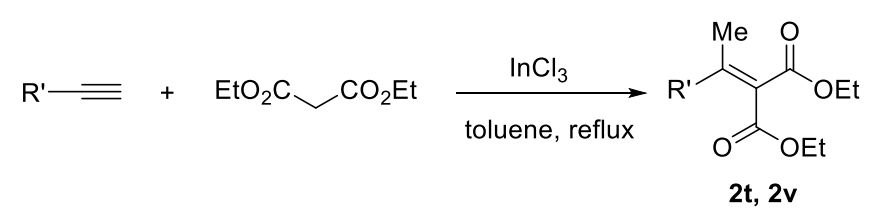


Method A: The $\beta$-aryl substituted $\alpha, \beta$-unsaturated malonates $\mathbf{2 a - s ,} \mathbf{2} \mathbf{u}$ were prepared by palladium catalyzed coupling of $p$-toluenesulfonyloxyalkylene malonates $\mathbf{S 6}$ with arylboronic acids according to literature method. ${ }^{2}$

\section{Synthesis of $p$-toluenesulfonyloxyalkylene malonates S6}

General procedure $^{2}$ : To a solution of $\mathbf{S 5}(10 \mathrm{mmol}), N$-methylimidazole (NMI) $(1.23 \mathrm{~g}, 15 \mathrm{mmol})$ and $\mathrm{Et}_{3} \mathrm{~N}(1.52 \mathrm{~g}, 15 \mathrm{mmol})$ in $\mathrm{MeCN}(10 \mathrm{~mL})$ was added a solution of $p$-toluenesulfonylchloride $(2.86 \mathrm{~g}, 15$ $\mathrm{mmol})$ in $\mathrm{MeCN}(10 \mathrm{~mL})$ at $0-5{ }^{\circ} \mathrm{C}$ under an argon atmosphere. The resulting mixture was stirred at the same temperature for $1 \mathrm{~h}$ and then at $20-25^{\circ} \mathrm{C}$ for $2 \mathrm{~h}$ to complete the reaction. The reaction mixture was diluted with water $(15 \mathrm{~mL})$ and extracted with $\mathrm{CH}_{2} \mathrm{Cl}_{2}(3 \times 10 \mathrm{~mL})$. The organic layer was dried with anhydrous $\mathrm{Na}_{2} \mathrm{SO}_{4}$ and concentrated in vacuo to afford the crude product. After chromatography on silica gel column with petroleum ether/ethyl acetate (10:1) as an eluent, the corresponding enol tosylate was obtained as a colorless oil. (Note: diethyl 2-acylmalonates $\mathbf{S 5}$ are known compounds ${ }^{4,5}$ and were synthesized according to the known procedure from diethyl malonate and acyl chloride).

\section{Diethyl-2-(1-(tosyloxy)ethylidene)malonate (S6a)}

$$
\begin{array}{ll}
\text { Colorless oil, } 2.24 \mathrm{~g}, 63 \% \text { yield. }{ }^{1} \mathrm{H} \text { NMR }\left(400 \mathrm{MHz}, \mathrm{CDCl}_{3}\right) \delta: 7.83(\mathrm{~d}, J=8.4 \mathrm{~Hz}, \\
\mathrm{CO}_{2} \mathrm{Et} & \begin{array}{l}
2 \mathrm{H}), 7.39(\mathrm{~d}, J=8.4 \mathrm{~Hz}, 2 \mathrm{H}), 4.21(\mathrm{q}, J=7.2 \mathrm{~Hz}, 2 \mathrm{H}), 4.10(\mathrm{q}, J=7.2 \mathrm{~Hz}, 2 \mathrm{H}), 2.45 \\
\delta: 162.9,162.8,159.9,145.9,133.2,129.9,129.0,119.8,61.4,21.5,18.5,13.8,13.6 .
\end{array}
\end{array}
$$

HRMS (ESI) m/z: [M+Na $]^{+}$calcd for $\mathrm{C}_{16} \mathrm{H}_{20} \mathrm{O}_{7} \mathrm{SNa} 379.0822$; Found 379.0826.

\section{Diethyl-2-(1-(tosyloxy)propylidene)malonate (S6b)}<smiles>CCOC(=O)C(OCC)=C([O+])CC</smiles>

Colorless oil, $3.37 \mathrm{~g}, 91 \%$ yield. ${ }^{1} \mathrm{H}$ NMR (400 MHz, $\left.\mathrm{CDCl}_{3}\right) \delta: 7.85$ (d, $J=8.4 \mathrm{~Hz}$, $2 \mathrm{H}), 7.38(\mathrm{~d}, J=8.4 \mathrm{~Hz}, 2 \mathrm{H}), 4.23(\mathrm{q}, J=7.2 \mathrm{~Hz}, 2 \mathrm{H}), 4.09(\mathrm{q}, J=7.2 \mathrm{~Hz}, 2 \mathrm{H}), 2.86$ $(\mathrm{q}, J=7.2 \mathrm{~Hz}, 2 \mathrm{H}), 2.46(\mathrm{~s}, 3 \mathrm{H}), 1.27(\mathrm{t}, J=7.2 \mathrm{~Hz}, 3 \mathrm{H}), 1.20(\mathrm{t}, J=7.2 \mathrm{~Hz}, 3 \mathrm{H})$, $1.13(\mathrm{t}, J=7.5 \mathrm{~Hz}, 3 \mathrm{H}) ;{ }^{13} \mathrm{C} \mathrm{NMR}\left(101 \mathrm{MHz}, \mathrm{CDCl}_{3}\right) \delta: 164.4,163.0,162.9,145.8$, 133.4, 129.8, 128.1 119.8, 61.6, 61.5, 25.4, 21.6, 13.8, 13.7, 11.2. HRMS (ESI) m/z: [M+Na $]^{+}$calcd for $\mathrm{C}_{17} \mathrm{H}_{22} \mathrm{O}_{7} \mathrm{SNa} 393.0978$; Found 393.0983.

\section{Diethyl-2-(1-(tosyloxy)butylidene)malonate (S6c)}<smiles>CCOC(=O)C(C(=O)OCC)=C([O-])C(C)C</smiles>

Colorless oil, $2.69 \mathrm{~g}, 70 \%$ yield. ${ }^{1} \mathrm{H}$ NMR (400 MHz, $\left.\mathrm{CDCl}_{3}\right) \delta: 7.85$ (d, J=8.0 Hz, $2 \mathrm{H}), 7.37(\mathrm{~d}, J=8.0 \mathrm{~Hz}, 2 \mathrm{H}), 4.22(\mathrm{q}, J=7.2 \mathrm{~Hz}, 2 \mathrm{H}), 4.10(\mathrm{q}, J=7.2 \mathrm{~Hz}, 2 \mathrm{H})$, 2.86-2.73 (m, 2H), $2.47(\mathrm{~s}, 3 \mathrm{H}), 1.64-1.50(\mathrm{~m}, 2 \mathrm{H}), 1.27(\mathrm{t}, J=7.2 \mathrm{~Hz}, 3 \mathrm{H}), 1.21(\mathrm{t}$, $J=7.2 \mathrm{~Hz}, 3 \mathrm{H}), 0.88(\mathrm{t}, J=7.6 \mathrm{~Hz}, 3 \mathrm{H}) ;{ }^{13} \mathrm{C} \mathrm{NMR}\left(101 \mathrm{MHz}, \mathrm{CDCl}_{3}\right) \delta: 163.3$, 163.2, 163.0, 145.7, 133.5, 129.8, 128.2, 120.5, 61.6, 33.5, 21.7, 20.3, 13.9, 13.8, 13.5. HRMS (ESI) m/z: $[\mathrm{M}+\mathrm{Na}]^{+}$calcd for $\mathrm{C}_{18} \mathrm{H}_{24} \mathrm{O}_{7} \mathrm{SNa} 407.1135$; Found 407.1140.

\section{Synthesis of $\beta$-aryl substituted $\alpha, \beta$-unsaturated malonates $2 \mathrm{a}-\mathrm{s}, 2 \mathrm{u}$}

General procedure $^{2}$ : A suspension of enol tosylate $\mathbf{S 6}$ (4 mmol), $\mathrm{ArB}(\mathrm{OH})_{2}(4.4 \mathrm{mmol}), \mathrm{K}_{2} \mathrm{CO}_{3}(1.66$ g, $12 \mathrm{mmol})$, and $\mathrm{Pd}\left(\mathrm{PPh}_{3}\right)_{2} \mathrm{Cl}_{2}(140 \mathrm{mg}, 0.2 \mathrm{mmol})$ in a mixture solvent of $i \mathrm{PrOH}(12 \mathrm{~mL})$ and water $(4 \mathrm{~mL})$ was stirred at $65^{\circ} \mathrm{C}$ in an oil bath under an argon atmosphere for $3-6 \mathrm{~h}$. The reaction mixture was cooled to room temperature, diluted with water $(15 \mathrm{~mL})$, and extracted with $\mathrm{CH}_{2} \mathrm{Cl}_{2}(3 \times 10 \mathrm{~mL})$. The combined organic layers were washed with brine, dried with anhydrous $\mathrm{Na}_{2} \mathrm{SO}_{4}$, and concentrated in 
vacuo. The crude product was purified by flash chromatography on a silica-gel with petroleum ether/ethyl acetate (10:1) as an eluent to give the corresponding desired product as a colorless oil.

Diethyl-2-(1-phenylethylidene)malonate (2a) ${ }^{3}$<smiles>CCOC(=O)C(C(=O)OCC)=C(c1ccccc1)[N+](=O)[O-]</smiles>

Colorless oil, 6 h, $0.57 \mathrm{~g}, 54 \%$ yield. ${ }^{1} \mathrm{H}$ NMR (400 MHz, $\left.\mathrm{CDCl}_{3}\right)$ 8: 7.38-7.29 (m, $3 \mathrm{H}), 7.26-7.16(\mathrm{~m}, 2 \mathrm{H}), 4.28$ (q, $J=7.2 \mathrm{~Hz}, 2 \mathrm{H}), 3.96$ (q, $J=7.2 \mathrm{~Hz}, 2 \mathrm{H}), 2.44$ (s,

$3 \mathrm{H}), 1.32(\mathrm{t}, J=7.2 \mathrm{~Hz}, 3 \mathrm{H}), 0.95(\mathrm{t}, J=7.2 \mathrm{~Hz}, 3 \mathrm{H})$ (the spectra are in accordance with those of the compound reported in the literature).

\section{Diethyl-2-(1-phenylpropylidene)malonate (2b) ${ }^{6}$}<smiles>CCOC(=O)C(C(=O)OCC)=C(CC)c1ccccc1</smiles>

Colorless oil, 4 h, 0.96 g, 87\% yield. ${ }^{1} \mathrm{H}$ NMR (400 MHz, $\left.\mathrm{CDCl}_{3}\right)$ 8: 7.39-7.30 (m, $3 \mathrm{H}), 7.24-7.11(\mathrm{~m}, 2 \mathrm{H}), 4.29(\mathrm{q}, J=7.2 \mathrm{~Hz}, 2 \mathrm{H}), 3.94(\mathrm{q}, J=7.2 \mathrm{~Hz}, 2 \mathrm{H}), 2.78$ (q, $J=7.6 \mathrm{~Hz}, 2 \mathrm{H}), 1.32(\mathrm{t}, J=7.2 \mathrm{~Hz}, 3 \mathrm{H}), 1.02(\mathrm{t}, J=7.6 \mathrm{~Hz}, 3 \mathrm{H}), 0.93(\mathrm{t}, J=7.2 \mathrm{~Hz}$, $3 \mathrm{H}$ ) (the spectra are in accordance with those of the compound reported in the literature).

\section{Diethyl-2-(1-phenylbutylidene)malonate (2c)}<smiles>CCOC(=O)C(C(=O)OCC)=C(c1ccccc1)c1ccccc1</smiles>

Colorless oil, $3 \mathrm{~h}, 0.97 \mathrm{~g}, 85 \%$ yield. ${ }^{1} \mathrm{H}$ NMR (400 MHz, $\left.\mathrm{CDCl}_{3}\right)$ ) : 7.36-7.29 (m, $3 \mathrm{H}), 7.22-7.17(\mathrm{~m}, 2 \mathrm{H}), 4.28$ (q, $J=7.2 \mathrm{~Hz}, 2 \mathrm{H}), 3.93(\mathrm{q}, J=7.2 \mathrm{~Hz}, 2 \mathrm{H}), 2.79-2.70$ (m, 2H), 1.47-1.36 (m, 2H), $1.32(\mathrm{t}, J=7.2 \mathrm{~Hz}, 3 \mathrm{H}), 0.97-0.86(\mathrm{~m}, 6 \mathrm{H}) ;{ }^{13} \mathrm{C} \mathrm{NMR}$ $\left(101 \mathrm{MHz}, \mathrm{CDCl}_{3}\right) \delta: 166.1,164.8,159.7,140.2,128.3,128.1,127.0,126.2,61.1$, 60.9, 37.7, 21.2, 14.1, 14.0, 13.6; HRMS (ESI) m/z: $[\mathrm{M}+\mathrm{Na}]^{+}$calcd for $\mathrm{C}_{17} \mathrm{H}_{22} \mathrm{O}_{4} \mathrm{Na}$ 313.1410; Found 313.1415 .

\section{Diethyl-2-(1-(4-fluorophenyl)ethylidene)malonate (2d)}<smiles>CCOC(=O)C(C(=O)OCC)=C(C)c1ccc(F)cc1</smiles>

Colorless oil, $6 \mathrm{~h}, 0.55 \mathrm{~g}, 49 \%$ yield. ${ }^{1} \mathrm{H}$ NMR (400 MHz, $\left.\mathrm{CDCl}_{3}\right)$ ): 7.27-7.21 $(\mathrm{m}, 2 \mathrm{H}), 7.06-6.99(\mathrm{~m}, 2 \mathrm{H}), 4.27(\mathrm{q}, J=7.2 \mathrm{~Hz}, 2 \mathrm{H}), 3.99(\mathrm{q}, J=7.2 \mathrm{~Hz}, 2 \mathrm{H})$, $2.41(\mathrm{~s}, 3 \mathrm{H}), 1.30(\mathrm{t}, J=7.2 \mathrm{~Hz}, 3 \mathrm{H}), 1.00(\mathrm{t}, J=7.2 \mathrm{~Hz}, 3 \mathrm{H}) ;{ }^{13} \mathrm{C}$ NMR $(101$ $\left.\mathrm{MHz}, \mathrm{CDCl}_{3}\right) \delta: 166.0,164.7,162.7\left(\mathrm{C}-\mathrm{F},{ }^{1} J_{\mathrm{C}-\mathrm{F}}=248.2 \mathrm{~Hz}\right), 154.4,137.4\left(\mathrm{C}-\mathrm{F},{ }^{4} J_{\mathrm{C}-\mathrm{F}}=3.5 \mathrm{~Hz}\right), 128.6$ $\left(\mathrm{C}-\mathrm{F},{ }^{3} J_{\mathrm{C}-\mathrm{F}}=8.2 \mathrm{~Hz}\right), 126.5,115.3\left(\mathrm{C}-\mathrm{F},{ }^{2} J_{\mathrm{C}-\mathrm{F}}=21.4 \mathrm{~Hz}\right), 61.2,61.0,22.9,14.1,13.7 ;$ HRMS (ESI) m/z: $[\mathrm{M}+\mathrm{Na}]^{+}$calcd for $\mathrm{C}_{15} \mathrm{H}_{17} \mathrm{FO}_{4} \mathrm{Na}$ 303.1003; Found 303.1008.

\section{Diethyl-2-(1-(4-chlorophenyl)ethylidene)malonate (2e)}<smiles>CCOC(=O)C(C(=O)OCC)=C(c1ccc(Cl)cc1)[N+](=O)[O-]</smiles>
Colorless oil, $6 \mathrm{~h}, 0.75 \mathrm{~g}, 63 \%$ yield. ${ }^{1} \mathrm{H}$ NMR $\left(400 \mathrm{MHz}, \mathrm{CDCl}_{3}\right) \delta: 7.18(\mathrm{~d}, J=$ $8.4 \mathrm{~Hz}, 2 \mathrm{H}), 7.06(\mathrm{~d}, J=8.4 \mathrm{~Hz}, 2 \mathrm{H}), 4.14(\mathrm{q}, J=7.2 \mathrm{~Hz}, 2 \mathrm{H}), 3.86(\mathrm{q}, J=7.2$ $\mathrm{Hz}, 2 \mathrm{H}), 2.26$ (s, 3H), $1.16(\mathrm{t}, J=7.2 \mathrm{~Hz}, 3 \mathrm{H}), 0.87(\mathrm{t}, J=7.2 \mathrm{~Hz}, 3 \mathrm{H}) ;{ }^{13} \mathrm{C} \mathrm{NMR}$ $\left(101 \mathrm{MHz}, \mathrm{CDCl}_{3}\right)$ 8: 165.8, 164.6, 154.2, 139.9, 134.5, 128.5, 128.1, 126.6, 61.2, 61.1, 22.8, 14.1, 13.7; HRMS (ESI) m/z: [M+Na] $]^{+}$calcd for $\mathrm{C}_{15} \mathrm{H}_{17} \mathrm{ClO}_{4} \mathrm{Na} 319.0708$; Found 319.0712 .

\section{Diethyl-2-(1-(4-bromophenyl)ethylidene)malonate (2f)}<smiles>CCOC(=O)C(C(=O)OCC)=C(c1ccc(Br)cc1)[N+](=O)[O-]</smiles>

Colorless oil, $6 \mathrm{~h}, 0.34 \mathrm{~g}, 25 \%$ yield. ${ }^{1} \mathrm{H}$ NMR (400 MHz, $\left.\mathrm{CDCl}_{3}\right) \delta: 7.47$ (d, $J=$ $8.0 \mathrm{~Hz}, 2 \mathrm{H}), 7.04(\mathrm{~d}, J=8.0 \mathrm{~Hz}, 2 \mathrm{H}), 4.22(\mathrm{q}, J=7.2 \mathrm{~Hz}, 2 \mathrm{H}), 3.93$ (q, $J=7.2$ $\mathrm{Hz}, 2 \mathrm{H}), 2.32(\mathrm{~s}, 3 \mathrm{H}), 1.25$ (t, $J=7.2 \mathrm{~Hz}, 3 \mathrm{H}), 0.95(\mathrm{t}, J=7.2 \mathrm{~Hz}, 3 \mathrm{H}) ;{ }^{13} \mathrm{C} \mathrm{NMR}$ 
(101 MHz, $\left.\mathrm{CDCl}_{3}\right)$ 8: 165.8, 164.6, 154.1, 140.4, 131.5, 128.4, 126.6, 122.6, 61.2, 61.1, 22.7, 14.1, 13.7; HRMS (ESI) m/z: [M+Na] $]^{+}$calcd for $\mathrm{C}_{15} \mathrm{H}_{17} \mathrm{BrO}_{4} \mathrm{Na}$ 363.0202; Found 363.0207.

Diethyl-2-(1-(4-(trifluoromethyl)phenyl)ethylidene)malonate (2g)

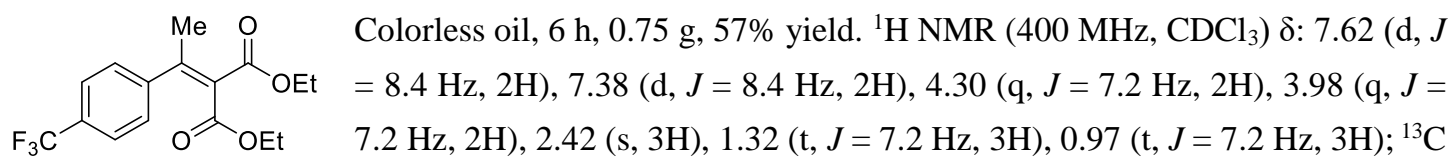
$\operatorname{NMR}\left(101 \mathrm{MHz}, \mathrm{CDCl}_{3}\right) \delta: 165.1,164.3,153.4,145.2\left(\mathrm{C}-\mathrm{F},{ }^{4} J_{\mathrm{C}-\mathrm{F}}=1.4 \mathrm{~Hz}\right), 130.2\left(\mathrm{C}-\mathrm{F},{ }^{2} J_{\mathrm{C}-\mathrm{F}}=32.7\right.$ $\mathrm{Hz}), 127.1,127.0,125.1\left(\mathrm{C}-\mathrm{F},{ }^{3} J_{\mathrm{C}-\mathrm{F}}=3.8 \mathrm{~Hz}\right), 123.9\left(\mathrm{C}-\mathrm{F},{ }^{1} J_{\mathrm{C}-\mathrm{F}}=267.7 \mathrm{~Hz}\right), 61.1,60.9,22.4,13.8,13.3$; HRMS (ESI) m/z: [M+Na] $]^{+}$calcd for $\mathrm{C}_{16} \mathrm{H}_{17} \mathrm{~F}_{3} \mathrm{O}_{4} \mathrm{Na}$ 353.0971; Found 353.0976.

Diethyl-2-(1-(4-(ethoxycarbonyl)phenyl)ethylidene)malonate (2h)

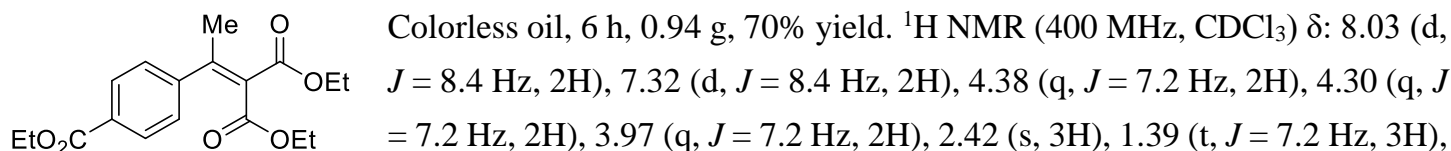
$1.32(\mathrm{t}, J=7.2 \mathrm{~Hz}, 3 \mathrm{H}), 0.99$ (t, $J=7.2 \mathrm{~Hz}, 3 \mathrm{H}) ;{ }^{13} \mathrm{C} \mathrm{NMR}\left(101 \mathrm{MHz}, \mathrm{CDCl}_{3}\right) \delta: 165.8,165.3,164.3$, 154.0, 146.0, 130.2, 129.4, 126.8, 126.6, 61.0, 60.9, 22.4, 14.2, 14.0, 13.5; HRMS (ESI) m/z: [M+Na ${ }^{+}$ calcd for $\mathrm{C}_{18} \mathrm{H}_{22} \mathrm{O}_{6} \mathrm{Na} 357.1309$; Found 357.1312.

Diethyl-2-(1-([1,1'-biphenyl]-4-yl)ethylidene)malonate (2i)

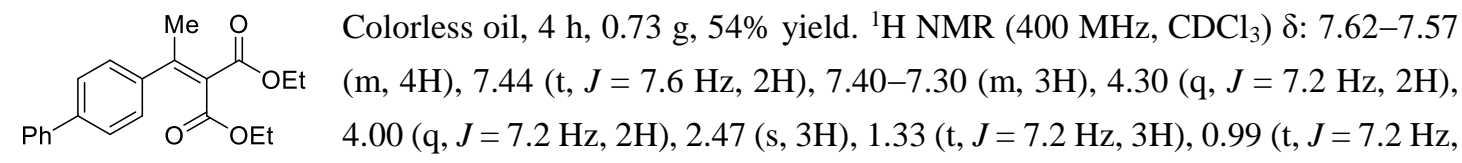
$3 \mathrm{H}) ;{ }^{13} \mathrm{C}$ NMR (101 MHz, $\left.\mathrm{CDCl}_{3}\right) \delta: 166.3,164.8,155.4,141.4,140.5,128.9,127.6,127.2,127.1,127.0$, 126.2, 120.6, 61.2, 61.0, 22.8, 14.2, 13.7; HRMS (ESI) m/z: [M+Na] ${ }^{+}$calcd for $\mathrm{C}_{21} \mathrm{H}_{22} \mathrm{O}_{4} \mathrm{Na}$ 361.1410; Found 361.1415 .

\section{Diethyl-2-(1-(p-tolyl)ethylidene)malonate (2j) ${ }^{7}$}

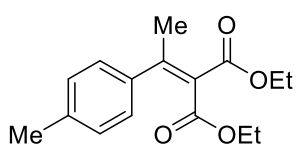

Colorless oil, $6 \mathrm{~h}, 0.63 \mathrm{~g}, 57 \%$ yield. ${ }^{1} \mathrm{H}$ NMR $\left(400 \mathrm{MHz}, \mathrm{CDCl}_{3}\right) \delta: 7.13(\mathrm{~s}, 4 \mathrm{H})$, $4.25(\mathrm{q}, J=7.2 \mathrm{~Hz}, 2 \mathrm{H}), 3.97(\mathrm{q}, J=7.2 \mathrm{~Hz}, 2 \mathrm{H}), 2.41(\mathrm{~s}, 3 \mathrm{H}), 2.32(\mathrm{~s}, 3 \mathrm{H}), 1.29$ $(\mathrm{t}, J=7.2 \mathrm{~Hz}, 3 \mathrm{H}), 0.98(\mathrm{t}, J=7.2 \mathrm{~Hz}, 3 \mathrm{H})$ (the spectra are in accordance with those of the compound reported in the literature).

\section{Diethyl-2-(1-(4-methoxyphenyl)ethylidene)malonate (2k) ${ }^{8}$}

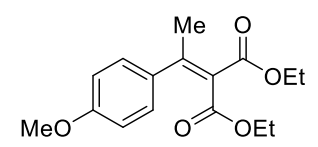

Colorless oil, 6 h, $0.55 \mathrm{~g}, 47 \%$ yield. ${ }^{1} \mathrm{H}$ NMR (400 MHz, $\left.\mathrm{CDCl}_{3}\right)$ ): 7.21 (d, $J=$ $8.8 \mathrm{~Hz}, 2 \mathrm{H}), 6.87(\mathrm{~d}, J=8.8 \mathrm{~Hz}, 2 \mathrm{H}), 4.28(\mathrm{q}, J=7.2 \mathrm{~Hz}, 2 \mathrm{H}), 4.02(\mathrm{q}, J=7.2$ $\mathrm{Hz}, 2 \mathrm{H}), 3.81(\mathrm{~s}, 3 \mathrm{H}), 2.43(\mathrm{~s}, 3 \mathrm{H}), 1.32(\mathrm{t}, J=7.2 \mathrm{~Hz}, 3 \mathrm{H}), 1.04(\mathrm{t}, J=7.2 \mathrm{~Hz}$, $3 \mathrm{H})$ (the spectra are in accordance with those of the compound reported in the literature).

\section{Diethyl-2-(1-(3-fluorophenyl)ethylidene)malonate (2l)}<smiles>CCOC(=O)C(C(=O)OCC)=C(C)c1cccc(F)c1</smiles>

Colorless oil, 6 h, $0.69 \mathrm{~g}, 62 \%$ yield. ${ }^{1} \mathrm{H}$ NMR $\left(400 \mathrm{MHz}, \mathrm{CDCl}_{3}\right) \delta: 7.34-7.26$ $(\mathrm{m}, 1 \mathrm{H}), 7.05-7.94(\mathrm{~m}, 3 \mathrm{H}), 4.28(\mathrm{q}, J=7.1 \mathrm{~Hz}, 2 \mathrm{H}), 3.99(\mathrm{q}, J=7.1 \mathrm{~Hz}, 2 \mathrm{H})$, $2.41(\mathrm{~s}, 3 \mathrm{H}), 1.31(\mathrm{t}, J=7.1 \mathrm{~Hz}, 3 \mathrm{H}), 1.00(\mathrm{t}, J=7.1 \mathrm{~Hz}, 3 \mathrm{H}) ;{ }^{13} \mathrm{C}$ NMR $(101$ $\left.\mathrm{MHz}, \mathrm{CDCl}_{3}\right) \delta: 165.6,164.4,162.4\left(\mathrm{C}-\mathrm{F},{ }^{1} J_{\mathrm{C}-\mathrm{F}}=248.5 \mathrm{~Hz}\right), 153.6,143.6\left(\mathrm{C}-\mathrm{F},{ }^{3} J_{\mathrm{C}-\mathrm{F}}=8.1 \mathrm{~Hz}\right), 129.9$ 
$\left(\mathrm{C}-\mathrm{F},{ }^{3} J_{\mathrm{C}-\mathrm{F}}=9.1 \mathrm{~Hz}\right), 126.8,122.4\left(\mathrm{C}-\mathrm{F},{ }^{4} J_{\mathrm{C}-\mathrm{F}}=3.0 \mathrm{~Hz}\right), 115.1\left(\mathrm{C}-\mathrm{F},{ }^{2} J_{\mathrm{C}-\mathrm{F}}=21.2 \mathrm{~Hz}\right), 113.7\left(\mathrm{C}-\mathrm{F},{ }^{2} J_{\mathrm{C}-\mathrm{F}}\right.$ $=22.2 \mathrm{~Hz}), 61.0,60.9,22.4,14.0,13.5$; HRMS $(\mathrm{ESI}) \mathrm{m} / \mathrm{z}:[\mathrm{M}+\mathrm{Na}]^{+}$calcd for $\mathrm{C}_{15} \mathrm{H}_{17} \mathrm{FO}_{4} \mathrm{Na}_{303.1003}$; Found 303.1008.

\section{Diethyl-2-(1-(m-tolyl)ethylidene)malonate (2m)}<smiles>CCOC(=O)C(C(=O)OCC)=C(C)c1cccc(C)c1</smiles>

Colorless oil, $6 \mathrm{~h}, 0.56 \mathrm{~g}, 51 \%$ yield. ${ }^{1} \mathrm{H}$ NMR (400 $\left.\mathrm{MHz}, \mathrm{CDCl}_{3}\right) \delta: 7.23(\mathrm{t}, J=$ $7.6 \mathrm{~Hz}, 1 \mathrm{H}), 7.05(\mathrm{~s}, 1 \mathrm{H}), 7.13(\mathrm{~d}, J=7.6 \mathrm{~Hz}, 1 \mathrm{H}), 7.04(\mathrm{~d}, J=9.5 \mathrm{~Hz}, 2 \mathrm{H})$, $4.28(\mathrm{q}, J=7.2 \mathrm{~Hz}, 2 \mathrm{H}), 3.97(\mathrm{q}, J=7.2 \mathrm{~Hz}, 2 \mathrm{H}), 2.43(\mathrm{~s}, 3 \mathrm{H}), 2.34(\mathrm{~s}, 3 \mathrm{H})$, $1.32(\mathrm{t}, J=7.2,3 \mathrm{H}), 0.98(\mathrm{t}, J=7.2,3 \mathrm{H}) ;{ }^{13} \mathrm{C} \mathrm{NMR}\left(101 \mathrm{MHz}, \mathrm{CDCl}_{3}\right) \delta: 166.3$,

164.8, 156.0, 141.6, 137.9, 129.2, 128.2, 127.2, 126.0, 123.7, 61.0, 60.9, 22.8, 21.4, 14.1, 13.6; HRMS (ESI) $\mathrm{m} / \mathrm{z}:[\mathrm{M}+\mathrm{Na}]^{+}$calcd for $\mathrm{C}_{16} \mathrm{H}_{20} \mathrm{O}_{4} \mathrm{Na} 299.1254$; Found 299.1260.

\section{Diethyl-2-(1-(3-methoxyphenyl)ethylidene)malonate (2n)}

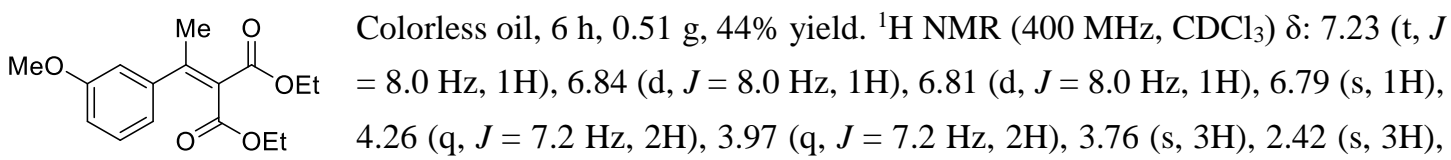
$1.30(\mathrm{t}, J=7.2 \mathrm{~Hz}, 3 \mathrm{H}), 0.97(\mathrm{t}, J=7.2 \mathrm{~Hz}, 3 \mathrm{H}) ;{ }^{13} \mathrm{C} \mathrm{NMR}\left(101 \mathrm{MHz}, \mathrm{CDCl}_{3}\right) \delta: 166.2,164.7,159.4$, 155.5, 143.0, 129.4, 126.2, 119.0, 114.1, 112.1, 61.1, 61.0, 55.3, 22.7, 14.1, 13.6; HRMS (ESI) m/z: $[\mathrm{M}+\mathrm{Na}]^{+}$calcd for $\mathrm{C}_{16} \mathrm{H}_{20} \mathrm{O}_{5} \mathrm{Na} 315.1203$; Found 315.1208 .

\section{Diethyl-2-(1-(2-fluorophenyl)ethylidene)malonate (2o)}<smiles>CCOC(=O)C(C(=O)OCC)=C(C)c1ccccc1F</smiles>
Colorless oil, 4 h, $0.75 \mathrm{~g}, 67 \%$ yield. ${ }^{1} \mathrm{H}$ NMR (400 MHz, $\left.\mathrm{CDCl}_{3}\right) \delta: 7.35-7.27$ (m, $1 \mathrm{H}), 7.18-7.03(\mathrm{~m}, 3 \mathrm{H}), 4.31(\mathrm{q}, J=7.2 \mathrm{~Hz}, 2 \mathrm{H}), 3.97(\mathrm{q}, J=7.2 \mathrm{~Hz}, 2 \mathrm{H}), 2.36(\mathrm{~s}$, $3 \mathrm{H}), 1.33(\mathrm{t}, J=7.2 \mathrm{~Hz}, 3 \mathrm{H}), 0.97(\mathrm{t}, J=7.2 \mathrm{~Hz}, 3 \mathrm{H}) ;{ }^{13} \mathrm{C} \mathrm{NMR}\left(101 \mathrm{MHz}, \mathrm{CDCl}_{3}\right)$ $\delta: 164.9,164.8,158.6\left(\mathrm{C}-\mathrm{F},{ }^{1} J_{\mathrm{C}-\mathrm{F}}=248.5 \mathrm{~Hz}\right), 149.9,130.0\left(\mathrm{C}-\mathrm{F},{ }^{3} J_{\mathrm{C}-\mathrm{F}}=8.1 \mathrm{~Hz}\right)$, $128.9\left(\mathrm{C}-\mathrm{F},{ }^{2} J_{\mathrm{C}-\mathrm{F}}=16.2 \mathrm{~Hz}\right), 128.6\left(\mathrm{C}-\mathrm{F},{ }^{3} J_{\mathrm{C}-\mathrm{F}}=4.0 \mathrm{~Hz}\right), 128.0,123.9\left(\mathrm{C}-\mathrm{F},{ }^{4} J_{\mathrm{C}-\mathrm{F}}=4.0 \mathrm{~Hz}\right), 115.6(\mathrm{C}-\mathrm{F}$, $\left.{ }^{2} J_{\mathrm{C}-\mathrm{F}}=22.2 \mathrm{~Hz}\right), 61.3,60.9,22.4\left(\mathrm{C}-\mathrm{F},{ }^{4} J_{\mathrm{C}-\mathrm{F}}=1.7 \mathrm{~Hz}\right), 14.1,13.6$; HRMS (ESI) m/z: $[\mathrm{M}+\mathrm{Na}]^{+}$calcd for $\mathrm{C}_{15} \mathrm{H}_{17} \mathrm{FO}_{4} \mathrm{Na}$ 303.1003; Found 303.1007.

\section{Diethyl-2-(1-(o-tolyl)ethylidene)malonate (2p)}<smiles>CCOC(=O)C(C(=O)OCC)=C(C)c1ccccc1C</smiles>

Colorless oil, 6 h, $0.49 \mathrm{~g}, 44 \%$ yield. ${ }^{1} \mathrm{H}$ NMR (400 MHz, $\left.\mathrm{CDCl}_{3}\right) \delta: 7.23-7.11(\mathrm{~m}$, $3 \mathrm{H}), 6.99(\mathrm{~d}, J=7.6 \mathrm{~Hz}, 1 \mathrm{H}), 4.35-4.25(\mathrm{~m}, 2 \mathrm{H}), 3.94-3.84(\mathrm{~m}, 2 \mathrm{H}), 2.33(\mathrm{~s}, 3 \mathrm{H})$, $2.26(\mathrm{~s}, 3 \mathrm{H}), 1.33(\mathrm{t}, J=7.2 \mathrm{~Hz}, 3 \mathrm{H}), 0.89(\mathrm{t}, J=7.2 \mathrm{~Hz}, 3 \mathrm{H}) ;{ }^{13} \mathrm{C} \mathrm{NMR}(101 \mathrm{MHz}$, $\left.\mathrm{CDCl}_{3}\right) \delta: 165.3,164.7,156.3,141.1,133.8,130.0,127.8,126.7,126.2,125.5,61.0$, 60.7, 22.7, 19.1, 14.1, 13.5; HRMS (ESI) m/z: [M+Na] $]^{+}$calcd for $\mathrm{C}_{16} \mathrm{H}_{20} \mathrm{O}_{4} \mathrm{Na}$ 299.1254; Found 299.1258.

\section{Diethyl-2-(1-(benzo $[d][1,3]$ dioxol-5-yl)ethylidene)malonate (2q)}<smiles>CCOC(=O)C(C(=O)OCC)=C(C)c1ccc2c(c1)OCO2</smiles>

Colorless oil, 6 h, $0.76 \mathrm{~g}, 62 \%$ yield. ${ }^{1} \mathrm{H}$ NMR (400 MHz, $\left.\mathrm{CDCl}_{3}\right) \delta: 6.80-6.70$ $(\mathrm{m}, 3 \mathrm{H}), 5.95(\mathrm{~s}, 2 \mathrm{H}), 4.26(\mathrm{q}, J=7.2 \mathrm{~Hz}, 2 \mathrm{H}), 4.04(\mathrm{q}, J=7.2 \mathrm{~Hz}, 2 \mathrm{H}), 2.40$ (s, $3 \mathrm{H}), 1.30(\mathrm{t}, J=7.2 \mathrm{~Hz}, 3 \mathrm{H}), 1.07(\mathrm{t}, J=7.2 \mathrm{~Hz}, 3 \mathrm{H}) ;{ }^{13} \mathrm{C} \mathrm{NMR}(101 \mathrm{MHz}$, $\left.\mathrm{CDCl}_{3}\right) \delta:$ 166.3, 164.6, 155.0, 147.9, 147.6, 135.3, 125.9, 120.6, 108.0, 107.4, 101.3, 60.9 (2), 22.6, 14.0, 13.7; HRMS (ESI) m/z: [M+Na] ${ }^{+}$calcd for $\mathrm{C}_{16} \mathrm{H}_{18} \mathrm{O}_{6} \mathrm{Na} 329.0996$; Found 329.1001.

\section{Diethyl-2-(1-(naphthalen-1-yl)ethylidene)malonate (2r)}




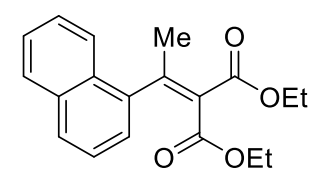

Colorless oil, $6 \mathrm{~h}, 0.51 \mathrm{~g}, 41 \%$ yield. ${ }^{1} \mathrm{H}$ NMR (400 MHz, $\left.\mathrm{CDCl}_{3}\right) \delta: 7.88-7.82$ $(\mathrm{m}, 1 \mathrm{H}), 7.78(\mathrm{t}, J=8.0 \mathrm{~Hz}, 2 \mathrm{H}), 7.53-7.45(\mathrm{~m}, 2 \mathrm{H}), 7.45-7.39(\mathrm{~m}, 1 \mathrm{H}), 7.25-$ $7.09(\mathrm{dd}, J=7.0,0.6 \mathrm{~Hz}, 1 \mathrm{H}), 4.35(\mathrm{qd}, J=7.2,1.6 \mathrm{~Hz}, 2 \mathrm{H}), 3.80-3.62(\mathrm{~m}, 2 \mathrm{H})$, $2.50(\mathrm{~s}, 3 \mathrm{H}), 1.37(\mathrm{t}, J=7.2 \mathrm{~Hz}, 3 \mathrm{H}), 0.57(\mathrm{t}, J=7.2 \mathrm{~Hz}, 3 \mathrm{H}) ;{ }^{13} \mathrm{C}$ NMR $(101$ $\left.\mathrm{MHz}, \mathrm{CDCl}_{3}\right) \delta: 165.2,164.9,155.3,139.3,133.4,129.8,128.4,128.2,128.0,126.4,126.0,125.2,125.1$,

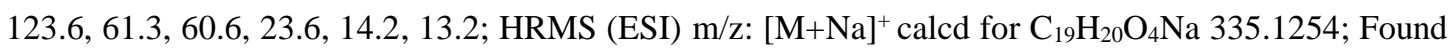
335.1258 .

Diethyl-2-(1-(naphthalen-2-yl)ethylidene)malonate (2s)<smiles>CCOC(=O)C(C(=O)OCC)=C(C)c1ccc2ccccc2c1</smiles>
Colorless oil, $4 \mathrm{~h}, 0.72 \mathrm{~g}, 58 \%$ yield. ${ }^{1} \mathrm{H}$ NMR (400 MHz, $\left.\mathrm{CDCl}_{3}\right) \delta: 7.84-7.75$ $(\mathrm{m}, 7 \mathrm{H}), 7.71(\mathrm{~d}, J=2.0 \mathrm{~Hz}, 1 \mathrm{H}), 7.50-7.42(\mathrm{~m}, 2 \mathrm{H}), 7.35(\mathrm{dd}, J=8.4,2.0 \mathrm{~Hz}$, $1 \mathrm{H}), 4.30(\mathrm{q}, J=7.2 \mathrm{~Hz}, 2 \mathrm{H}), 3.92(\mathrm{q}, J=7.2 \mathrm{~Hz}, 2 \mathrm{H}), 2.51(\mathrm{~s}, 3 \mathrm{H}), 1.32(\mathrm{t}, J$ $=7.2 \mathrm{~Hz}, 3 \mathrm{H}), 0.85(\mathrm{t}, J=7.2 \mathrm{~Hz}, 3 \mathrm{H}) ;{ }^{13} \mathrm{C} \mathrm{NMR}\left(101 \mathrm{MHz}, \mathrm{CDCl}_{3}\right) \delta: 166.2$, 164.8, 155.6, 139.0, 133.0, 132.9, 128.2, 128.1, 127.7, 126.6, 126.6, 126.5, 125.8, 124.6, 61.1, 61.0, 22.8, 14.2, 13.6; HRMS (ESI) m/z: $[\mathrm{M}+\mathrm{Na}]^{+}$calcd for $\mathrm{C}_{19} \mathrm{H}_{20} \mathrm{O}_{4} \mathrm{Na} 335.1254$; Found 335.1259.

\section{Diethyl-2-(1-(furan-2-yl)ethylidene)malonate (2u)}<smiles>CCOC(=O)C(C(=O)OCC)=C(C)c1ccco1</smiles>

Light yellow oil, 4 h, $0.39 \mathrm{~g}, 39 \%$ yield. ${ }^{1} \mathrm{H}$ NMR (400 MHz, $\left.\mathrm{CDCl}_{3}\right) \delta: 7.46(\mathrm{~s}, 1 \mathrm{H})$, $6.76(\mathrm{~d}, J=3.6 \mathrm{~Hz}, 1 \mathrm{H}), 6.50-6.45(\mathrm{~m}, 1 \mathrm{H}), 4.33(\mathrm{q}, J=7.2 \mathrm{~Hz}, 2 \mathrm{H}), 4.24(\mathrm{q}, J=$ $7.2 \mathrm{~Hz}, 2 \mathrm{H}), 2.48$ (s, 3H), 1.35-1.27 (m, 6H); $\left.{ }^{13} \mathrm{C} \mathrm{NMR} \mathrm{(101} \mathrm{MHz,} \mathrm{CDCl}_{3}\right) \delta: 167.6$, 164.4, 152.2, 144.7, 139.1, 121.1, 114.5, 112.1, 61.3, 60.9, 16.9, 14.1, 14.0; HRMS (ESI) $\mathrm{m} / \mathrm{z}$ : $[\mathrm{M}+\mathrm{Na}]^{+}$calcd for $\mathrm{C}_{13} \mathrm{H}_{16} \mathrm{O}_{5} \mathrm{Na} 275.0890$; Found 275.0895.

Method B: The $\beta$-dialkyl and thienyl substituted $\alpha, \beta$-unsaturated malonates $\mathbf{2 t}$ and $\mathbf{2 v}$ were prepared by indium-mediated Knoevenagel condensation of malonates to terminal alkynes according to literature method. ${ }^{3}$

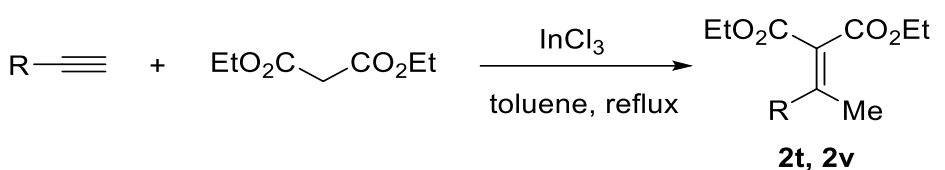

General procedure: To a solution of indium (III) chloride $(220 \mathrm{mg}, 1.0 \mathrm{mmol})$ in toluene $(10 \mathrm{~mL})$ was added diethyl malonate $(1.60 \mathrm{~g}, 10 \mathrm{mmol})$ and terminal alkynes $(20 \mathrm{mmol})$. The resulting mixture was then heated to $110{ }^{\circ} \mathrm{C}$ in an oil bath and stirred at the same temperature for $20 \mathrm{~h}$. The reaction mixture was then concentrated in vacuo, and the residue was purified by flash chromatography on a silica-gel with petroleum ether/ethyl acetate (10:1) as an eluent to give the corresponding desired product $\mathbf{2 t}$ and 2v as light yellow or colorless oil.

\section{Diethyl-2-(1-(thiophen-2-yl)ethylidene)malonate (2t)}

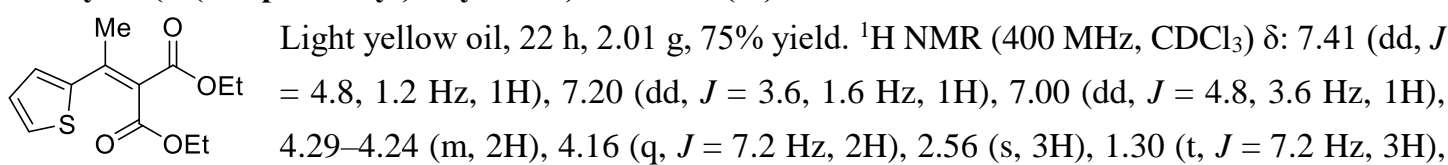
$1.17(\mathrm{t}, J=7.2 \mathrm{~Hz}, 3 \mathrm{H}) ;{ }^{13} \mathrm{C} \mathrm{NMR}\left(101 \mathrm{MHz}, \mathrm{CDCl}_{3}\right) \delta: 167.1,164.2,146.7,142.7,128.1,128.0,127.3$, 124.7, 61.4, 61.1, 22.7, 14.1, 13.8; HRMS (ESI) m/z: [M+Na $]^{+}$calcd for $\mathrm{C}_{13} \mathrm{H}_{16} \mathrm{O}_{4} \mathrm{SNa} 291.0662$; Found 291.0665 . 


\section{Diethyl-2-(1-cyclohexylethylidene)malonate (2v)}<smiles>CCOC(=O)C(C(=O)OCC)=C(C)C1CCCCC1</smiles>

Colorless oil, $22 \mathrm{~h}, 1.64 \mathrm{~g}, 61 \%$ yield. ${ }^{1} \mathrm{H}$ NMR (400 MHz, $\left.\mathrm{CDCl}_{3}\right) \delta: 4.17-4.07$ (m, $4 \mathrm{H}), 2.65-2.54(\mathrm{~m}, 1 \mathrm{H}), 2.00(\mathrm{~s}, 3 \mathrm{H}), 1.71-1.64(\mathrm{~m}, 2 \mathrm{H}), 1.61-1.51(\mathrm{~m}, 3 \mathrm{H}), 1.29$ $1.14(\mathrm{~m}, 11 \mathrm{H}), 1.12-1.01(\mathrm{~m}, 1 \mathrm{H}) ;{ }^{13} \mathrm{C} \mathrm{NMR}\left(101 \mathrm{MHz}, \mathrm{CDCl}_{3}\right) \delta: 165.9,165.2$, 162.0, 123.7, 60.6, 60.5, 44.1, 30.0, 25.9, 25.8, 15.7, 14.0, 13.9; HRMS (ESI) m/z:

$[\mathrm{M}+\mathrm{Na}]^{+}$calcd for $\mathrm{C}_{15} \mathrm{H}_{24} \mathrm{O}_{4} \mathrm{Na} 291.1567$; Found 291.1573 .

\section{(C) Asymmetric Hydrogenation of $\beta$-Aryl Alkylidene Malonate Esters}

\section{Asymmetric hydrogenation of 2 a for optimization the reaction conditions.}<smiles>CCOC(=O)C(C(=O)OCC)=C(C)c1ccccc1</smiles>

2a

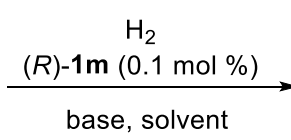

base, solvent<smiles>CCOC(=O)C(C(=O)OCC)C(C)=C(C)c1ccccc1</smiles>

3a

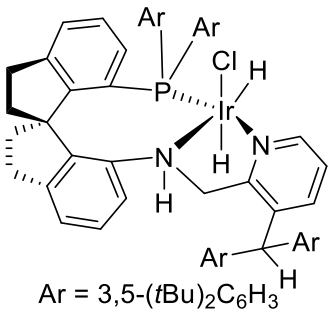

$(R)-1 \mathrm{~m}$

General procedure: To a $20 \mathrm{~mL}$ hydrogenation vessel containing $\alpha, \beta$-unsaturated malonate $2 \mathbf{a}(262 \mathrm{mg}$, $1.0 \mathrm{mmol})$ was added a solution of iridium catalyst $(R)-\mathbf{1 m}(1.3 \mathrm{mg}, 0.001 \mathrm{mmol})$ in alcoholic solvent $(1.0 \mathrm{~mL})$, and a solution of base $(0.4 \mathrm{mmol})$ in alcoholic solvent $(1.0 \mathrm{~mL})$ under nitrogen atmosphere. The autoclave was purged with hydrogen by pressurizing to $5 \mathrm{~atm}$ and releasing the pressure. This procedure was repeated three times and pressurized to 30 or $50 \mathrm{~atm}$ of $\mathrm{H}_{2}$. The reaction mixture was then stirred at room temperature or $50^{\circ} \mathrm{C}$ in an oil bath until no obvious hydrogen pressure drop was observed. After releasing the hydrogen pressure, the reaction mixture was filtered through a short silica gel column, and the filtrate was then concentrated in vacuo and chromatographed on a silica-gel with petroleum ether/ethyl acetate $(5: 1-2: 1)$ to afford the product 3a and determine the enantiomeric excess of the product by chiral HPLC. The obtained results are summarized in Table S1.

Table S1. Optimizing the Reaction Conditions of Hydrogenation of 2a With Catalyst $(R)-1 \mathrm{~m}^{a}{ }^{a}$

$\begin{array}{ccccccc}\text { entry } & \text { solvent } & \text { base } & \text { time }(\mathrm{h}) & \text { conv }(\%)^{b} & \text { yield }(\%)^{c} & \text { ee }(\%)^{d} \\ 1 & \text { EtOH } & t \mathrm{BuOK} & 12 & >99 & 99 & 94 \\ 2^{e} & \mathrm{MeOH} & t \mathrm{BuOK} & 12 & 80 & 80 & 88 \\ 3^{e} & n \mathrm{PrOH} & t \mathrm{BuOK} & 12 & >99 & 99 & 93 \\ 4^{e} & i \mathrm{PrOH} & t \mathrm{BuOK} & 12 & 95 & 93 & 93 \\ 5 & \mathrm{EtOH} & t \mathrm{BuONa} & 12 & >99 & 99 & 93 \\ 6 & \mathrm{EtOH} & \mathrm{K}_{2} \mathrm{CO}_{3} & 24 & 85 & 83 & 90 \\ 7^{f} & \mathrm{EtOH} & t \mathrm{BuOK} & 12 & 98 & 97 & 94 \\ 8^{g} & \mathrm{EtOH} & t \mathrm{BuOK} & 12 & 87 & 87 & 93 \\ 9^{h} & \mathrm{EtOH} & t \mathrm{BuOK} & 8 & >99 & 98 & 88 \\ 10^{i} & \mathrm{EtOH} & t \mathrm{BuOK} & 10 & >99 & 99 & 94\end{array}$




$\begin{array}{lllllll}11^{j} & \mathrm{EtOH} & t \mathrm{BuOK} & 24 & >99 & 99 & 92 \\ 12^{k} & \mathrm{EtOH} & t \mathrm{BuOK} & 30 & 99 & 98 & 94 \\ 13^{l} & \mathrm{EtOH} & t \mathrm{BuOK} & 66 & 98 & 96 & 93 \\ 14^{m} & \mathrm{EtOH} & t \mathrm{BuOK} & 6 \mathrm{~d} & 95 & 94 & 94\end{array}$

${ }^{a}$ Reaction conditions: $1.0 \mathrm{mmol} \mathrm{scale},(R)-\mathbf{1 m} / \mathrm{t} \mathrm{BuOK} / \mathbf{2 a}=1: 400: 1000$, EtOH $(2.0 \mathrm{~mL}), 30 \mathrm{~atm} \mathrm{H}_{2}$, room temperature $\left(25-30{ }^{\circ} \mathrm{C}\right) .{ }^{b}$ Determined by ${ }^{1} \mathrm{H}$ NMR. ${ }^{c}$ NMR yield. ${ }^{d}$ Determined by HPLC using chiral column. ${ }^{e}$ Transesterification product was obtained. ${ }^{f} t \mathrm{BuOK} / \mathbf{2 a}=1: 1 .{ }^{g} t \mathrm{BuOK} / \mathbf{2 a}=1: 5 .{ }^{h}$ At $50{ }^{\circ} \mathrm{C} .{ }^{i} 50 \mathrm{~atm} \mathrm{H}{ }_{2}{ }^{j} 10 \mathrm{~atm} \mathrm{H}_{2 .}{ }^{k} 5 \mathrm{mmol} \mathrm{scale}, 0.02 \mathrm{~mol} \%(R)-1 \mathrm{~m} .{ }^{l} 10 \mathrm{mmol} \mathrm{scale}, 0.01 \mathrm{~mol} \%(R)-$ 1m, 80 atm $\mathrm{H}_{2}$ (initial). ${ }^{m} 20 \mathrm{mmol}$ scale, $0.005 \mathrm{~mol} \%(R)-\mathbf{1 m}, 80$ atm $\mathrm{H}_{2}$ (initial).

\section{Asymmetric hydrogenation of $\beta$-aryl alkylidene malonate esters.}
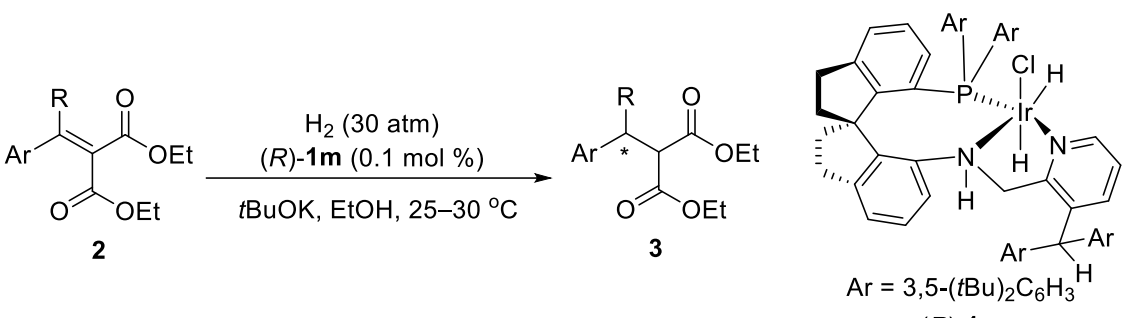

$(R)-1 \mathrm{~m}$

General procedure for $\mathbf{S} / \mathbf{C}=\mathbf{1 , 0 0 0}$ : To a $20 \mathrm{~mL}$ hydrogenation vessel containing $\alpha, \beta$-unsaturated malonate $2(1.0 \mathrm{mmol})$ was added a solution of iridium catalyst $(R)-\mathbf{1 m}(1.3 \mathrm{mg}, 0.001 \mathrm{mmol})$ in EtOH $(1.0 \mathrm{~mL})$, and a solution of $t \mathrm{BuOK}(44.8 \mathrm{mg}, 0.4 \mathrm{mmol})$ in EtOH $(1.0 \mathrm{~mL})$ under nitrogen atmosphere. The autoclave was purged with hydrogen by pressurizing to $5 \mathrm{~atm}$ and releasing the pressure. This procedure was repeated three times and then pressurized to 30 or $50 \mathrm{~atm}$ of $\mathrm{H}_{2}$. The reaction mixture was stirred at room temperature $\left(25-30^{\circ} \mathrm{C}\right)$ until no obvious hydrogen pressure drop was observed. After releasing the hydrogen pressure, the reaction mixture was filtered through a short silica gel column, and the filtrate was then concentrated in vacuo and chromatographed on a silica-gel with petroleum ether/ethyl acetate $(5: 1-2: 1)$ to afford the products 3 and determine the enantiomeric excess of the product by chiral HPLC.

\section{(-)-(R)-Diethyl-2-(1-phenylethyl)malonate (3a) ${ }^{9}$}

Colorless oil, $12 \mathrm{~h}, 256 \mathrm{mg}, 97 \%$ yield, $R_{\mathrm{f}}=0.7$ (petroleum ether/ethyl acetate $=5: 1$ ),<smiles>CCOC(=O)C(C(=O)OCC)C(C)c1ccccc1</smiles>
$94 \%$ ee. $[\alpha]_{\mathrm{D}}^{23}-24.2\left(c 1.0, \mathrm{CHCl}_{3}\right)$ [the absolute configuration was determined by converting it to ethyl-3-phenylbutanoate and comparing its optical rotation that reported for $(R)$-isomer. $[\alpha]_{\mathrm{D}}^{21}-24.2\left(c 0.94, \mathrm{CHCl}_{3}\right)$, lit. $^{10}[\alpha]_{\mathrm{D}}^{20}-23.5(c) 0.94$, $\left.\mathrm{CHCl}_{3}\right), 91 \%$ ee for $(R)$-isomer]. ${ }^{1} \mathrm{H}$ NMR (400 MHz, $\left.\mathrm{CDCl}_{3}\right) \delta: 7.33-7.16(\mathrm{~m}, 5 \mathrm{H}), 4.24(\mathrm{q}, J=7.2 \mathrm{~Hz}$, 2H), $3.92(\mathrm{qd}, J=7.2,1.6 \mathrm{~Hz}, 2 \mathrm{H}), 3.60(\mathrm{~d}, J=10.8 \mathrm{~Hz}, 1 \mathrm{H}), 3.57-3.47(\mathrm{~m}, 1 \mathrm{H}), 1.32(\mathrm{~d}, J=6.8 \mathrm{~Hz}$, $3 \mathrm{H}), 1.29(\mathrm{t}, J=7.2 \mathrm{~Hz}, 3 \mathrm{H}), 0.97(\mathrm{t}, J=7.2 \mathrm{~Hz}, 3 \mathrm{H})$. HPLC analysis (Chiralpak AD-H column, hexane: isopropanol $=98: 2$; flow rate $=1 \mathrm{~mL} / \mathrm{min}$; UV detection at $210 \mathrm{~nm}): t_{\mathrm{R}}(R)=9.0 \min \left(\right.$ major); $t_{\mathrm{R}}(S)=$ $10.3 \mathrm{~min}$ (minor).

\section{(-)-Diethyl-2-(1-phenylpropyl)malonate (3b) ${ }^{11}$}<smiles>CCOC(=O)C(C(=O)OCC)C(=O)c1ccccc1</smiles>

Colorless oil, $36 \mathrm{~h}, 245 \mathrm{mg}, 88 \%$ yield $(\mathrm{S} / \mathrm{C}=500), R_{\mathrm{f}}=0.6$ (petroleum ether/ethyl acetate $=5: 1), 92 \%$ ee. $[\alpha]_{\mathrm{D}}^{26}-14.6\left(\right.$ c $\left.1.0, \mathrm{CHCl}_{3}\right) .{ }^{1} \mathrm{H}$ NMR $\left(400 \mathrm{MHz}, \mathrm{CDCl}_{3}\right) \delta$ : 7.31-7.24 (dd, $J=8.6,6.6 \mathrm{~Hz}, 2 \mathrm{H}), 7.23-7.15(\mathrm{~m}, 3 \mathrm{H}), 4.24(\mathrm{q}, J=7.2 \mathrm{~Hz}, 2 \mathrm{H})$, 
$3.87(\mathrm{q}, J=7.2 \mathrm{~Hz}, 2 \mathrm{H}), 3.64(\mathrm{~d}, J=11.2 \mathrm{~Hz}, 1 \mathrm{H}), 3.27(\mathrm{td}, J=11.0,3.6 \mathrm{~Hz}, 1 \mathrm{H}), 1.83-1.71(\mathrm{~m}, 1 \mathrm{H})$, $1.64-1.53(\mathrm{~m}, 1 \mathrm{H}), 1.29(\mathrm{t}, J=7.2 \mathrm{~Hz}, 3 \mathrm{H}), 0.93(\mathrm{t}, J=7.2 \mathrm{~Hz}, 3 \mathrm{H}), 0.72(\mathrm{t}, J=7.2 \mathrm{~Hz}, 3 \mathrm{H})$. HPLC analysis (Chiralpak AD-H column, hexane: isopropanol $=98: 2$; flow rate $=1 \mathrm{~mL} / \mathrm{min}$; UV detection at $210 \mathrm{~nm}): t_{\mathrm{R}}($ major $)=10.9 \mathrm{~min} ; t_{\mathrm{R}}($ minor $)=11.5 \mathrm{~min}$.

\section{(-)-Diethyl-2-(1-phenylbutyl)malonate (3c) ${ }^{12}$}

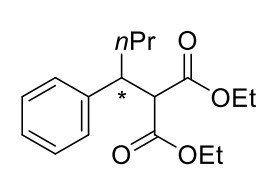

Colorless oil, $48 \mathrm{~h}, 210 \mathrm{mg}, 72 \%$ yield $\left(\mathrm{S} / \mathrm{C}=500,50 \mathrm{~atm} \mathrm{H}_{2}\right), R_{\mathrm{f}}=0.7$ (petroleum ether/ethyl acetate $=5: 1), 74 \%$ ee. $[\alpha]_{\mathrm{D}}^{27}-10.0\left(c 1.0, \mathrm{CHCl}_{3}\right) .{ }^{1} \mathrm{H} \mathrm{NMR}(400 \mathrm{MHz}$, $\left.\mathrm{CDCl}_{3}\right)$ : $: 7.28-7.21(\mathrm{~m}, 2 \mathrm{H}), 7.20-7.13(\mathrm{~m}, 3 \mathrm{H}), 4.22(\mathrm{q}, J=7.2 \mathrm{~Hz}, 2 \mathrm{H}), 3.84$ (q, $J=7.2 \mathrm{~Hz}, 2 \mathrm{H}), 3.60(\mathrm{~d}, J=11.2 \mathrm{~Hz}, 1 \mathrm{H}), 3.34(\mathrm{td}, J=10.4,4.4 \mathrm{~Hz}, 1 \mathrm{H}), 1.64-1.56$ $(\mathrm{m}, 2 \mathrm{H}), 1.27(\mathrm{t}, J=7.2 \mathrm{~Hz}, 3 \mathrm{H}), 1.13-1.02(\mathrm{~m}, 2 \mathrm{H}), 0.90(\mathrm{t}, J=7.2 \mathrm{~Hz}, 3 \mathrm{H}), 0.80(\mathrm{t}, J=7.2 \mathrm{~Hz}, 3 \mathrm{H})$. HPLC analysis (Chiralpak AD-H column, hexane: isopropanol $=95: 5$; flow rate $=1 \mathrm{~mL} / \mathrm{min}$; UV detection at $210 \mathrm{~nm}): t_{\mathrm{R}}($ major $)=5.6 \mathrm{~min} ; t_{\mathrm{R}}($ minor $)=6.9 \mathrm{~min}$.

\section{(-)-(R)-Diethyl-2-(1-(4-fluorophenyl)ethyl)malonate (3d)}

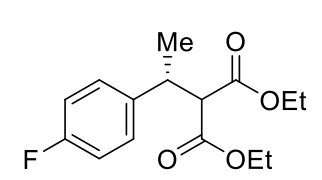

Colorless oil, $12 \mathrm{~h}, 265 \mathrm{mg}, 94 \%$ yield, $R_{\mathrm{f}}=0.7$ (petroleum ether/ethyl acetate $=$ $5: 1), 94 \%$ ee. $[\alpha]_{\mathrm{D}}^{27}-22.0\left(\right.$ c $\left.1.0, \mathrm{CHCl}_{3}\right)$ [the absolute configuration was determined by converting it to ethyl 3-(4-fluorophenyl)butanoate and comparing its optical rotation that reported for $(R)$-isomer, $[\alpha]_{\mathrm{D}}^{29}-21.8\left(c 1.0, \mathrm{CHCl}_{3}\right)$; lit. ${ }^{13}$ $[\alpha]_{\mathrm{D}}^{20}-24.3\left(c 1.0, \mathrm{CHCl}_{3}\right), 99 \%$ ee for $(R)$-isomer]. ${ }^{1} \mathrm{H}$ NMR $\left(400 \mathrm{MHz}, \mathrm{CDCl}_{3}\right) \delta: 7.28-7.21(\mathrm{~m}, 2 \mathrm{H})$, $7.03-6.95(\mathrm{~m}, 2 \mathrm{H}), 4.25$ (q, $J=7.2 \mathrm{~Hz}, 2 \mathrm{H}), 3.95(\mathrm{qd}, J=7.2,1.6 \mathrm{~Hz}, 2 \mathrm{H}), 3.57(\mathrm{~d}, J=6.8 \mathrm{~Hz}, 1 \mathrm{H})$, $3.55-3.48(\mathrm{~m}, 1 \mathrm{H}), 1.34(\mathrm{~d}, J=6.4 \mathrm{~Hz}, 3 \mathrm{H}), 1.30(\mathrm{t}, J=7.2 \mathrm{~Hz}, 3 \mathrm{H}), 1.02(\mathrm{t}, J=7.2 \mathrm{~Hz}, 3 \mathrm{H}) ;{ }^{13} \mathrm{C}$ NMR $\left(101 \mathrm{MHz}, \mathrm{CDCl}_{3}\right) \delta: 168.1,167.7,161.6\left(\mathrm{C}-\mathrm{F},{ }^{1} J_{\mathrm{C}-\mathrm{F}}=254.4 \mathrm{~Hz}\right), 138.8\left(\mathrm{C}-\mathrm{F},{ }^{4} J_{\mathrm{C}-\mathrm{F}}=3.0 \mathrm{~Hz}\right), 129.0(\mathrm{C}-$ $\left.\mathrm{F},{ }^{3} J_{\mathrm{C}-\mathrm{F}}=7.8 \mathrm{~Hz}\right), 115.0\left(\mathrm{C}-\mathrm{F},{ }^{2} J_{\mathrm{C}-\mathrm{F}}=21.4 \mathrm{~Hz}\right), 61.3,61.0,59.2,39.2,20.1,13.9,13.6$; HRMS (ESI) m/z: $[\mathrm{M}+\mathrm{Na}]^{+}$calcd for $\mathrm{C}_{15} \mathrm{H}_{19} \mathrm{FO}_{4} \mathrm{Na}$ 305.1160; Found 305.1164. HPLC analysis (Chiralpak AD-H column, hexane: isopropanol $=90: 10$; flow rate $=1 \mathrm{~mL} / \mathrm{min}$; $\mathrm{UV}$ detection at $210 \mathrm{~nm}$ ): $t_{\mathrm{R}}(R)=5.8 \mathrm{~min}$ (major); $t_{\mathrm{R}}(S)=7.4 \min$ (minor).

\section{(-)-Diethyl-2-(1-(4-chlorophenyl)ethyl)malonate (3e) $)^{14}$}

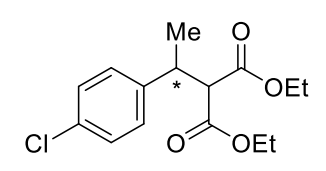

Colorless oil, $12 \mathrm{~h}, 283 \mathrm{mg}, 95 \%$ yield, $R_{\mathrm{f}}=0.7$ (petroleum ether/ethyl acetate $=$ 5:1), $94 \%$ ee. $[\alpha]_{\mathrm{D}}^{25}-19.6\left(c 1.0, \mathrm{CHCl}_{3}\right) .{ }^{1} \mathrm{H} \mathrm{NMR}\left(400 \mathrm{MHz}, \mathrm{CDCl}_{3}\right) \delta: 7.25(\mathrm{~d}$, $J=8.6 \mathrm{~Hz}, 2 \mathrm{H}), 7.18(\mathrm{~d}, J=8.6 \mathrm{~Hz}, 2 \mathrm{H}), 4.23(\mathrm{q}, J=7.2 \mathrm{~Hz}, 2 \mathrm{H}), 3.94(\mathrm{qd}, J=$ 7.2, $2.0 \mathrm{~Hz}, 2 \mathrm{H}), 3.57(\mathrm{~d}, J=10.8 \mathrm{~Hz}, 1 \mathrm{H}), 3.54-2.46(\mathrm{~m}, 1 \mathrm{H}), 1.35-1.23(\mathrm{~m}$, $6 \mathrm{H}), 1.01(\mathrm{t}, J=7.2 \mathrm{~Hz}, 3 \mathrm{H})$. HPLC analysis (Chiralpak AD-H column, hexane: isopropanol = 90:10; flow rate $=1 \mathrm{~mL} / \mathrm{min} ; \mathrm{UV}$ detection at $210 \mathrm{~nm}): t_{\mathrm{R}}($ major $)=6.4 \mathrm{~min} ; t_{\mathrm{R}}($ minor $)=7.7 \mathrm{~min}$.

\section{(-)-Diethyl-2-(1-(4-bromophenyl)ethyl)malonate (3f)}

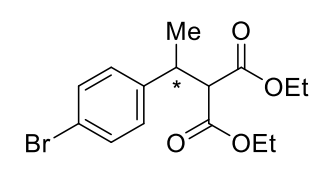

Colorless oil, $12 \mathrm{~h}, 335 \mathrm{mg}, 98 \%$ yield, $R_{\mathrm{f}}=0.7$ (petroleum ether/ethyl acetate $=$ 5:1), $94 \%$ ee. $[\alpha]_{\mathrm{D}}^{24}-15.8\left(c 1.0, \mathrm{CHCl}_{3}\right) ;{ }^{1} \mathrm{H}$ NMR $\left(400 \mathrm{MHz}, \mathrm{CDCl}_{3}\right) \delta: 7.40(\mathrm{~d}$, $J=8.4 \mathrm{~Hz}, 2 \mathrm{H}), 7.12(\mathrm{~d}, J=8.4 \mathrm{~Hz}, 2 \mathrm{H}), 4.22(\mathrm{q}, J=7.2 \mathrm{~Hz}, 2 \mathrm{H}), 3.94(\mathrm{qd}, J=$ $7.2,2.0 \mathrm{~Hz}, 2 \mathrm{H}), 3.57(\mathrm{~d}, J=10.4 \mathrm{~Hz}, 1 \mathrm{H}), 3.54-3.45(\mathrm{~m}, 1 \mathrm{H}), 1.30(\mathrm{~d}, J=6.8$ $\mathrm{Hz}, 3 \mathrm{H}), 1.27$ (t, $J=7.2 \mathrm{~Hz}, 3 \mathrm{H}), 1.01(\mathrm{t}, J=7.2 \mathrm{~Hz}, 3 \mathrm{H}) ;{ }^{13} \mathrm{C} \mathrm{NMR}\left(101 \mathrm{MHz}, \mathrm{CDCl}_{3}\right)$ 8: 168.0, 167.6, 142.2, 131.4, 129.3, 120.5, 61.5, 61.2, 59.0, 39.4, 20.0, 14.1, 13.8; HRMS (ESI) m/z: $[\mathrm{M}+\mathrm{Na}]^{+}$calcd for $\mathrm{C}_{15} \mathrm{H}_{19} \mathrm{BrO}_{4} \mathrm{Na}$ 365.0359; Found 365.0365. HPLC analysis (Chiralpak AD-H column, hexane: 
isopropanol $=90: 10$; flow rate $=1 \mathrm{~mL} / \mathrm{min} ; \mathrm{UV}$ detection at $210 \mathrm{~nm}): t_{\mathrm{R}}($ major $)=6.7 \mathrm{~min} ; t_{\mathrm{R}}($ minor $)=$ 8.1 min.

\section{(-)-(R)-Diethyl-2-(1-(4-(trifluoromethyl)phenyl)ethyl)malonate (3g)}

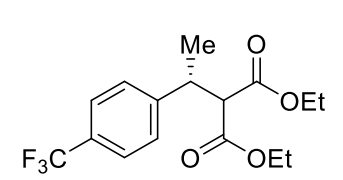

Colorless oil, 12 h, $325 \mathrm{mg}, 98 \%$ yield, $R_{\mathrm{f}}=0.8$ (petroleum ether/ethyl acetate $=5: 1), 96 \%$ ee. $[\alpha]_{\mathrm{D}}^{26}-13.8\left(c 1.0, \mathrm{CHCl}_{3}\right)$ [the absolute configuration was determined by converting it to ethyl 3-(4-(trifluoromethyl)phenyl)butanoate and comparing its optical rotation that reported for $(S)$-isomer, $[\alpha]_{\mathrm{D}}^{25}-20.3(c$ $\left.0.67, \mathrm{CHCl}_{3}\right)$; lit. ${ }^{15}[\alpha]_{\mathrm{D}}^{23}+19.1\left(c 0.67, \mathrm{CHCl}_{3}, 89 \%\right.$ ee for $(S)$-isomer]. ${ }^{1} \mathrm{H}$ NMR $\left(400 \mathrm{MHz}, \mathrm{CDCl}_{3}\right) \delta$ : $7.55(\mathrm{~d}, J=8.1 \mathrm{~Hz}, 2 \mathrm{H}), 7.36(\mathrm{~d}, J=8.1 \mathrm{~Hz}, 2 \mathrm{H}), 4.25(\mathrm{q}, J=7.1 \mathrm{~Hz}, 2 \mathrm{H}), 3.94(\mathrm{q}, J=7.1 \mathrm{~Hz}, 2 \mathrm{H})$, 3.70-3.49 (m, 2H), $1.34(\mathrm{~d}, J=4.4 \mathrm{~Hz}, 3 \mathrm{H}), 1.30(\mathrm{t}, J=7.1 \mathrm{~Hz}, 3 \mathrm{H}), 0.99(\mathrm{t}, J=7.1 \mathrm{~Hz}, 3 \mathrm{H}) ;{ }^{13} \mathrm{C}$ NMR $\left(101 \mathrm{MHz} \mathrm{CDCl}_{3}\right) \delta: 168.1,167.7,147.4,129.0\left(\mathrm{C}-\mathrm{F},{ }^{2} J_{\mathrm{C}-\mathrm{F}}=32.3 \mathrm{~Hz}\right), 127.9,125.4\left(\mathrm{C}-\mathrm{F},{ }^{3} J_{\mathrm{C}-\mathrm{F}}=4.0\right.$ $\mathrm{Hz}), 122.8\left(\mathrm{C}-\mathrm{F},{ }^{1} J_{\mathrm{C}-\mathrm{F}}=272.7 \mathrm{~Hz}\right), 61.7,61.3,58.9,39.8,20.1,14.1,13.7$; HRMS (ESI) m/z: [M+Na ${ }^{+}$ calcd for $\mathrm{C}_{16} \mathrm{H}_{19} \mathrm{~F}_{3} \mathrm{O}_{4} \mathrm{Na}$ 355.1128; Found 355.1133. HPLC analysis (Chiralpak AD-H column, hexane: isopropanol $=95: 5$; flow rate $=1 \mathrm{~mL} / \mathrm{min}$; UV detection at $210 \mathrm{~nm}): t_{\mathrm{R}}(R)=8.2$ min (major); $t_{\mathrm{R}}(S)=$ $8.9 \min$ (minor).

\section{(-)-Diethyl 2-(1-(4-(ethoxycarbonyl)phenyl)ethyl)malonate (3h)}<smiles>CCOC(=O)c1ccc(C(C)=C(C)C(C(=O)OCC)C(=O)OCC)cc1</smiles>

Colorless oil, 22 h, $326 \mathrm{mg}, 97 \%$ yield, $R_{\mathrm{f}}=0.5$ (petroleum ether/ethyl acetate $=5: 1), 87 \%$ ee. $[\alpha]_{\mathrm{D}}^{28}-14.8\left(c 1.0, \mathrm{CHCl}_{3}\right) ;{ }^{1} \mathrm{H}$ NMR $\left(400 \mathrm{MHz}, \mathrm{CDCl}_{3}\right) \delta$ : $7.89(\mathrm{~d}, J=8.4 \mathrm{~Hz}, 2 \mathrm{H}), 7.23(\mathrm{~d}, J=8.4 \mathrm{~Hz}, 2 \mathrm{H}), 4.28(\mathrm{q}, J=7.2 \mathrm{~Hz}, 2 \mathrm{H})$, $4.16(\mathrm{q}, J=7.2 \mathrm{~Hz}, 2 \mathrm{H}), 3.91-3.78(\mathrm{~m}, 2 \mathrm{H}), 3.58-3.46(\mathrm{~m}, 2 \mathrm{H}), 1.30(\mathrm{t}, J=$ $7.2 \mathrm{~Hz}, 3 \mathrm{H}), 1.25(\mathrm{~d}, J=6.0 \mathrm{~Hz}, 3 \mathrm{H}), 1.21(\mathrm{t}, J=7.2 \mathrm{~Hz}, 3 \mathrm{H}), 0.92(\mathrm{t}, J=7.2 \mathrm{~Hz}, 3 \mathrm{H}) ;{ }^{13} \mathrm{C} \mathrm{NMR}(101$ $\left.\mathrm{MHz}_{\mathrm{CDCl}}\right) \delta$ : 168.1, 167.6, 166.4, 148.5, 129.7, 129.1, 127.5, 61.6, 61.2, 60.8, 58.8, 39.9, 20.0, 14.3, 14.1, 13.8; HRMS (ESI) m/z: [M+Na] ${ }^{+}$calcd for $\mathrm{C}_{18} \mathrm{H}_{24} \mathrm{O}_{6} \mathrm{Na} 359.1465$; Found 359.1470. HPLC analysis (Chiralpak AD-H column, hexane: isopropanol = 95:5; flow rate $=1 \mathrm{~mL} / \mathrm{min}$; UV detection at $210 \mathrm{~nm}$ ): $t_{\mathrm{R}}($ major $)=10.9 \mathrm{~min} ; t_{\mathrm{R}}($ minor $)=13.5 \mathrm{~min}$.

\section{(-)-(R)-Diethyl-2-(1-(4-phenylphenyl)ethyl)malonate $(3 i)^{16}$}

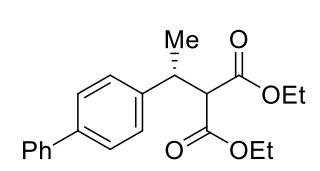

Colorless oil, $22 \mathrm{~h}, 326 \mathrm{mg}, 96 \%$ yield, $R_{\mathrm{f}}=0.7$ (petroleum ether/ethyl acetate $=$ $5: 1), 92 \%$ ee. $[\alpha]_{\mathrm{D}}^{23}-22.0\left(c 1.0, \mathrm{CHCl}_{3}\right)$ [the absolute configuration was determined by converting it to 3-([1,1'-biphenyl]-4-yl)butanoic acid and comparing its optical rotation that reported for $(S)$-isomer, $[\alpha]_{\mathrm{D}}^{26}-36.5(c$ 1.0, $\left.\mathrm{CH}_{3} \mathrm{OH}\right)$; lit. ${ }^{17}[\alpha]_{\mathrm{D}}^{20}+33.6\left(c 1.0, \mathrm{CH}_{3} \mathrm{OH}\right), 87 \%$ ee for $(S)$-isomer]. ${ }^{1} \mathrm{H} \mathrm{NMR}\left(400 \mathrm{MHz}, \mathrm{CDCl}_{3}\right) \delta: 7.59-$ $7.49(\mathrm{~m}, 4 \mathrm{H}), 7.44-7.38(\mathrm{~m}, 2 \mathrm{H}), 7.36-7.27(\mathrm{~m}, 3 \mathrm{H}), 4.25(\mathrm{q}, J=7.2 \mathrm{~Hz}, 2 \mathrm{H}), 3.94(\mathrm{q}, J=7.2 \mathrm{~Hz}, 2 \mathrm{H})$, $3.64(\mathrm{~d}, J=10.8 \mathrm{~Hz}, 1 \mathrm{H}), 3.61-3.53(\mathrm{~m}, 1 \mathrm{H}), 1.36(\mathrm{~d}, J=6.4 \mathrm{~Hz}, 3 \mathrm{H}), 1.30(\mathrm{t}, J=7.2 \mathrm{~Hz}, 3 \mathrm{H}), 0.98(\mathrm{t}$, $J=7.2 \mathrm{~Hz}, 3 \mathrm{H}$ ). HPLC analysis (Chiralpak AD-H column, hexane: isopropanol = 98:2; flow rate = 1 $\mathrm{mL} / \mathrm{min}$; UV detection at $210 \mathrm{~nm}$ ): $t_{\mathrm{R}}(R)=7.1 \mathrm{~min}$ (major); $t_{\mathrm{R}}(S)=9.4 \min$ (minor).

\section{(-)-Diethyl-2-(1-(p-tolyl)ethyl)malonate $(3 \mathbf{j})^{18}$}

$$
\begin{aligned}
& \text { Colorless oil, } 22 \mathrm{~h}, 267 \mathrm{mg}, 96 \% \text { yield, } R_{\mathrm{f}}=0.6 \text { (petroleum ether/ethyl acetate } \\
& =5: 1), 92 \% \text { ee. }[\alpha]_{\mathrm{D}}^{25}-17.2\left(c 1.0, \mathrm{CHCl}_{3}\right) .{ }^{1} \mathrm{H} \mathrm{NMR}_{\left(400 \mathrm{MHz}, \mathrm{CDCl}_{3}\right) \delta: 7.11}
\end{aligned}
$$


1.35-1.23 (m, 6H), $0.99(\mathrm{t}, J=7.2 \mathrm{~Hz}, 3 \mathrm{H})$. HPLC analysis (Chiralpak AD-H column, hexane: isopropanol $=90: 10$; flow rate $=1 \mathrm{~mL} / \mathrm{min} ; \mathrm{UV}$ detection at $210 \mathrm{~nm}): t_{\mathrm{R}}($ major $)=5.1 \mathrm{~min} ; t_{\mathrm{R}}($ minor $)=$ $5.8 \mathrm{~min}$.

\section{(-)-(R)-Diethyl-2-(1-(4-methoxyphenyl)ethyl)malonate (3k)}

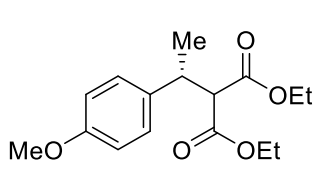

Colorless oil, 22 h, $276 \mathrm{mg}, 94 \%$ yield, $R_{\mathrm{f}}=0.6$ (petroleum ether/ethyl acetate $=5: 1), 90 \%$ ee. $[\alpha]_{\mathrm{D}}^{27}-17.2\left(c 0.5, \mathrm{CHCl}_{3}\right)$ [the absolute configuration was determined by converting it to ethyl 3-(4-methoxyphenyl)butanoate and comparing its optical rotation that reported for $(S)$-isomer, $[\alpha]_{\mathrm{D}}^{25}-26.1(c 0.59$,

$\left.\mathrm{CHCl}_{3}\right)$; lit. ${ }^{15}[\alpha]_{\mathrm{D}}^{23}+26.2\left(c 0.59, \mathrm{CHCl}_{3}\right), 92 \%$ ee for $(S)$-isomer]. ${ }^{1} \mathrm{H}$ NMR $\left(400 \mathrm{MHz}, \mathrm{CDCl}_{3}\right) \delta: 7.15$ $(\mathrm{d}, J=8.0 \mathrm{~Hz}, 2 \mathrm{H}), 6.82(\mathrm{~d}, J=8.0 \mathrm{~Hz}, 2 \mathrm{H}), 4.23(\mathrm{q}, J=7.2 \mathrm{~Hz}, 2 \mathrm{H}), 3.93(\mathrm{q}, J=7.2 \mathrm{~Hz}, 2 \mathrm{H}), 3.77$ (s, $3 \mathrm{H}), 3.54(\mathrm{~d}, J=10.8 \mathrm{~Hz}, 1 \mathrm{H}), 3.53-3.39(\mathrm{~m}, 1 \mathrm{H}), 1.35-1.22(\mathrm{~m}, 6 \mathrm{H}), 1.01(\mathrm{t}, J=7.2 \mathrm{~Hz}, 3 \mathrm{H}) ;{ }^{13} \mathrm{C} \mathrm{NMR}$ $\left(101 \mathrm{MHz}, \mathrm{CDCl}_{3}\right) \delta: 167.5,167.0,157.4,134.2,127.4,112.7,60.4,60.1,58.6,54.2,38.3,19.3,13.1$, 12.8; HRMS (ESI) m/z: [M+Na] $]^{+}$calcd for $\mathrm{C}_{16} \mathrm{H}_{22} \mathrm{O}_{5} \mathrm{Na}$ 317.1359; Found 317.1365. HPLC analysis (Chiralpak AD-H column, hexane: isopropanol = 90:10; flow rate $=1 \mathrm{~mL} / \mathrm{min}$; UV detection at $210 \mathrm{~nm}$ ): $t_{\mathrm{R}}(R)=7.2 \min$ (major); $t_{\mathrm{R}}(S)=9.2 \min$ (major).

\section{(-)-Diethyl-2-(1-(3-fluorophenyl)ethyl)malonate (3l)}

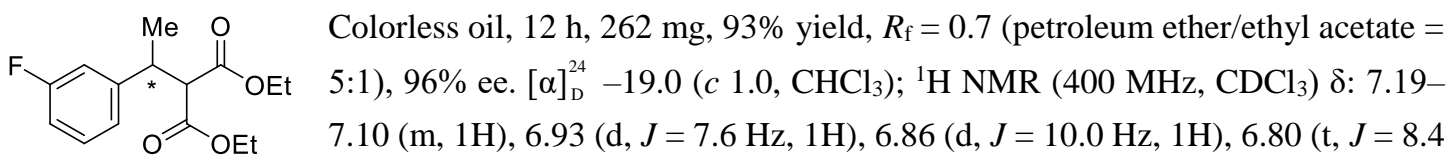
$\mathrm{Hz}, 1 \mathrm{H}), 4.14$ (q, $J=7.2 \mathrm{~Hz}, 2 \mathrm{H}), 3.86(\mathrm{q}, J=7.2 \mathrm{~Hz}, 2 \mathrm{H}), 3.50(\mathrm{~d}, J=10.8 \mathrm{~Hz}, 1 \mathrm{H}), 3.48-3.38(\mathrm{~m}, 1 \mathrm{H})$, $1.29-1.13(\mathrm{~m}, 6 \mathrm{H}), 0.91(\mathrm{t}, J=7.2 \mathrm{~Hz}, 3 \mathrm{H}) ;{ }^{13} \mathrm{C} \mathrm{NMR}\left(101 \mathrm{MHz}, \mathrm{CDCl}_{3}\right) \delta: 168.1,167.7,162.7(\mathrm{C}-\mathrm{F}$, $\left.{ }^{1} J_{\mathrm{C}-\mathrm{F}}=247.5 \mathrm{~Hz}\right), 145.8\left(\mathrm{C}-\mathrm{F},{ }^{3} J_{\mathrm{C}-\mathrm{F}}=7.1 \mathrm{~Hz}\right), 129.8\left(\mathrm{C}-\mathrm{F},{ }^{3} J_{\mathrm{C}-\mathrm{F}}=9.1 \mathrm{~Hz}\right), 123.2\left(\mathrm{C}-\mathrm{F},{ }^{4} J_{\mathrm{C}-\mathrm{F}}=2.0 \mathrm{~Hz}\right)$, $114.4\left(\mathrm{C}-\mathrm{F},{ }^{2} J_{\mathrm{C}-\mathrm{F}}=21.2 \mathrm{~Hz}\right), 113.7\left(\mathrm{C}-\mathrm{F},{ }^{2} J_{\mathrm{C}-\mathrm{F}}=21.2 \mathrm{~Hz}\right), 61.5,61.2,59.0,39.7,20.0,14.0,13.7$; HRMS (ESI) $\mathrm{m} / \mathrm{z}$ : $[\mathrm{M}+\mathrm{Na}]^{+}$calcd for $\mathrm{C}_{15} \mathrm{H}_{19} \mathrm{FO}_{4} \mathrm{Na} 305.1160$; Found 305.1164. HPLC analysis (Chiralpak AD$\mathrm{H}$ column, hexane: isopropanol $=98: 2$; flow rate $=1 \mathrm{~mL} / \mathrm{min}$; UV detection at $210 \mathrm{~nm}): t_{\mathrm{R}}($ major $)=9.4$ $\min ; t_{\mathrm{R}}($ minor $)=9.9 \min$.

\section{(-)-Diethyl-2-(1-(m-tolyl)ethyl)malonate (3m)}<smiles>CCOC(=O)C(C(=O)OCC)C(C)c1cccc(C)c1</smiles>

Colorless oil, $36 \mathrm{~h}, 264 \mathrm{mg}, 95 \%$ yield $(\mathrm{S} / \mathrm{C}=500), R_{\mathrm{f}}=0.6$ (petroleum ether/ethyl acetate $=5: 1), 94 \%$ ee. $[\alpha]_{\mathrm{D}}^{28}-20.4\left(c 1.0, \mathrm{CHCl}_{3}\right) ;{ }^{1} \mathrm{H}$ NMR $(400$ $\left.\mathrm{MHz} \mathrm{CDCl}_{3}\right) \delta: 7.09(\mathrm{t}, J=7.2 \mathrm{~Hz}, 1 \mathrm{H}), 7.00-6.89(\mathrm{~m}, 3 \mathrm{H}), 4.16(\mathrm{q}, J=7.2$ $\mathrm{Hz}, 2 \mathrm{H}), 3.86(\mathrm{q}, J=7.2 \mathrm{~Hz}, 2 \mathrm{H}), 3.52(\mathrm{~d}, J=10.8 \mathrm{~Hz}, 1 \mathrm{H}), 3.47-3.35(\mathrm{~m}, 1 \mathrm{H})$, $2.24(\mathrm{~s}, 3 \mathrm{H}), 1.24(\mathrm{~d}, J=6.8 \mathrm{~Hz}, 3 \mathrm{H}), 1.22(\mathrm{q}, J=7.2 \mathrm{~Hz}, 2 \mathrm{H}), 0.91(\mathrm{t}, J=7.2 \mathrm{~Hz}, 3 \mathrm{H}) ;{ }^{13} \mathrm{C} \mathrm{NMR}(101$ $\mathrm{MHz}_{\mathrm{CDCl}}$ ) $\delta:$ 168.5, 168.0, 143.1, 137.9, 128.3, 128.3, 127.5, 124.4, 61.4, 61.1, 59.3, 40.0, 21.4, 20.3, 14.1, 13.8; HRMS (ESI) m/z: [M+Na] ${ }^{+}$calcd for $\mathrm{C}_{16} \mathrm{H}_{22} \mathrm{O}_{4} \mathrm{Na} 301.1410$; Found 301.1415. HPLC analysis (Chiralpak IH-3 column, hexane: isopropanol = 99:1; flow rate $=1 \mathrm{~mL} / \mathrm{min}$; UV detection at $210 \mathrm{~nm}$ ): $t_{\mathrm{R}}($ major $)=4.6 \mathrm{~min} ; t_{\mathrm{R}}($ minor $)=4.8 \mathrm{~min}$.

\section{(-)-Diethyl-2-(1-(3-methoxyphenyl)ethyl)malonate (3n)}<smiles>CCOC(=O)C(C(=O)OCC)/C(C)=C(\C)c1cccc(OC)c1</smiles>

Colorless oil, $36 \mathrm{~h}, 279 \mathrm{mg}$, 95\% yield $(\mathrm{S} / \mathrm{C}=500), R_{\mathrm{f}}=0.6$ (petroleum ether/ethyl acetate $=5: 1), 95 \%$ ee. $[\alpha]_{\mathrm{D}}^{26}-18.8\left(c 1.0, \mathrm{CHCl}_{3}\right) ;{ }^{1} \mathrm{H}$ NMR $(400$ $\left.\mathrm{MHz} \mathrm{CDCl}_{3}\right) \delta: 7.11(\mathrm{t}, J=8.0 \mathrm{~Hz}, 1 \mathrm{H}), 6.94(\mathrm{~d}, J=8.0 \mathrm{~Hz}, 1 \mathrm{H}), 6.73-6.63$ 
(m, 2H), 4.20-4.11 (m, 2H), $3.86(\mathrm{q}, J=7.2 \mathrm{~Hz}, 2 \mathrm{H}), 3.70(\mathrm{~s}, 3 \mathrm{H}), 3.52(\mathrm{~d}, J=10.8 \mathrm{~Hz}, 1 \mathrm{H}), 3.48-3.37$ $(\mathrm{m}, 1 \mathrm{H}), 1.25-1.17(\mathrm{~m}, 6 \mathrm{H}), 0.92(\mathrm{t}, J=7.2 \mathrm{~Hz}, 3 \mathrm{H}) ;{ }^{13} \mathrm{C} \mathrm{NMR}\left(101 \mathrm{MHz}, \mathrm{CDCl}_{3}\right) \delta: 168.4,167.9,159.6$, 144.8, 129.4, 119.7, 113.3, 112.1, 61.5, 61.1, 59.2, 55.1, 40.1, 20.2, 14.1, 13.8; HRMS (ESI) m/z: $[\mathrm{M}+\mathrm{Na}]^{+}$calcd for $\mathrm{C}_{16} \mathrm{H}_{22} \mathrm{O}_{5} \mathrm{Na}$ 317.1359; Found 317.1364. HPLC analysis (Chiralpak IH-3 column, hexane: isopropanol $=95: 5$; flow rate $=1 \mathrm{~mL} / \mathrm{min}$; $\mathrm{UV}$ detection at $210 \mathrm{~nm}): t_{\mathrm{R}}($ major $)=5.2 \mathrm{~min} ; t_{\mathrm{R}}$ $($ minor $)=5.4 \mathrm{~min}$.

\section{(-)-Diethyl-2-(1-(2-fluorophenyl)ethyl)malonate (3o)}

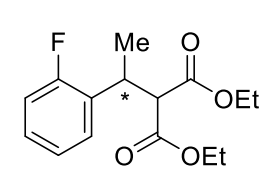

Colorless oil, $12 \mathrm{~h}, 270 \mathrm{mg}, 96 \%$ yield, $R_{\mathrm{f}}=0.7$ (petroleum ether/ethyl acetate $=5: 1$ ), $99 \%$ ee. $[\alpha]_{\mathrm{D}}^{27}-16.2\left(c 1.0, \mathrm{CHCl}_{3}\right) ;{ }^{1} \mathrm{H} \mathrm{NMR}\left(400 \mathrm{MHz}, \mathrm{CDCl}_{3}\right) \delta: 7.27-7.13(\mathrm{~m}$, $2 \mathrm{H}), 7.05(\mathrm{t}, J=7.6 \mathrm{~Hz}, 1 \mathrm{H}), 7.04-6.93(\mathrm{t}, J=9.4 \mathrm{~Hz}, 1 \mathrm{H}), 4.22(\mathrm{qd}, J=7.2,1.4 \mathrm{~Hz}$, $2 \mathrm{H}), 3.93(\mathrm{q}, J=7.2 \mathrm{~Hz}, 2 \mathrm{H}), 3.85-3.71(\mathrm{~m}, 2 \mathrm{H}), 1.35(\mathrm{~d}, J=5.2 \mathrm{~Hz}, 3 \mathrm{H}), 1.27(\mathrm{t}, J$ $=7.2 \mathrm{~Hz}, 3 \mathrm{H}), 0.97(\mathrm{t}, J=7.2 \mathrm{~Hz}, 3 \mathrm{H}) ;{ }^{13} \mathrm{C} \mathrm{NMR}\left(101 \mathrm{MHz}, \mathrm{CDCl}_{3}\right) \delta: 168.2,167.8,160.8\left(\mathrm{C}-\mathrm{F},{ }^{1} J_{\mathrm{C}-\mathrm{F}}\right.$ $=246.4 \mathrm{~Hz}), 129.8\left(\mathrm{C}-\mathrm{F},{ }^{2} J_{\mathrm{C}-\mathrm{F}}=14.1 \mathrm{~Hz}\right), 129.3\left(\mathrm{C}-\mathrm{F},{ }^{3} J_{\mathrm{C}-\mathrm{F}}=4.0 \mathrm{~Hz}\right), 128.3\left(\mathrm{C}-\mathrm{F},{ }^{3} J_{\mathrm{C}-\mathrm{F}}=8.1 \mathrm{~Hz}\right), 124.0$ $\left(\mathrm{C}-\mathrm{F},{ }^{4} J_{\mathrm{C}-\mathrm{F}}=4.0 \mathrm{~Hz}\right), 115.5\left(\mathrm{C}-\mathrm{F},{ }^{2} J_{\mathrm{C}-\mathrm{F}}=22.2 \mathrm{~Hz}\right), 61.4,61.0,57.3\left(\mathrm{C}-\mathrm{F},{ }^{3} J_{\mathrm{C}-\mathrm{F}}=2.0 \mathrm{~Hz}\right), 34.7,18.7,14.0$, 13.6; HRMS (ESI) m/z: [M+Na] ${ }^{+}$calcd for $\mathrm{C}_{15} \mathrm{H}_{19} \mathrm{FO}_{4} \mathrm{Na}$ 305.1160; Found 305.1165. HPLC analysis (Chiralpak AD-H column, hexane: isopropanol = 95:5; flow rate $=1 \mathrm{~mL} / \mathrm{min}$; UV detection at $210 \mathrm{~nm}$ ): $t_{\mathrm{R}}($ major $)=6.8 \mathrm{~min} ; t_{\mathrm{R}}($ minor $)=7.8 \mathrm{~min}$.

\section{(-)-Diethyl-2-(1-(o-tolyl)ethyl)malonate (3p)}

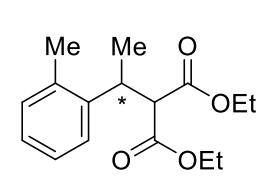

Colorless oil, 48 h, $250 \mathrm{mg}, 90 \%$ yield $(\mathrm{S} / \mathrm{C}=500), R_{\mathrm{f}}=0.8$ (petroleum ether/ethyl acetate $=5: 1), 99 \%$ ee. $[\alpha]_{\mathrm{D}}^{26}-1.6\left(c 1.0, \mathrm{CHCl}_{3}\right) ;{ }^{1} \mathrm{H} \mathrm{NMR}\left(400 \mathrm{MHz}, \mathrm{CDCl}_{3}\right) \delta$ : $7.23-7.03(\mathrm{~m}, 4 \mathrm{H}), 4.25(\mathrm{q}, J=6.8 \mathrm{~Hz}, 2 \mathrm{H}), 3.91(\mathrm{q}, J=6.8 \mathrm{~Hz}, 2 \mathrm{H}), 3.86-3.77(\mathrm{~m}$, $1 \mathrm{H}), 3.71(\mathrm{dd}, J=11.0,1.9 \mathrm{~Hz}, 1 \mathrm{H}), 2.41(\mathrm{~s}, 3 \mathrm{H}), 1.30(\mathrm{t}, J=6.8 \mathrm{~Hz}, 3 \mathrm{H}), 1.24(\mathrm{~d}, J$ $=6.8 \mathrm{~Hz}, 3 \mathrm{H}), 0.94(\mathrm{t}, J=6.8 \mathrm{~Hz}, 3 \mathrm{H}) ;{ }^{13} \mathrm{C} \mathrm{NMR}\left(101 \mathrm{MHz}, \mathrm{CDCl}_{3}\right) \delta: 168.7,168.1,141.7,135.8,130.5$, 126.4, 126.1, 125.7, 61.5, 61.1, 58.6, 34.8, 20.2, 19.7, 14.1, 13.7; HRMS (ESI) m/z: [M+Na] ${ }^{+}$calcd for $\mathrm{C}_{16} \mathrm{H}_{22} \mathrm{O}_{4} \mathrm{Na}$ 301.1410; Found 301.1415. HPLC analysis (Chiralpak AD-H column, hexane: isopropanol $=95: 5$; flow rate $=1 \mathrm{~mL} / \mathrm{min}$; UV detection at $210 \mathrm{~nm}): t_{\mathrm{R}}($ major $)=5.9 \mathrm{~min} ; t_{\mathrm{R}}($ minor $)=8.2 \mathrm{~min}$.

\section{(-)-(R)-Diethyl-2-(1-(benzo[d][1,3]dioxol-5-yl)ethyl)malonate (3q)}

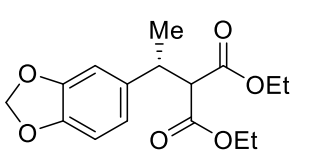

Colorless oil, $24 \mathrm{~h}, 286 \mathrm{mg}, 93 \%$ yield, $R_{\mathrm{f}}=0.4$ (petroleum ether/ethyl acetate $=$ $5: 1), 91 \%$ ee. $[\alpha]_{\mathrm{D}}^{24}-28.2\left(c 1.0, \mathrm{CHCl}_{3}\right)$ [the absolute configuration was determined by converting it to ethyl 3-(benzo[ $d][1,3]$ dioxol-5-yl)butanoate and comparing its optical rotation that reported for $(S)$-isomer, $[\alpha]_{\mathrm{D}}^{25}-34.0\left(c 0.63, \mathrm{CHCl}_{3}\right) ;$ lit. $^{15}[\alpha]_{\mathrm{D}}^{23}+33.6$ (c $\left.0.63, \mathrm{CHCl}_{3}\right), 89 \%$ ee for $(S)$-isomer]. ${ }^{1} \mathrm{H} \mathrm{NMR}\left(400 \mathrm{MHz}, \mathrm{CDCl}_{3}\right) \delta$ : 6.75-6.66 (m, 3H), $5.89(\mathrm{~s}, 2 \mathrm{H})$, $4.22(\mathrm{q}, J=7.1 \mathrm{~Hz}, 2 \mathrm{H}), 3.96(\mathrm{q}, J=7.1 \mathrm{~Hz}, 2 \mathrm{H}), 3.53$ (d, $J=10.6 \mathrm{~Hz}, 1 \mathrm{H}), 3.49-3.40(\mathrm{~m}, 1 \mathrm{H}), 1.31-$ $1.22(\mathrm{~m}, 6 \mathrm{H}), 1.04(\mathrm{t}, J=7.1 \mathrm{~Hz}, 3 \mathrm{H}) ;{ }^{13} \mathrm{C} \mathrm{NMR}\left(101 \mathrm{MHz}, \mathrm{CDCl}_{3}\right) \delta: 168.2,167.8,147.5,146.2,137.0$, 120.6, 108.0, 107.7, 100.8, 61.3, 61.0, 59.4, 39.7, 20.2, 14.0, 13.8; HRMS (ESI) m/z: [M+Na] ${ }^{+}$calcd for $\mathrm{C}_{16} \mathrm{H}_{20} \mathrm{O}_{6} \mathrm{Na} 331.1152$; Found 331.1156. HPLC analysis (Chiralpak IH-3 column, hexane: isopropanol $=95: 5$; flow rate $=1 \mathrm{~mL} / \mathrm{min} ; \mathrm{UV}$ detection at $210 \mathrm{~nm}): t_{\mathrm{R}}(R)=7.6 \mathrm{~min}($ major $) ; t_{\mathrm{R}}(S)=9.0 \mathrm{~min}($ minor $)$.

(+)-Diethyl-2-(1-(naphthalen-1-yl)ethyl)malonate (3r) 


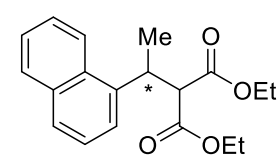

Colorless oil, 36 h, $305 \mathrm{mg}, 97 \%$ yield $(\mathrm{S} / \mathrm{C}=500), R_{\mathrm{f}}=0.7$ (petroleum ether/ethyl acetate $=5: 1), 98 \%$ ee. $[\alpha]_{\mathrm{D}}^{26}+46.8\left(c 1.0, \mathrm{CHCl}_{3}\right) ;{ }^{1} \mathrm{H} \mathrm{NMR}\left(400 \mathrm{MHz}, \mathrm{CDCl}_{3}\right) \delta$ : $8.24(\mathrm{~d}, J=8.4 \mathrm{~Hz}, 1 \mathrm{H}), 7.82(\mathrm{~d}, J=7.6 \mathrm{~Hz}, 1 \mathrm{H}), 7.70(\mathrm{~d}, J=7.6 \mathrm{~Hz}, 1 \mathrm{H}), 7.55-$ $7.50(\mathrm{~m}, 1 \mathrm{H}), 7.49-7.35(\mathrm{~m}, 3 \mathrm{H}), 4.53-4.44(\mathrm{~m}, 1 \mathrm{H}), 4.24(\mathrm{q}, J=7.2 \mathrm{~Hz}, 2 \mathrm{H}), 3.91$ $(\mathrm{d}, J=10.4 \mathrm{~Hz}, 1 \mathrm{H}), 3.85-3.75(\mathrm{~m}, 2 \mathrm{H}), 1.43(\mathrm{~d}, J=6.8 \mathrm{~Hz}, 3 \mathrm{H}), 1.28(\mathrm{t}, J=7.2 \mathrm{~Hz}, 3 \mathrm{H}), 0.78(\mathrm{t}, J=$ $7.2 \mathrm{~Hz}, 3 \mathrm{H}) ;{ }^{13} \mathrm{C} \mathrm{NMR}\left(101 \mathrm{MHz}, \mathrm{CDCl}_{3}\right) \delta: 168.7,168.1,139.7,134.0,131.3,128.9,127.3,126.1$, 125.6, 125.4, 123.4, 123.2, 61.6, 61.2, 58.7, 34.0, 20.3, 14.2, 13.6; HRMS (ESI) m/z: [M+Na $]^{+}$calcd for $\mathrm{C}_{19} \mathrm{H}_{22} \mathrm{O}_{4} \mathrm{Na}$ 337.1410; Found 337.1415. HPLC analysis (Chiralpak IH-3 column, hexane: isopropanol $=95: 5$; flow rate $=1 \mathrm{~mL} / \mathrm{min} ; \mathrm{UV}$ detection at $210 \mathrm{~nm}): t_{\mathrm{R}}($ major $)=5.2 \mathrm{~min} ; t_{\mathrm{R}}($ minor $)=5.4 \mathrm{~min}$.

\section{(-)-Diethyl-2-(1-(naphthalen-2-yl)ethyl)malonate (3s)}

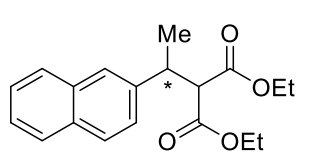

Colorless oil, $20 \mathrm{~h}, 292 \mathrm{mg}, 93 \%$ yield, $R_{\mathrm{f}}=0.7$ (petroleum ether/ethyl acetate $=$ 5:1), $92 \%$ ee. $[\alpha]_{\mathrm{D}}^{27}-16.6\left(c \quad 1.0, \mathrm{CHCl}_{3}\right) ;{ }^{1} \mathrm{H} \mathrm{NMR}\left(400 \mathrm{MHz}, \mathrm{CDCl}_{3}\right) \delta$ : $7.82-7.76(\mathrm{~m}, 3 \mathrm{H}), 7.67(\mathrm{~s}, 1 \mathrm{H}), 7.48-7.40(\mathrm{~m}, 2 \mathrm{H}), 7.38(\mathrm{dd}, J=8.4,1.6 \mathrm{~Hz}$, $1 \mathrm{H}), 4.25(\mathrm{q}, J=7.2 \mathrm{~Hz}, 2 \mathrm{H}), 3.94-3.82(\mathrm{~m}, 2 \mathrm{H}), 3.77-3.65(\mathrm{~m}, 2 \mathrm{H}), 1.41(\mathrm{~d}, J$ $=6.0 \mathrm{~Hz}, 3 \mathrm{H}), 1.30(\mathrm{t}, J=7.2 \mathrm{~Hz}, 3 \mathrm{H}), 0.89(\mathrm{t}, J=7.2 \mathrm{~Hz}, 3 \mathrm{H}) ;{ }^{13} \mathrm{C} \mathrm{NMR}\left(101 \mathrm{MHz}, \mathrm{CDCl}_{3}\right) \delta: 168.5$, 168.0, 140.7, 133.4, 132.5, 128.1, 127.7, 127.6, 126.1, 126.0, 125.7, 125.6, 61.6, 61.2, 59.3, 40.2, 20.3, 14.2, 13.7; HRMS (ESI) m/z: [M+Na] ${ }^{+}$calcd for $\mathrm{C}_{19} \mathrm{H}_{22} \mathrm{O}_{4} \mathrm{Na} 337.1410$; Found 337.1414. HPLC analysis (Chiralpak AD-H column, hexane: isopropanol = 98:2; flow rate $=1 \mathrm{~mL} / \mathrm{min}$; UV detection at $210 \mathrm{~nm}$; $t_{\mathrm{R}}($ major $)=19.2 \mathrm{~min} ; t_{\mathrm{R}}($ minor $)=21.2 \mathrm{~min}$.

\section{(-)-Diethyl-2-(1-(thiophen-2-yl)ethyl)malonate (3t)}

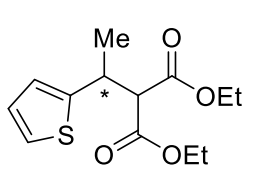

Colorless oil, $22 \mathrm{~h}, 248 \mathrm{mg}, 92 \%$ yield, $R_{\mathrm{f}}=0.7$ (petroleum ether/ethyl acetate $=5: 1$ ), $88 \%$ ee. $[\alpha]_{\mathrm{D}}^{25}-29.2\left(c 0.5, \mathrm{CHCl}_{3}\right) ;{ }^{1} \mathrm{H} \mathrm{NMR}\left(400 \mathrm{MHz}, \mathrm{CDCl}_{3}\right) \delta: 7.15(\mathrm{dd}, J=4.8$, $0.8 \mathrm{~Hz}, 1 \mathrm{H}), 6.90(\mathrm{dd}, J=4.8,3.2 \mathrm{~Hz}, 1 \mathrm{H}), 6.87(\mathrm{dd}, J=3.2,0.8 \mathrm{~Hz}, 1 \mathrm{H}), 4.22(\mathrm{q}, J$ $=7.2 \mathrm{~Hz}, 2 \mathrm{H}), 4.03(\mathrm{q}, J=7.2 \mathrm{~Hz}, 2 \mathrm{H}), 3.92-3.83(\mathrm{~m}, 1 \mathrm{H}), 3.57(\mathrm{~d}, J=9.6 \mathrm{~Hz}, 1 \mathrm{H})$, $1.42(\mathrm{~d}, J=6.8 \mathrm{~Hz}, 3 \mathrm{H}), 1.28(\mathrm{t}, J=7.2 \mathrm{~Hz}, 3 \mathrm{H}), 1.09(\mathrm{t}, J=7.2 \mathrm{~Hz}, 3 \mathrm{H}) ;{ }^{13} \mathrm{C} \mathrm{NMR}\left(101 \mathrm{MHz}, \mathrm{CDCl}_{3}\right)$

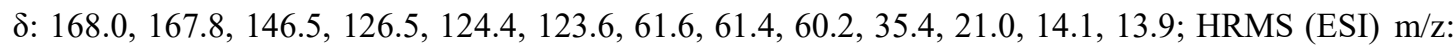
$[\mathrm{M}+\mathrm{Na}]^{+}$calcd for $\mathrm{C}_{13} \mathrm{H}_{18} \mathrm{O}_{4} \mathrm{SNa}$ 293.0818; Found 293.0824. HPLC analysis (Chiralpak AD-H column, hexane: isopropanol $=98: 2$; flow rate $=1 \mathrm{~mL} / \mathrm{min}$; $\mathrm{UV}$ detection at $230 \mathrm{~nm}$ ): $t_{\mathrm{R}}$ (major) $=7.8 \mathrm{~min} ; t_{\mathrm{R}}$ $($ minor $)=8.3 \mathrm{~min}$.

\section{(-)-Diethyl-2-(1-(furan-2-yl)ethyl)malonate (3u) ${ }^{19}$}

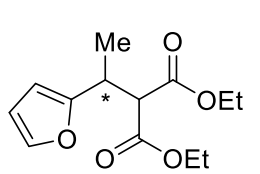

Colorless oil, 48 h, $229 \mathrm{mg}, 90 \%$ yield, $R_{\mathrm{f}}=0.6$ (petroleum ether/ethyl acetate $=5: 1$ ), $52 \%$ ee. $[\alpha]_{\mathrm{D}}^{27}-6.0\left(c 1.0, \mathrm{CHCl}_{3}\right) .{ }^{1} \mathrm{H}$ NMR $\left(400 \mathrm{MHz}, \mathrm{CDCl}_{3}\right) \delta: 7.30(\mathrm{~d}, J=1.0$ $\mathrm{Hz}, 1 \mathrm{H}), 6.27-6.25(\mathrm{~m}, 1 \mathrm{H}), 6.06(\mathrm{~d}, J=3.1 \mathrm{~Hz}, 1 \mathrm{H}), 4.21(\mathrm{q}, J=7.1 \mathrm{~Hz}, 2 \mathrm{H}), 4.13-$ $4.04(\mathrm{~m}, 2 \mathrm{H}), 3.70-3.60(\mathrm{~m}, 2 \mathrm{H}), 1.37-1.32(\mathrm{~m}, 3 \mathrm{H}), 1.26(\mathrm{t}, J=7.1 \mathrm{~Hz}, 3 \mathrm{H}), 1.16$ (t, $J=7.1 \mathrm{~Hz}, 3 \mathrm{H}$ ). HPLC analysis (Chiralpak IC-3 column, hexane: isopropanol = 95:5; flow rate = 1 $\mathrm{mL} / \mathrm{min}$; UV detection at $210 \mathrm{~nm}): t_{\mathrm{R}}($ major $)=10.4 \mathrm{~min} ; t_{\mathrm{R}}($ minor $)=11.5 \mathrm{~min}$.

\section{(-)-Diethyl-2-(1-cyclohexylethyl)malonate (3v) ${ }^{20}$}

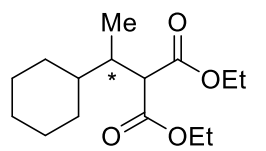

Colorless oil, 36 h, $227 \mathrm{mg}, 84 \%$ yield $(\mathrm{S} / \mathrm{C}=500), R_{\mathrm{f}}=0.7$ (petroleum ether/ethyl acetate $=5: 1), 58 \%$ ee. $[\alpha]_{\mathrm{D}}^{27}-1.2\left(c 1.0, \mathrm{CHCl}_{3}\right) .{ }^{1} \mathrm{H} \mathrm{NMR}\left(400 \mathrm{MHz}, \mathrm{CDCl}_{3}\right) \delta$ : $4.25-4.10(\mathrm{~m}, 4 \mathrm{H}), 3.37(\mathrm{~d}, J=8.8 \mathrm{~Hz}, 1 \mathrm{H}), 2.22-2.10(\mathrm{~m}, 1 \mathrm{H}), 1.79-1.71(\mathrm{~m}, 2 \mathrm{H})$, 
$1.69-1.55(\mathrm{~m}, 3 \mathrm{H}), 1.35-1.04(\mathrm{~m}, 11 \mathrm{H}), 0.96(\mathrm{td}, J=12.0,3.2 \mathrm{~Hz}, 1 \mathrm{H}), 0.91(\mathrm{~d}, J=7.2 \mathrm{~Hz}, 3 \mathrm{H})$. HPLC analysis (Chiralpak AD-H column, hexane: isopropanol $=90: 10$; flow rate $=1 \mathrm{~mL} / \mathrm{min}$; UV detection at $254 \mathrm{~nm}): t_{\mathrm{R}}($ major $)=6.0 \mathrm{~min} ; t_{\mathrm{R}}($ minor $)=6.7 \mathrm{~min}$ (Note: HPLC analysis was detected by transformation of it into 3-cyclohexyl- $N$-phenylbutanamide).

Asymmetric hydrogenation of 2 a at $\mathbf{S} / \mathbf{C}=\mathbf{1 0 , 0 0 0}$ : To a $60 \mathrm{~mL}$ hydrogenation vessel containing $\alpha, \beta$ unsaturated malonate $\mathbf{2 a}(2.62 \mathrm{~g}, 10.0 \mathrm{mmol})$ was added a solution of iridium catalyst $(R)-\mathbf{1} \mathbf{m}(1.3 \mathrm{mg}$, $0.001 \mathrm{mmol})$ in EtOH $(1.0 \mathrm{~mL})$ and a solution of $t \mathrm{BuOK}(224 \mathrm{mg}, 2.0 \mathrm{mmol})$ in $\mathrm{EtOH}(9.0 \mathrm{~mL})$ under nitrogen atmosphere. The autoclave was purged with hydrogen by pressurizing to $5 \mathrm{~atm}$ and releasing the pressure. This procedure was repeated three times and then pressurized to $80 \mathrm{~atm}$ of $\mathrm{H}_{2}$. The reaction mixture was stirred at room temperature $\left(25-30^{\circ} \mathrm{C}\right)$ for $66 \mathrm{~h}$. After releasing the hydrogen pressure, the reaction mixtures were concentrated in vacuo, and purified by flash chromatography on silica gel with petroleum ether/ethyl acetate $(5: 1)$ as an eluent to provide $(R)-3 \mathbf{a}$ in $96 \%$ yield $(2.59 \mathrm{~g}, 98 \%$ conversion) with $93 \%$ ee $(R)$.

Asymmetric hydrogenation of 2 a at $\mathbf{S} / \mathbf{C}=\mathbf{2 0 , 0 0 0}$ : To a $60 \mathrm{~mL}$ hydrogenation vessel containing $\alpha, \beta$ unsaturated malonate $\mathbf{2 a}(5.24 \mathrm{~g}, 20.0 \mathrm{mmol})$ was added a solution of iridium catalyst $(R)-\mathbf{1} \mathbf{m}(1.3 \mathrm{mg}$, $0.001 \mathrm{mmol})$ in $\mathrm{EtOH}(1.0 \mathrm{~mL})$ and a solution of $t \mathrm{BuOK}(448 \mathrm{mg}, 4.0 \mathrm{mmol})$ in $\mathrm{EtOH}(14.0 \mathrm{~mL})$ under nitrogen atmosphere. The autoclave was purged with hydrogen by pressurizing to $5 \mathrm{~atm}$ and releasing the pressure. This procedure was repeated three times and then pressurized to $80 \mathrm{~atm}$ of $\mathrm{H}_{2}$. The reaction mixture was stirred at room temperature $\left(25-30{ }^{\circ} \mathrm{C}\right)$ for $6 \mathrm{~d}$. After releasing the hydrogen pressure, the reaction mixtures were concentrated in vacuo, and purified by flash chromatography on silica gel with petroleum ether/ethyl acetate (5:1) as an eluent to provide $(R)-3 \mathbf{a}$ in $94 \%$ yield (4.96 g, 95\% conversion) with $94 \%$ ee.

\section{(D) Conversions of Hydrogenated Products}

\section{Conversions of Hydrogenated Product $(R)-3 a$.}<smiles>CCOC(=O)CC(C)c1ccccc1</smiles>

$(R)-4$

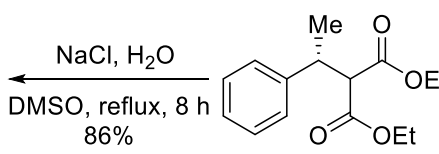

$(R)-3 \mathbf{a}$

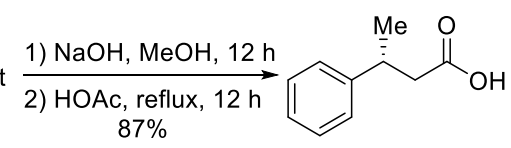

$(R)-5$

$$
\begin{array}{c|}
\mathrm{KOH}(\mathrm{aq}) \\
\mathrm{EtOH}, \mathrm{rt}, 5 \mathrm{~h}
\end{array}
$$

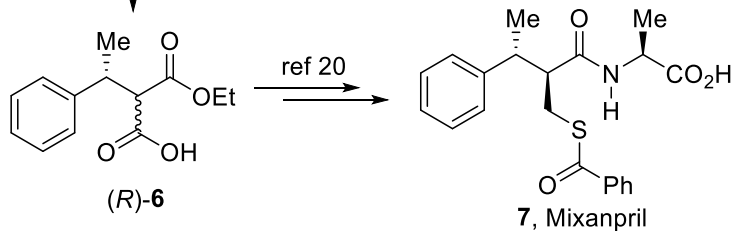

\section{Synthesis of $(R)$-ethyl 3-phenylbutanoate $((R)-4)^{10}$}

To a solution of $(R)-3 \mathbf{a}(1.32 \mathrm{~g}, 5 \mathrm{mmol})$ in DMSO $(15 \mathrm{~mL})$ was added $\mathrm{NaCl}(614 \mathrm{mg}, 10.5 \mathrm{mmol})$ and $\mathrm{H}_{2} \mathrm{O}(189 \mu \mathrm{L}, 10.5 \mathrm{mmol})$. The resulting reaction mixture was then heated to reflux in an oil bath for 8 h, cooled to room temperature, diluted with $\mathrm{H}_{2} \mathrm{O}(20 \mathrm{~mL})$ and extracted with ethyl acetate $(3 \times 20 \mathrm{~mL})$. The combined organic layers were washed with water $(20 \mathrm{~mL})$ and brine $(20 \mathrm{~mL})$, dried over $\mathrm{MgSO}_{4}$, and concentrated in vacuo. The residue was purified by flash chromatography on silica gel with 
petroleum ether/ethyl acetate (10:1) as an eluent to give $826 \mathrm{mg}(R)-4$ as a colorless oil. $86 \%$ yield. $[\alpha]_{\mathrm{D}}^{21}$ -24.2 (c 0.94, $\left.\mathrm{CHCl}_{3}\right)\left(\right.$ lit. ${ }^{10}[\alpha]_{\mathrm{D}}^{20}-23.5$ (c $\left.0.94, \mathrm{CHCl}_{3}\right), 91 \%$ ee for $(R)$-isomer). ${ }^{1} \mathrm{H} \mathrm{NMR}(400 \mathrm{MHz}$, $\left.\mathrm{CDCl}_{3}\right) \delta: 7.25(\mathrm{dd}, J=10.1,4.7 \mathrm{~Hz}, 2 \mathrm{H}), 7.22-7.12(\mathrm{~m}, 3 \mathrm{H}), 4.04(\mathrm{q}, J=7.1 \mathrm{~Hz}, 2 \mathrm{H}), 3.33-3.20(\mathrm{~m}$, $1 \mathrm{H}), 2.58(\mathrm{dd}, J=15.0,7.1 \mathrm{~Hz}, 1 \mathrm{H}), 2.51(\mathrm{dd}, J=15.0,8.1 \mathrm{~Hz}, 1 \mathrm{H}), 1.28(\mathrm{~d}, J=7.0 \mathrm{~Hz}, 3 \mathrm{H}), 1.14(\mathrm{t}, J$ $=7.1 \mathrm{~Hz}, 3 \mathrm{H})$.

\section{Synthesis of $(R)$-3-phenylbutanoic acid $((R)-5)^{21}$}

To a solution of $(R)-3 \mathbf{a}(1.32 \mathrm{~g}, 5 \mathrm{mmol})$ in $\mathrm{MeOH}(15 \mathrm{~mL})$ was added an aqueous $\mathrm{NaOH}$ solution (3.3N, $9 \mathrm{~mL}, 30 \mathrm{mmol}$ ), and the resulting mixture was then heated to reflux in an oil bath for $12 \mathrm{~h}$. The reaction mixture was then concentrated in vacuo, redissolved with $\mathrm{AcOH}(33 \mathrm{~mL})$, and stirred at reflux for $12 \mathrm{~h}$. The reaction mixture was then concentrated in vacuo again, treated with water $(25 \mathrm{~mL})$, extracted with ethyl acetate $(3 \times 20 \mathrm{~mL})$. The combined organic layers were dried over $\mathrm{MgSO}_{4}$, concentrated in vacuo, and purified by flash chromatography on silica gel with petroleum ether/ethyl acetate (5:1) as an eluent to give $713 \mathrm{mg}$ of $(R)-5$ as a colorless oil. $87 \%$ yield, $[\alpha]_{\mathrm{D}}^{26}-54.7\left(c 1.12, \mathrm{C}_{6} \mathrm{H}_{6}\right)\left(\right.$ lit. $^{21}[\alpha]_{\mathrm{D}}^{22}-54.2(c 1.12$, $\left.\mathrm{C}_{6} \mathrm{H}_{6}\right), 87 \%$ ee for $(R)$-isomer). ${ }^{1} \mathrm{H}$ NMR $\left(400 \mathrm{MHz}, \mathrm{CDCl}_{3}\right) \delta$ : $11.30(\mathrm{~s}, 1 \mathrm{H}), 7.33-7.26(\mathrm{~m}, 2 \mathrm{H}), 7.25-$ 7.17 (m, 3H), 3.32-3.21 (m, 1H), 2.67 (dd, $J=15.5,6.8 \mathrm{~Hz}, 1 \mathrm{H}), 2.57$ (dd, $J=15.5,8.3 \mathrm{~Hz}, 1 \mathrm{H}), 1.32$ (d, $J=7.0 \mathrm{~Hz}, 3 \mathrm{H})$.

\section{Synthesis of (3R)-2-(ethoxycarbonyl)-3-phenylbutanoic acid $((\boldsymbol{R})-6)^{22}$}

To a solution of $(R)-\mathbf{3 a}(1.32 \mathrm{~g}, 5 \mathrm{mmol})$ in a mixture solvent of EtOH $(70 \mathrm{~mL})$ and $\mathrm{H}_{2} \mathrm{O}(70 \mathrm{~mL})$ was added an aqueous $\mathrm{KOH}$ solution $(2 \mathrm{~N}, 8.0 \mathrm{~mL}, 16 \mathrm{mmol})$, and the resulting reaction mixture was stirred at room temperature for $5 \mathrm{~h}$. The mixture was then concentrated in vacuo, diluted $\mathrm{H}_{2} \mathrm{O}(50 \mathrm{~mL})$, and acidified to $\mathrm{pH}<2$ with an aqueous $\mathrm{HCl}$ solution $(6 \mathrm{~N}, 10.0 \mathrm{~mL}, 60 \mathrm{mmol})$ at $0{ }^{\circ} \mathrm{C}$. The aqueous solution was then extracted with ethyl acetate $(3 \times 20 \mathrm{~mL})$, dried over $\mathrm{MgSO}_{4}$, concentrated in vacuo, and purified by flash chromatography on silica gel with petroleum ether/ethyl acetate (5:1) as an eluent to provide $1.05 \mathrm{~g}$ of $(R)-6$ as a colorless oil. $89 \%$ yield. $[\alpha]_{\mathrm{D}}^{23}-25.4\left(c 1.0, \mathrm{CHCl}_{3}\right) .{ }^{1} \mathrm{H} \mathrm{NMR}\left(400 \mathrm{MHz}, \mathrm{CDCl}_{3}\right) \delta$ : 11.07 (s, $1 \mathrm{H}), 7.35-7.16(\mathrm{~m}, 5 \mathrm{H}), 4.07$ (dq, $J=128.1,7.1 \mathrm{~Hz}, 2 \mathrm{H}), 3.62(\mathrm{dd}, J=15.2,10.4 \mathrm{~Hz}, 1 \mathrm{H}), 3.50$ (ddt, $J=17.7,13.9,6.9 \mathrm{~Hz}, 1 \mathrm{H}), 1.39-1.29$ (m, 3H), 1.29-0.91 (m, 3H).

\section{Enantioselective Synthesis of the chiral intermediate $(R)-8$ for $(R)-\mathrm{RC}-33$}
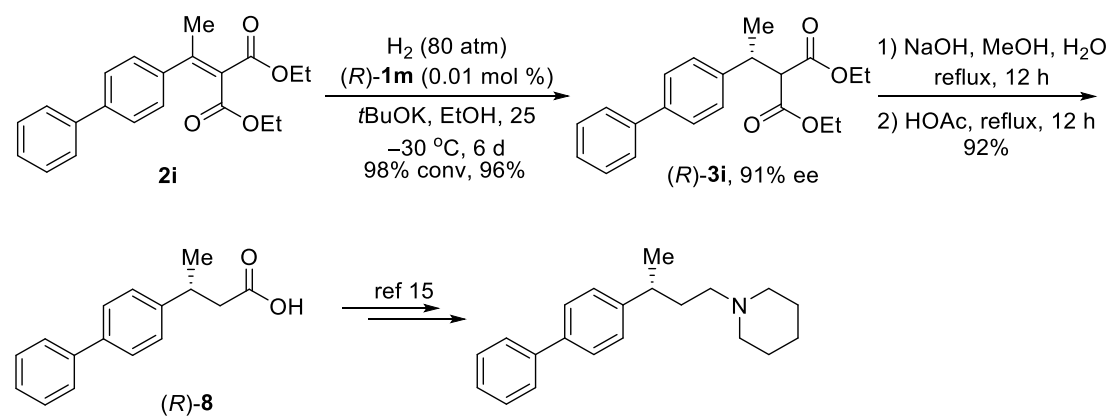

recrystal

$84 \%, 95 \%$ ee

(R)-RC-33

Synthesis of $(R)-3 i$ in gram-scale at $S / C=10,000$.

To a $60 \mathrm{~mL}$ hydrogenation vessel containing diethyl malonate $2 \mathbf{i}(3.38 \mathrm{~g}, 10.0 \mathrm{mmol})$ was added a solution of iridium catalyst $(R)-1 \mathrm{~m}$ in EtOH $(1.3 \mathrm{mg}, 0.001 \mathrm{mmol})$, and a solution of $t \mathrm{BuOK}(224 \mathrm{mg}$, $2.0 \mathrm{mmol})$ in EtOH $(9.0 \mathrm{~mL})$ under nitrogen atmosphere. The autoclave was purged with hydrogen by pressurizing to $5 \mathrm{~atm}$ and releasing the pressure. This procedure was repeated three times and then 
pressurized to $80 \mathrm{~atm}$ of $\mathrm{H}_{2}$. The reaction mixture was stirred at room temperature $\left(25-30^{\circ} \mathrm{C}\right)$ for $6 \mathrm{~d}$. After releasing the hydrogen pressure, the reaction mixtures were concentrated in vacuo, and purified by flash chromatography on silica gel with petroleum ether/ethyl acetate (5:1) as an eluent to provide $(R)$ $3 \mathbf{i}$ as a colorless oil in $96 \%$ yield ( $3.26 \mathrm{~g}, 98 \%$ conversion) with $91 \%$ ee.

\section{Synthesis of $(R)$-3-(4-phenylphenyl)butanoic acid $((R)-8)^{17}$}

According to the aforementioned procedure for the synthesis of $(R)-\mathbf{5},(R)-\mathbf{3 i}(1.70 \mathrm{~g}, 5 \mathrm{mmol})$ was converted to $(R)-8$ as a white solid. $1.10 \mathrm{~g}, 92 \%$ yield, $91 \%$ ee ( $84 \%$ yield, $95 \%$ ee, after recrystallized in a mixed solvent of petroleum ether and ethyl acetate (5:1)). $[\alpha]_{\mathrm{D}}^{26}-36.5\left(c 1.0, \mathrm{CH}_{3} \mathrm{OH}\right) ;$ lit. $^{17}[\alpha]_{\mathrm{D}}^{20}$ $+33.6\left(c 1.0, \mathrm{CH}_{3} \mathrm{OH}\right), 87 \%$ ee for $(S)$-isomer). ${ }^{1} \mathrm{H}$ NMR $\left(400 \mathrm{MHz}, \mathrm{CDCl}_{3}\right) \delta: 11.66(\mathrm{~s}, 1 \mathrm{H}), 7.55(\mathrm{dd}, J$ $=15.7,7.9 \mathrm{~Hz}, 4 \mathrm{H}), 7.42(\mathrm{t}, J=7.5 \mathrm{~Hz}, 2 \mathrm{H}), 7.31(\mathrm{dd}, J=15.7,7.7 \mathrm{~Hz}, 3 \mathrm{H}), 3.38-3.26(\mathrm{~m}, 1 \mathrm{H}), 2.71$ (dd, $J=15.6,6.8 \mathrm{~Hz}, 1 \mathrm{H}), 2.61(\mathrm{dd}, J=15.6,8.2 \mathrm{~Hz}, 1 \mathrm{H}), 1.35(\mathrm{~d}, J=6.9 \mathrm{~Hz}, 3 \mathrm{H})$.

\section{(E) Computational Study}

\section{DFT Methods.}

All DFT calculations have been carried out using the Gaussian 16 program package. ${ }^{23}$ The B3LYP ${ }^{24}$ method with def2svp basis set has been selected for geometry optimizations and calculation of Gibbs energy corrections at $298 \mathrm{~K}$. Final energies were retrieved from single-point calculations at the B3LYP/6$311+\mathrm{G}^{* *}$ level, including the GD3BJ dispersion correction scheme developed by Grimme. ${ }^{25}$ All structures have been optimized considering solvent effects using the CPCM Model for MeOH. Reaction paths were traced by the intrinsic reaction coordinate method for all transition states. All energetics reported throughout the text are in $\mathrm{kcal} / \mathrm{mol}$. Structures were generated using CYLview. ${ }^{26}$

\section{DFT Structures}

The absolute configuration of iridium catalyst is determined by crystal structure. The chiral iridiumhydride intermediates can be generated in situ from the reaction mixture, and may act as an effective reductant for the upper-face or down-face attack on the highly activated $\mathrm{C}=\mathrm{C}$ bond of the substrate via a six-membered cyclic transition (TS), assuming a Noyori bifunctional mechanism like ketones.

(a)

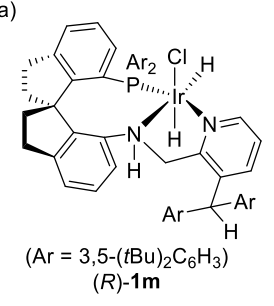

(b)

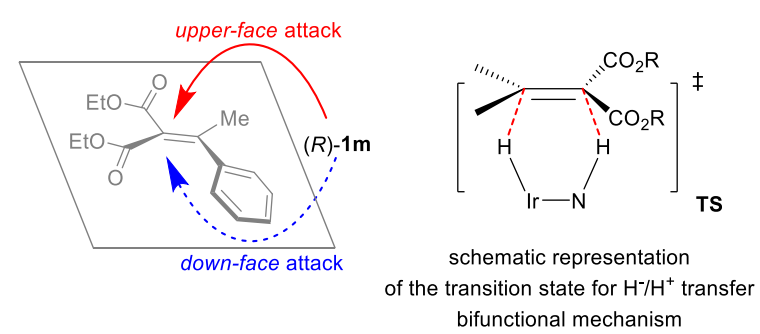

The two models of transition state structures for the $\mathrm{H}^{-} / \mathrm{H}^{+}$transfer to 2 a were simulated in Figure $\mathrm{S} 1$. In these two processes, the bulky group at the 3-position of the pyridine ring and the rigid spiro backbone of the catalyst direct the approach of the substrate to the catalyst. Both of the TS-R (down-face attack) and TS-S (upper-face attack) suffering from the steric hindrance between the ester group and substituents at the 3-position of the pyridine ring; while in TS-S, another steric hindrance between phenyl ring of the substrate and the substituents of phosphine atom and the rigid spiro backbone further increases the difficulty of the interaction between the substrate and the catalyst. 


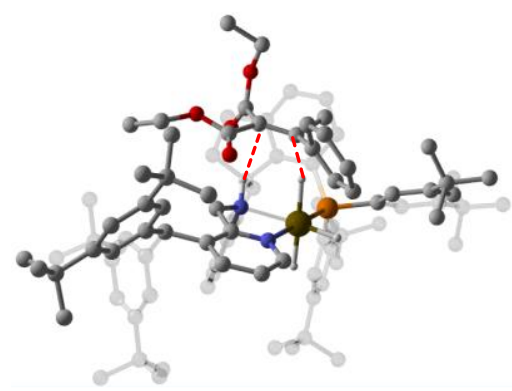

TS- $R\left(0.00 \mathrm{kcal} \mathrm{mol}^{-1}\right)$

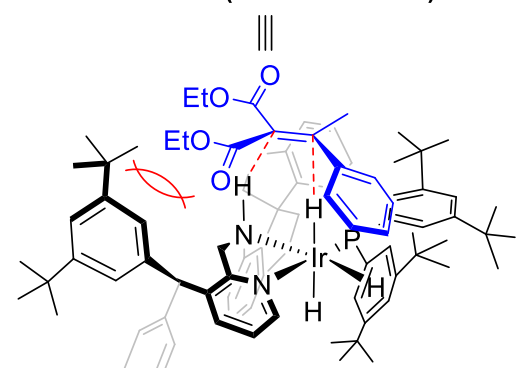

favorable

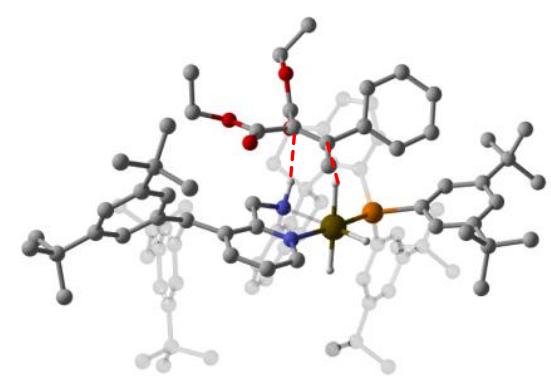

TS-S (2.91 kcal mol-1)

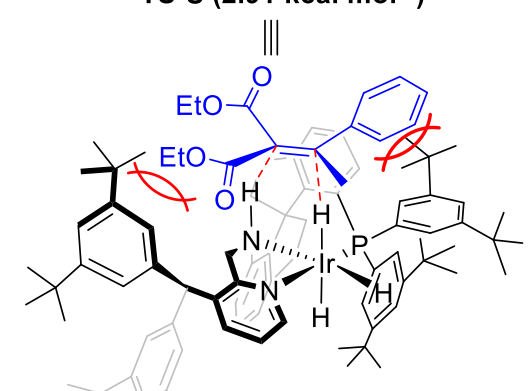

unfavorable

Figure S1. The transition state structures for the $\mathrm{H}^{-} / \mathrm{H}^{+}$transfer to $\mathbf{2 a}$

For the model of the interaction between the substrate $\mathbf{2 a}$ and catalyst, the Boltzmann analysis of the two competing TS structures were summarized (Table S2). The calculation of each TS structure based on the Boltzmann distribution, indicated that TS- $R$ is preferable than TS- $S$, which was found lying $2.91 \mathrm{kcal} / \mathrm{mol}$ in free activation energy, leading to formation of $(R)-\mathbf{3 a}$ as the major product with a calculated $98 \% e e$, which is in good agreement with the experimental results $(94 \%$ ee $)$.

Table S2. DFT-calculated Boltzmann distribution of the TS structures for $(R)$-Ir-H catalyzed hydride/proton transfer to $\mathbf{2 a}$

\begin{tabular}{lll}
\hline Transition States & Free energy in solution (a.u.) & relative $\mathrm{G}(\mathrm{kcal} / \mathrm{mol})$ \\
\hline TS- $S$ & -4548.02628 & 2.91476 \\
TS- $R$ & -4548.03092 & 0.00000 \\
\hline & $\operatorname{Ee}(\%)$ & 0.98554 \\
\hline
\end{tabular}




\section{References}

(1) Xie, J.-H.; Liu, X.-Y.; Xie, J.-B.; Wang, L.-X.; Zhou, Q.-L. An Additional Coordination Group Leads to Extremely Efficient Chiral Iridium Catalysts for Asymmetric Hydrogenation of Ketones. Angew. Chem., Int. Ed. 2011, 50, 7329.

(2) Ashida, Y.; Sato, Y.; Suzuki, T.; Ueno, K.; Kai, K.-i.; Nakatsuji, H.; Tanabe, Y. (E)-,(Z)-Parallel Preparative Methods for Stereodefined $\beta, \beta$-Diaryl- and $\alpha, \beta$-Diaryl- $\alpha, \beta$-unsaturated Esters: Application to the Stereocomplementary Concise Synthesis of Zimelidine. Chem. Eur. J. 2015, 21, 5934.

(3) Zhang, J.; Blazecka, P. G.; Angell, P.; Lovdahl, M.; Curran, T. T. Indium(III)-Mediated Markovnikov Addition of Malonates and $\beta$-Ketoesters to Terminal Alkynes and the Formation of Knoevenagel Condensation Products. Tetrahedron 2005, 61, 7807.

(4) Jung, J. C.; Watkins, E. B.; Avery, M. A. Efficient Synthesis of 4-Ethoxycarbonyl Pyrazolin-5-one Derivatives. Synth. Commun. 2002, 32, 3767.

(5) Rathke, M. W.; Cowan, P. J. Procedures for the Acylation of Diethyl Malonate and Ethyl Acetoacetate with Acid Chlorides Using Tertiary Amine Bases and Magnesium Chloride. J. Org. Chem. $\mathbf{1 9 8 5}, 50,2622$.

(6) Uozumi, T.; Yamada, S.; Nakamura, N.; Hisano, K.; Hosaka, M.; Sugano, T. Manufacture of Solid Catalyst Components for Olefin Polymerization, Catalysts for Olefin Polymerization, and Olefin Polymers Therewith. WO2013005463A1, 2013.

(7) Miersch, A.; Harms, K.; Hilt, G., Zinc-Mediated Addition of Diethyl Bromomalonate to Alkynes for the Cascade Reaction Towards Polysubstituted Pyranones and Tetracarbonyl Derivatives. Chem. Commun. 2014, 50, 542.

(8) Poondra, R. R.; Fischer, P. M.; Turner, N. J., Efficient Palladium-Catalyzed Cross-Coupling of $\beta$ Chloroalkylidene/arylidene Malonates Using Microwave Chemistry. J. Org. Chem. 2004, 69, 6920.

(9) Cahiez, G.; Alami, M., Organomanganese (II) Reagents XV. Conjugate Addition of Organomanganese Reagents to Alkylidenemalonic Esters and Related Compounds. Tetrahedron 1989, $45,4163$.

(10) Metallinos, C.; Van Belle, L. Asymmetric Hydrogenation of Alkenes with Planar Chiral 2Phosphino-1-aminoferrocene-iridium(I) Complexes. J. Organometal. Chem. 2011, 696, 141.

(11) Alexakis, A.; Benhaim, C. Asymmetric Conjugate Addition to Alkylidene Malonates. Tetrahedron: Asymmetry 2001, 12, 1151.

(12) Patra, A.; Misra, S. K. A New Synthesis of 4-Substituted Coumarins. Indian J. Chem., Sect. B 1990, $29 B, 66$.

(13) Deasy, R. E.; Brossat, M.; Moody, T. S.; Maguire, A. R. Lipase Catalysed Kinetic Resolutions of 3Aryl Alkanoic Acids. Tetrahedron: Asymmetry 2011, 22, 47.

(14) Kelkar, S. V.; Joshi, G. S.; Kulkarni, G. H.; Mitra, R. B. Synthesis of 3-Phenoxybenzyl 3-Alkyl-3( $p$-substituted phenyl)propionates and 3-Phenoxybenzyl 2-Alkyl-3-( $p$-substituted phenyl)propionates. Indian J. Chem., Sect. B 1987, 26B, 68.

(15) Guo, S.; Zhou, J. N,N-Dimethylformamide as Hydride Source in Nickel-Catalyzed Asymmetric Hydrogenation of $\alpha, \beta$-Unsaturated Esters. Org. Lett. 2016, 18, 5344.

(16) Liu, W.; Liu, P.; Lv, L.; Li, C.-J. Metal-Free and Redox-Neutral Conversion of Organotrifluoroborates into Radicals Enabled by Visible Light. Angew. Chem. Int. Ed. 2018, 57, 13499.

(17) Rossi, D.; Pedrali, A.; Gaggeri, R.; Marra, A.; Pignataro, L.; Laurini, E.; DalCol, V.; Fermeglia, M.; Pricl, S.; Schepmann, D.; Wünsch, B.; Peviani, M.; Curti, D.; Collina, S. Chemical, Pharmacological, and in vitro Metabolic Stability Studies on Enantiomerically Pure RC-33 Compounds: Promising 
Neuroprotective Agents Acting as $\sigma_{1}$ Receptor Agonists. ChemMedChem 2013, 8, 1514.

(18) Itooka, R.; Iguchi, Y.; Miyaura, N. Rhodium-Catalyzed 1,4-Addition of Arylboronic Acids to $\alpha, \beta$ Unsaturated Carbonyl Compounds: Large Accelerating Effects of Bases and Ligands. J. Org. Chem. 2003, 68, 6000 .

(19) Schuppan, J.; Minnaard, A. J.; Feringa, B. L. A Catalytic and Iterative Route to $\beta$-Substituted Esters via Highly Enantioselective Conjugate Addition of Dimethylzinc to Unsaturated Malonates. Chem. Commun. 2004, 792.

(20) Xue, F.; Wang, F.; Liu, J.; Di, J.; Liao, Q.; Lu, H.; Zhu, M.; He, L.; He, H.; Zhang, D.; Song, H.; Liu, X.-Y.; Qin, Y. A Desulfurative Strategy for the Generation of Alkyl Radicals Enabled by VisibleLight Photoredox Catalysis. Angew. Chem., Int. Ed. 2018, 57, 6667.

(21) Nicolas, E.; Russell, K. C.; Hruby, V. J. Asymmetric 1,4-Addition of Organocuprates to chiral $\alpha, \beta$ Unsaturated N-Acyl-4-phenyl-2-oxazolidinones: A New Approach to the Synthesis of Chiral $\beta$-Branched Carboxylic Acids. J. Org. Chem. 1993, 58, 766.

(22) Fournie-Zaluski, M.-C.; Coric, P.; Turcaud, S.; Rousselet, N.; Gonzalez, W.; Barbe, B.; Pham, I.; Jullian, N.; Michel, J.-B. Roques, B. P., New Dual Inhibitors of Neutral Endopeptidase and AngiotensinConverting Enzyme: Rational Design, Bioavailability, and Pharmacological Responses in Experimental Hypertension. J. Med. Chem. 1994, 37, 1070.

(23) Frisch, M. J.; Trucks, G. W.; Schlegel, H. B.; Scuseria, G. E.; Robb, M. A.; Cheeseman, J. R.; Scalmani, G.; Barone, V.; Petersson, G. A.; Nakatsuji, H.; Li, X.; Caricato, M.; Marenich, A. V.; Bloino, J.; Janesko, B. G.; Gomperts, R.; Mennucci, B.; Hratchian, H. P.; Ortiz, J. V.; Izmaylov, A. F.; Sonnenberg, J. L.; Williams-Young, F.; Ding, F.; Lipparini, F.; Egidi, F.; Goings, J.; Peng, B.; Petrone, A.; Henderson, T.; Ranasinghe, D.; Zakrzewski, V. G.; Gao, J.; Rega, N.; Zheng, G.; Liang, W.; Hada, M.; Ehara, M.; Toyota, K.; Fukuda, R.; Hasegawa, J.; Ishida, M.; Nakajima, T.; Honda, Y.; Kitao, O.; Nakai, H.; Vreven, T.; Throssell, K.; Montgomery Jr., J. A.; Peralta, J. E.; Ogliaro, F.; Bearpark, M. J.; Heyd, J. J.; Brothers, E. N.; Kudin, K. N.; Staroverov, V. N.; Keith, T. A.; Kobayashi, R.; Normand, J.; Raghavachari, K.; Rendell, A. P.; Burant, J. C.; Iyengar, S. S.; Tomasi, J.; Cossi, M.; Millam, J. M.; Klene, M.; Adamo, C.; Cammi, R.; Ochterski, J. W.; Martin, R. L.; Morokuma, K.; Farkas, O.; Foresman, J. B.; Fox, D. J. Gaussian 16, Revision A.03; Wallingford, CT, 2016.

(24) (a) Lee, C.; Yang, W.; Parr, R. G. Development of the Colle-Salvetti Correlation-Energy Formula into a Functional of the Electron density. Phys. Rev. B. 1988, 37, 785. (b) Becke, A. D. A New Mixing of Hartree-Fock and Local Density-functional theories. J. Chem. Phys. 1993, 98, 1372. (c) Becke, A. D. Density Functional Thermochemistry. III. The Role of Exact Exchange. J. Chem. Phys. 1993, 98, 5648. (25) Grimme, S.; Antony, J.; Ehrlich, S.; Krieg, H. A Consistent and Accurate ab Initio Parametrization of Density Functional Dispersion Correction (DFT-D) for the 94 Elements H-Pu. J. Chem. Phys. 2010, $132,154104$.

(26) Legault, C. Y., CYLview, 1.0b (Université de Sherbrooke, 2009). 
(F) NMR Spectra of New Compounds

(2-Methylpyridin-3-yl)diphenylmethanol (S1a)

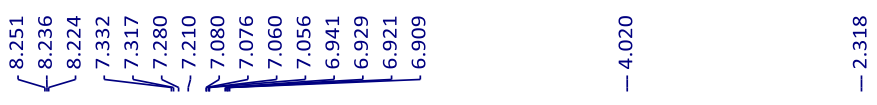<smiles>OC(c1ccccc1)(c1ccccc1)c1cccnc1</smiles>

S1a

$\left({ }^{1} \mathrm{H} \mathrm{NMR}, 400 \mathrm{MHz}, \mathrm{CDCl}_{3}\right)$

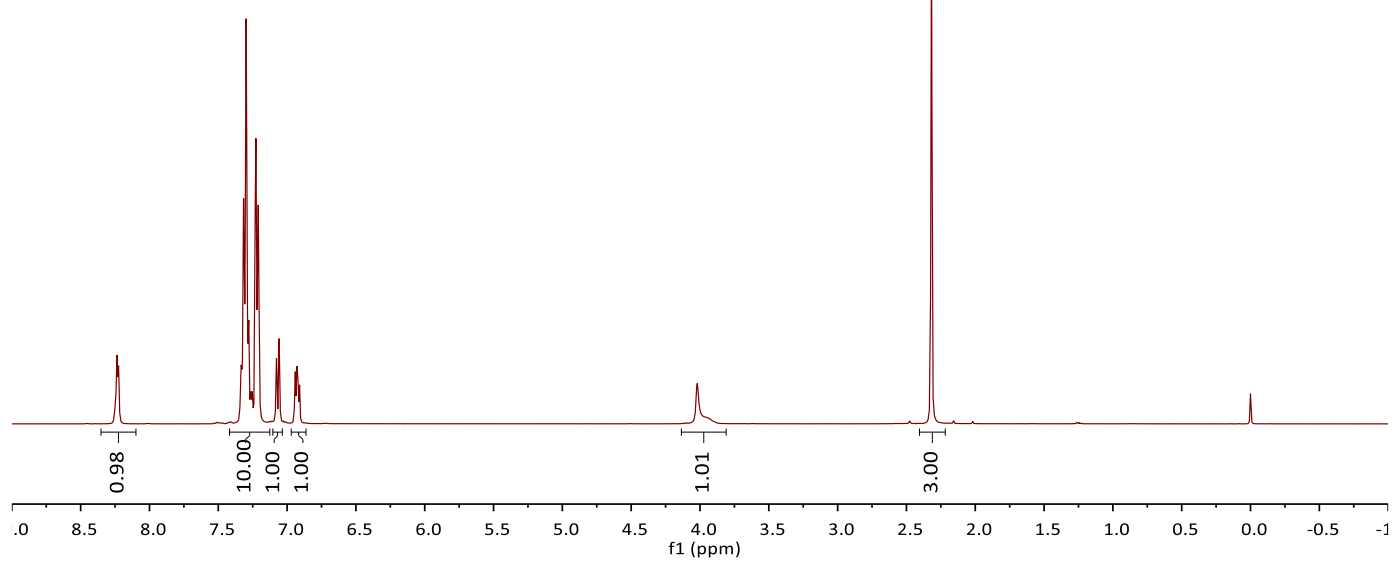

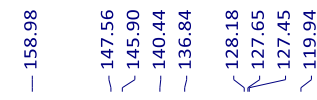
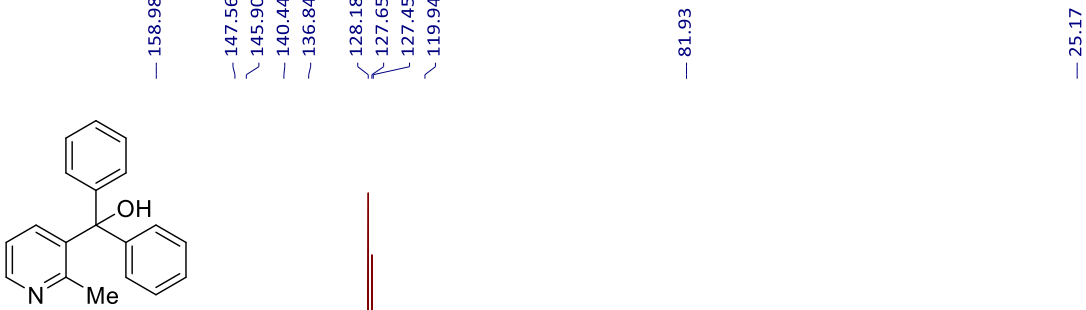

S1a

$\left({ }^{13} \mathrm{C} \mathrm{NMR}, 101 \mathrm{MHz}, \mathrm{CDCl}_{3}\right)$

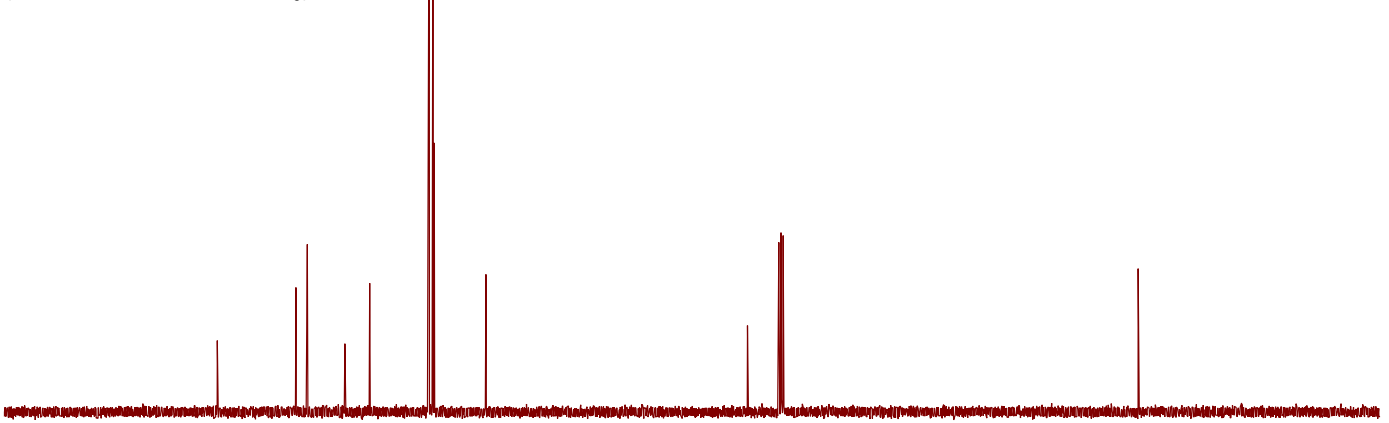

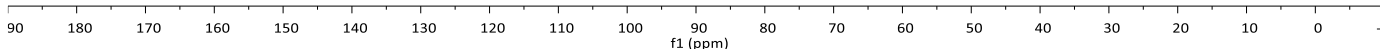


Bis(3,5-dimethylphenyl)(2-methylpyridin-3-yl)methanol (S1b)

$\underbrace{\substack{0.0 \\ 0.0}}$

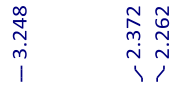

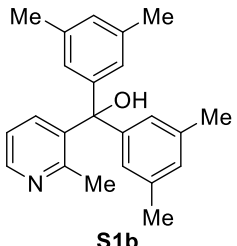

$\left({ }^{1} \mathrm{H} \mathrm{NMR}, 400 \mathrm{MHz}, \mathrm{CDCl}_{3}\right.$ )

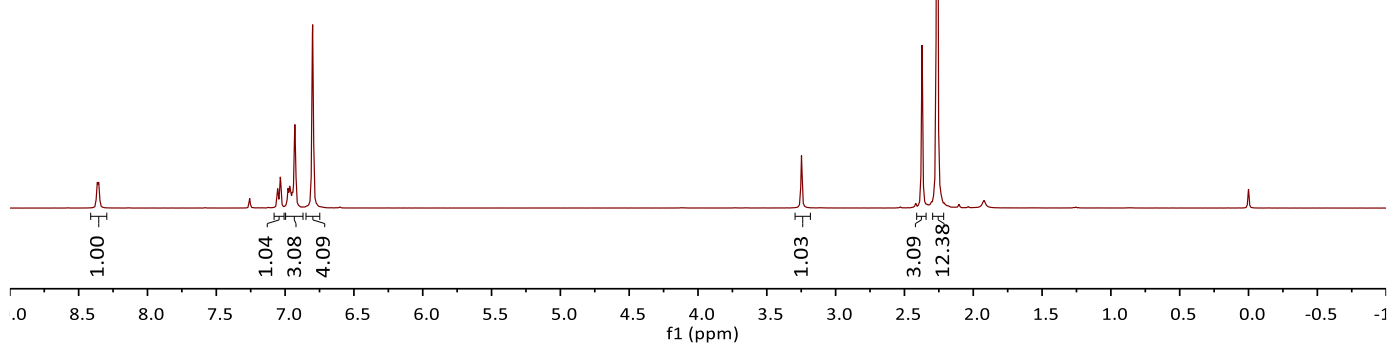

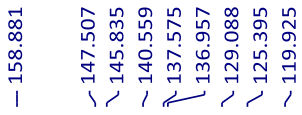
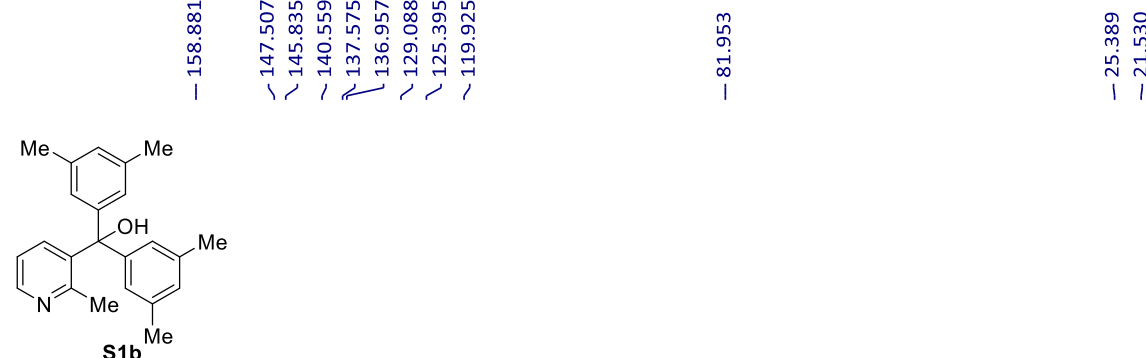

$\left({ }^{13} \mathrm{C}\right.$ NMR, $\left.101 \mathrm{MHz}, \mathrm{CDCl}_{3}\right)$

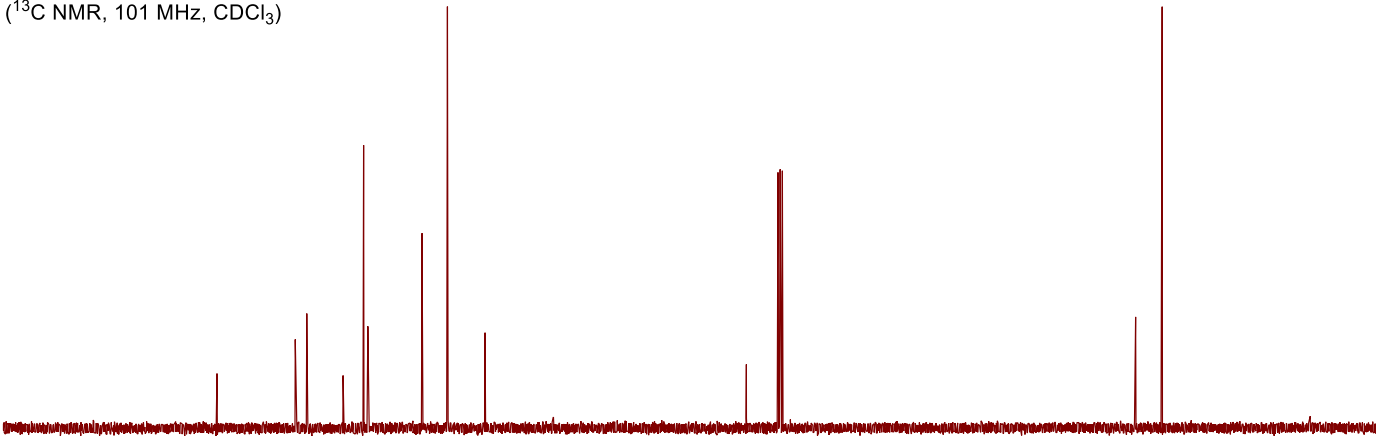

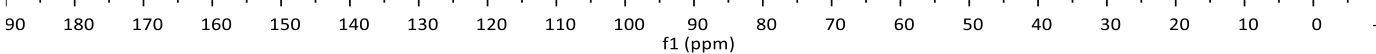


Bis(3,5-diisopropylphenyl)(2-methylpyridin-3-yl)methanol (S1c)
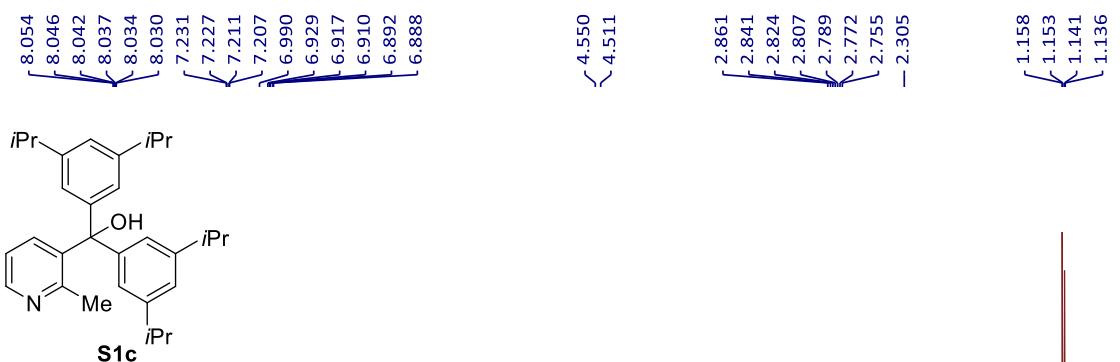

$\left({ }^{1} \mathrm{H} \mathrm{NMR}, 400 \mathrm{MHz}, \mathrm{CDCl}_{3}\right)$

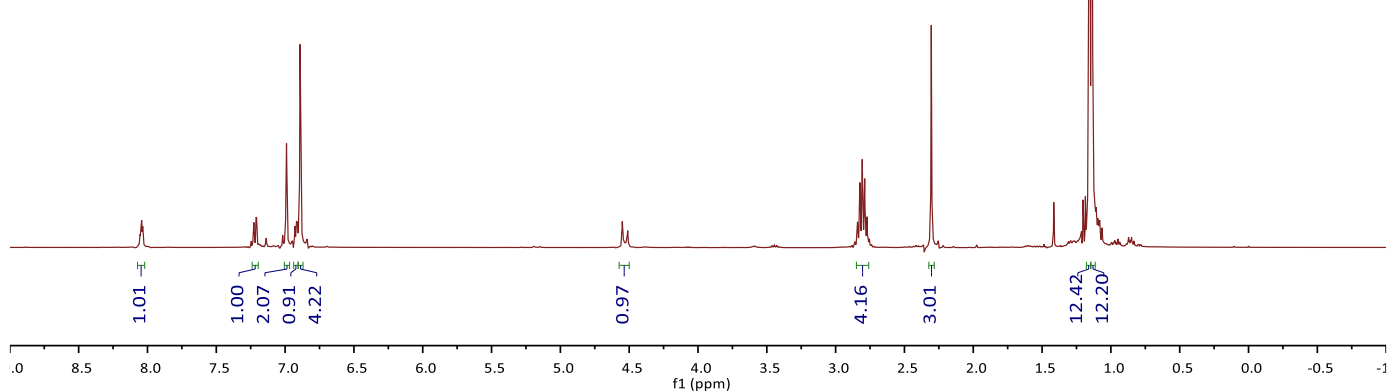

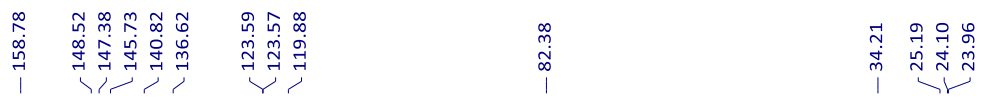

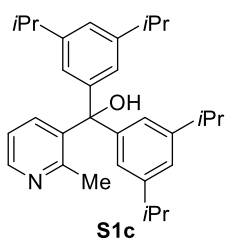

$\left({ }^{13} \mathrm{C}\right.$ NMR, $\left.101 \mathrm{MHz}, \mathrm{CDCl}_{3}\right)$

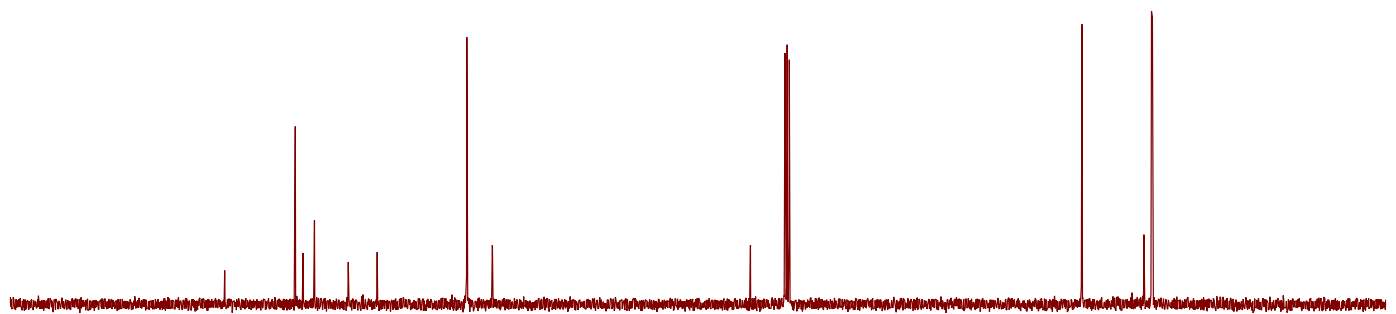

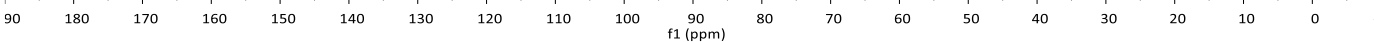


Bis(3,5-di-tert-butylphenyl)(2-methylpyridin-3-yl)methanol (S1d)

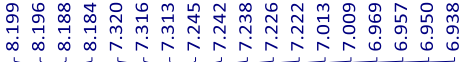

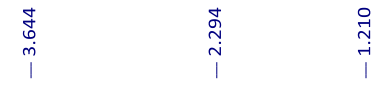

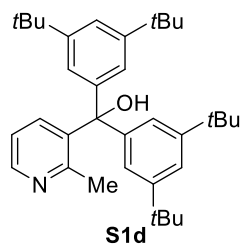

$\left({ }^{1} \mathrm{H} \mathrm{NMR}, 400 \mathrm{MHz}, \mathrm{CDCl}_{3}\right)$
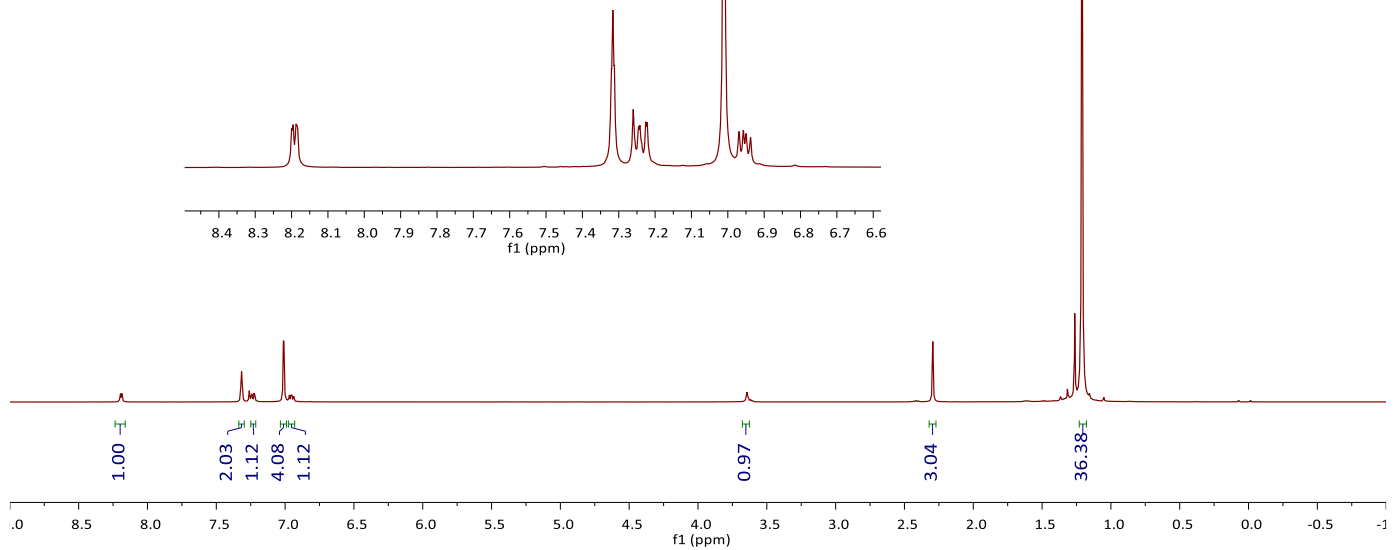

\begin{tabular}{|c|c|}
\hline 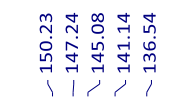 & 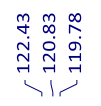 \\
\hline
\end{tabular}

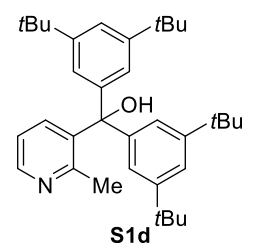

$\left({ }^{13} \mathrm{C} \mathrm{NMR}, 101 \mathrm{MHz}, \mathrm{CDCl}_{3}\right)$

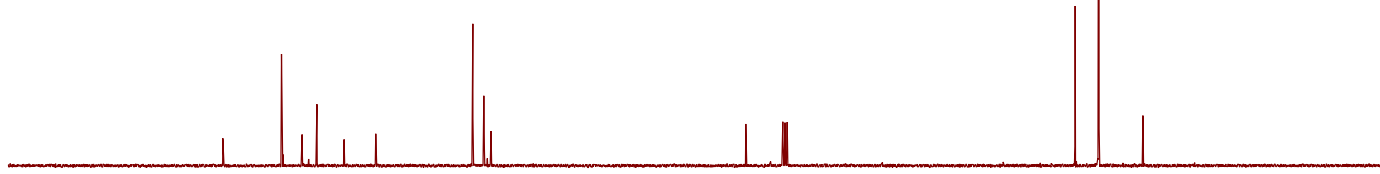

\begin{tabular}{lllllllllllllllllllllll}
\hline 90 & 180 & 170 & 160 & 150 & 140 & 130 & 120 & 110 & 100 & 90 & 80 & 70 & 60 & 50 & 40 & 30 & 20 & 10 & 0 & 10
\end{tabular} 
3-Benzhydryl-2-methylpyridine (S2a)

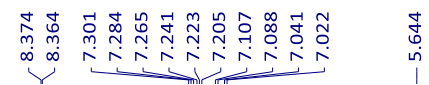

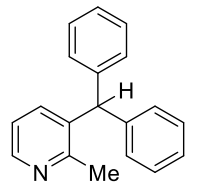

S2a

$\left({ }^{1} \mathrm{H} \mathrm{NMR}, 400 \mathrm{MHz}, \mathrm{CDCl}_{3}\right)$

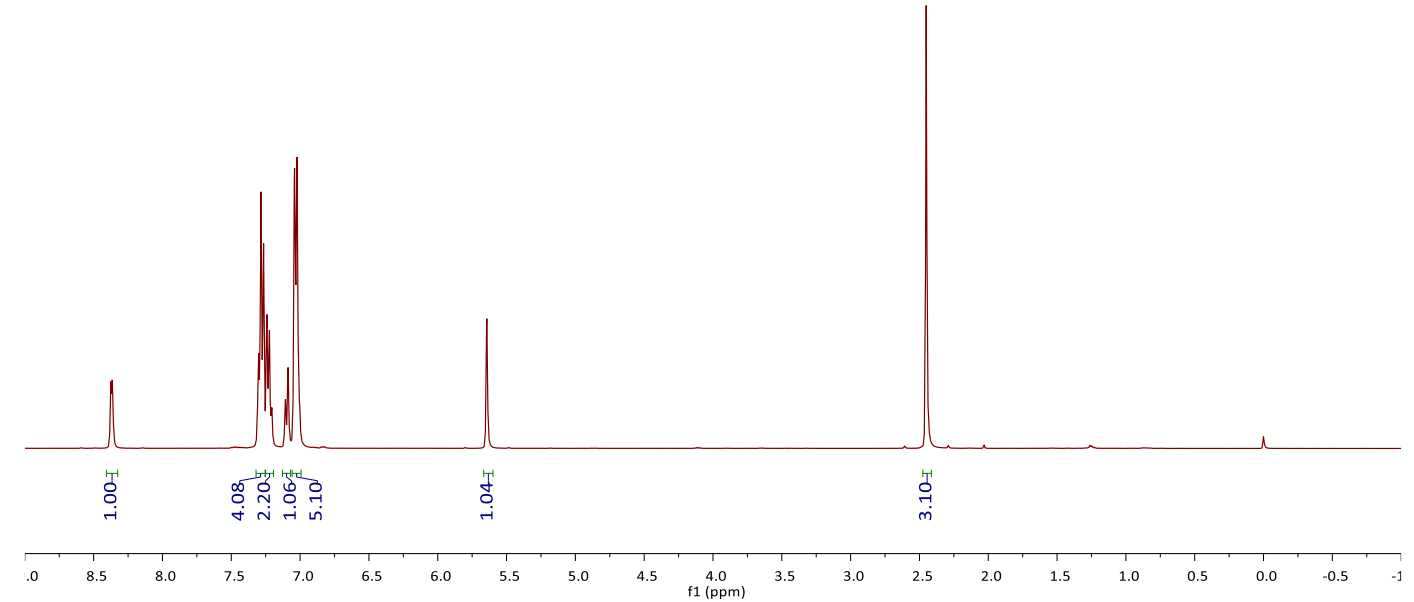

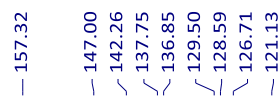

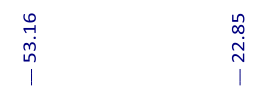

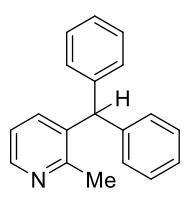

S2a

$\left({ }^{13} \mathrm{C} \mathrm{NMR}, 101 \mathrm{MHz}, \mathrm{CDCl}_{3}\right)$

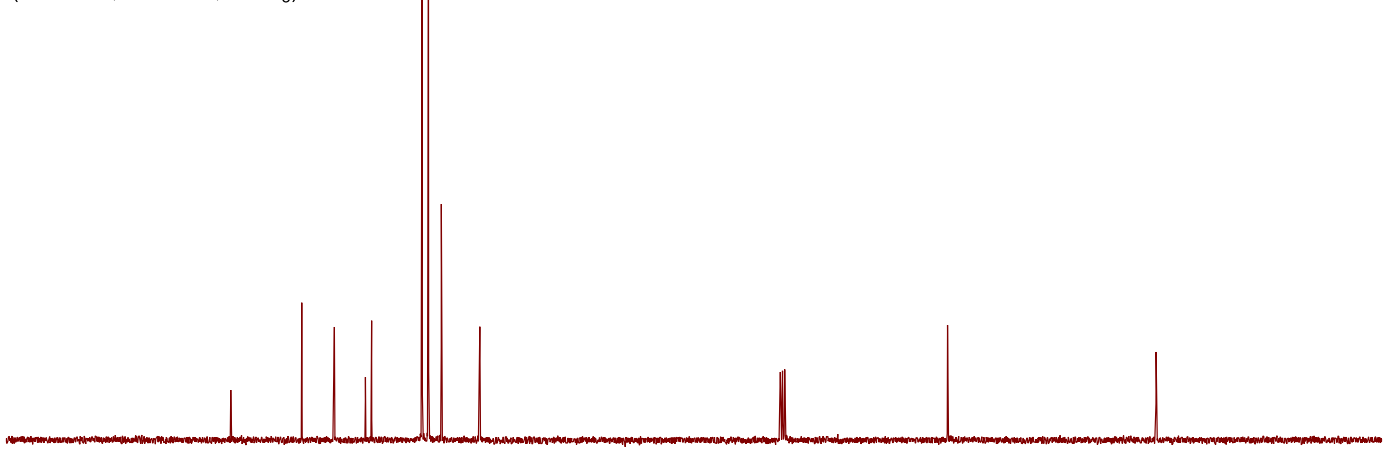

\begin{tabular}{lllllllllllllllllllllllll}
\hline 90 & 180 & 170 & 160 & 150 & 140 & 130 & 120 & 110 & 100 & 90 & 80 & 70 & 60 & 50 & 40 & 30 & 20 & 10 & 0 & $-:$
\end{tabular} 
3-(Bis(3,5-dimethylphenyl)methyl)-2-methylpyridine (S2b)

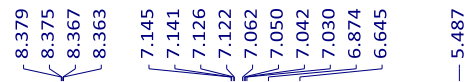

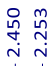

$\mathrm{Me}^{\mathrm{Me}}$

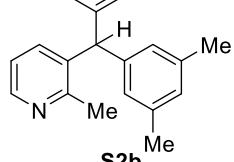

$\mathrm{S}^{\mathrm{b}}{ }^{\mathrm{Me}}$

( ${ }^{1} \mathrm{H}$ NMR, $400 \mathrm{MHz}, \mathrm{CDCl}_{3}$ )

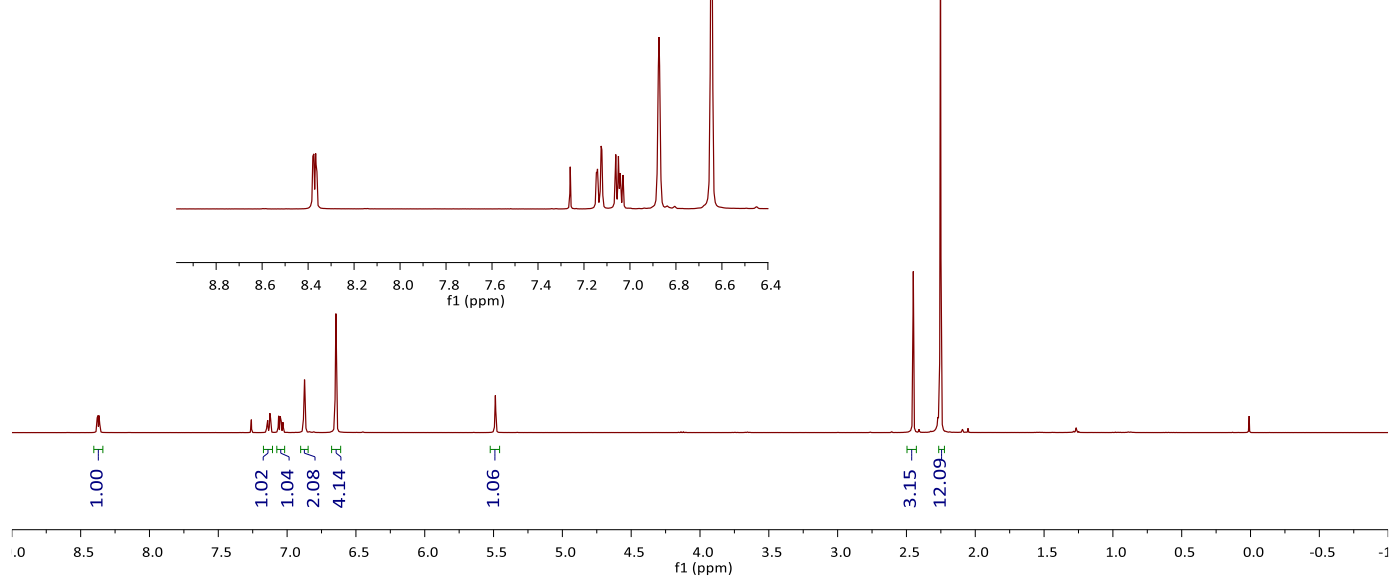

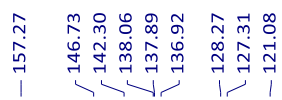
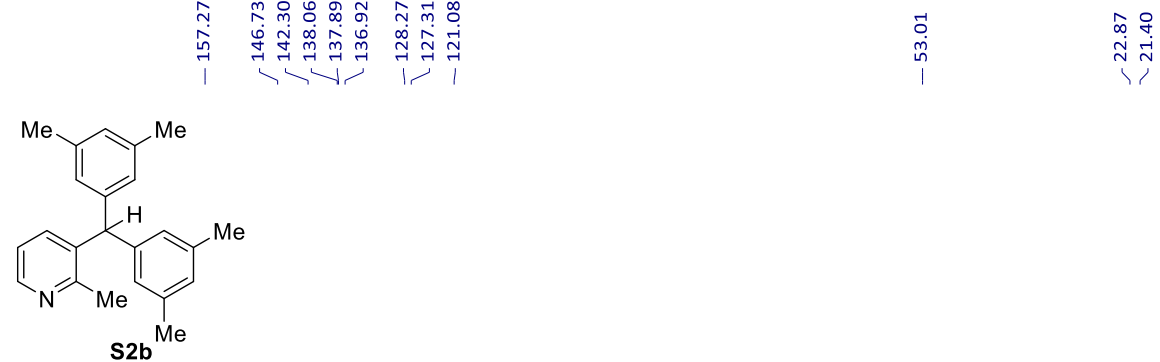

$\left({ }^{13} \mathrm{C}\right.$ NMR, $\left.101 \mathrm{MHz}, \mathrm{CDCl}_{3}\right)$

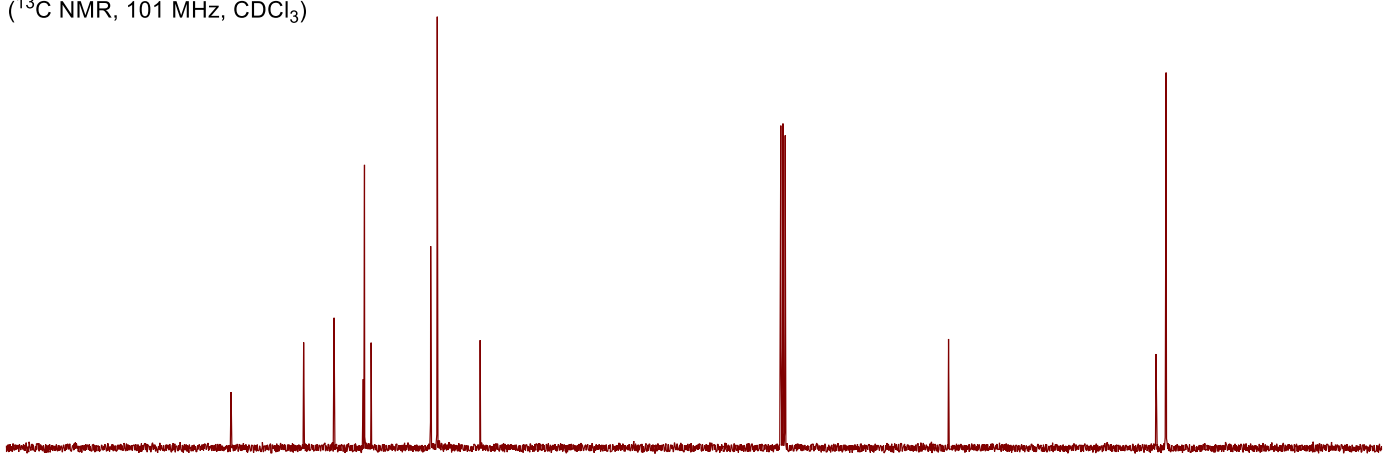

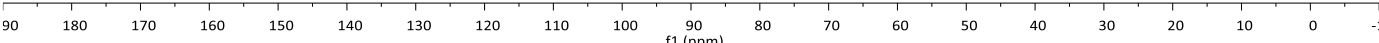


3-(Bis(3,5-diisopropylphenyl)methyl)-2-methylpyridine (S2c)
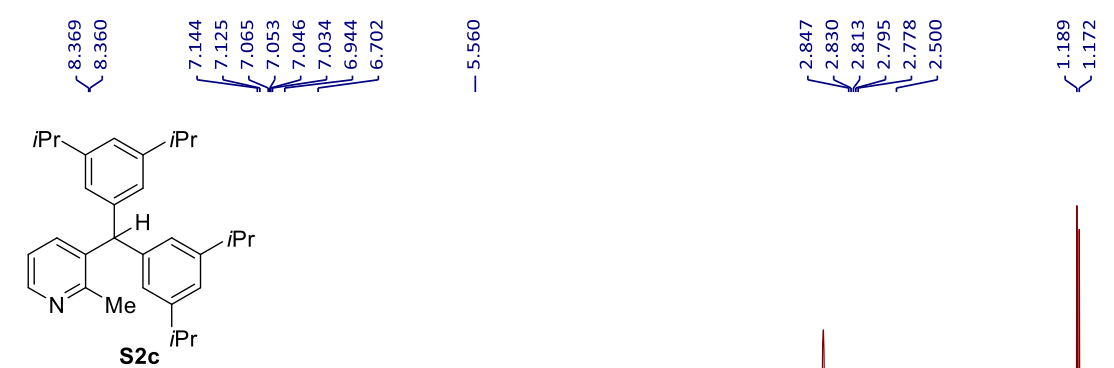

( ${ }^{1} \mathrm{H}$ NMR, $400 \mathrm{MHz}, \mathrm{CDCl}_{3}$ )
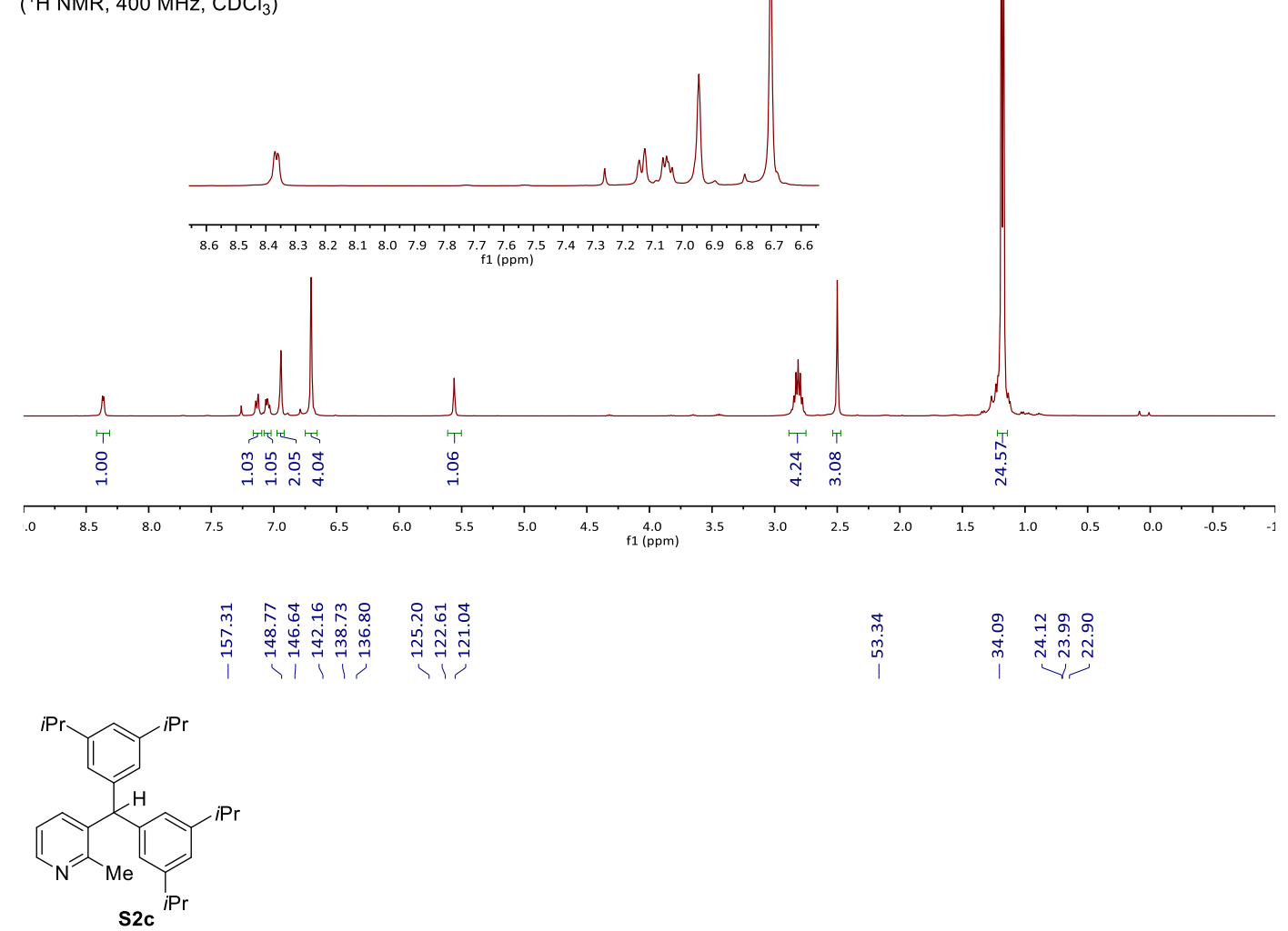

$\left({ }^{13} \mathrm{C}\right.$ NMR, $\left.101 \mathrm{MHz}, \mathrm{CDCl}_{3}\right)$
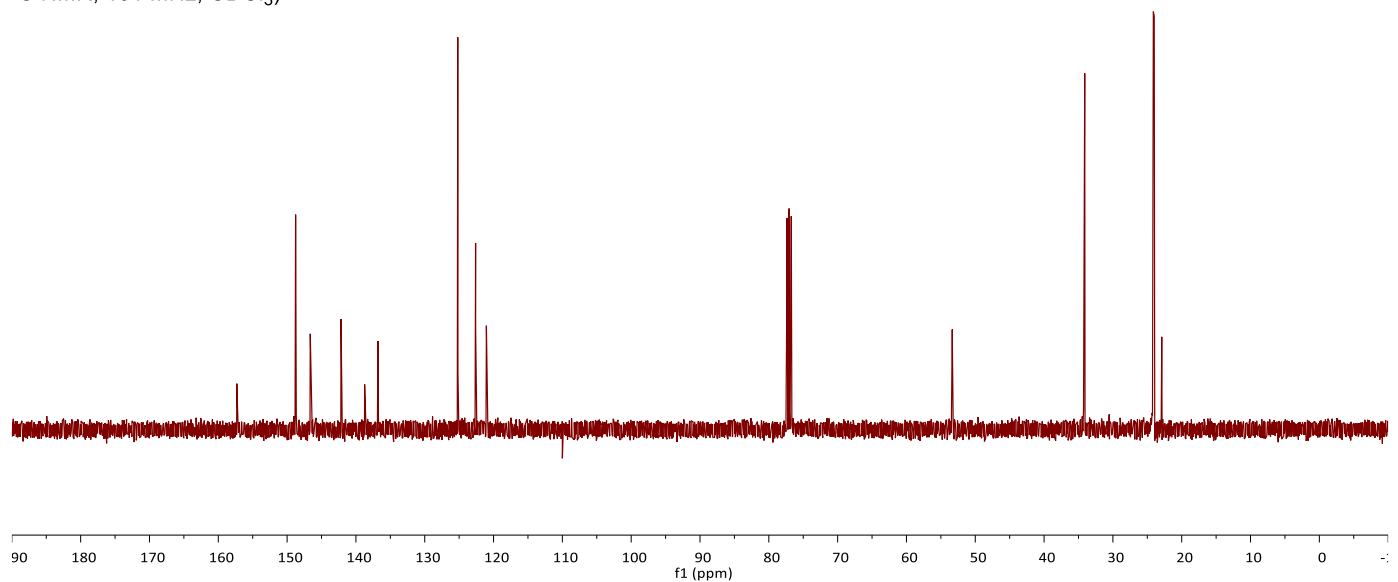
3-(Bis(3,5-di-tert-butylphenyl)methyl)-2-methylpyridine (S2d)

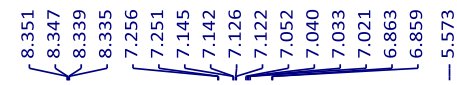

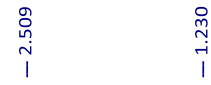

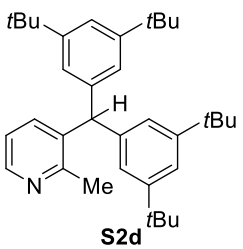

$\left({ }^{1} \mathrm{H} \mathrm{NMR}, 400 \mathrm{MHz}, \mathrm{CDCl}_{3}\right)$

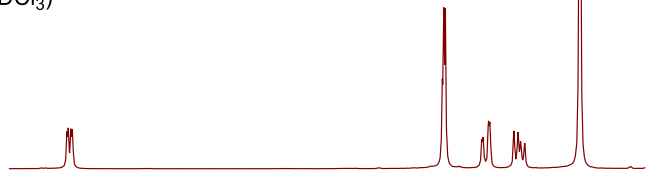

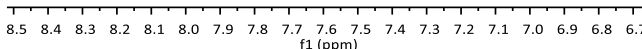

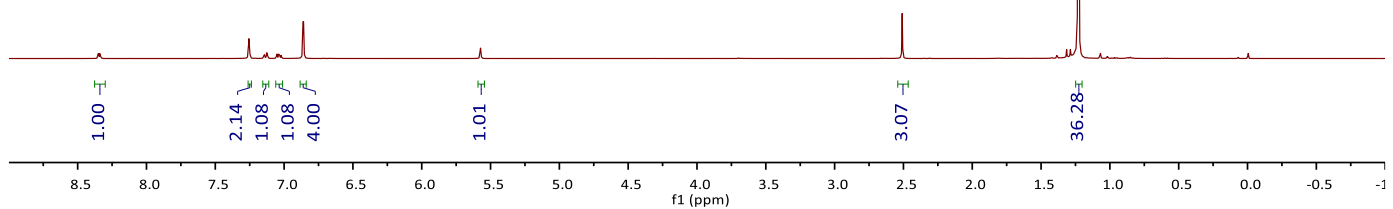

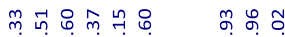

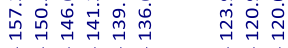

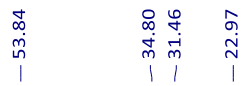

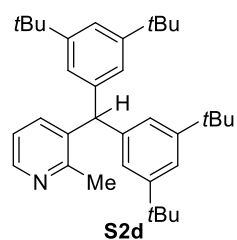

$\left({ }^{13} \mathrm{C} \mathrm{NMR}, 101 \mathrm{MHz}, \mathrm{CDCl}_{3}\right)$

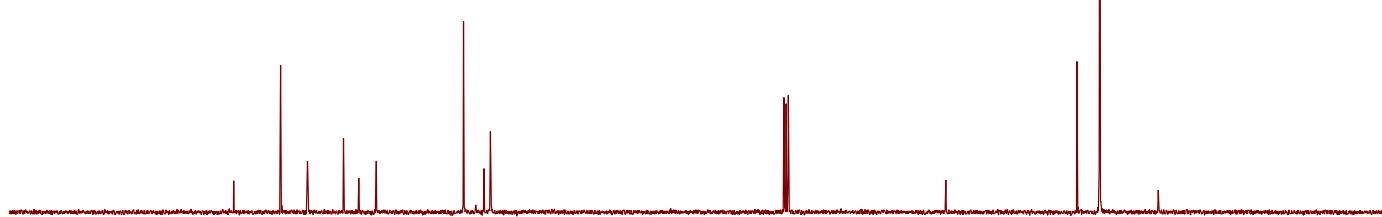

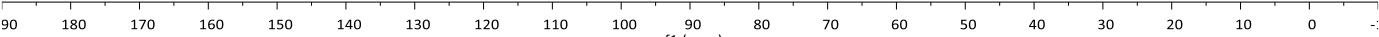


3-Benzhydrylpicolinaldehyde (S3a)

策

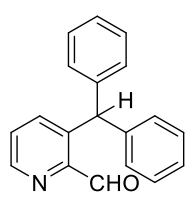

S3a

$\left({ }^{1} \mathrm{H} \mathrm{NMR}, 400 \mathrm{MHz}, \mathrm{CDCl}_{3}\right)$

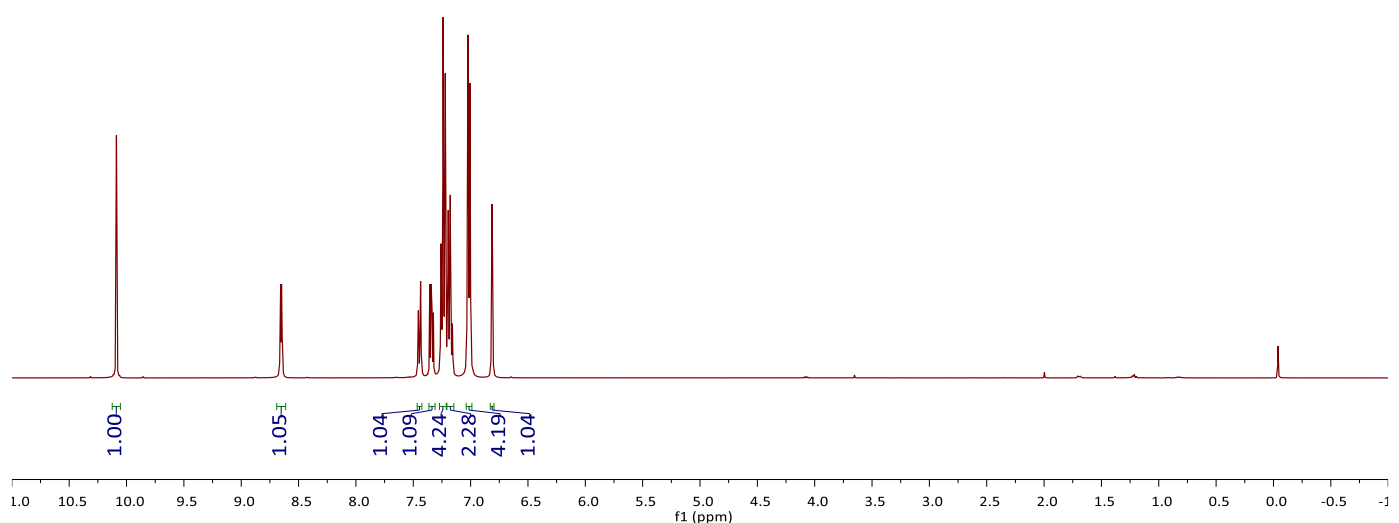

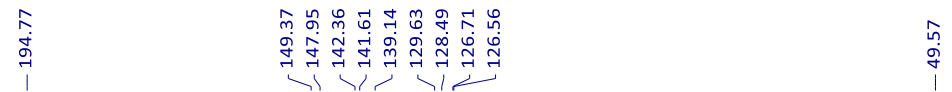

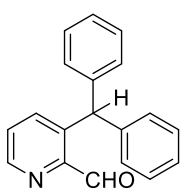

s3a

$\left({ }^{13} \mathrm{C} \mathrm{NMR}, 101 \mathrm{MHz}, \mathrm{CDCl}_{3}\right)$
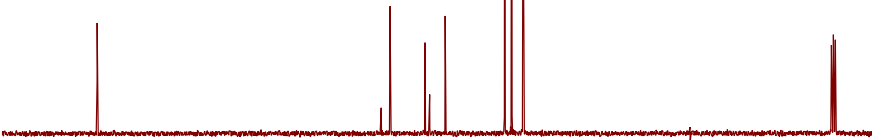

$10 \quad 200$

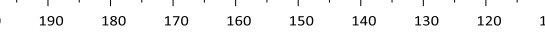

$10 \underset{100}{100} 90$ 
3-(Bis(3,5-dimethylphenyl)methyl)picolinaldehyde (S3b)

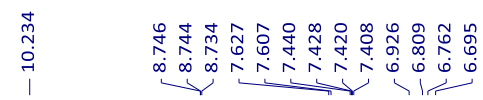

$\stackrel{\substack{p \\ i}}{i}$

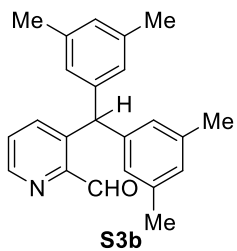

( ${ }^{1} \mathrm{H}$ NMR, $400 \mathrm{MHz}, \mathrm{CDCl}_{3}$ )
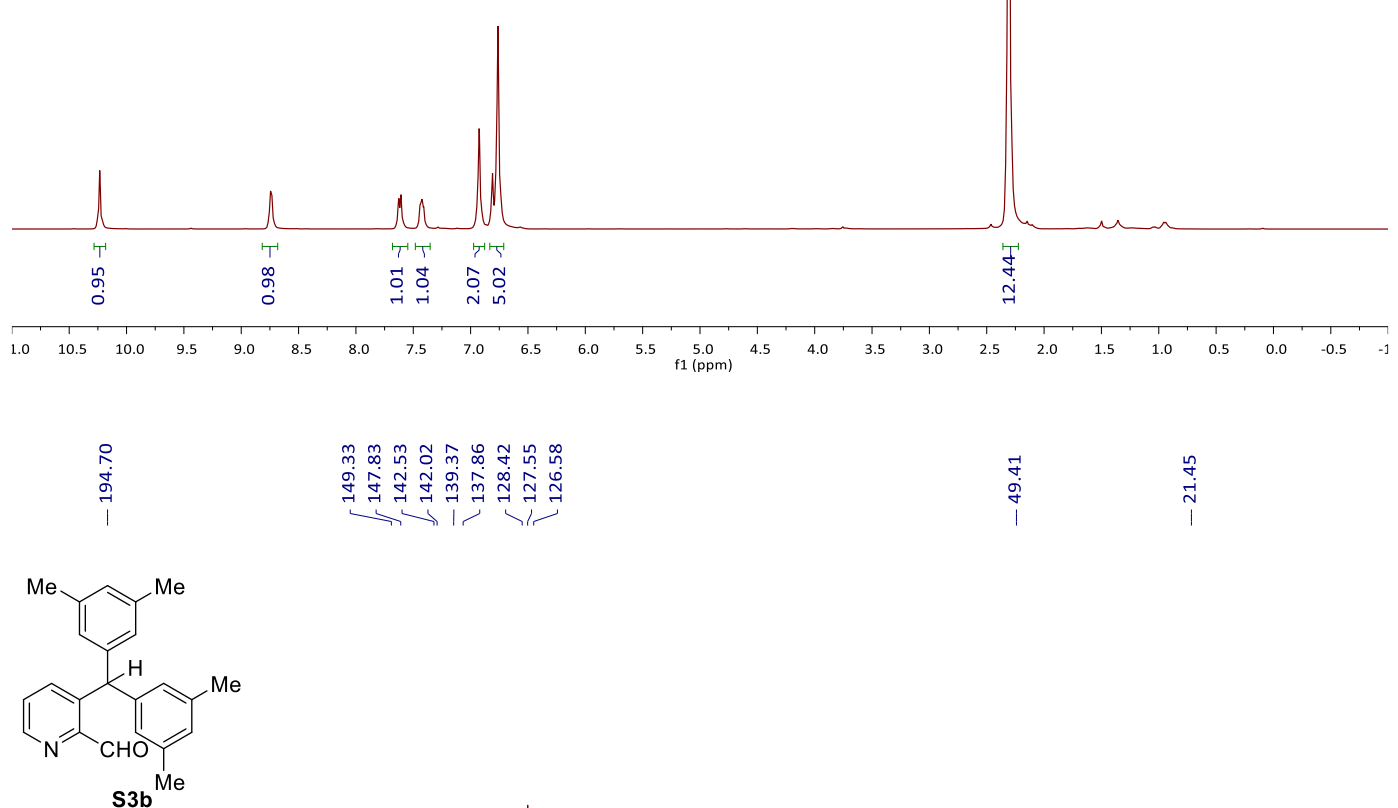

$\left({ }^{13} \mathrm{C}\right.$ NMR, $\left.101 \mathrm{MHz}, \mathrm{CDCl}_{3}\right)$

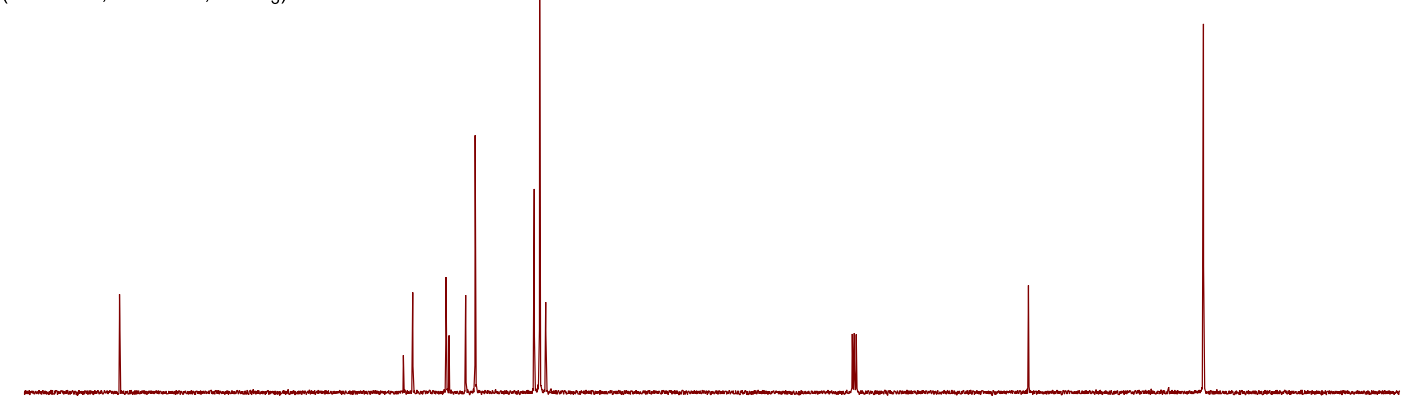

$10 \underset{\substack{100 \\ \mathrm{f}(1 \mathrm{pom})}}{1} 90$ 


\section{3-(Bis(3,5-diisopropylphenyl)methyl)picolinaldehyde (S3c)}
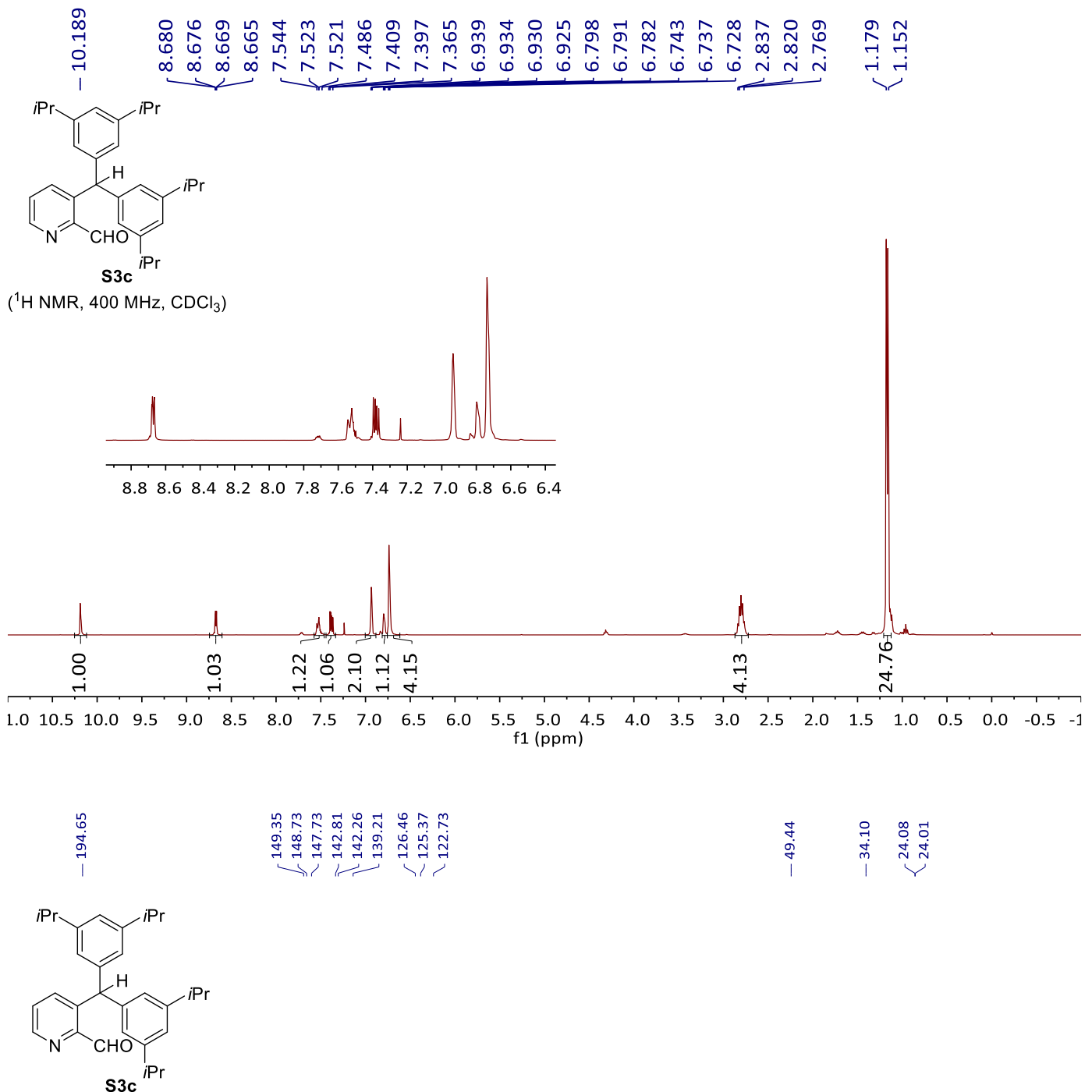

$\left({ }^{13} \mathrm{C} \mathrm{NMR,}, 101 \mathrm{MHz}, \mathrm{CDCl}_{3}\right)$

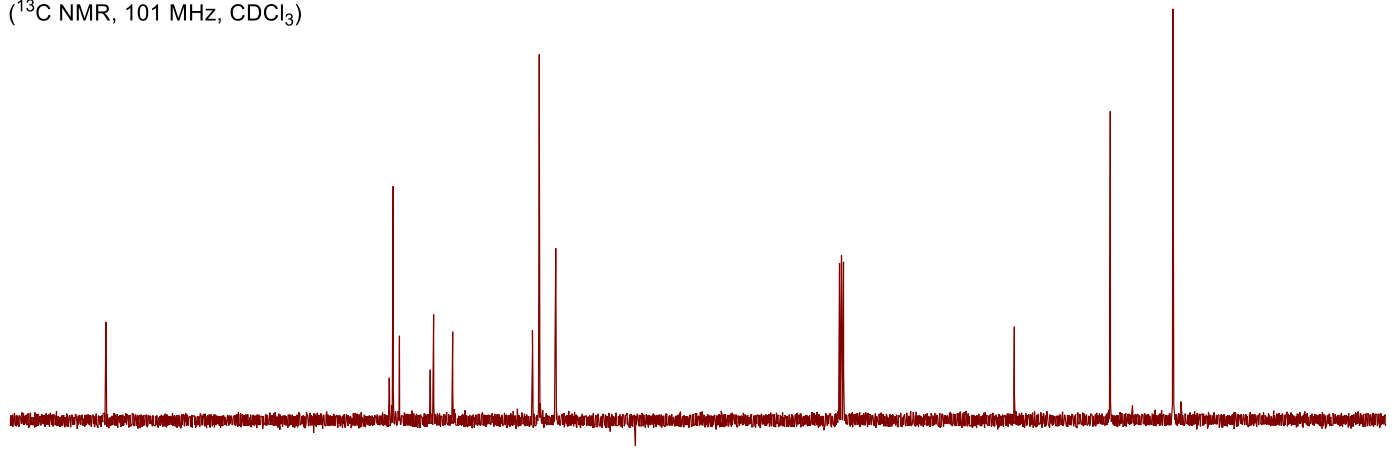

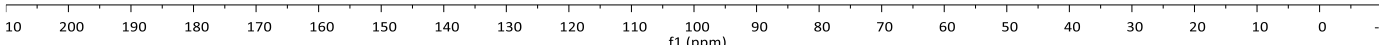


3-(Bis(3,5-di-tert-butylphenyl)methyl)picolinaldehyde (S3d)
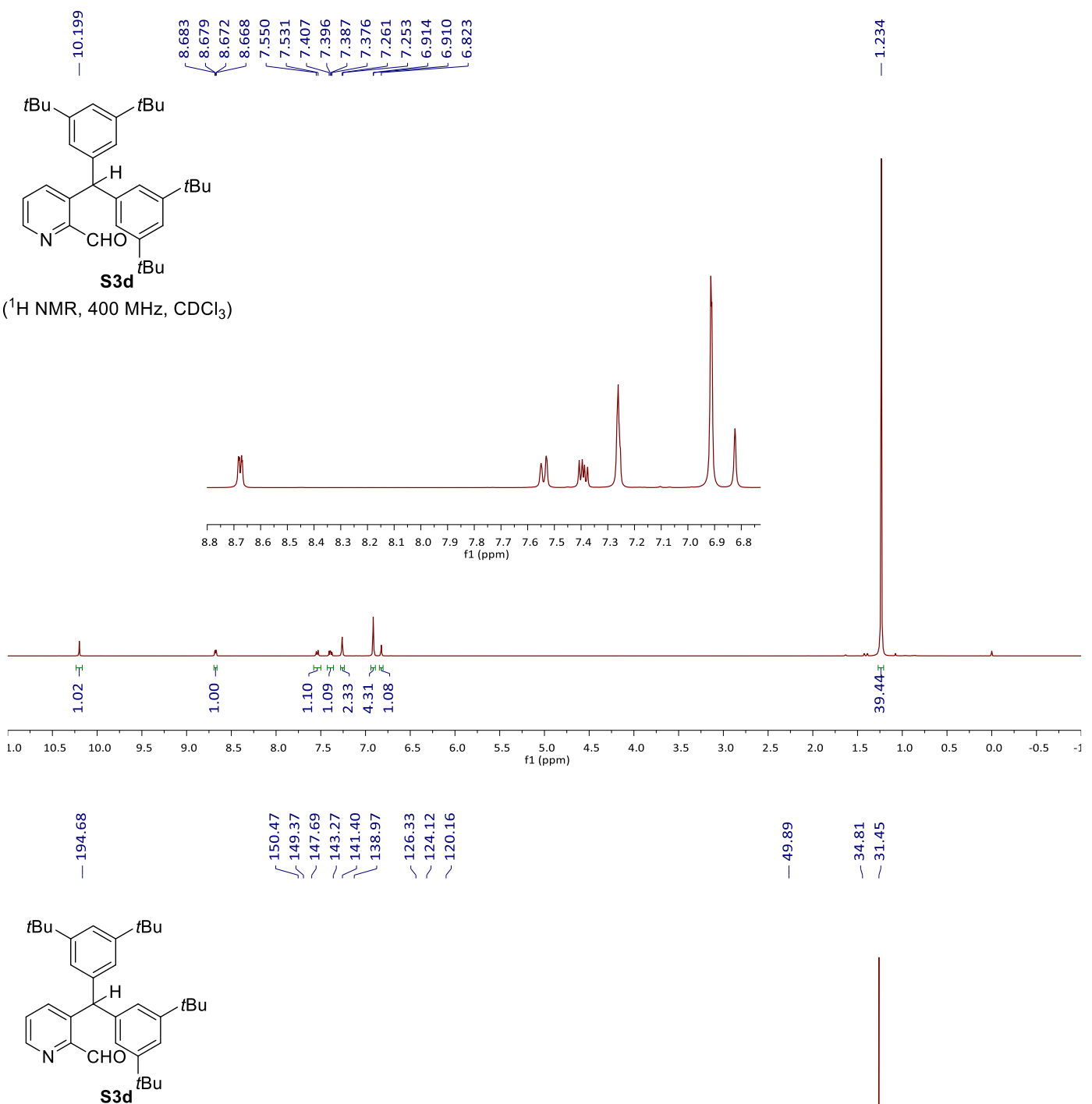

$\left({ }^{13} \mathrm{C} \mathrm{NMR}, 101 \mathrm{MHz}, \mathrm{CDCl}_{3}\right)$

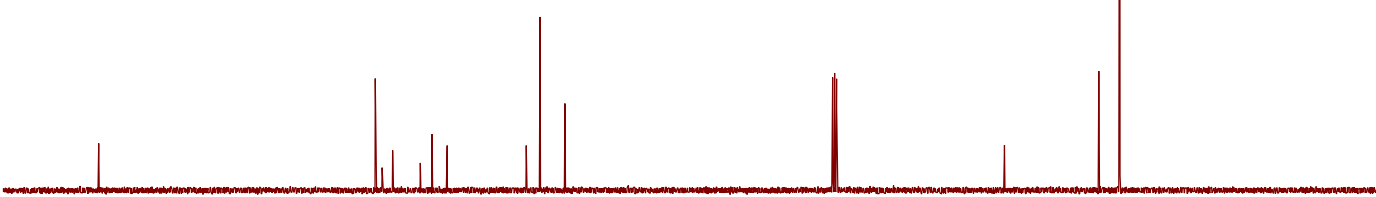

$\begin{array}{llllllllllllllllllllllllllllllllll}10 & 200 & 190 & 180 & 170 & 160 & 150 & 140 & 130 & 120 & 110 & 100 & 90 & 80 & 70 & 60 & 50 & 40 & 30 & 20 & 10 & 0\end{array}$ 


\section{Ligand (R)-S4a}

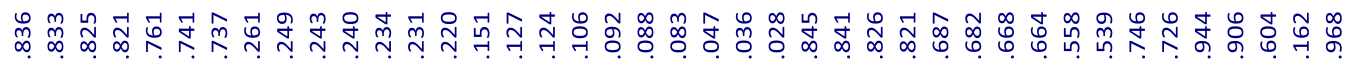
N

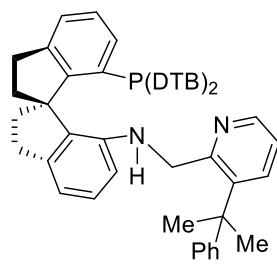

S4a

$\left({ }^{1} \mathrm{H} \mathrm{NMR}, 400 \mathrm{MHz}, \mathrm{CDCl}_{3}\right)$

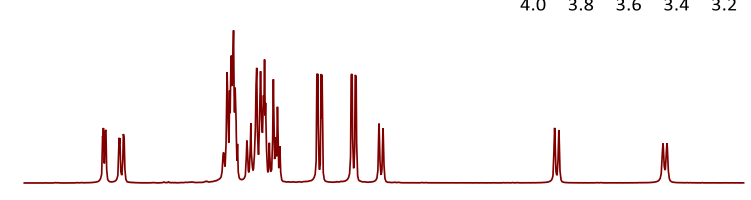

$\begin{array}{rrrrrrrrrrrrrrrrrrr}8.2 & 8.0 & 7.8 & 7.6 & 7.4 & 7.2 & 7.0 & 6.8 & 6.6 & 6.4 & 6.2 & 6.0 & 5.8 & 5.6 & 5.4 & 5.2 & 5.0\end{array}$

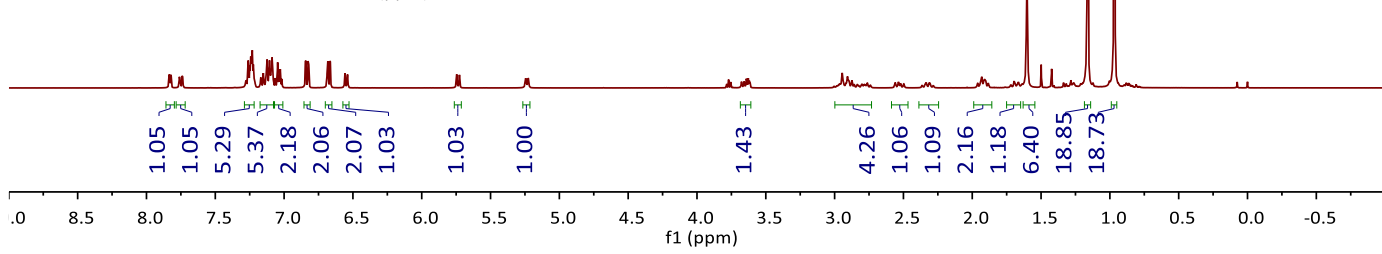

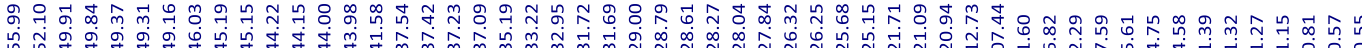

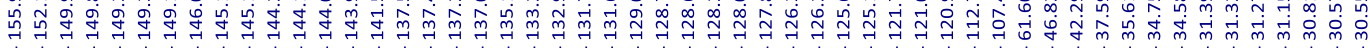

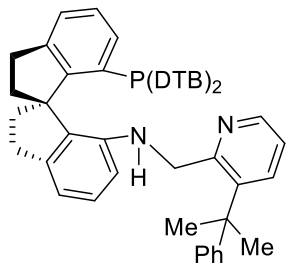

S4a

$\left({ }^{13} \mathrm{C}\right.$ NMR, $\left.101 \mathrm{MHz}, \mathrm{CDCl}_{3}\right)$

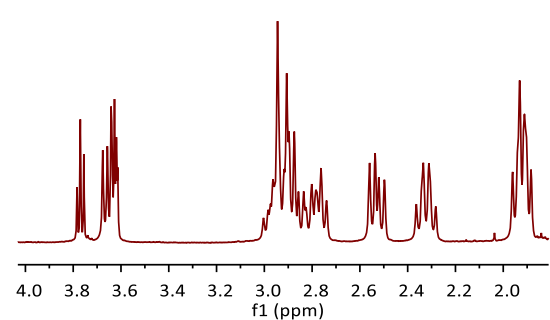

(ppm) 


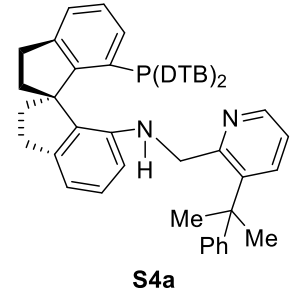

$\left({ }^{31} \mathrm{P}\right.$ NMR, $162 \mathrm{MHz}, \mathrm{CDCl}_{3}$ )

$\begin{array}{llllllllllllllllllllllllllllllllllll}00 & 90 & 80 & 70 & 60 & 50 & 40 & 30 & 20 & 10 & 0 & -10 & -20 & -30 & -40 & -50 & -60 & -70 & -80 & -90 & -100 & -110 & -120 & -130 & -140 & -150 & -160 & -170 & -180 & -190 & -2\end{array}$

\section{Ligand (R)-S4b}

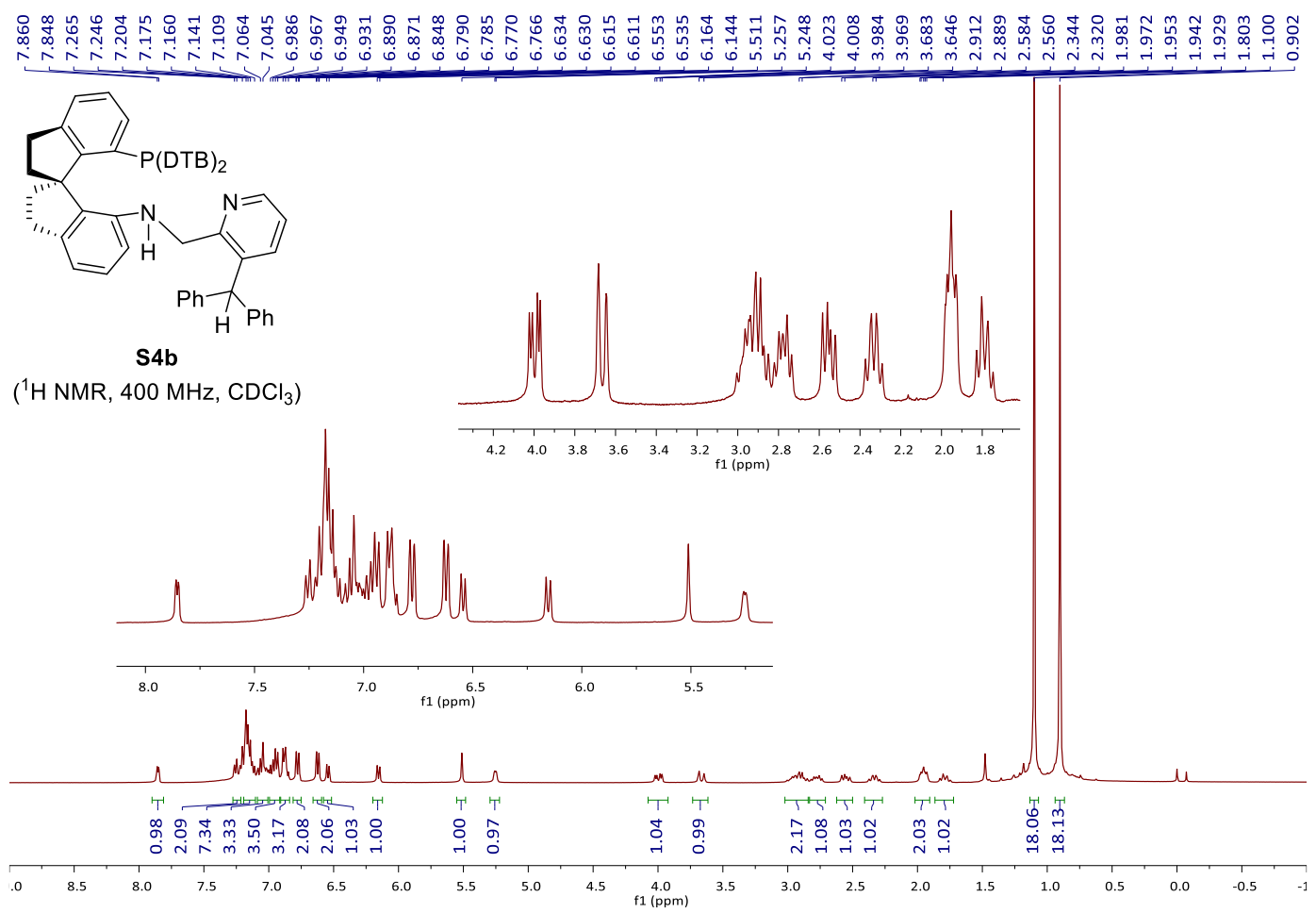




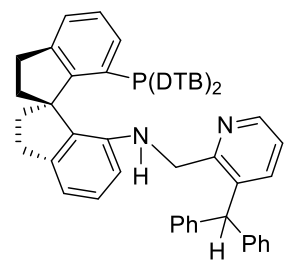

S4b

$\left({ }^{13} \mathrm{C} \mathrm{NMR}, 101 \mathrm{MHz}, \mathrm{CDCl}_{3}\right)$
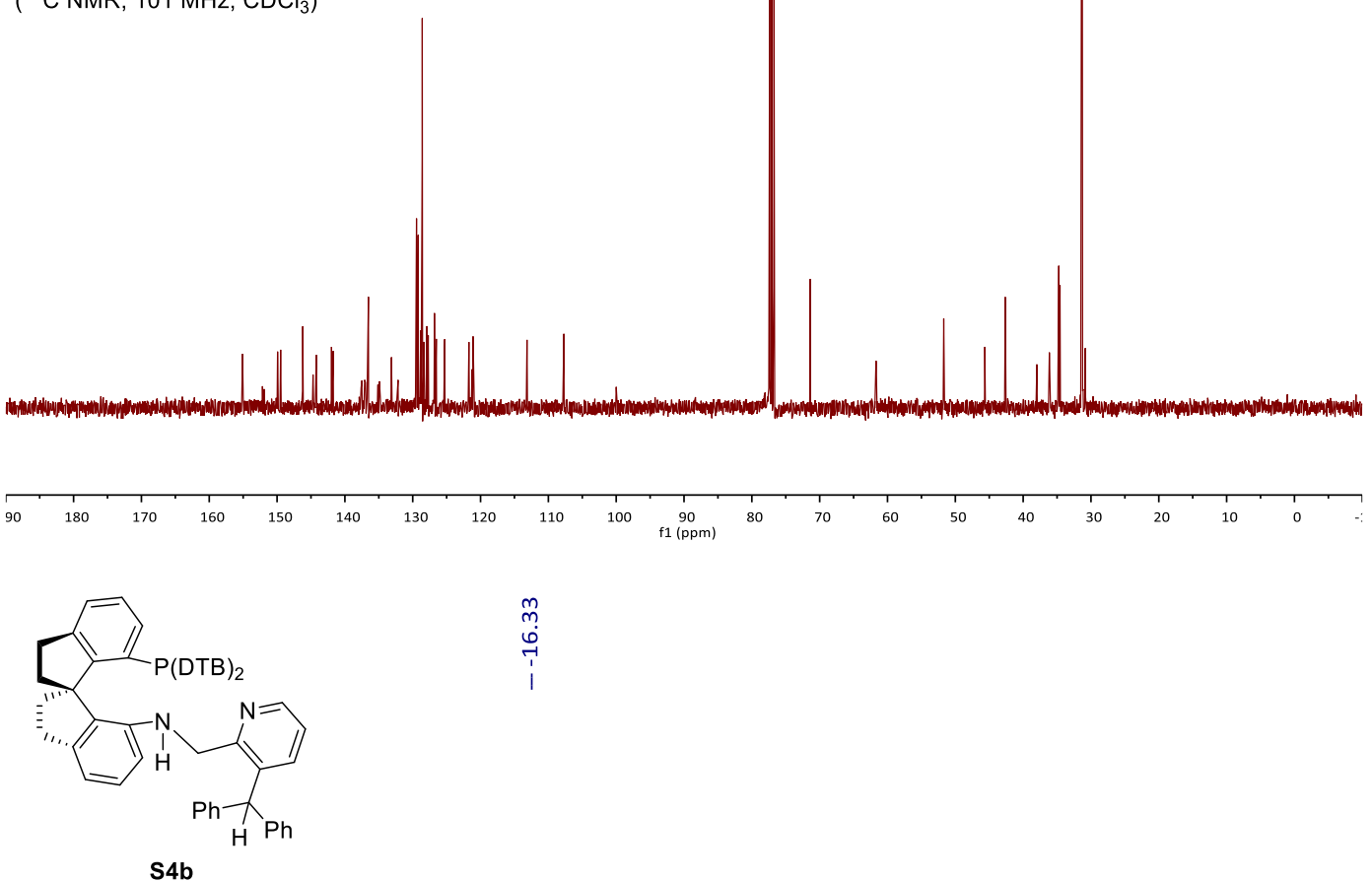

$n$
$m$
0
$\vdots$
1

$\left({ }^{31} \mathrm{P}\right.$ NMR, $\left.162 \mathrm{MHz}, \mathrm{CDCl}_{3}\right)$

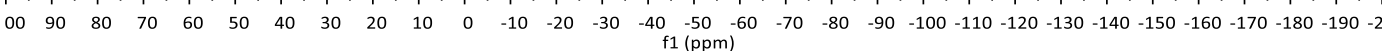




\section{Ligand (R)-S4c}

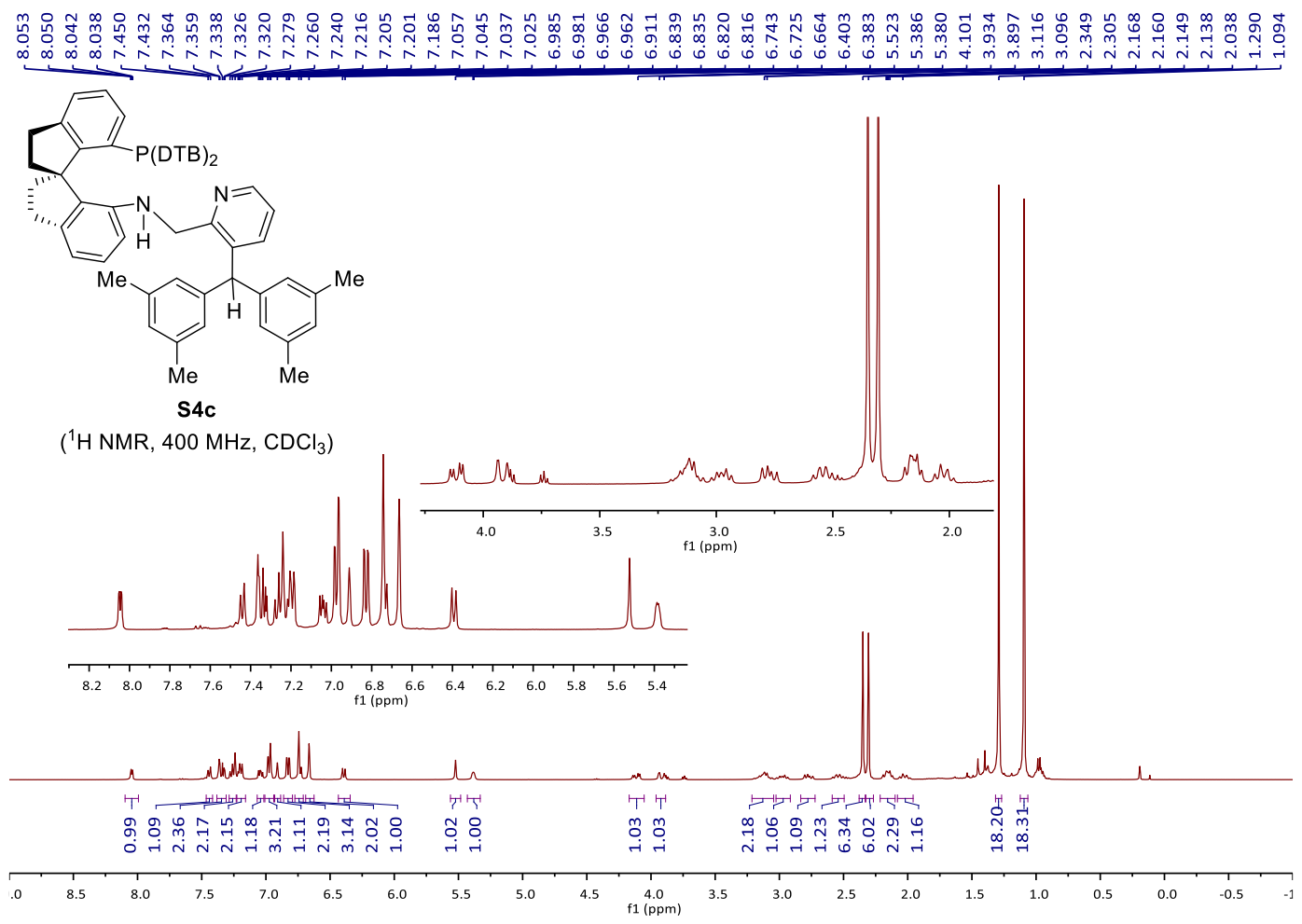

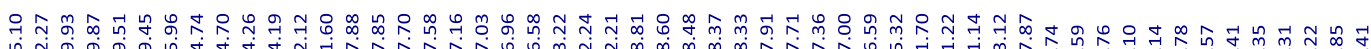

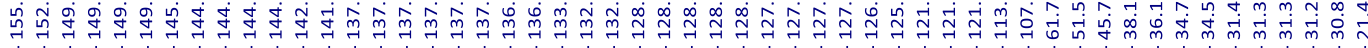

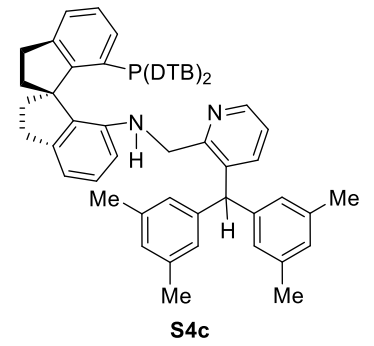

$\left({ }^{13} \mathrm{C} \mathrm{NMR}, 101 \mathrm{MHz}, \mathrm{CDCl}_{3}\right)$

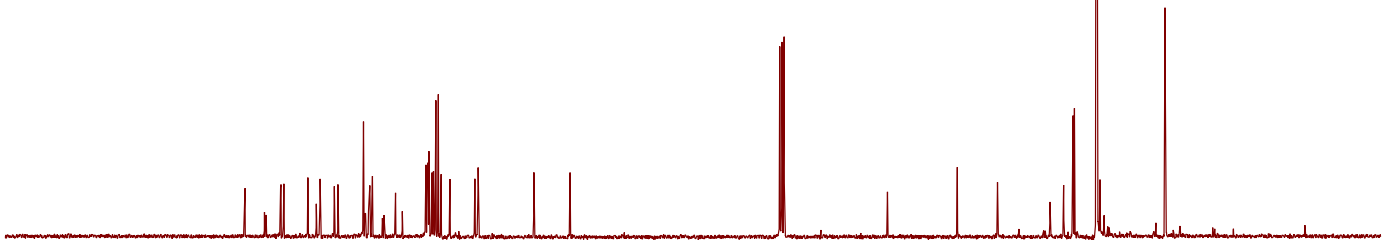

\begin{tabular}{lllllllllllllllllllllll}
\hline 90 & 180 & 170 & 160 & 150 & 140 & 130 & 120 & 110 & 100 & 90 & 80 & 70 & 60 & 50 & 40 & 30 & 20 & 10 & 0 & -3
\end{tabular} 


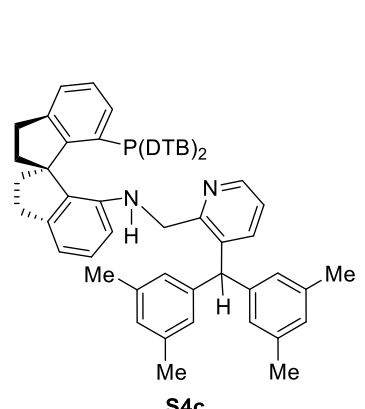

$\left({ }^{31} \mathrm{P}\right.$ NMR, $\left.162 \mathrm{MHz}, \mathrm{CDCl}_{3}\right)$

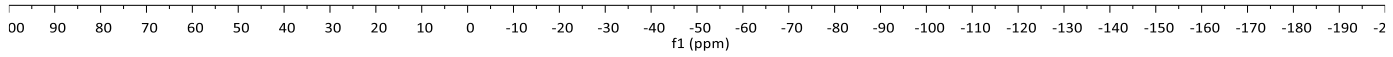

\section{Ligand (R)-S4d}

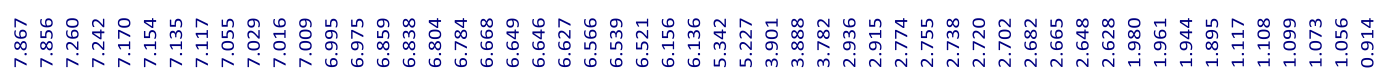

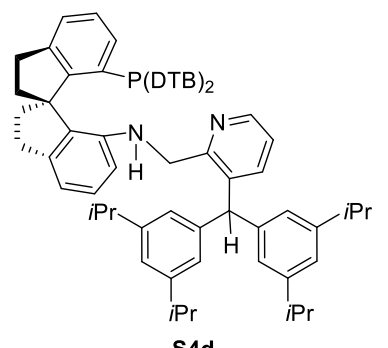

$\left({ }^{1} \mathrm{H}\right.$ NMR, $\left.400 \mathrm{MHz}, \mathrm{CDCl}_{3}\right)$
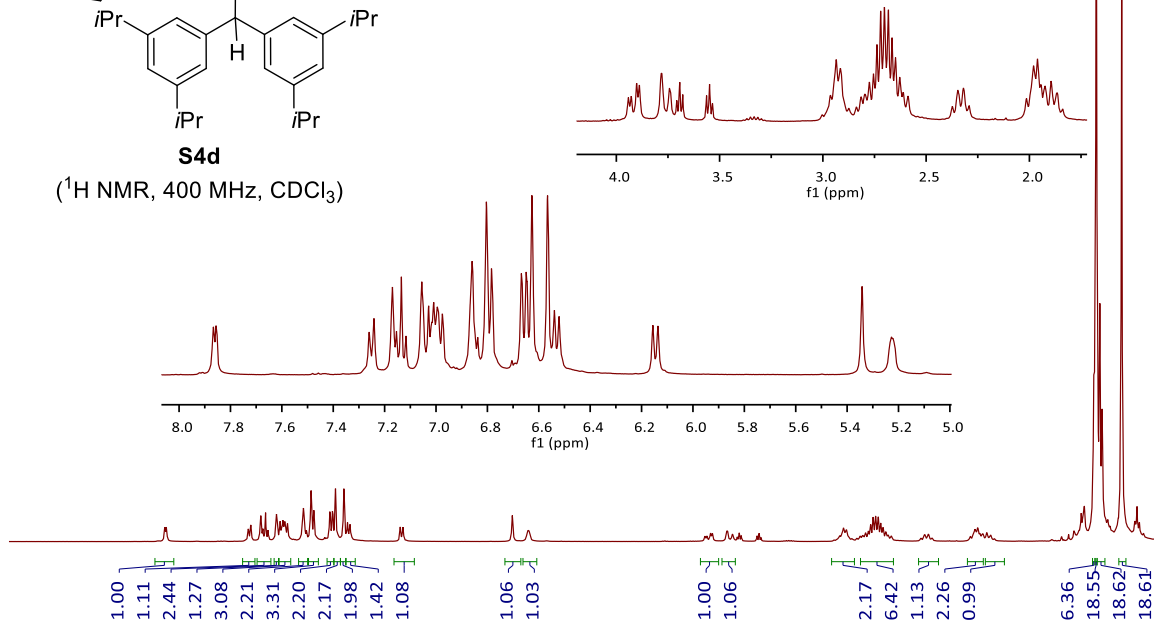

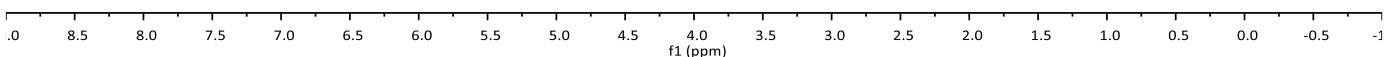




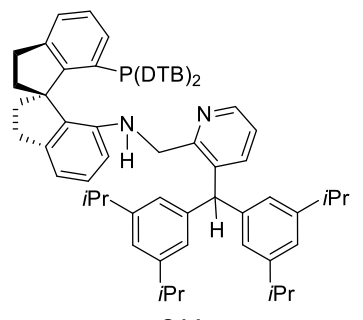

$\left({ }^{13} \mathrm{C} \mathrm{NMR}, 101 \mathrm{MHz}, \mathrm{CDCl}_{3}\right)$
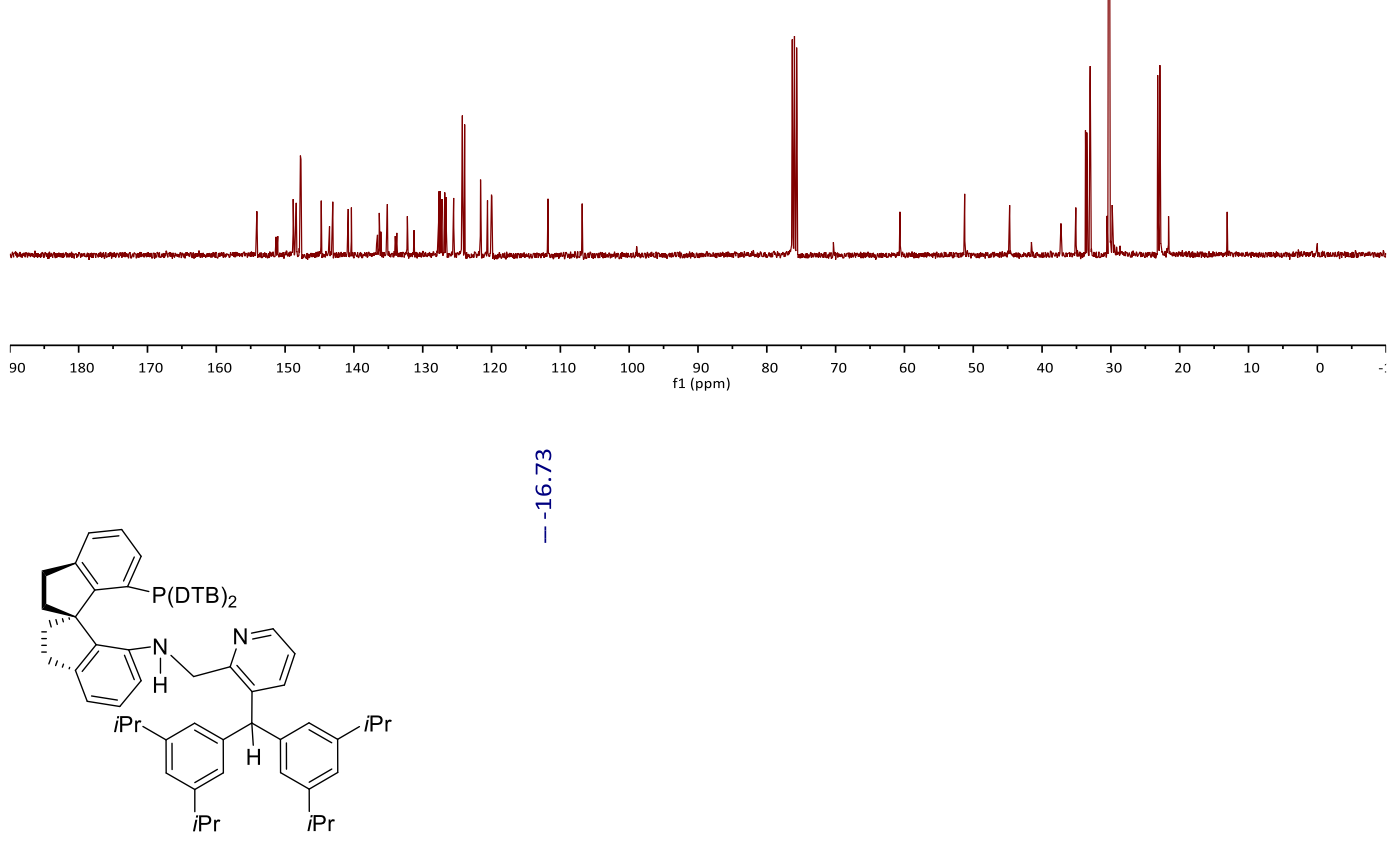

\section{$n$
$\stackrel{n}{0}$
$\stackrel{1}{1}$
1}

( ${ }^{31} \mathrm{P} \mathrm{NMR}, 162 \mathrm{MHz}, \mathrm{CDCl}_{3}$ )

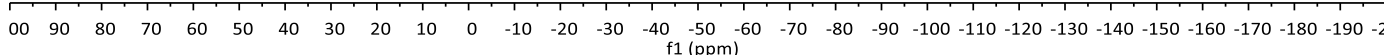




\section{Ligand (R)-S4e}

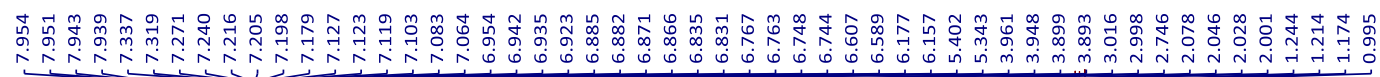

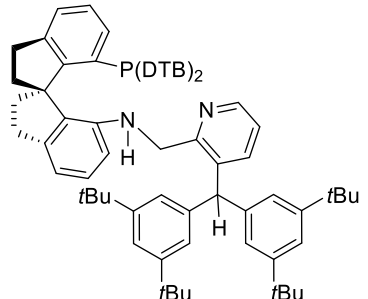

S4e

$\left({ }^{1} \mathrm{H} \mathrm{NMR}, 400 \mathrm{MHz}, \mathrm{CDCl}_{3}\right)$

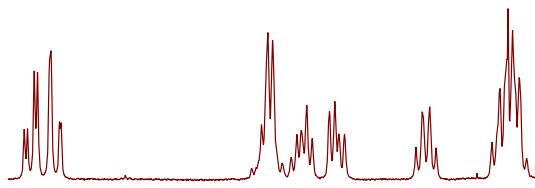

$\begin{array}{lllllllllllll}4.0 & 3.8 & 3.6 & 3.4 & 3.2 & \begin{array}{c}3.0 \\ \mathrm{f}(\mathrm{ppm})\end{array} & 2.8 & 2.6 & 2.4 & 2.2 & 2.0\end{array}$

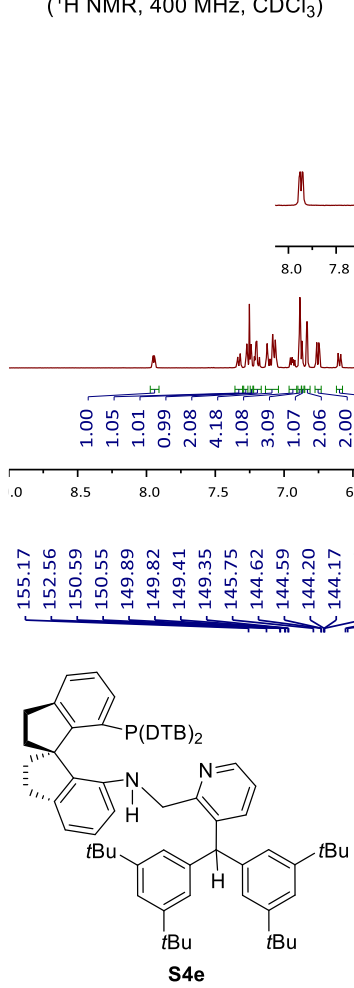

$\left({ }^{13} \mathrm{C} \mathrm{NMR}, 101 \mathrm{MHz}, \mathrm{CDCl}_{3}\right)$

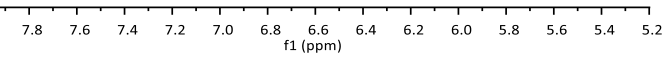

mulle
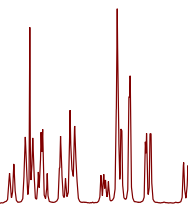

(a) 


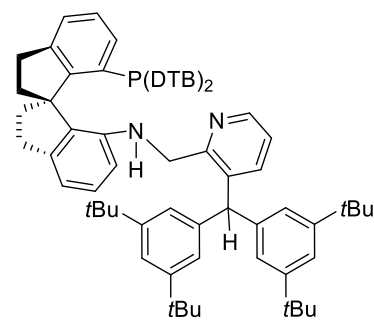

ì

S4e

( ${ }^{31} \mathrm{P} \mathrm{NMR}, 162 \mathrm{MHz}, \mathrm{CDCl}_{3}$ )

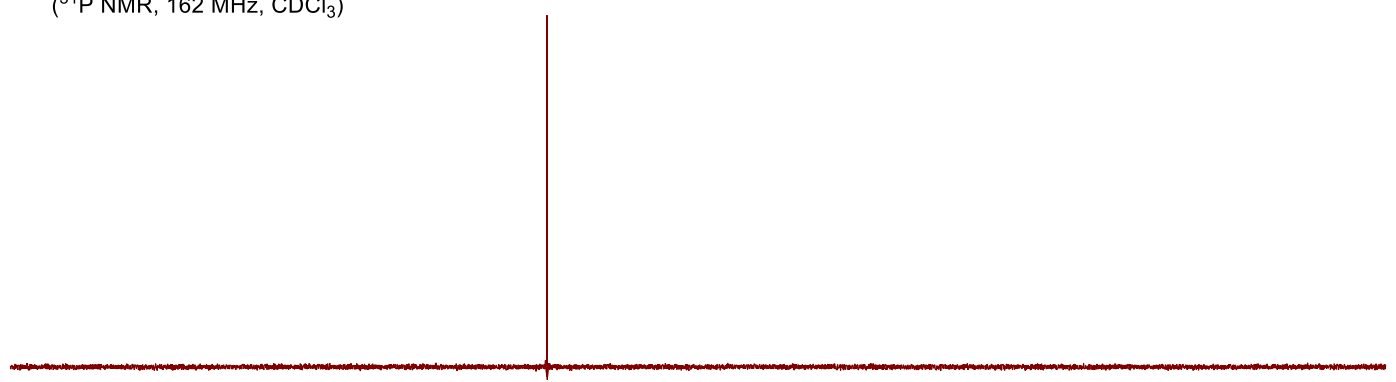

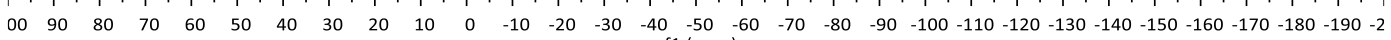


Diethyl-2-(1-(tosyloxy)ethylidene)malonate (S6a)

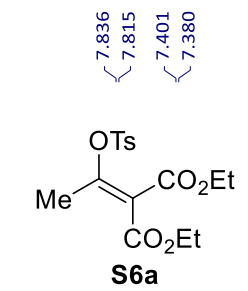

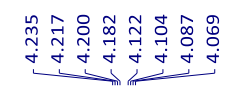

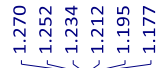

$\left({ }^{1} \mathrm{H}\right.$ NMR, $\left.400 \mathrm{MHz}, \mathrm{CDCl}_{3}\right)$
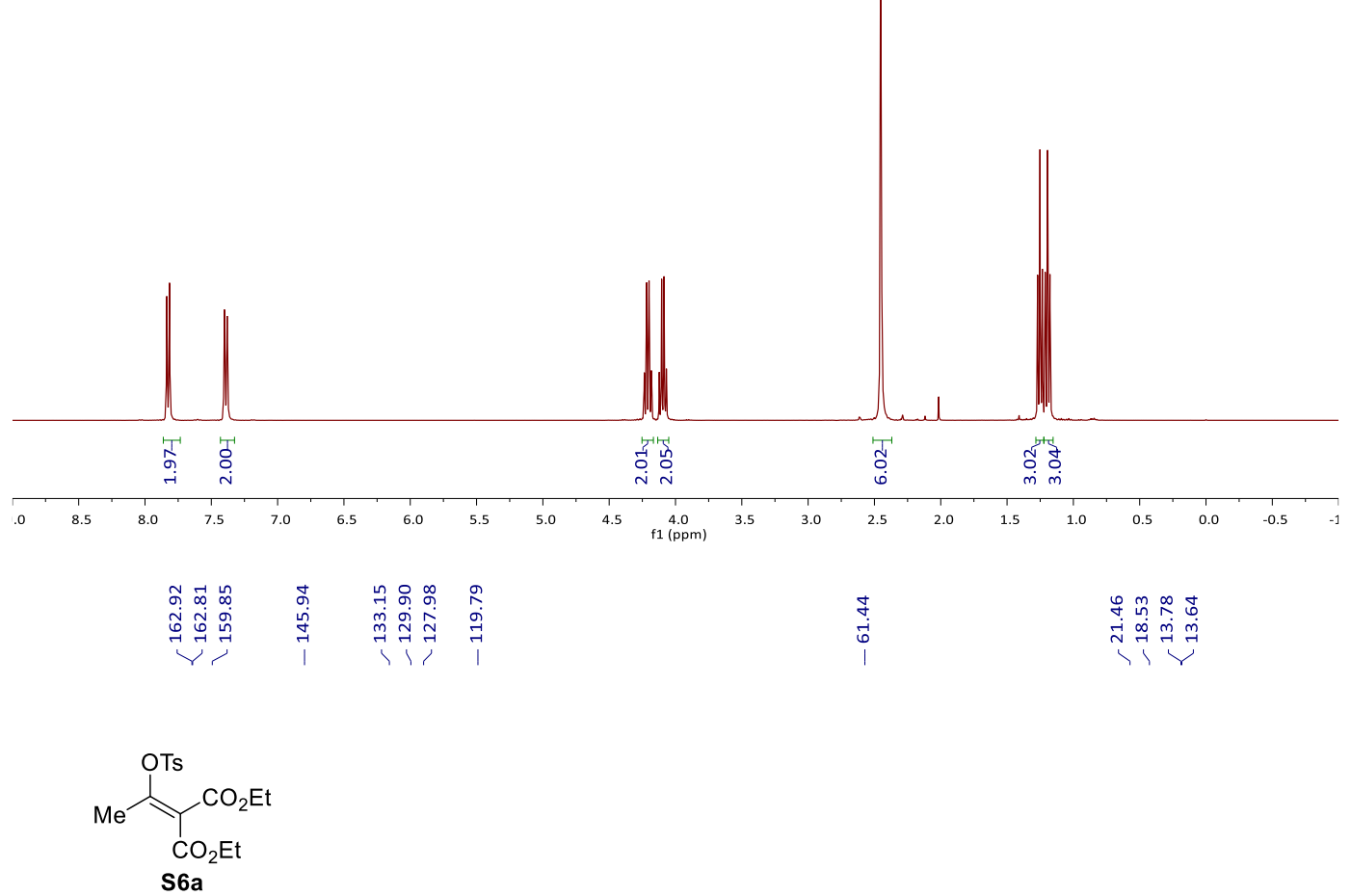

$\left({ }^{13} \mathrm{C} \mathrm{NMR}, 101 \mathrm{MHz}, \mathrm{CDCl}_{3}\right)$

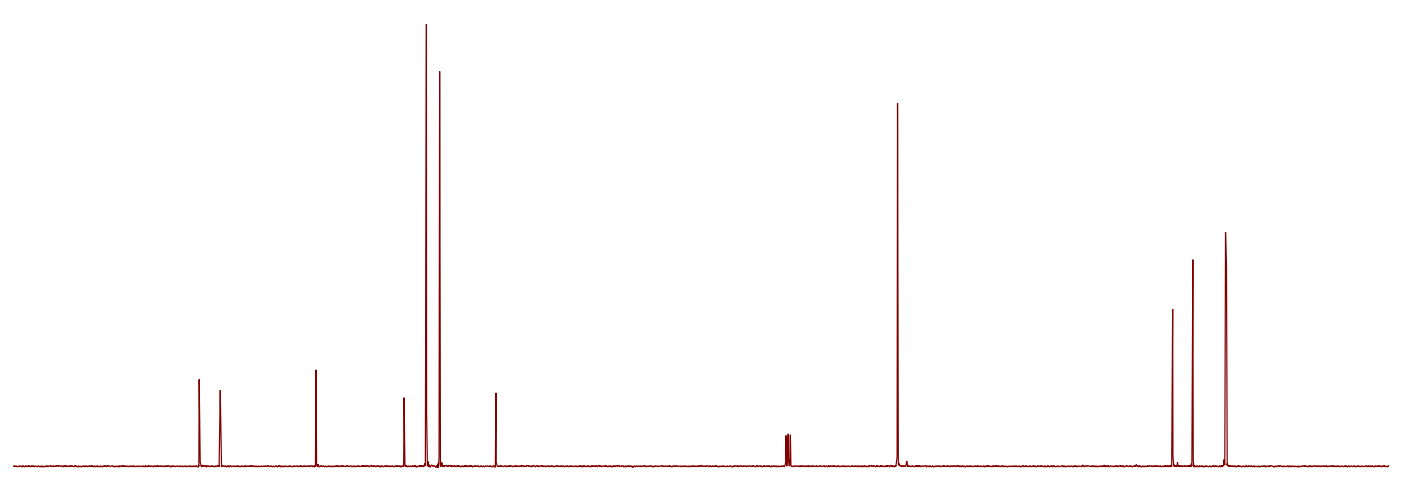

\begin{tabular}{lllllllllllllllllllllll}
\hline & 180 & 170 & 160 & 150 & 140 & 130 & 120 & 110 & 100 & 90 & 80 & 70 & 60 & 50 & 40 & 30 & 20 & 10 & 0 & $-:$
\end{tabular} 
Diethyl-2-(1-(tosyloxy)propylidene)malonate (S6b)

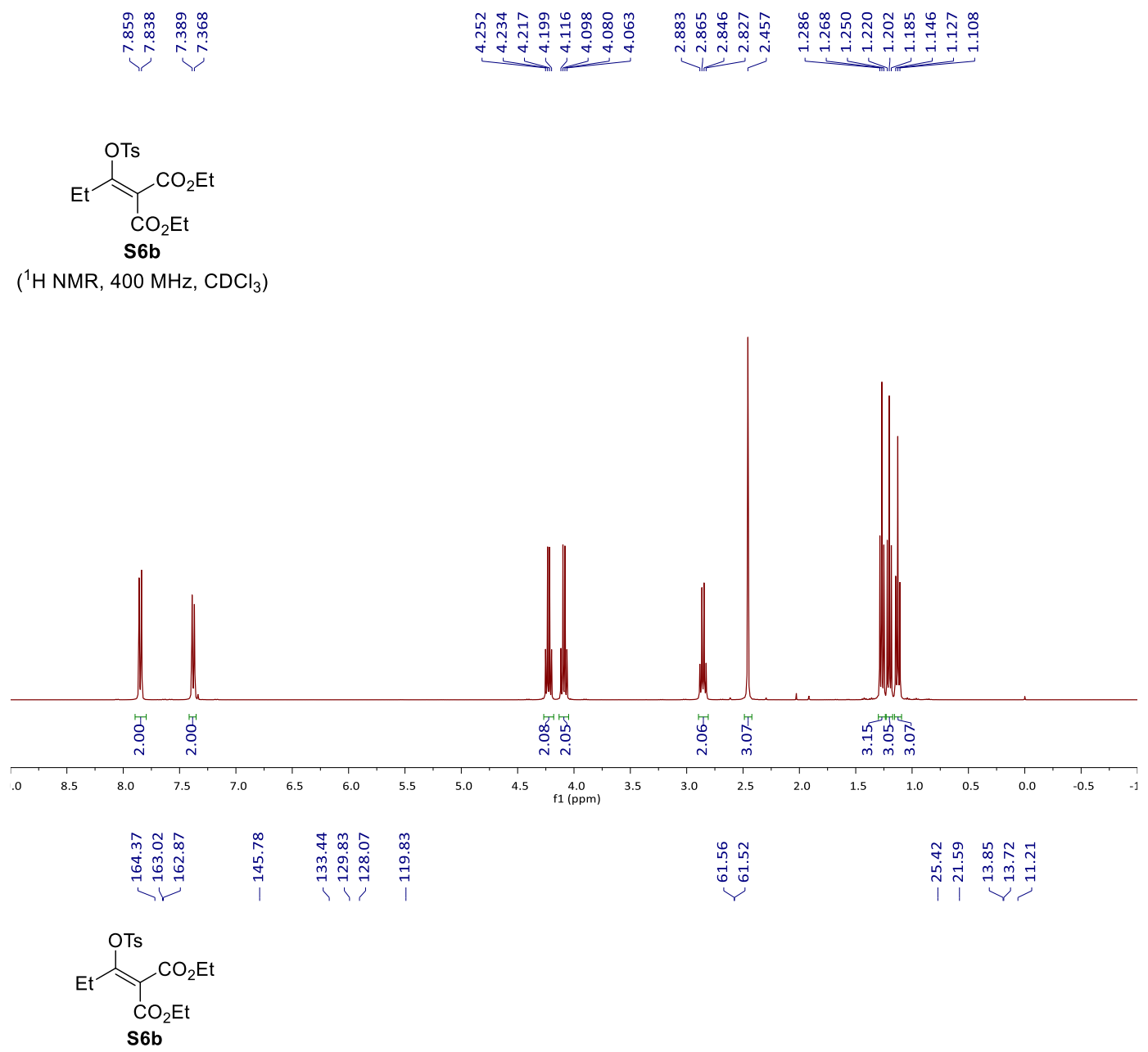

$\left({ }^{13} \mathrm{C} \mathrm{NMR}, 101 \mathrm{MHz}, \mathrm{CDCl}_{3}\right)$

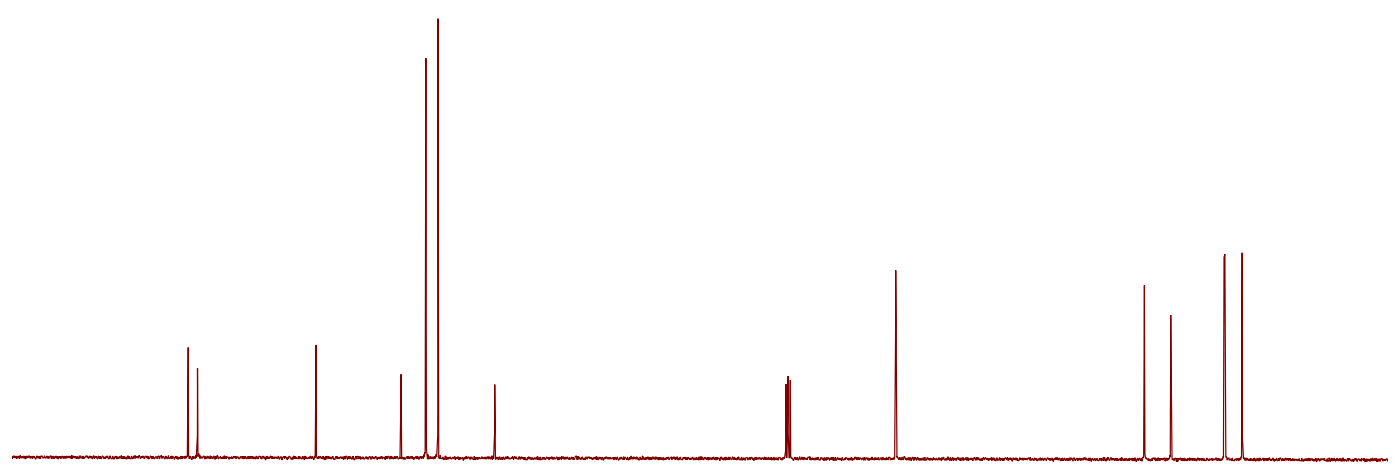

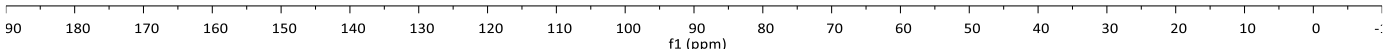


Diethyl-2-(1-(tosyloxy)butylidene)malonate (S6c)

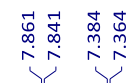

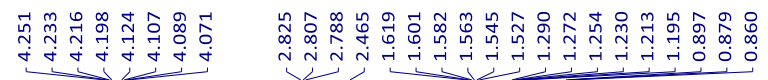

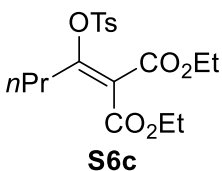

$\left({ }^{1} \mathrm{H} \mathrm{NMR}, 400 \mathrm{MHz}, \mathrm{CDCl}_{3}\right)$

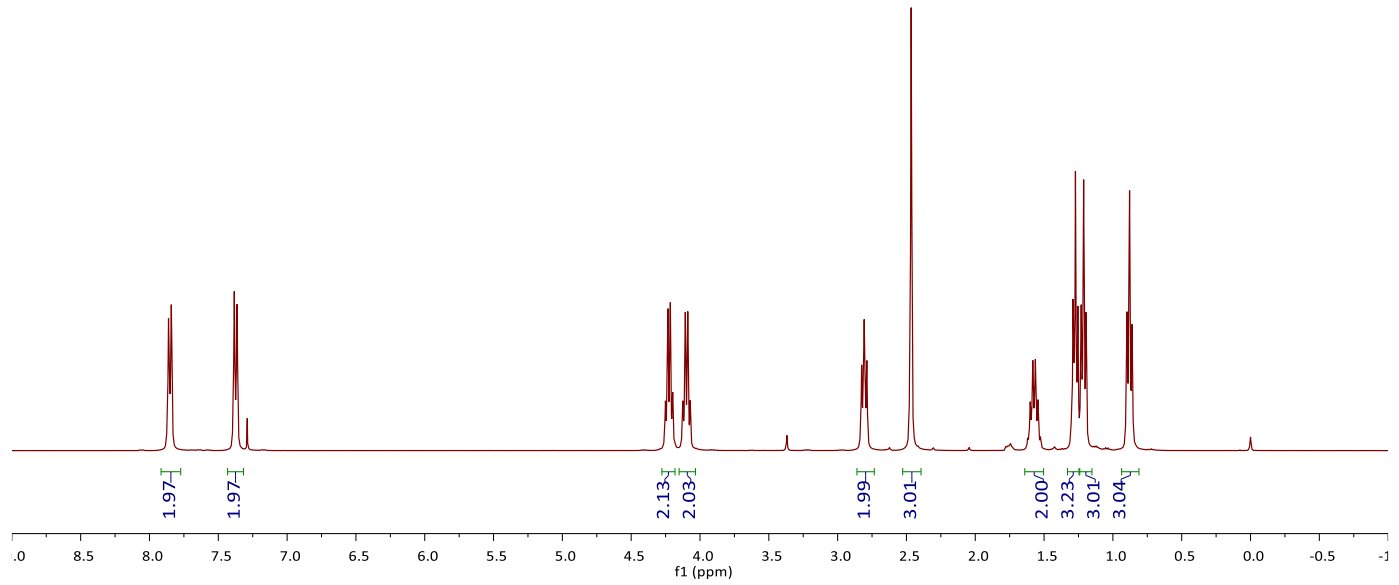

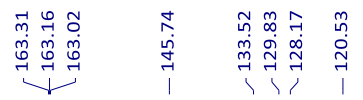

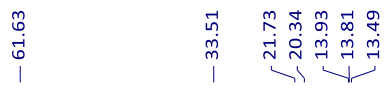

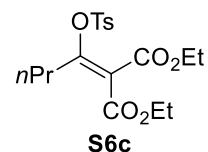

$\left({ }^{13} \mathrm{C}\right.$ NMR, $\left.101 \mathrm{MHz}, \mathrm{CDCl}_{3}\right)$

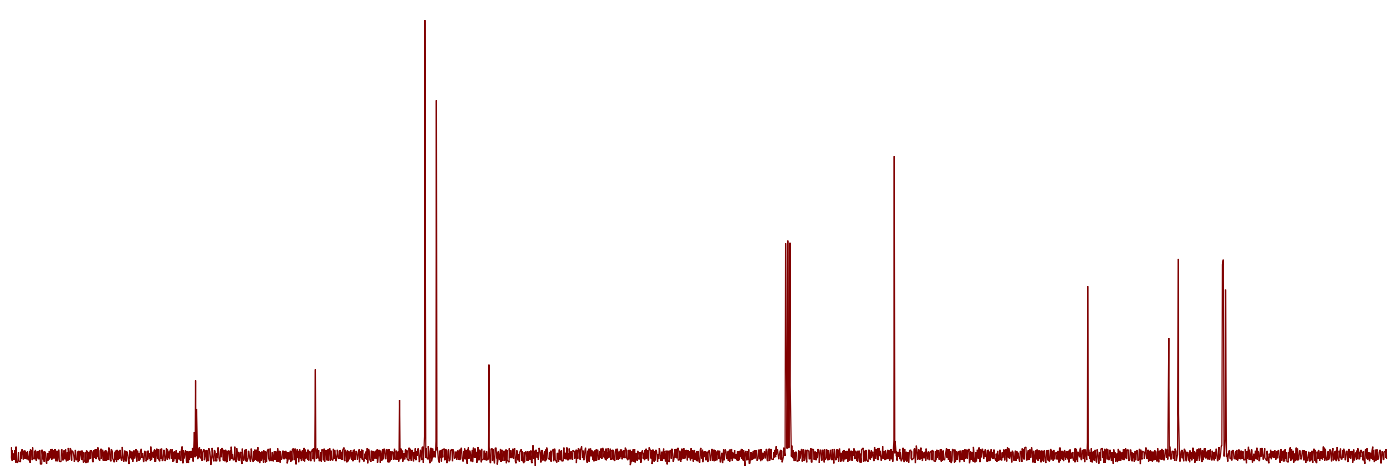

\begin{tabular}{lllllllllllllllllllllllll}
\hline 90 & 180 & 170 & 160 & 150 & 140 & 130 & 120 & 110 & 100 & 90 & 80 & 70 & 60 & 50 & 40 & 30 & 20 & 10 & 0 & $-:$
\end{tabular} 
Diethyl 2-(1-phenylethylidene)malonate (2a)

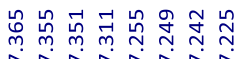

$\operatorname{cich}$

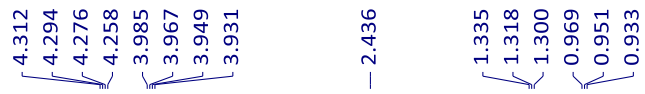

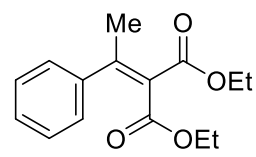

2a

$\left({ }^{1} \mathrm{H} \mathrm{NMR}, 400 \mathrm{MHz}, \mathrm{CDCl}_{3}\right)$

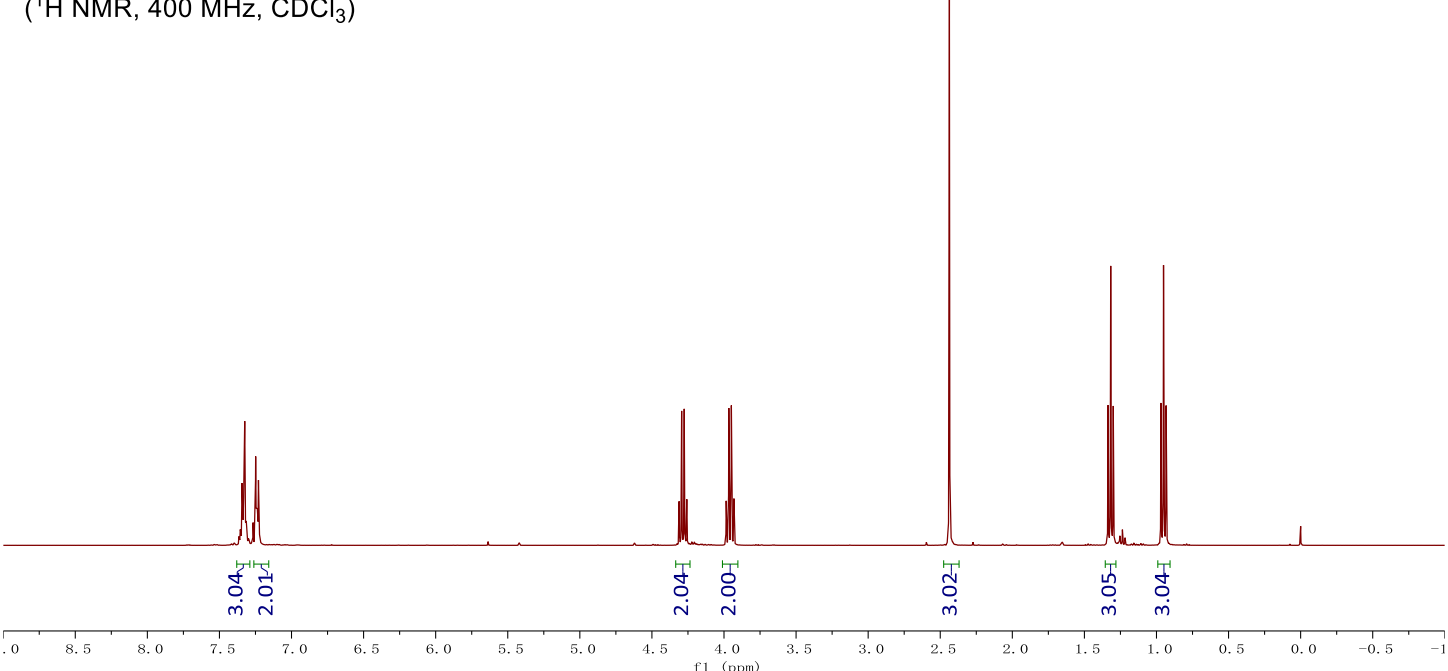

Diethyl 2-(1-phenylpropylidene)malonate (2b)
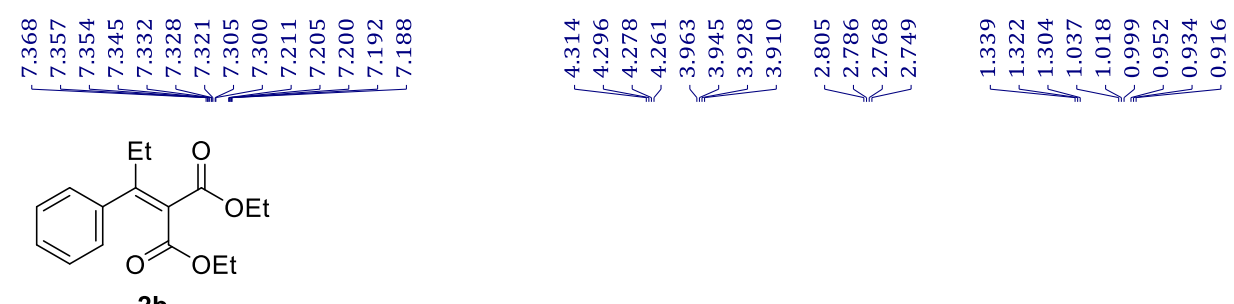

$\left({ }^{1} \mathrm{H} \mathrm{NMR}, 400 \mathrm{MHz}, \mathrm{CDCl}_{3}\right)$

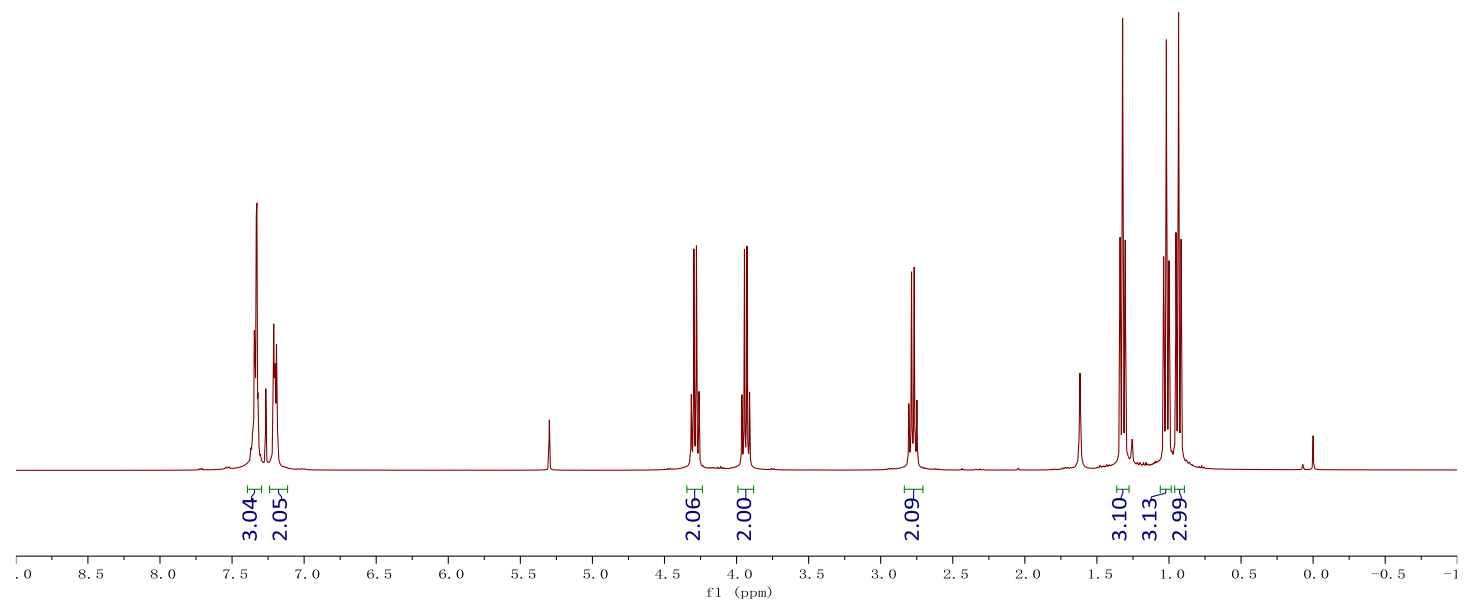


Diethyl-2-(1-phenylbutylidene)malonate (2c)

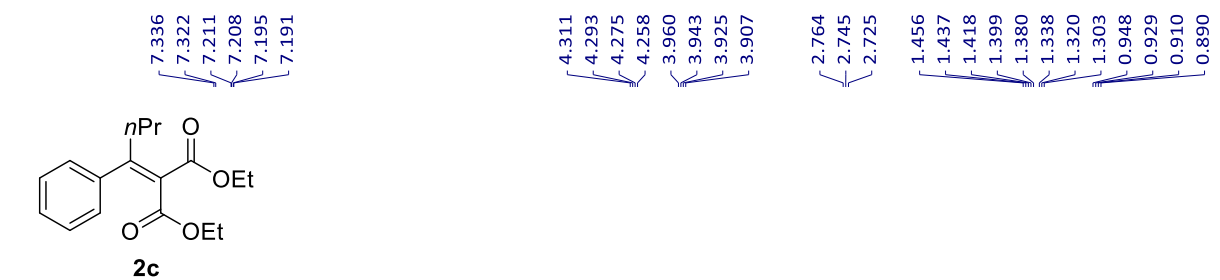

$\left({ }^{1} \mathrm{H}\right.$ NMR, $\left.400 \mathrm{MHz}, \mathrm{CDCl}_{3}\right)$

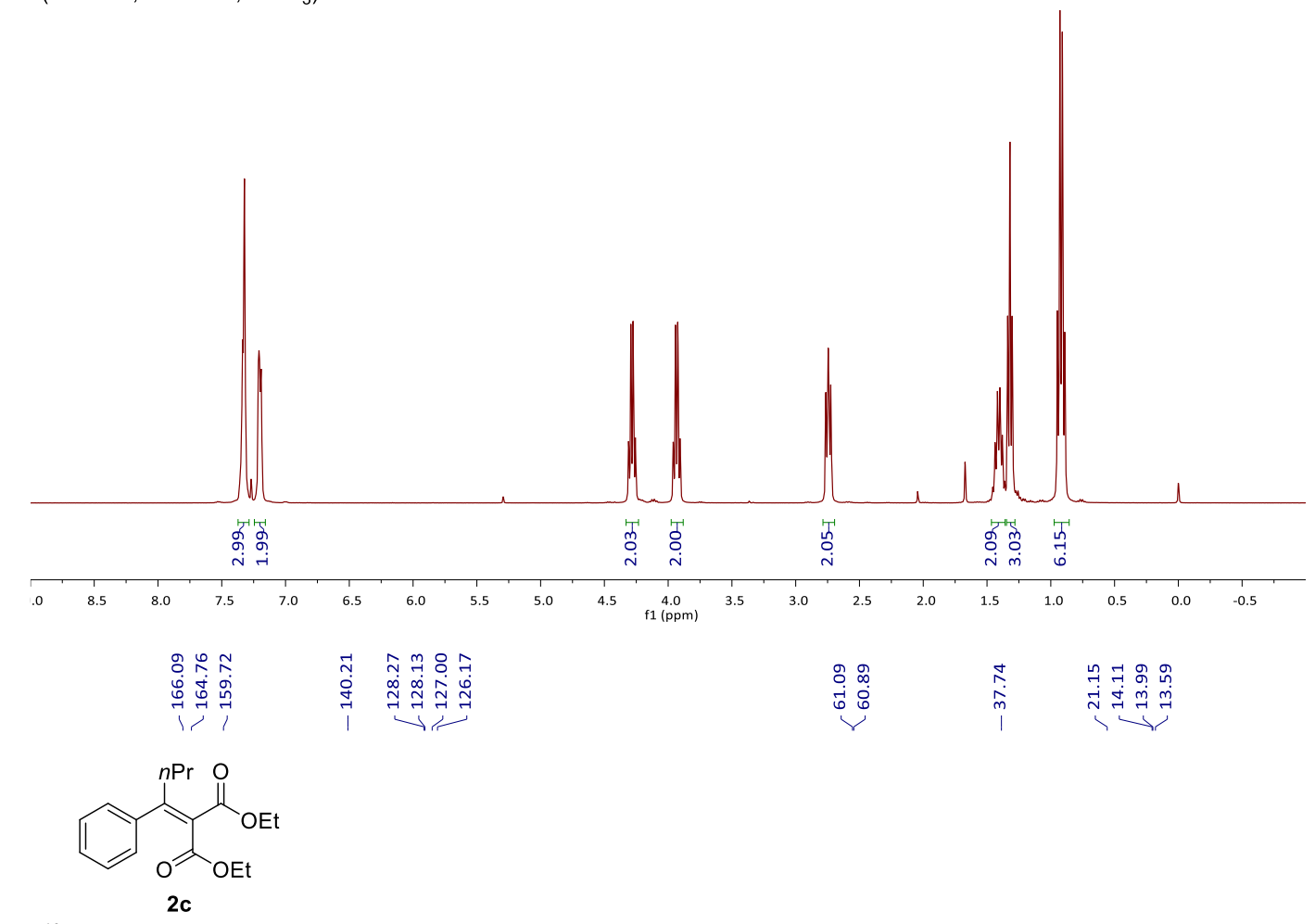

$\left({ }^{13} \mathrm{C}\right.$ NMR, $\left.101 \mathrm{MHz}, \mathrm{CDCl}_{3}\right)$

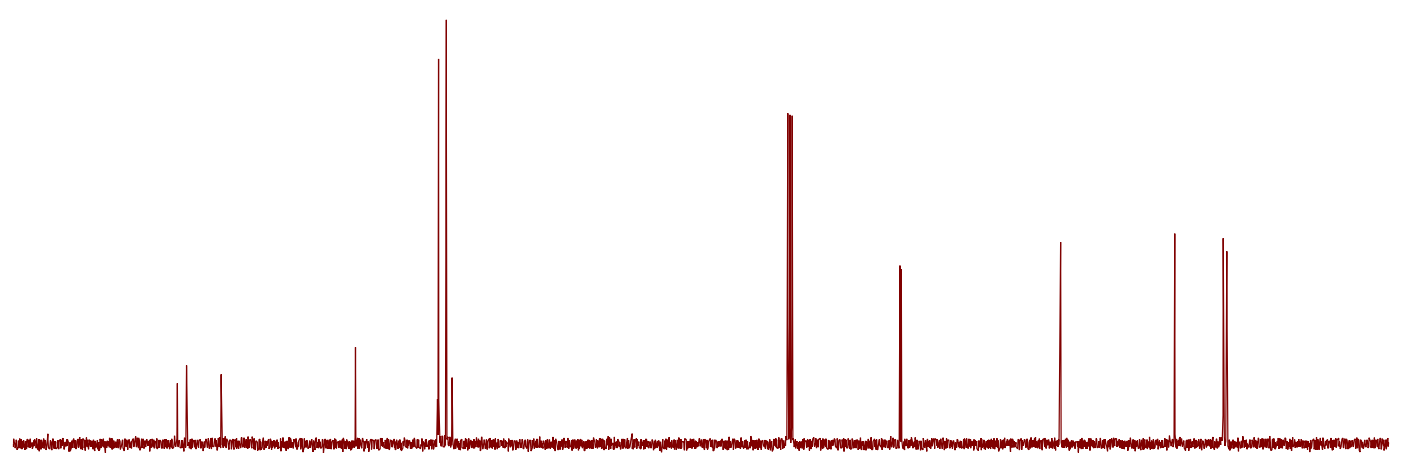

\begin{tabular}{lllllllllllllllllllllllll}
\hline 90 & 180 & 170 & 160 & 150 & 140 & 130 & 120 & 110 & 100 & 90 & 80 & 70 & 60 & 50 & 40 & 30 & 20 & 10 & 0 & $-:$
\end{tabular} 
Diethyl-2-(1-(4-fluorophenyl)ethylidene)malonate (2d)

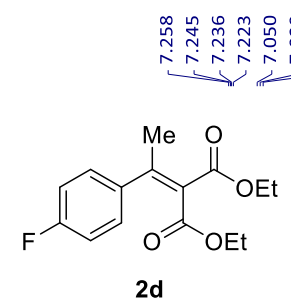

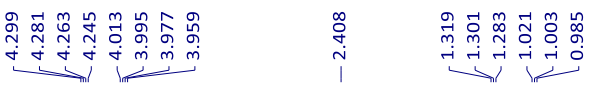

$\left({ }^{1} \mathrm{H} \mathrm{NMR}, 400 \mathrm{MHz}, \mathrm{CDCl}_{3}\right)$

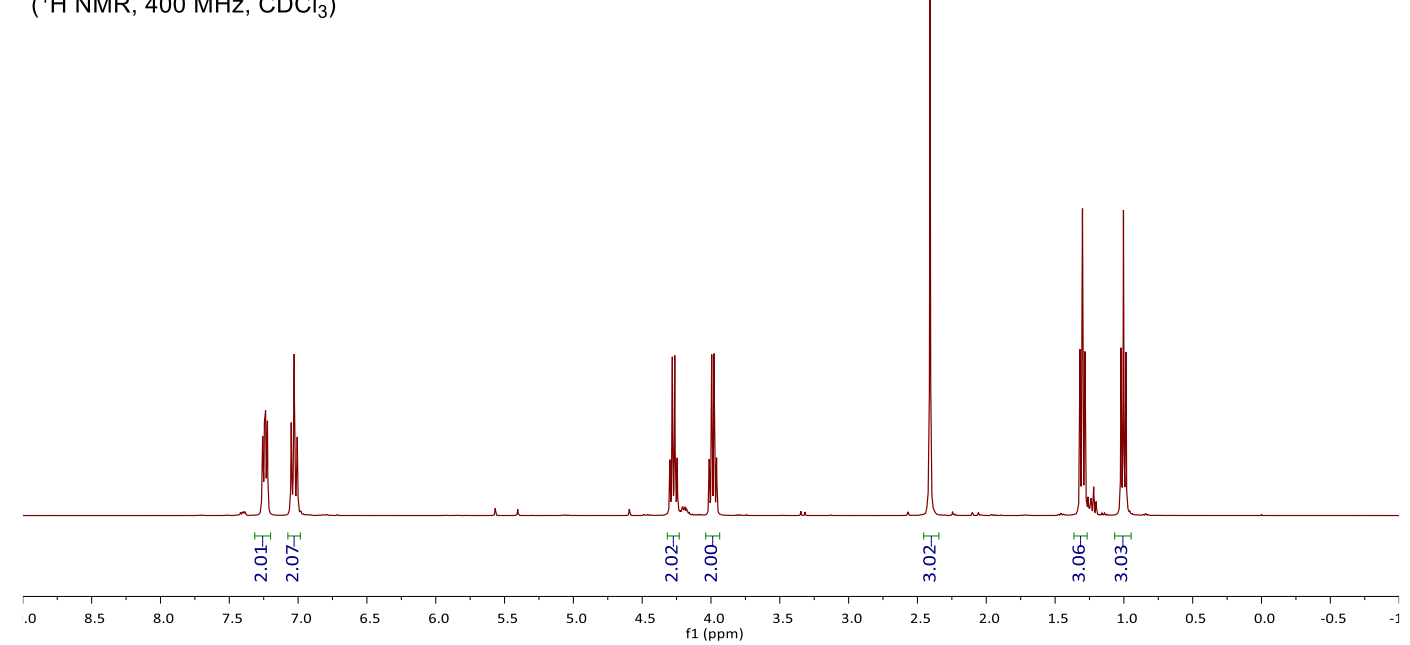

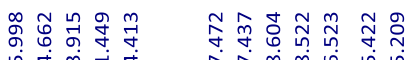

岕过自它点

$\stackrel{0}{\stackrel{2}{7}}$

تું

ஓ

^

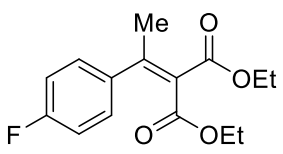

$\left({ }^{13} \mathrm{C} \mathrm{NMR}, 101 \mathrm{MHz}, \mathrm{CDCl}_{3}\right)$

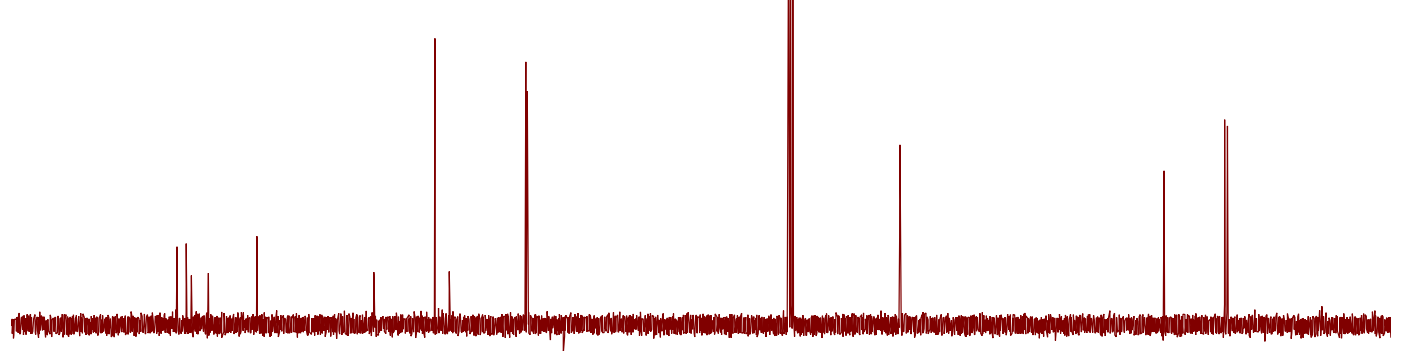


Diethyl-2-(1-(4-chlorophenyl)ethylidene)malonate (2e)

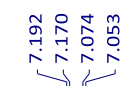

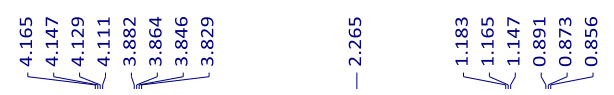

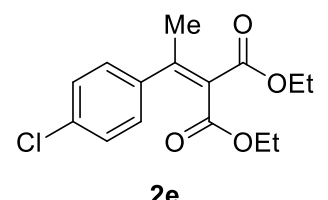

$\left({ }^{1} \mathrm{H}\right.$ NMR, $\left.400 \mathrm{MHz}, \mathrm{CDCl}_{3}\right)$
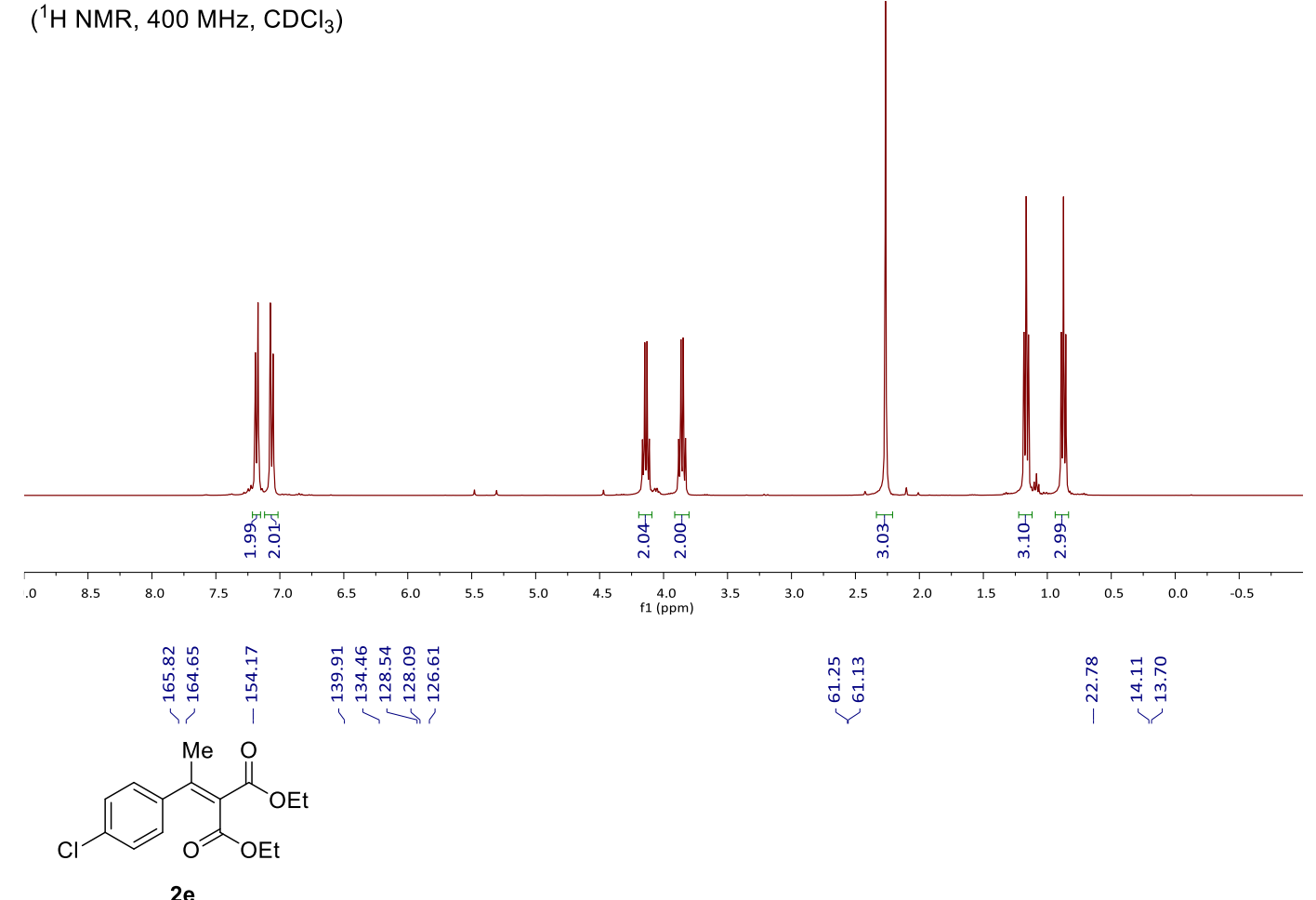

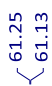

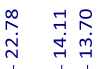

$\left({ }^{13} \mathrm{C} \mathrm{NMR}, 101 \mathrm{MHz}, \mathrm{CDCl}_{3}\right)$

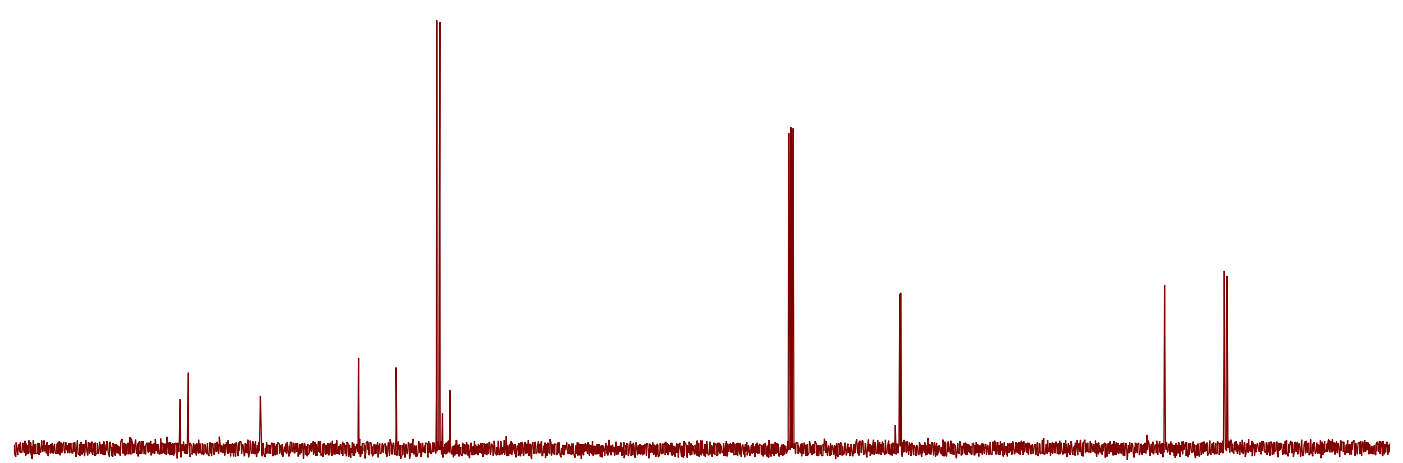

\begin{tabular}{llllllllllllllllllllllll}
\hline 90 & 180 & 170 & 160 & 150 & 140 & 130 & 120 & 110 & 100 & 90 & 80 & 70 & 60 & 50 & 40 & 30 & 20 & 10 & 0 & $-\vdots$
\end{tabular} 
Diethyl-2-(1-(4-bromophenyl)ethylidene)malonate (2f)

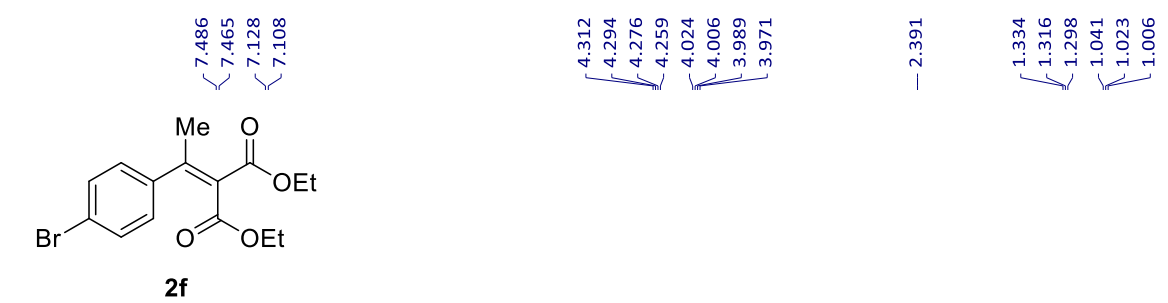

( ${ }^{1} \mathrm{H}$ NMR, $\left.400 \mathrm{MHz}, \mathrm{CDCl}_{3}\right)$
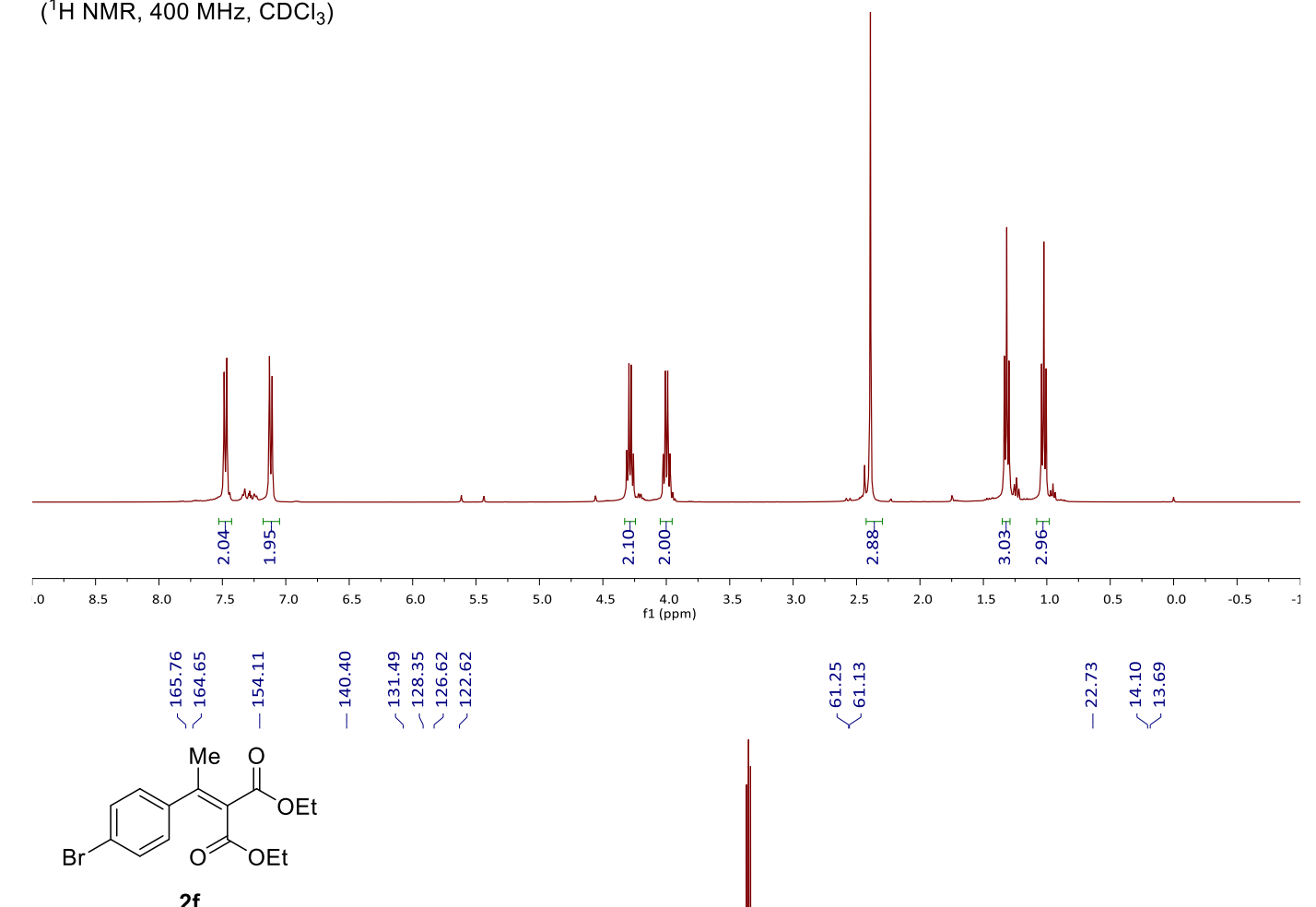

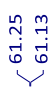

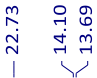

$\left({ }^{13} \mathrm{C} \mathrm{NMR}, 101 \mathrm{MHz}, \mathrm{CDCl}_{3}\right)$

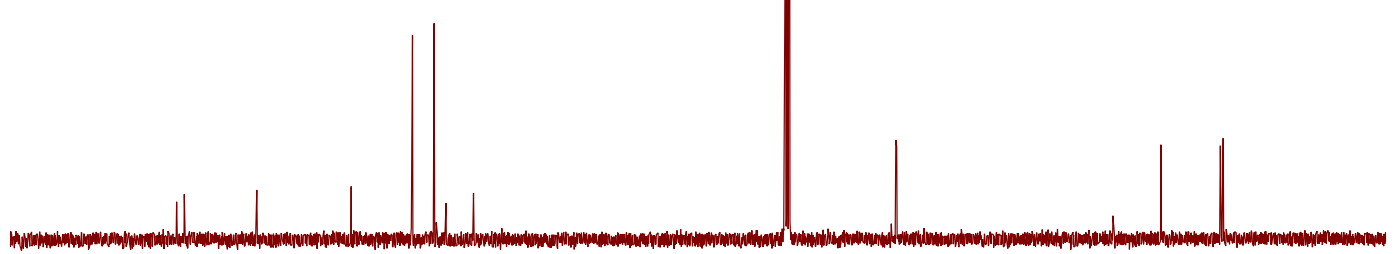

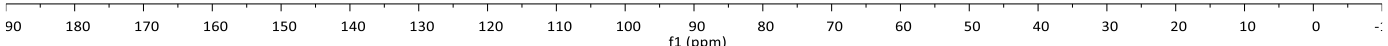


Diethyl-2-(1-(4-(trifluoromethyl)phenyl)ethylidene)malonate (2g)
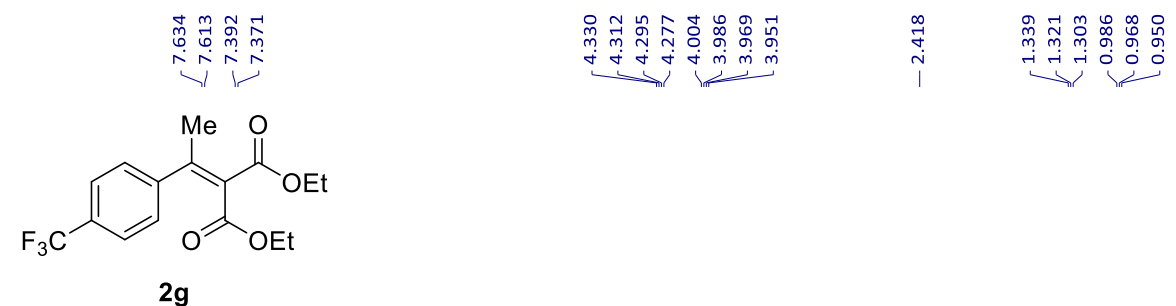

$\left({ }^{1} \mathrm{H} \mathrm{NMR}, 400 \mathrm{MHz}, \mathrm{CDCl}_{3}\right)$
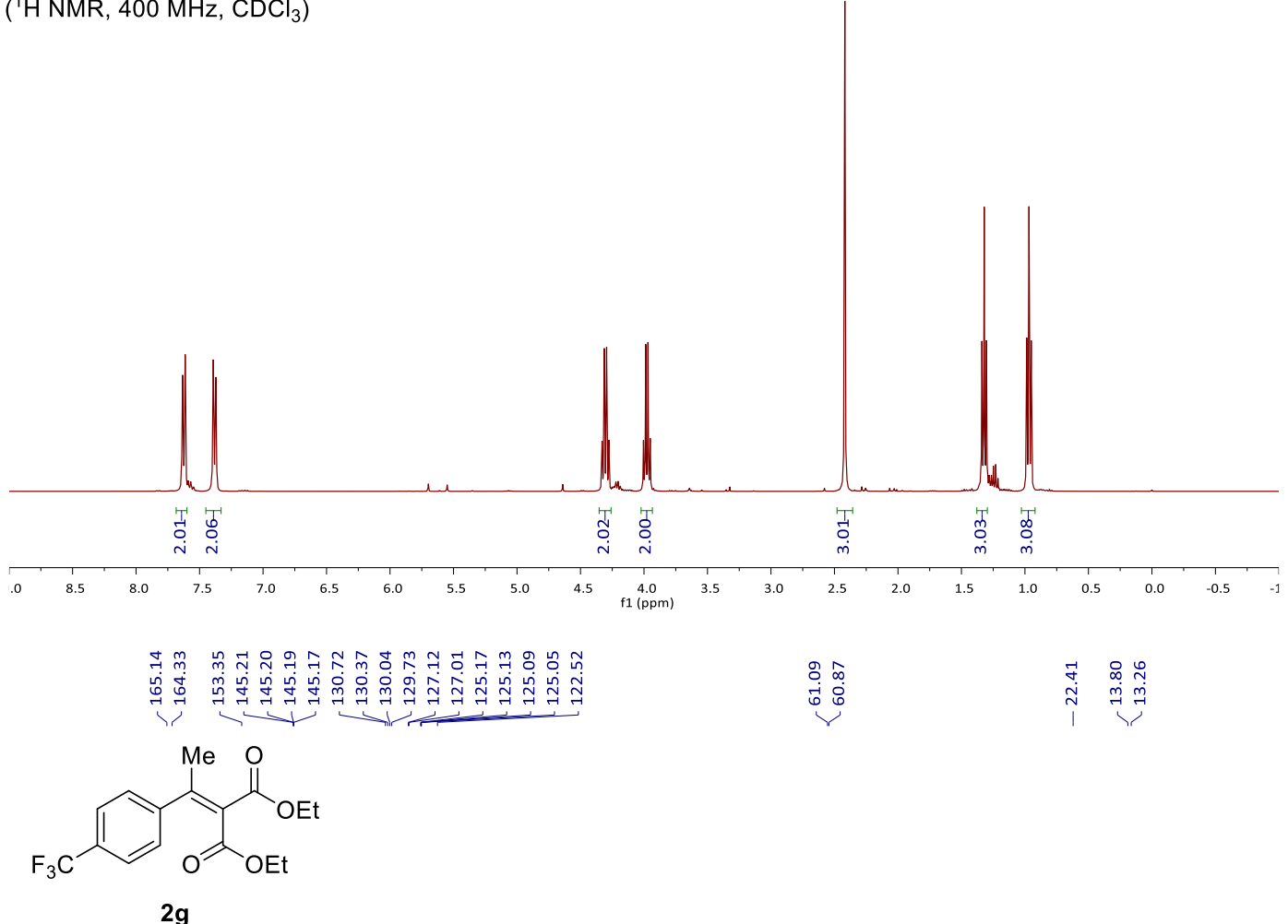

$\left({ }^{13} \mathrm{C} \mathrm{NMR}, 101 \mathrm{MHz}, \mathrm{CDCl}_{3}\right)$

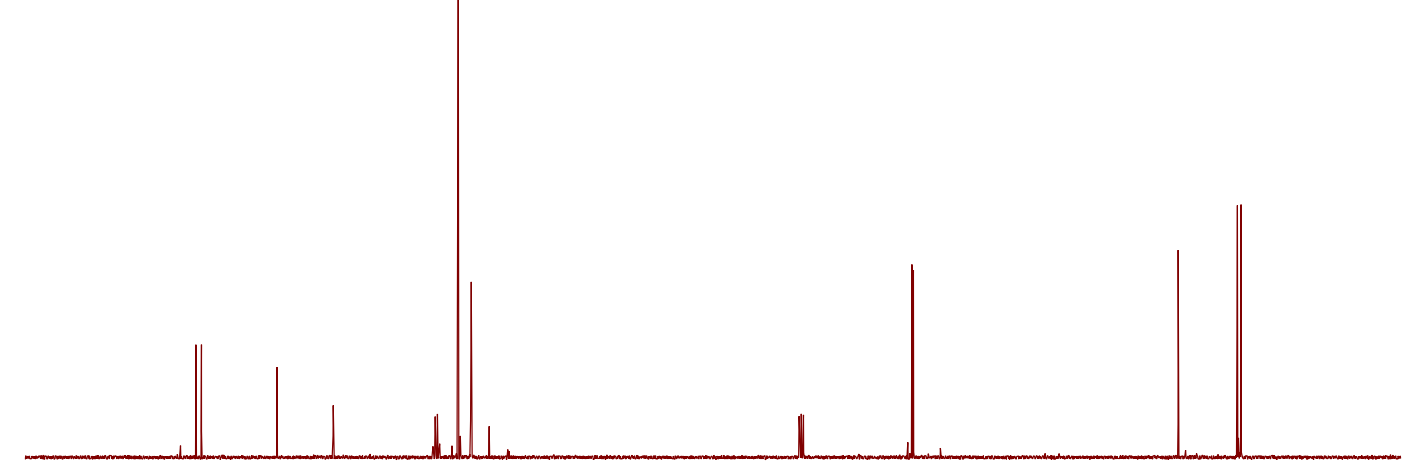

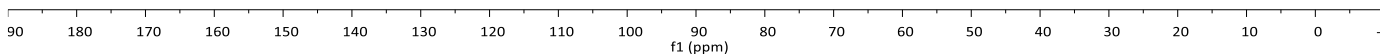


Diethyl-2-(1-(4-(ethoxycarbonyl)phenyl)ethylidene)malonate (2h)
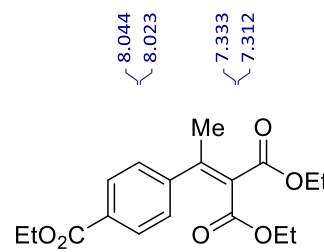

$2 \mathrm{~h}$

$\left({ }^{1} \mathrm{H}\right.$ NMR, $\left.400 \mathrm{MHz}, \mathrm{CDCl}_{3}\right)$
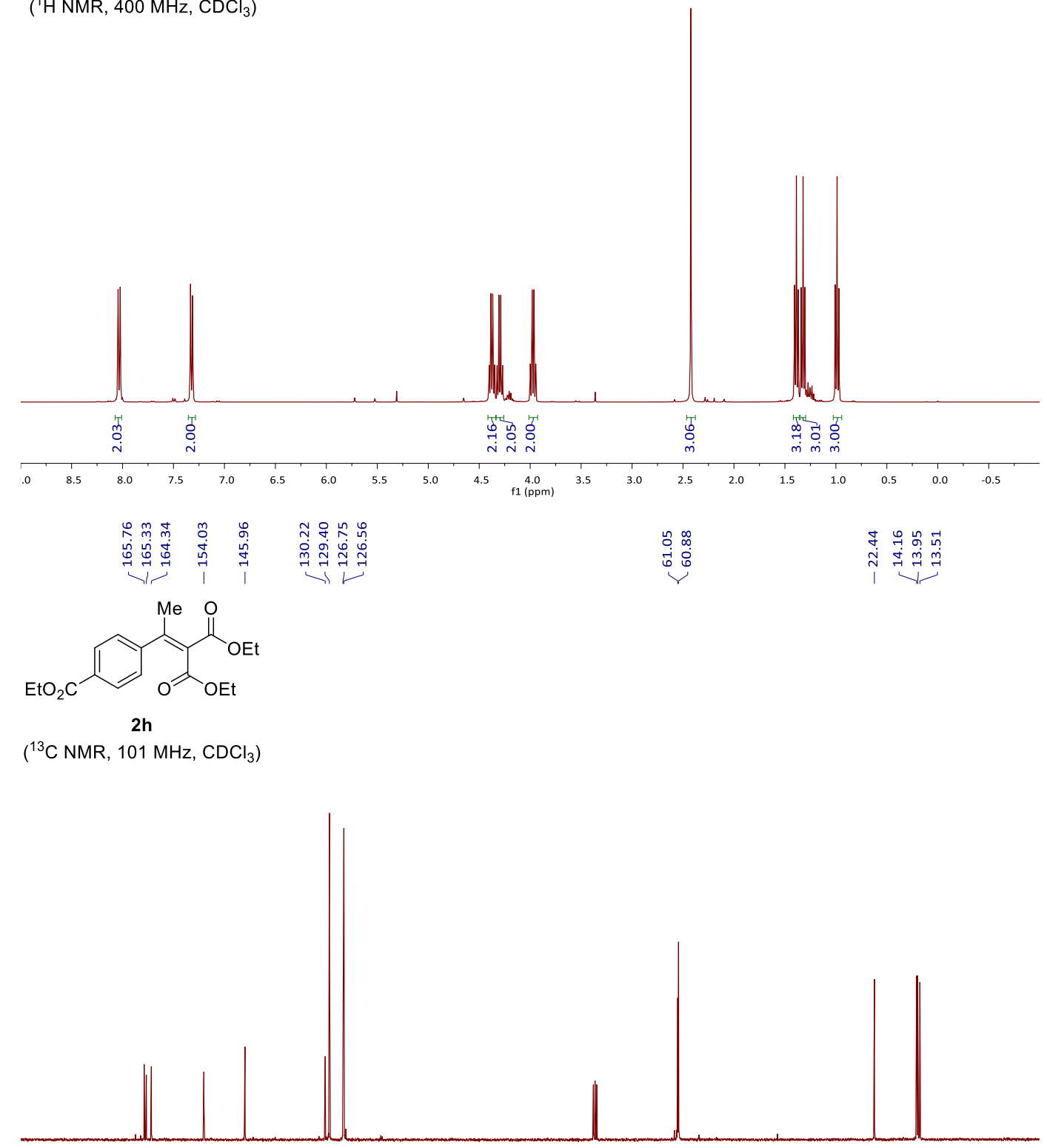

\begin{tabular}{lllllllllllllllllllllllll}
\hline 90 & 180 & 170 & 160 & 150 & 140 & 130 & 120 & 110 & 100 & 90 & 80 & 70 & 60 & 50 & 40 & 30 & 20 & 10 & 0 & $-:$
\end{tabular} 
Diethyl-2-(1-([1,1'-biphenyl]-4-yl)ethylidene)malonate (2i)

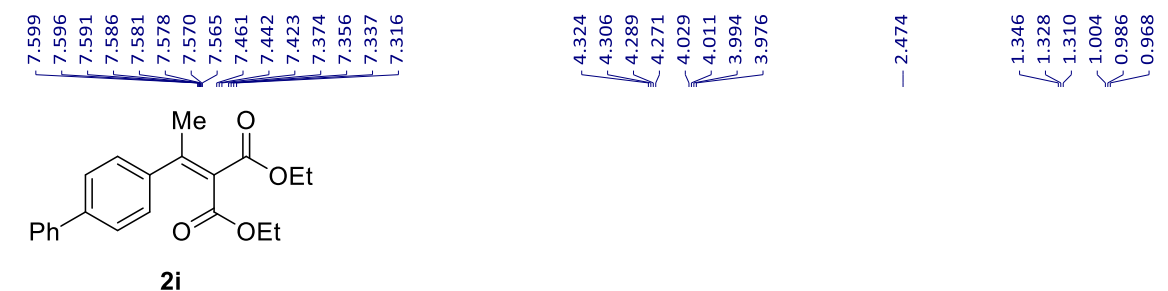

$\left({ }^{1} \mathrm{H} \mathrm{NMR}, 400 \mathrm{MHz}, \mathrm{CDCl}_{3}\right)$
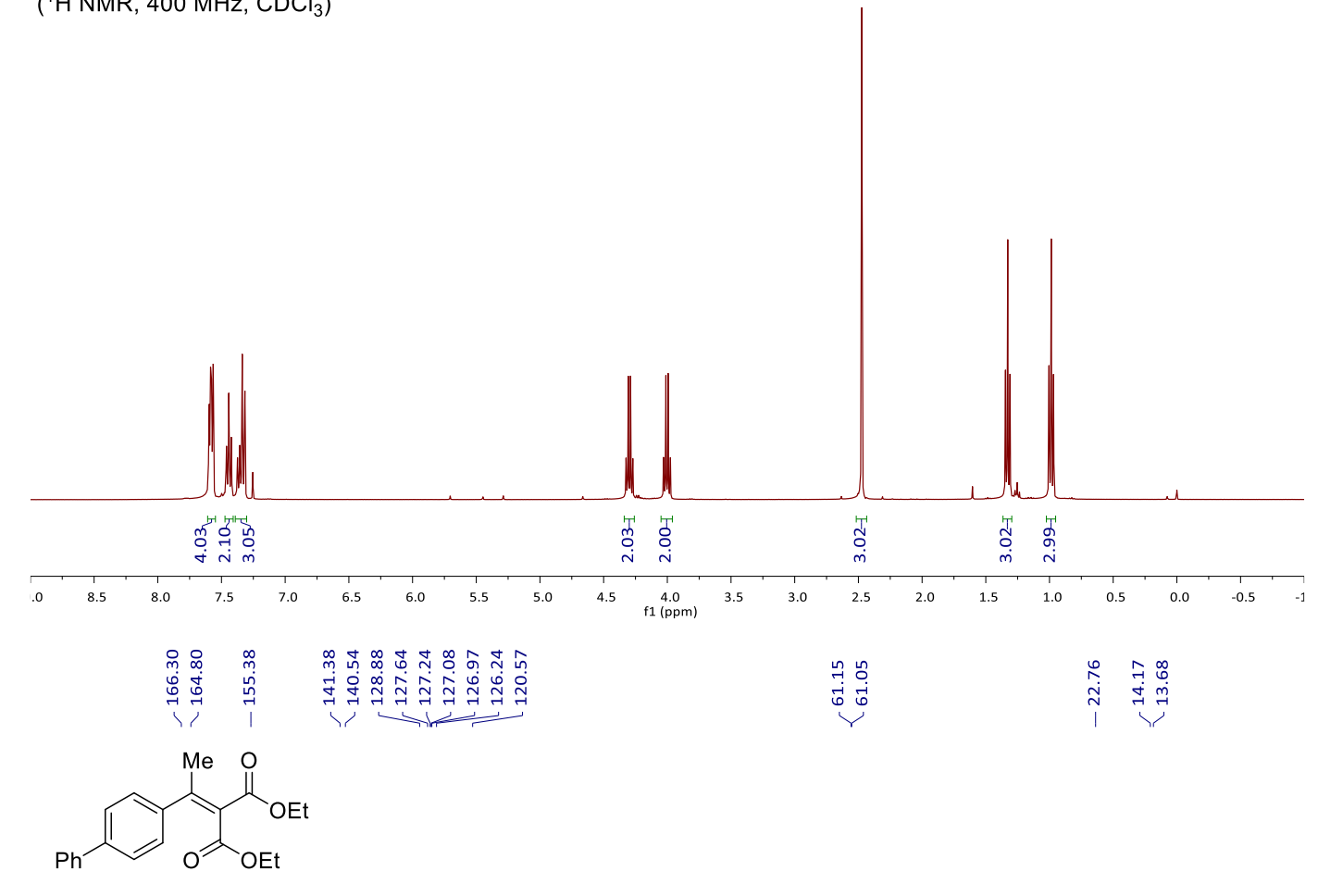

$\left({ }^{13} \mathrm{C} \mathrm{NMR}, 101 \mathrm{MHz}, \mathrm{CDCl}_{3}\right)$

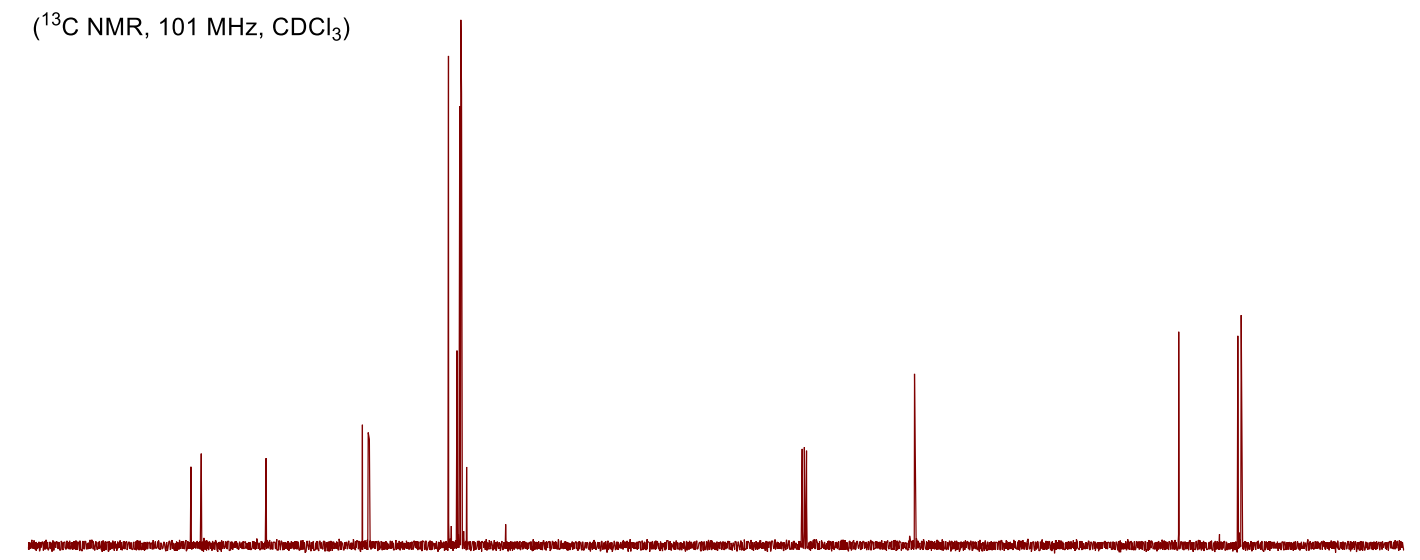

\begin{tabular}{llllllllllllllllllll}
\hline 90 & 180 & 170 & 160 & 150 & 140 & 130 & 120 & 110 & 100 & $\begin{array}{l}100 \\
f 1(\mathrm{pm})\end{array}$ & 80 & 70 & 60 & 50 & 40 & 30 & 20 & 10 & 0
\end{tabular} 
Diethyl 2-(1-(p-tolyl)ethylidene)malonate $(2 \mathrm{j})$

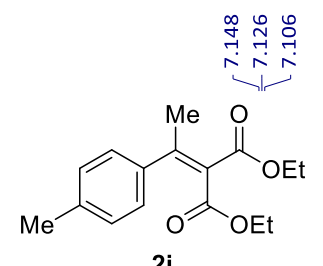

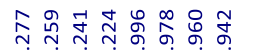

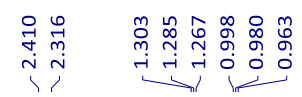

$\left({ }^{1} \mathrm{H} \mathrm{NMR}, 400 \mathrm{MHz}, \mathrm{CDCl}_{3}\right)$

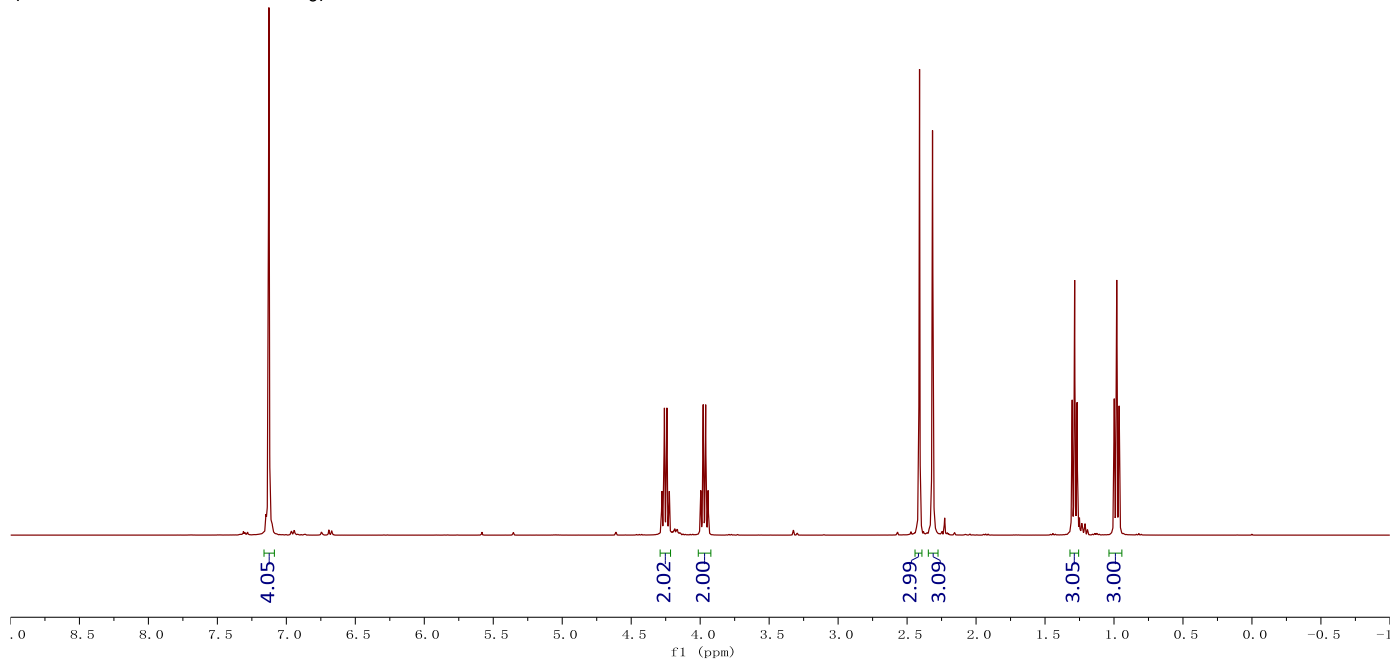

Diethyl 2-(1-(4-methoxyphenyl)ethylidene)malonate (2k)
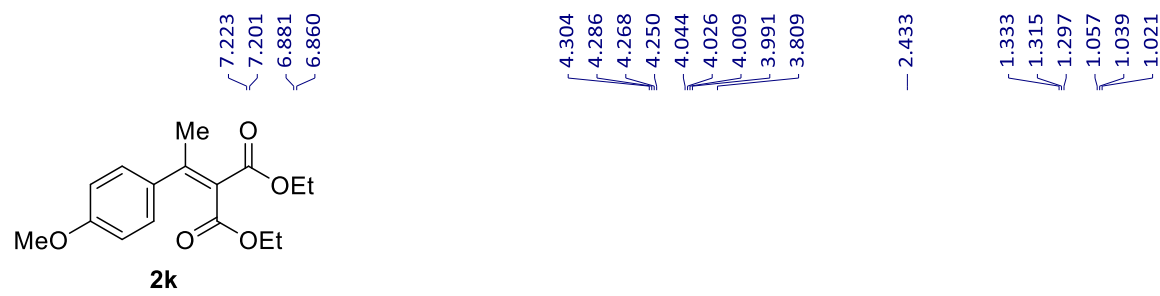

$\left({ }^{1} \mathrm{H} \mathrm{NMR}, 400 \mathrm{MHz}, \mathrm{CDCl}_{3}\right)$

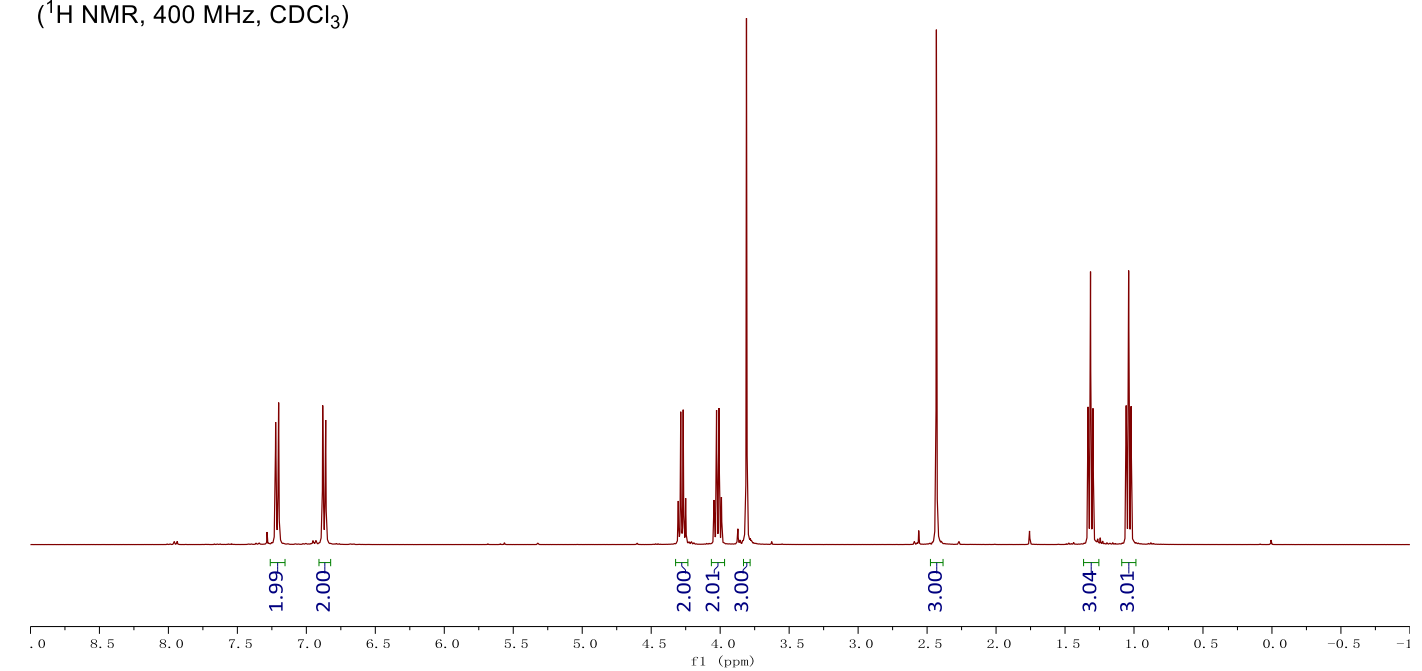


Diethyl-2-(1-(3-fluorophenyl)ethylidene)malonate (2I)
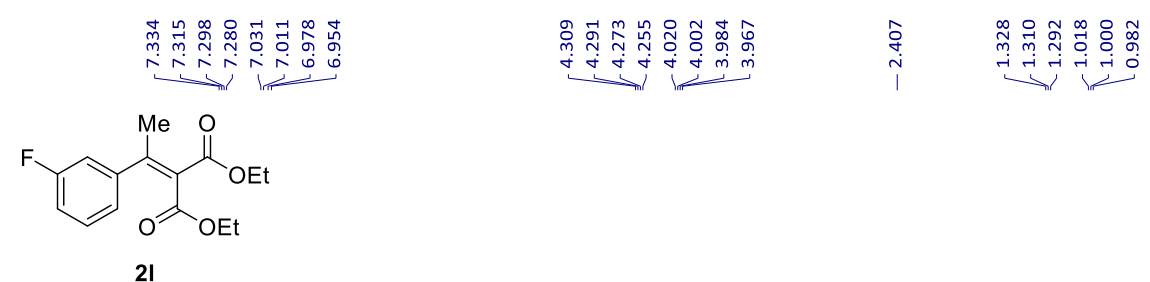

$\left({ }^{1} \mathrm{H}\right.$ NMR, $\left.400 \mathrm{MHz}, \mathrm{CDCl}_{3}\right)$

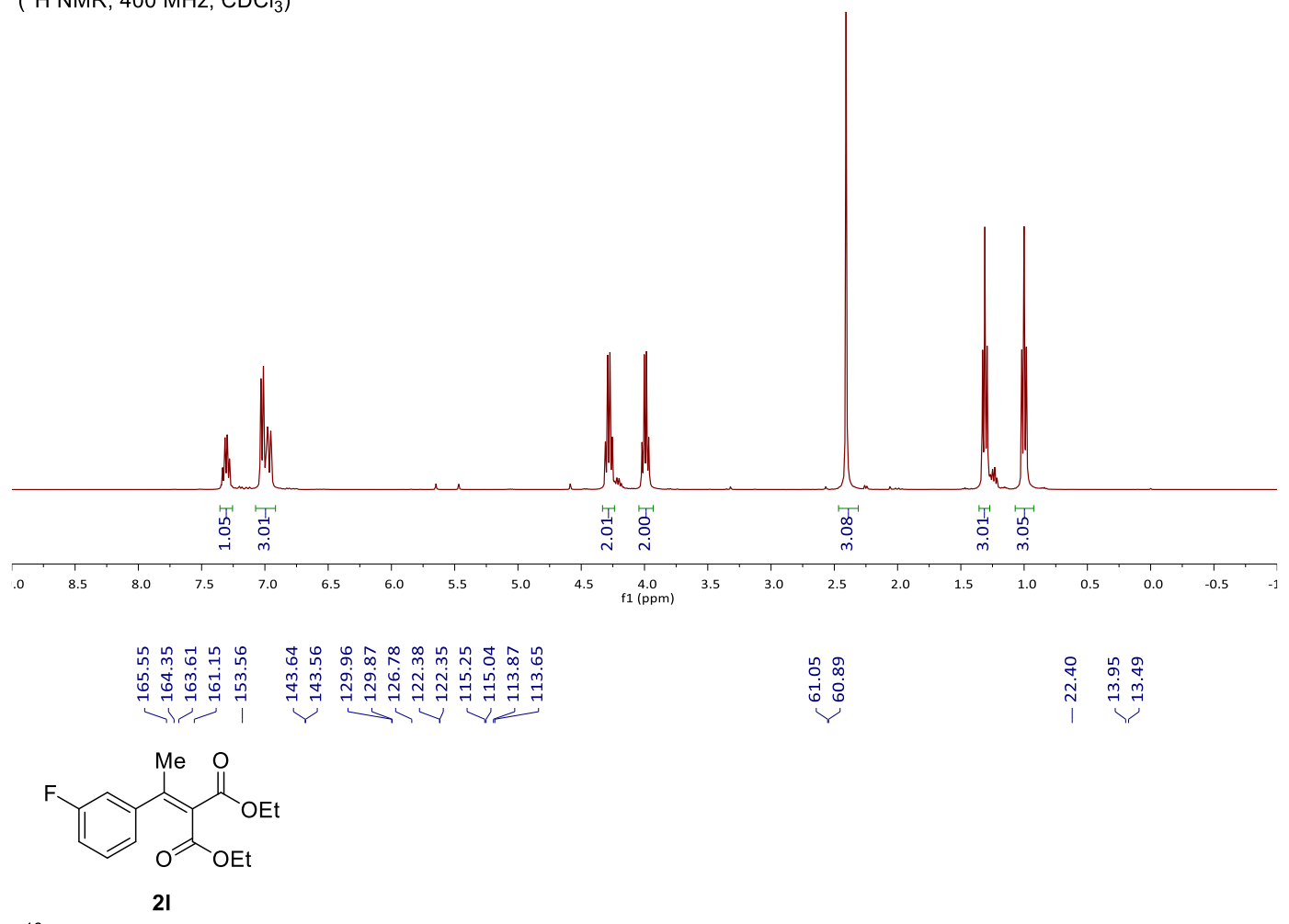

$\left({ }^{13} \mathrm{C} \mathrm{NMR}, 101 \mathrm{MHz}, \mathrm{CDCl}_{3}\right)$

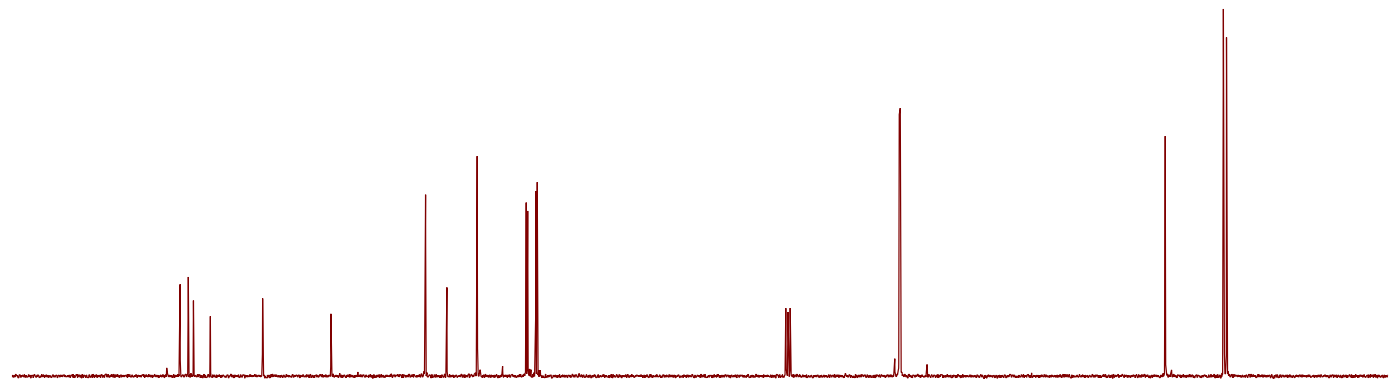

\begin{tabular}{lllllllllllllllllllllll}
\hline & 180 & 170 & 160 & 150 & 140 & 130 & 120 & 110 & 100 & 90 & 80 & 70 & 60 & 50 & 40 & 30 & 20 & 10 & 0 & $-:$
\end{tabular} 
Diethyl-2-(1-(m-tolyl)ethylidene)malonate (2m)

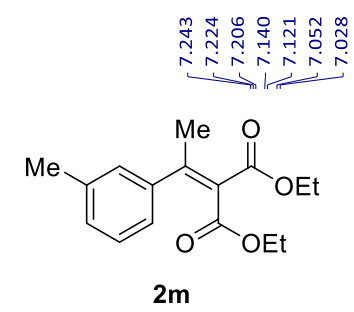

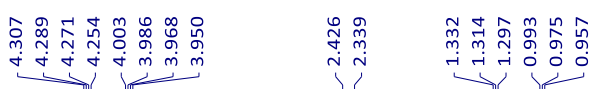

( ${ }^{1} \mathrm{H} \mathrm{NMR}, 400 \mathrm{MHz}, \mathrm{CDCl}_{3}$ )

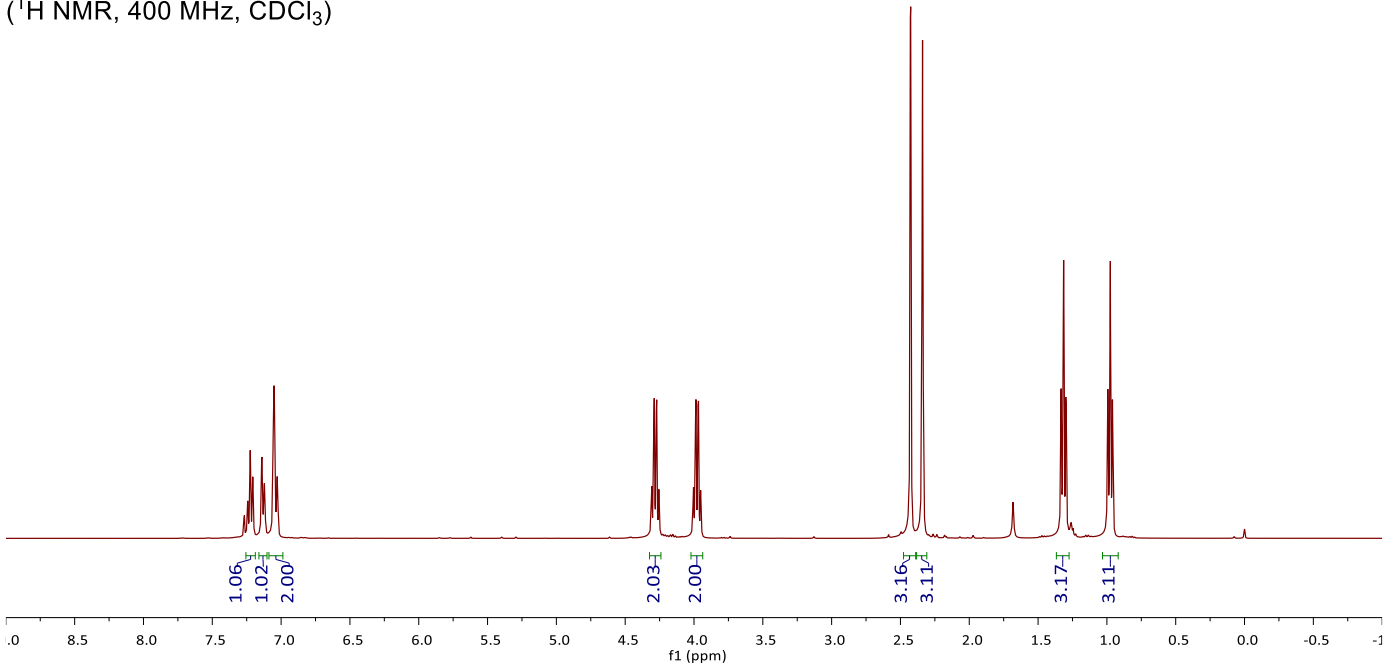

일 \%

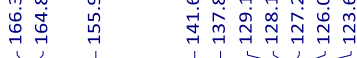

望

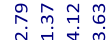

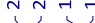

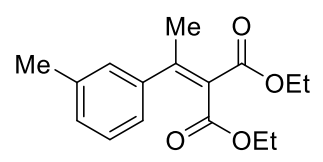

$2 \mathrm{~m}$

$\left({ }^{13} \mathrm{C} \mathrm{NMR}, 101 \mathrm{MHz}, \mathrm{CDCl}_{3}\right)$

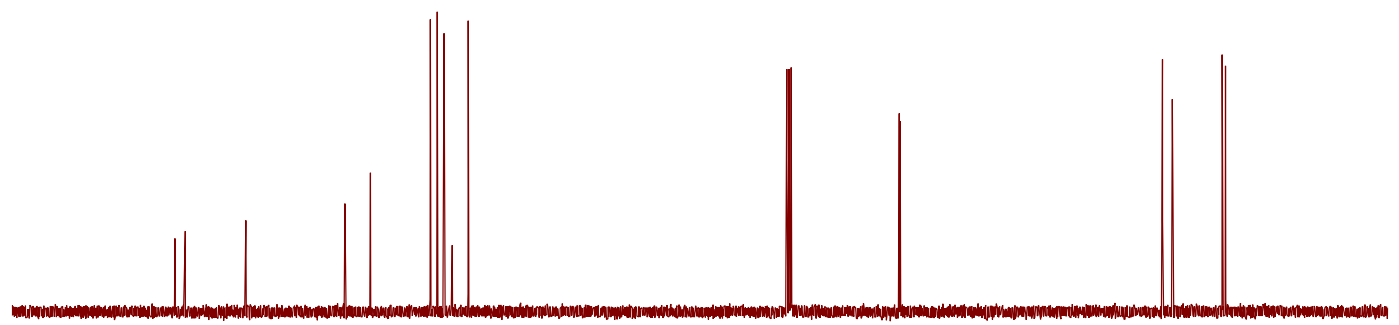

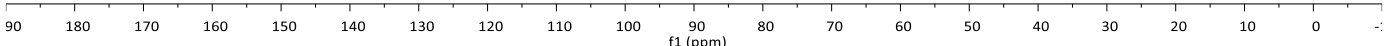


Diethyl-2-(1-(3-methoxyphenyl)ethylidene)malonate (2n)

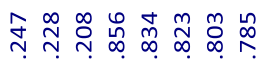

r

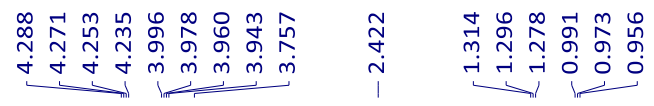

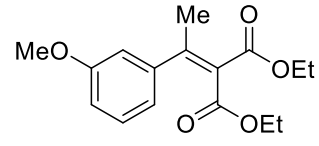

2n

$\left({ }^{1} \mathrm{H} \mathrm{NMR}, 400 \mathrm{MHz}, \mathrm{CDCl}_{3}\right)$

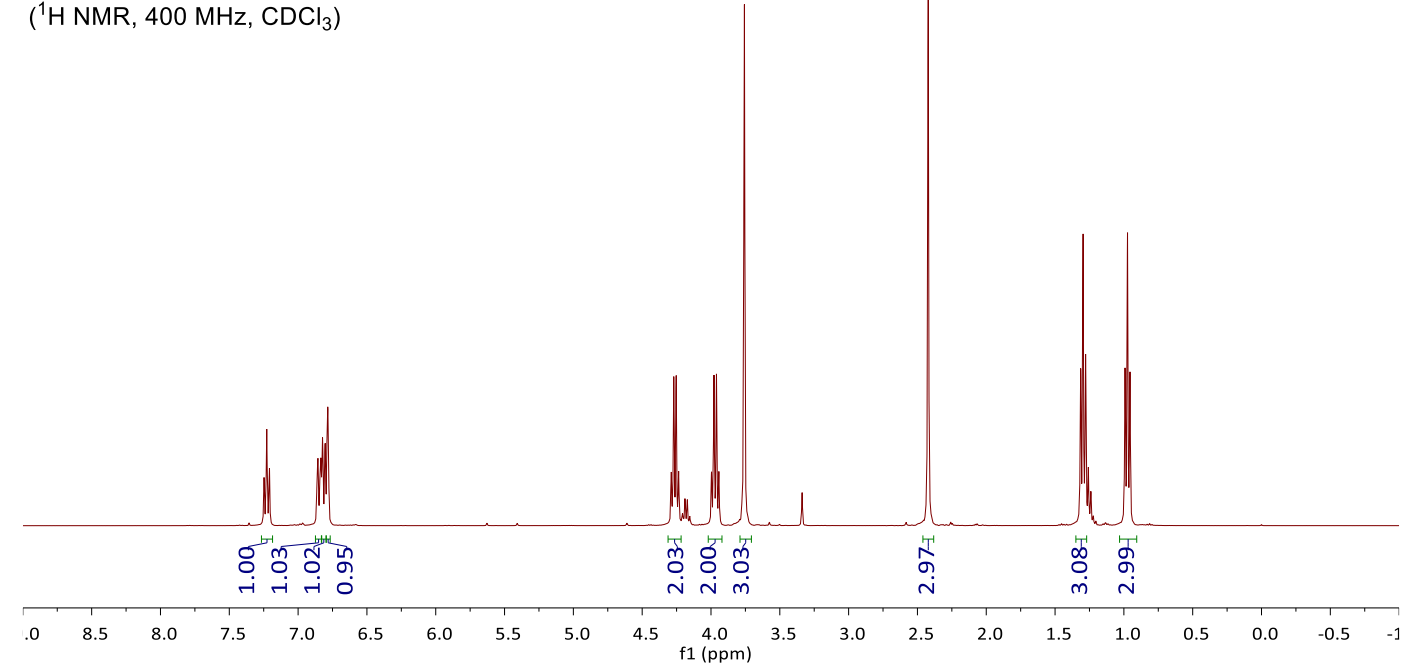

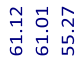

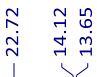

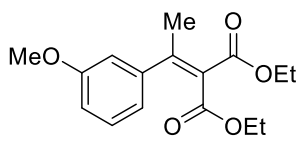

2n

$\left({ }^{13} \mathrm{C} \mathrm{NMR}, 101 \mathrm{MHz}, \mathrm{CDCl}_{3}\right)$

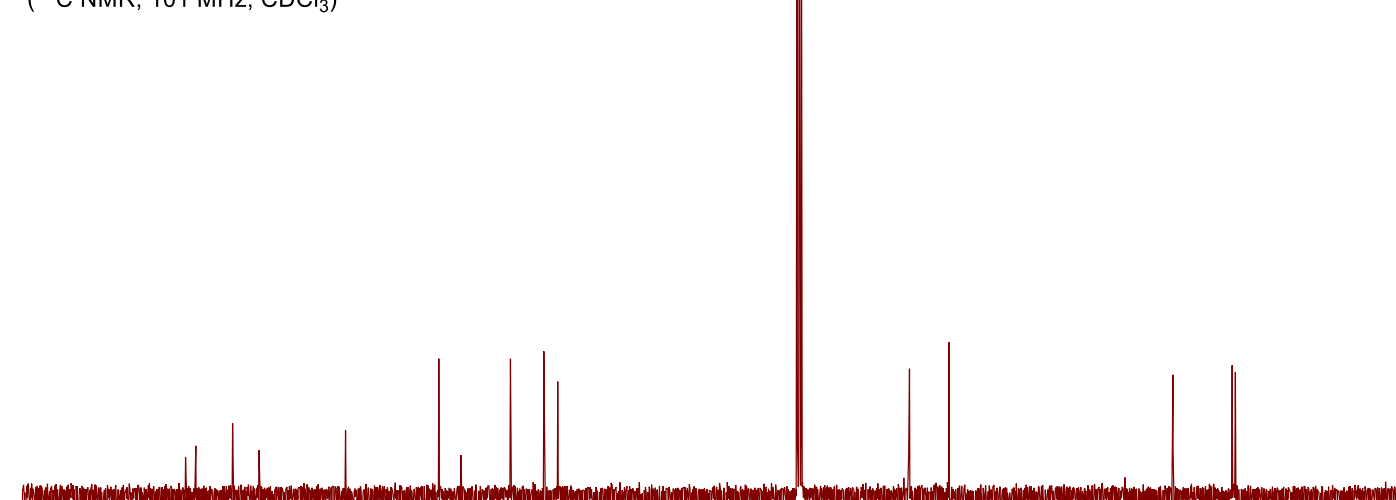

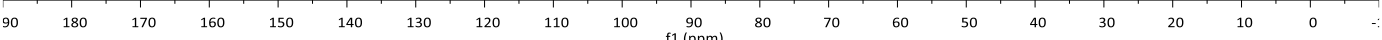


Diethyl-2-(1-(2-fluorophenyl)ethylidene)malonate (2o)

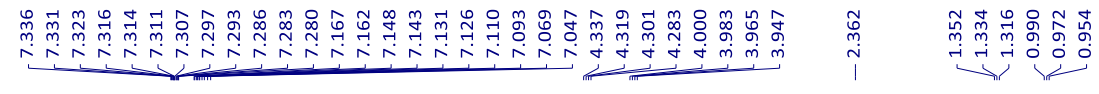

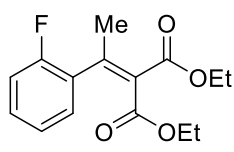

20

$\left({ }^{1} \mathrm{H} \mathrm{NMR}, 400 \mathrm{MHz}, \mathrm{CDCl}_{3}\right)$

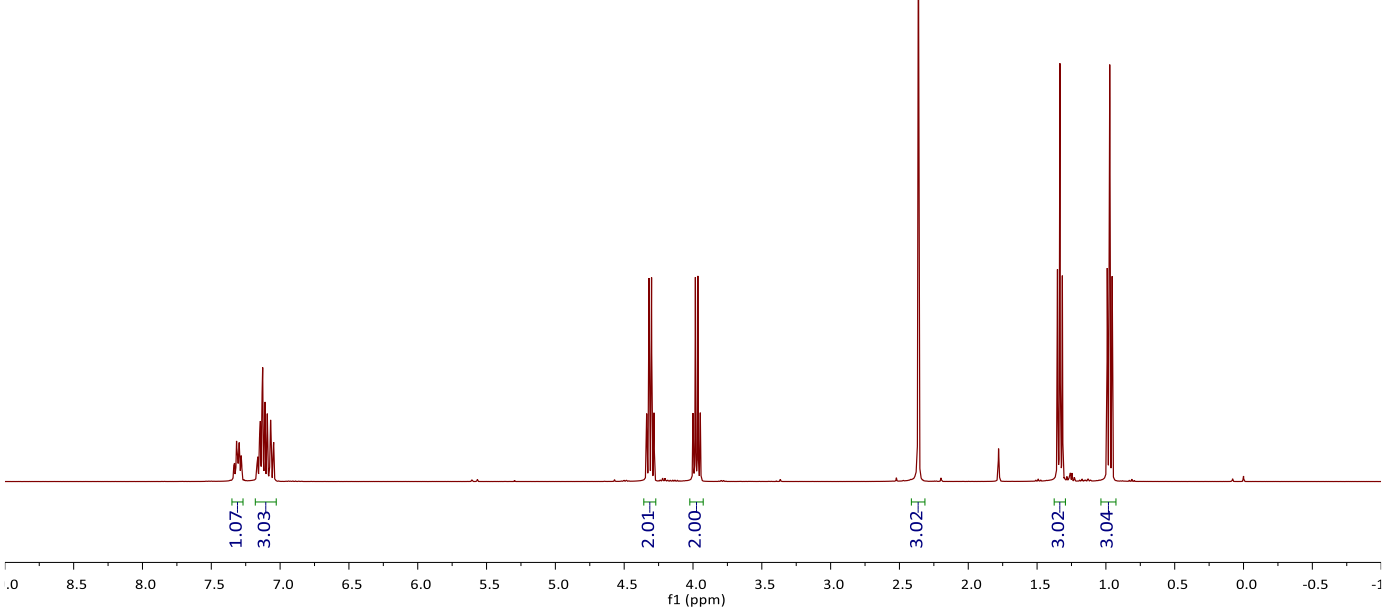

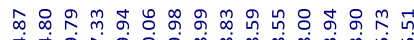

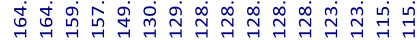

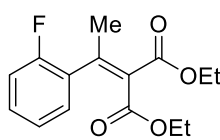

20

$\left({ }^{13} \mathrm{C} \mathrm{NMR}, 101 \mathrm{MHz}, \mathrm{CDCl}_{3}\right)$

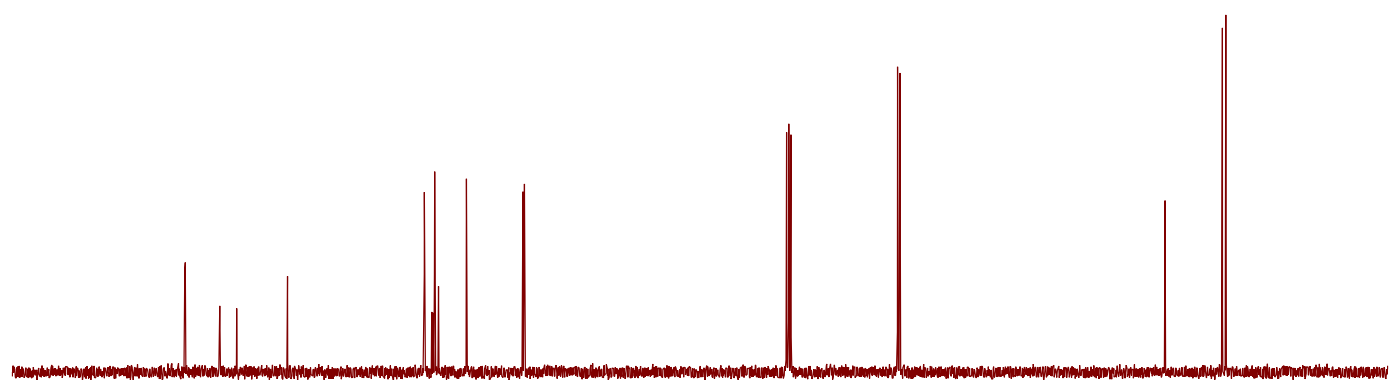

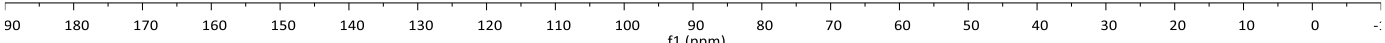


Diethyl-2-(1-(o-tolyl)ethylidene)malonate (2p)

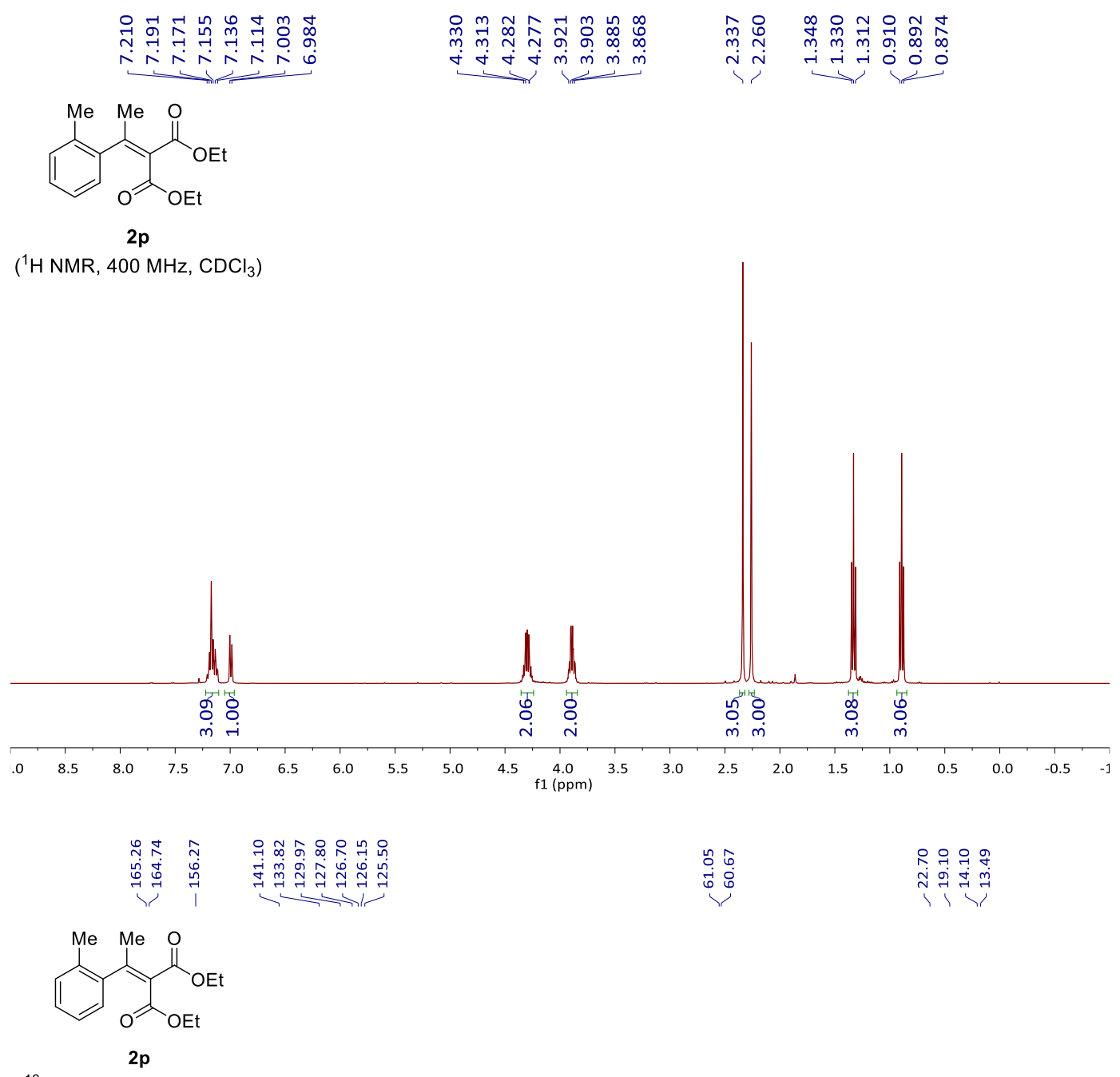

$\left({ }^{13} \mathrm{C} \mathrm{NMR,}, 101 \mathrm{MHz}, \mathrm{CDCl}_{3}\right)$

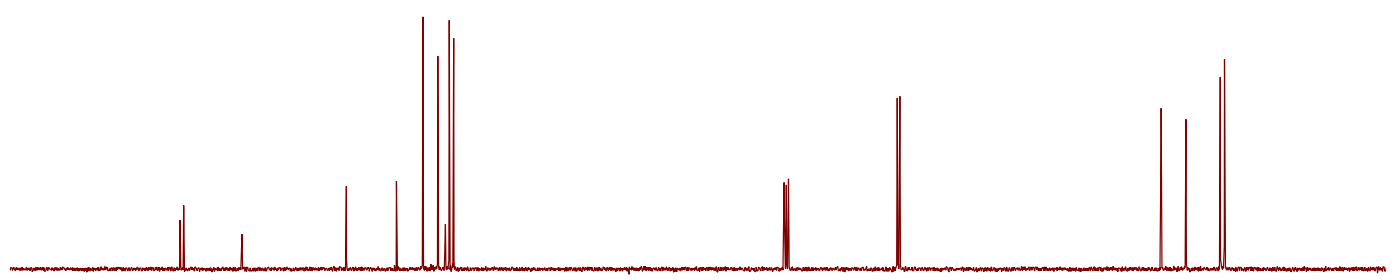

$\begin{array}{llllllllllllllllllllllllllll}90 & 180 & 170 & 160 & 150 & 140 & 130 & 120 & 110 & 100 & \begin{array}{c}90 \\ \mathrm{f} 1(\mathrm{ppm})\end{array} & 80 & 70 & 60 & 50 & 40 & 30 & 20 & 10 & 0 & \vdots\end{array}$ 
Diethyl-2-(1-(benzo[d][1,3]dioxol-5-yl)ethylidene)malonate (2q)

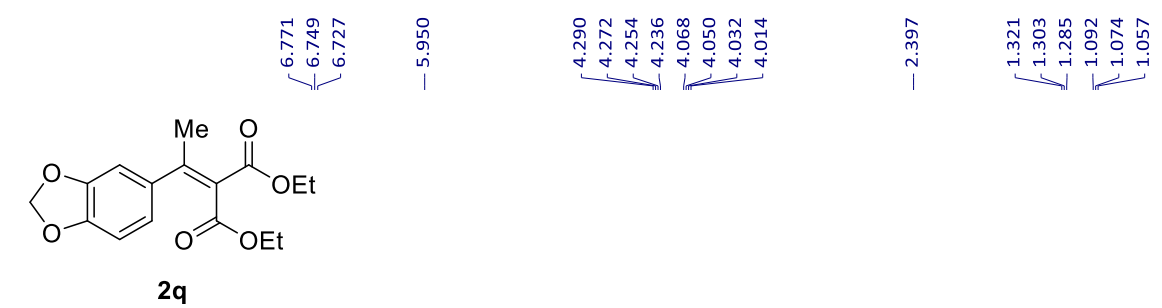

$\left({ }^{1} \mathrm{H}\right.$ NMR, $\left.400 \mathrm{MHz}, \mathrm{CDCl}_{3}\right)$
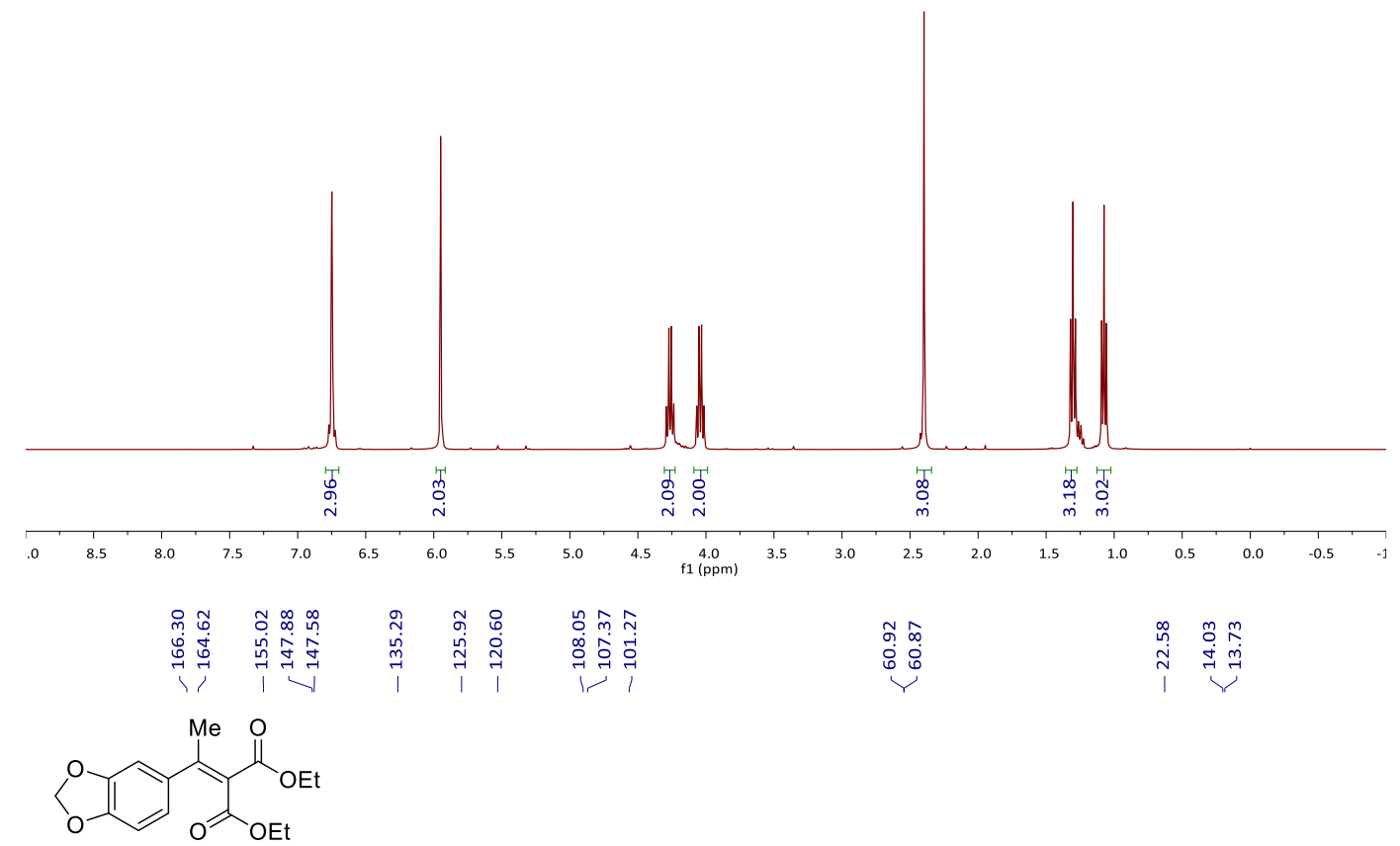

$2 q$

$\left({ }^{13} \mathrm{C} \mathrm{NMR}, 101 \mathrm{MHz}, \mathrm{CDCl}_{3}\right)$

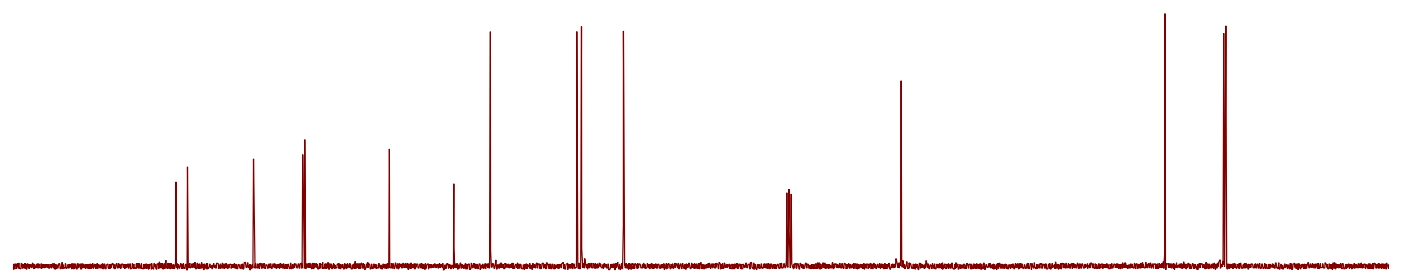

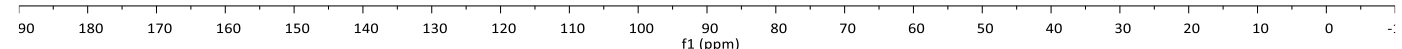


Diethyl-2-(1-(naphthalen-1-yl)ethylidene)malonate (2r)

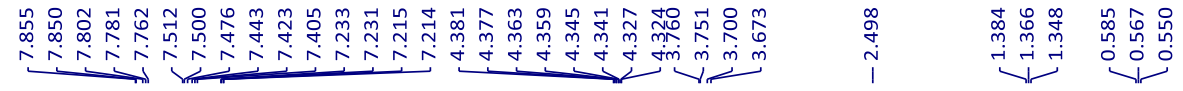

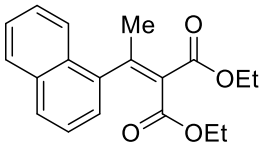

$2 \mathbf{r}$

$\left({ }^{1} \mathrm{H} \mathrm{NMR}, 400 \mathrm{MHz}, \mathrm{CDCl}_{3}\right)$

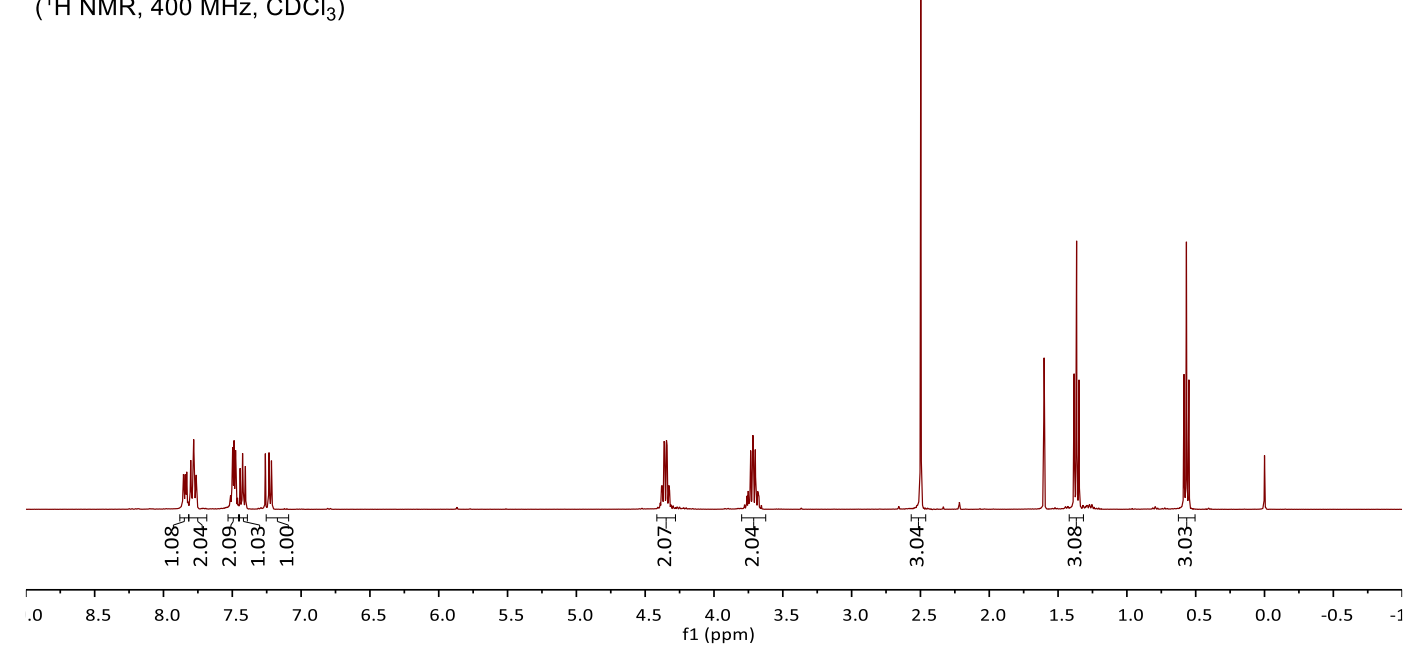

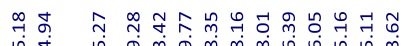

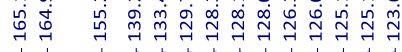

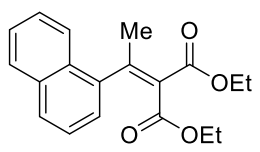

$2 \mathbf{r}$

$\left({ }^{13} \mathrm{C} \mathrm{NMR}, 101 \mathrm{MHz}, \mathrm{CDCl}_{3}\right)$

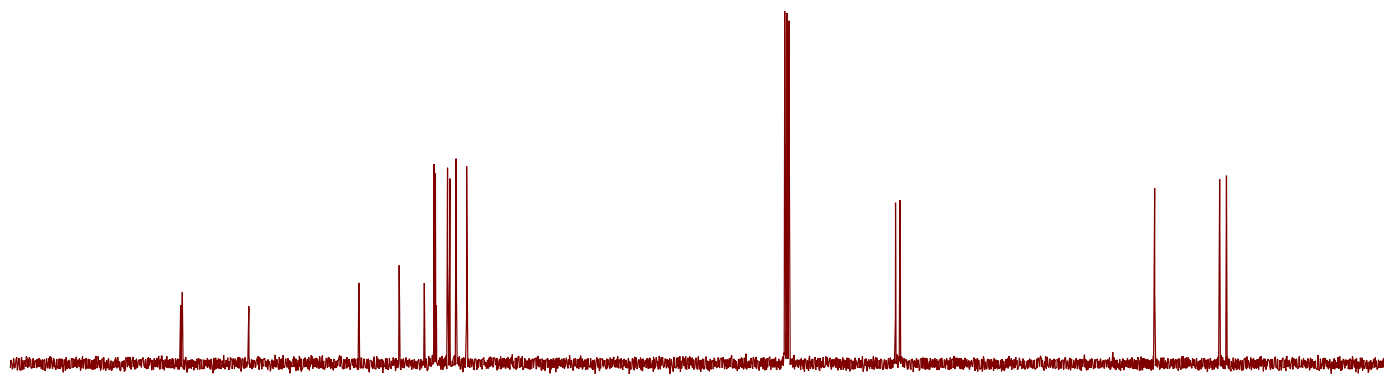

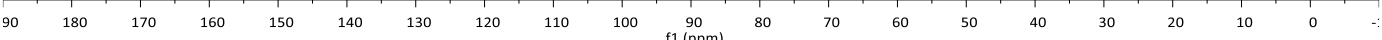


Diethyl-2-(1-(naphthalen-2-yl)ethylidene)malonate (2s)

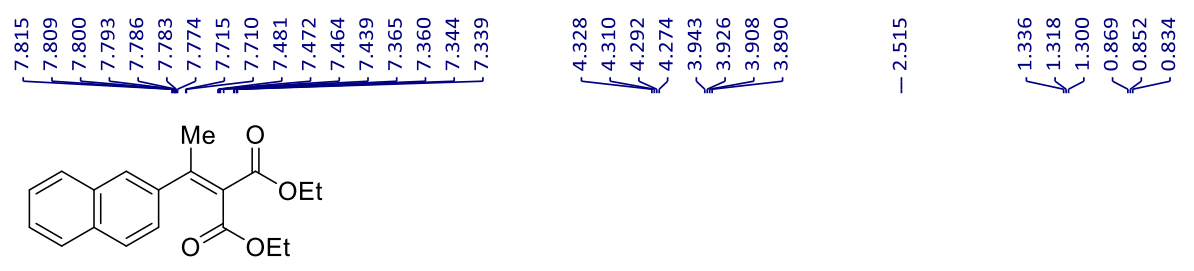

2s

( $\left.{ }^{1} \mathrm{H} \mathrm{NMR}, 400 \mathrm{MHz}, \mathrm{CDCl}_{3}\right)$
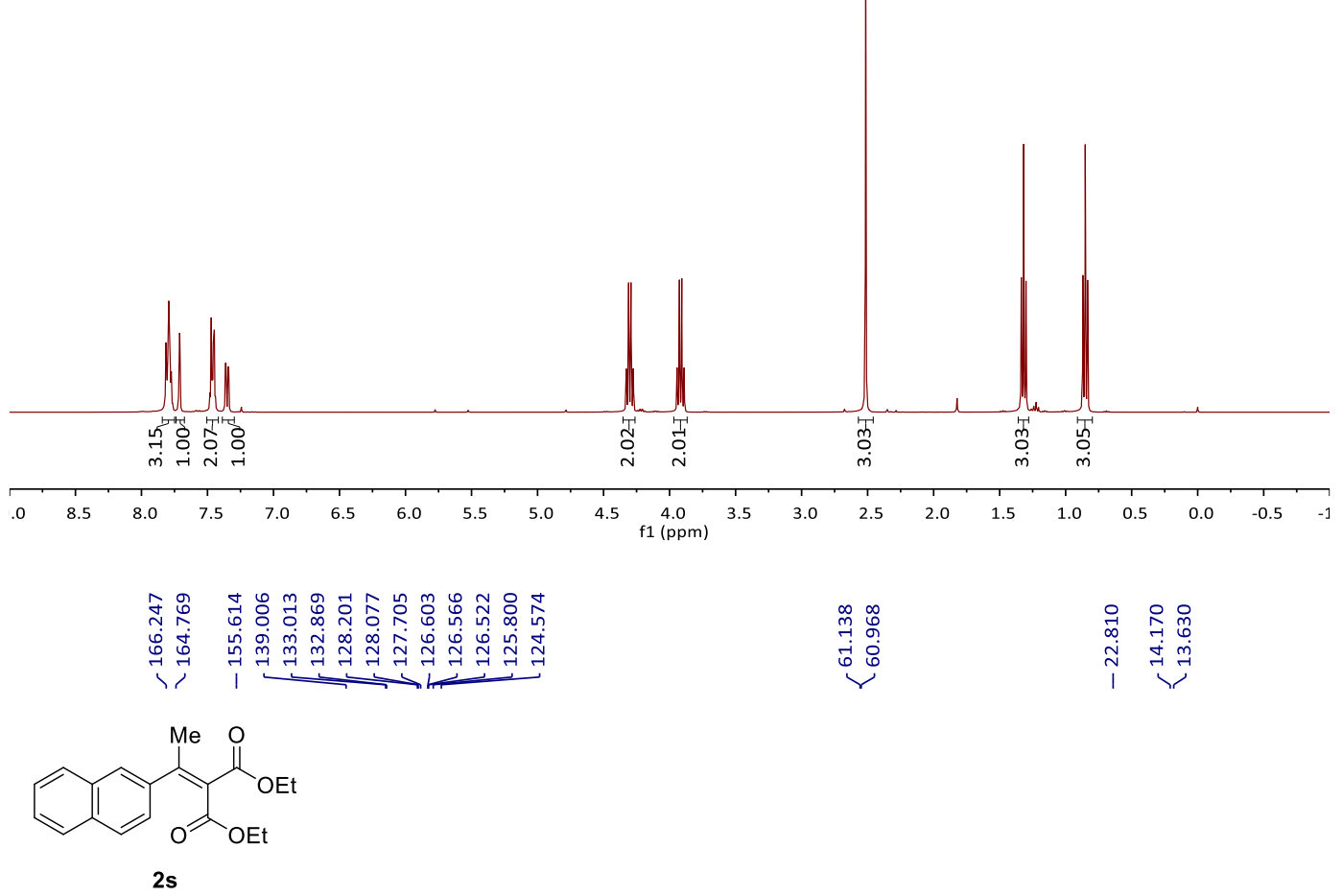

$\left({ }^{13} \mathrm{C} \mathrm{NMR}, 101 \mathrm{MHz}, \mathrm{CDCl}_{3}\right)$

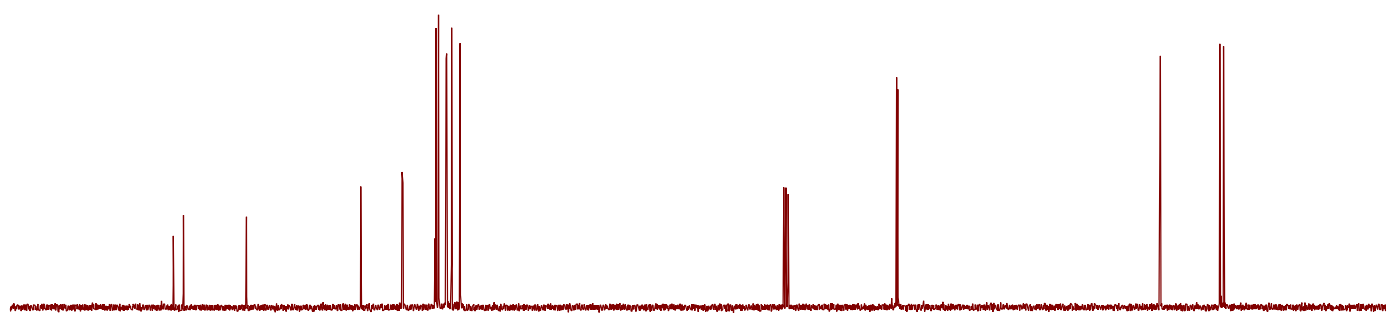

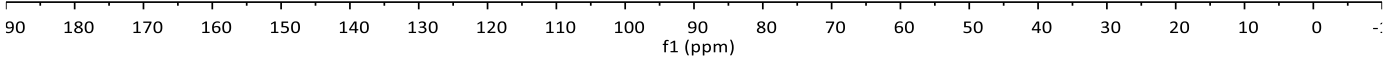


Diethyl-2-(1-(thiophen-2-yl)ethylidene)malonate (2t) (with a minor double bond migrated isomer)
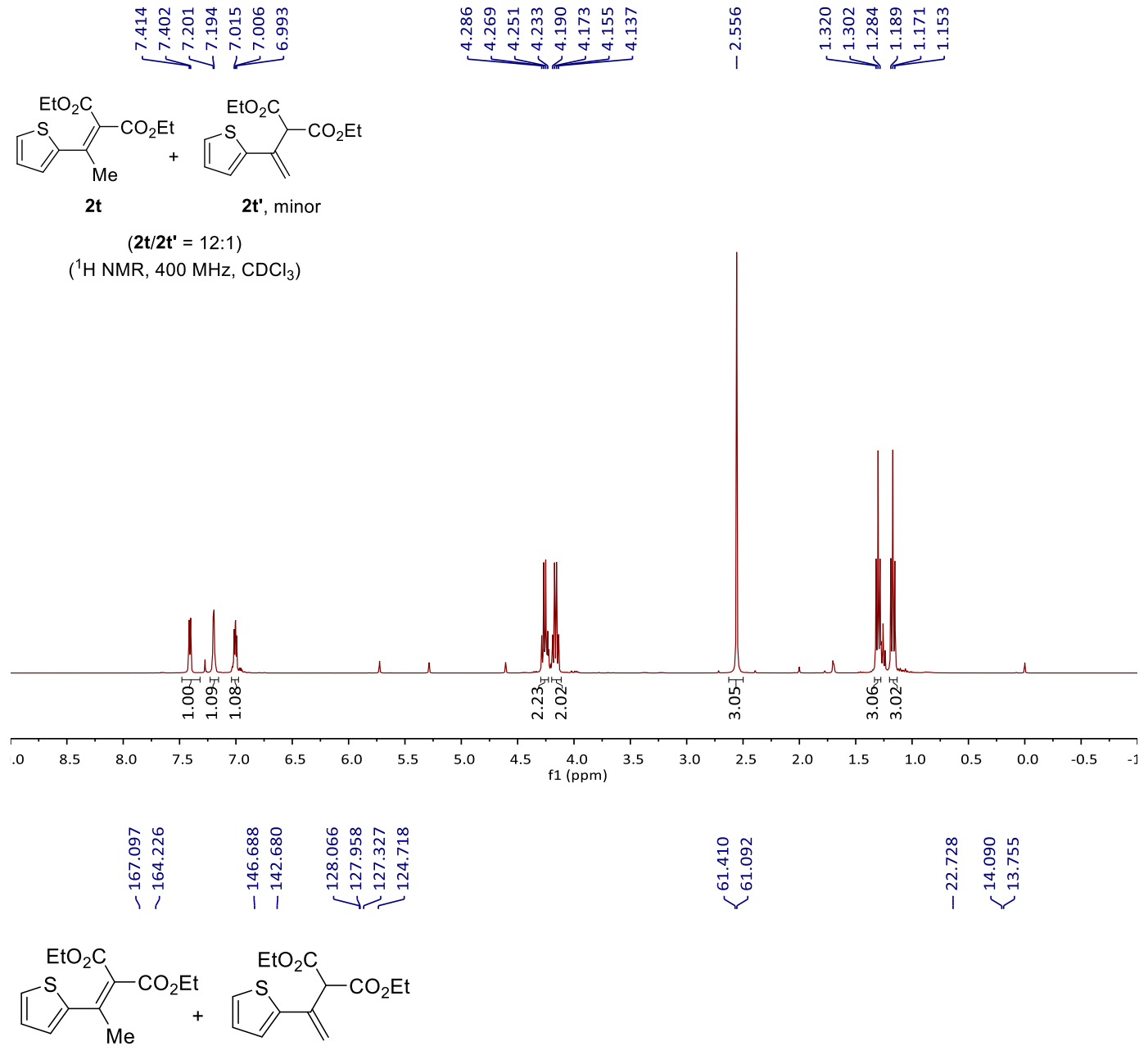

$2 t$

2t', minor

$\left(\mathbf{2 t} / \mathbf{2} \mathbf{t}^{\prime}=12: 1\right)$

$\left({ }^{13} \mathrm{C} \mathrm{NMR}, 101 \mathrm{MHz}, \mathrm{CDCl}_{3}\right)$

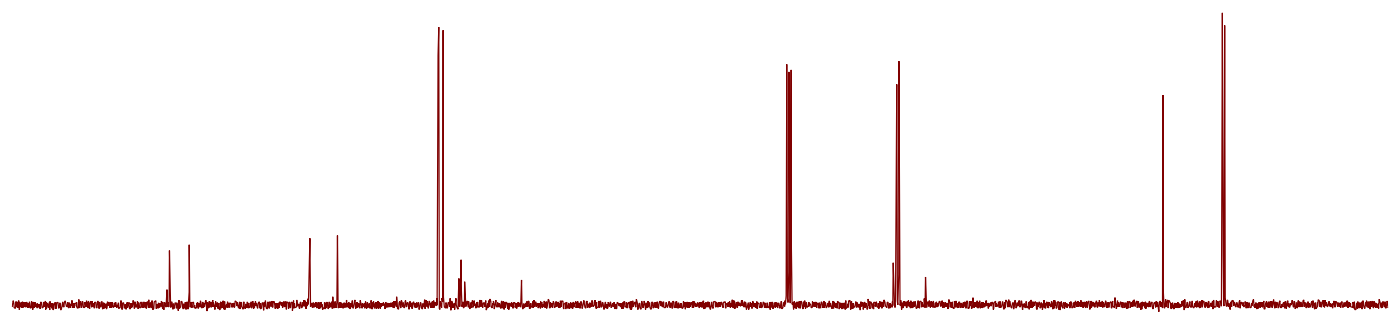

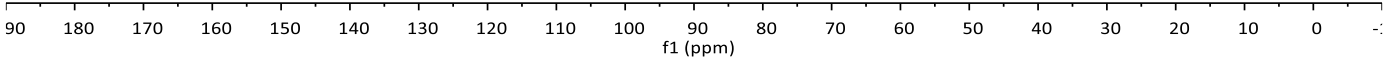


Diethyl-2-(1-(furan-2-yl)ethylidene)malonate (2u)

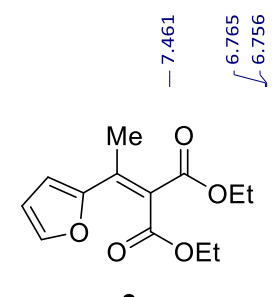

$2 \mathbf{u}$

$\left({ }^{1} \mathrm{H}\right.$ NMR, $\left.400 \mathrm{MHz}, \mathrm{CDCl}_{3}\right)$

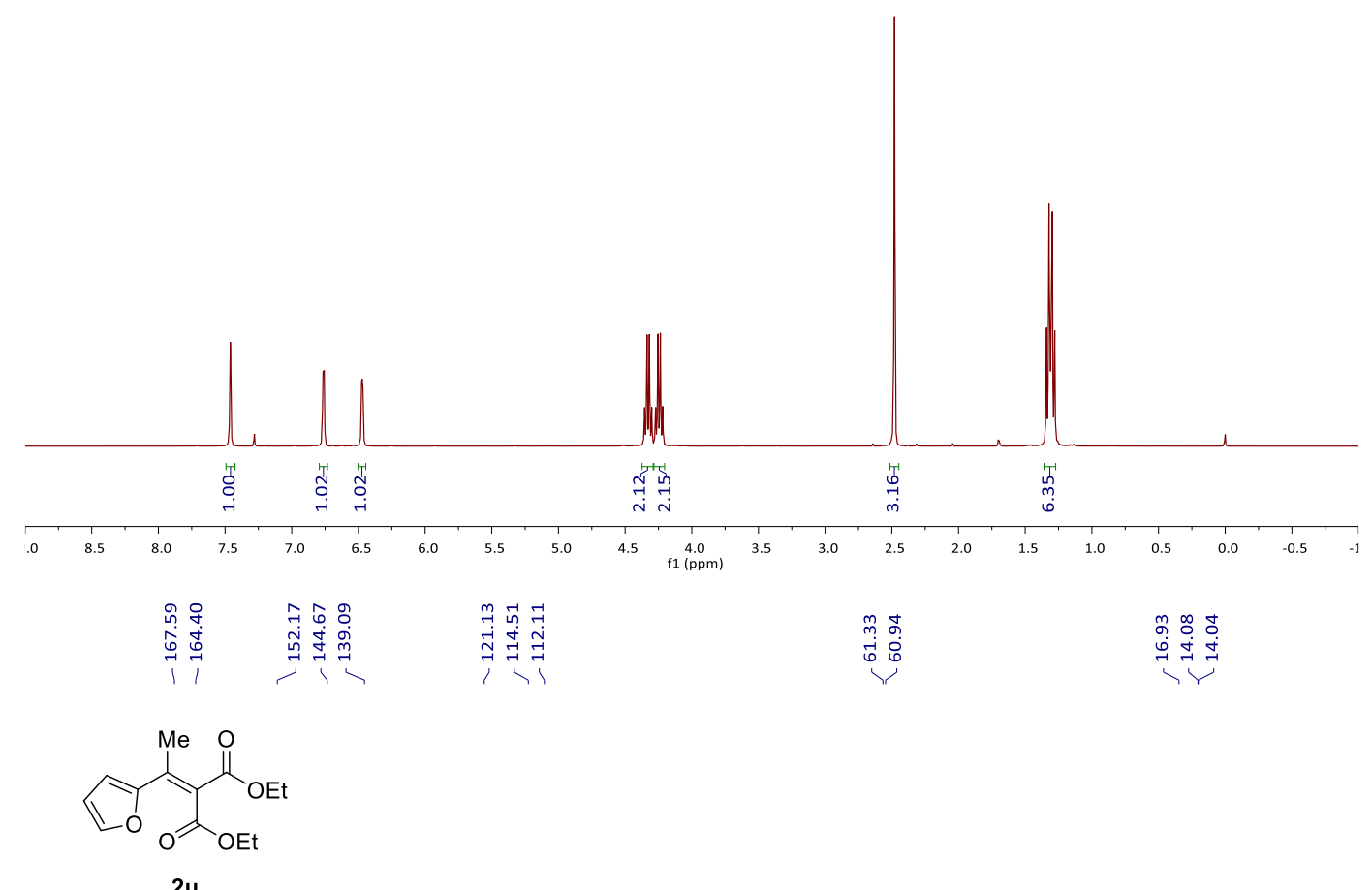

$\left({ }^{13} \mathrm{C} \mathrm{NMR}, 101 \mathrm{MHz}, \mathrm{CDCl}_{3}\right)$

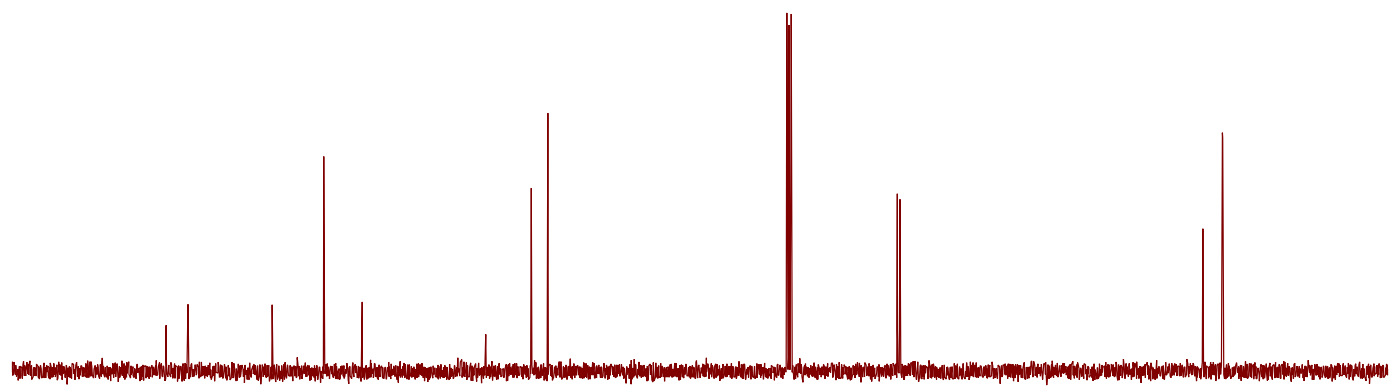

\begin{tabular}{lllllllllllllllllllllllll}
\hline 90 & 180 & 170 & 160 & 150 & 140 & 130 & 120 & 110 & 100 & 90 & 80 & 70 & 60 & 50 & 40 & 30 & 20 & 10 & 0 & $-:$
\end{tabular} 
Diethyl-2-(1-cyclohexylethylidene)malonate (2v)

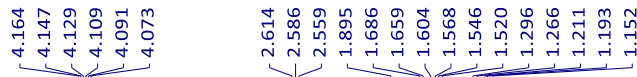

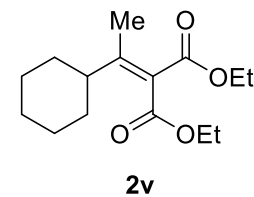

$\left({ }^{1} \mathrm{H} \mathrm{NMR}, 400 \mathrm{MHz}, \mathrm{CDCl}_{3}\right)$

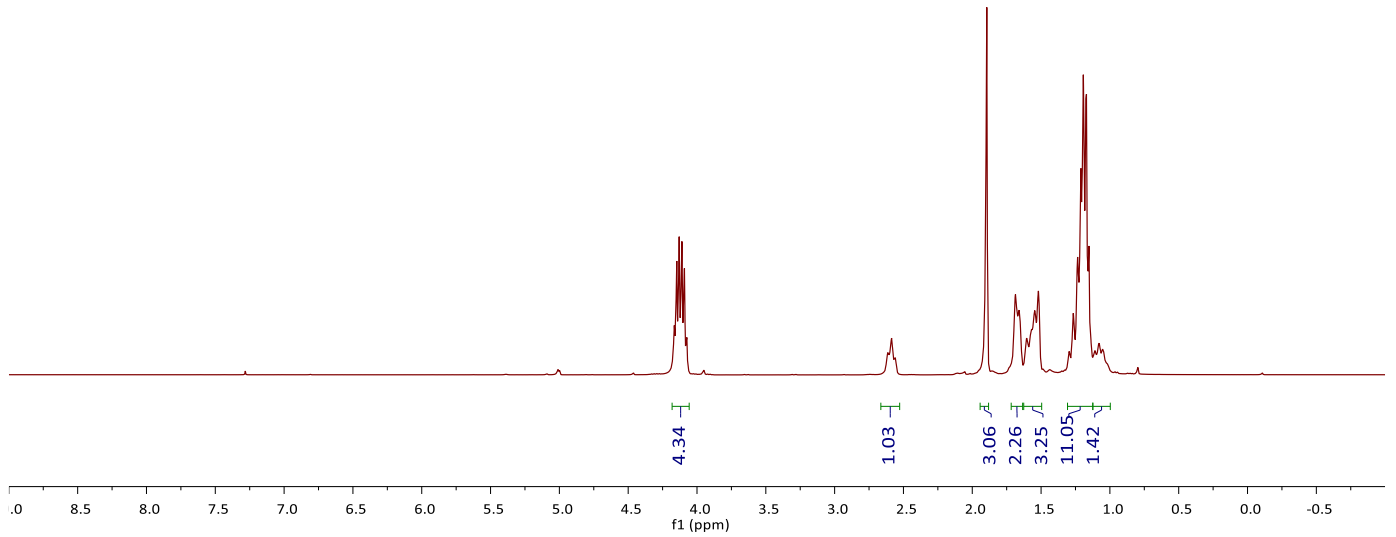

๓ุํา

突突过

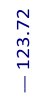

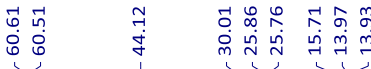

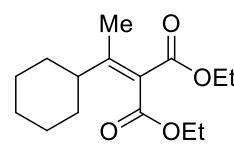

2v

$\left({ }^{13} \mathrm{C} \mathrm{NMR}, 101 \mathrm{MHz}, \mathrm{CDCl}_{3}\right)$

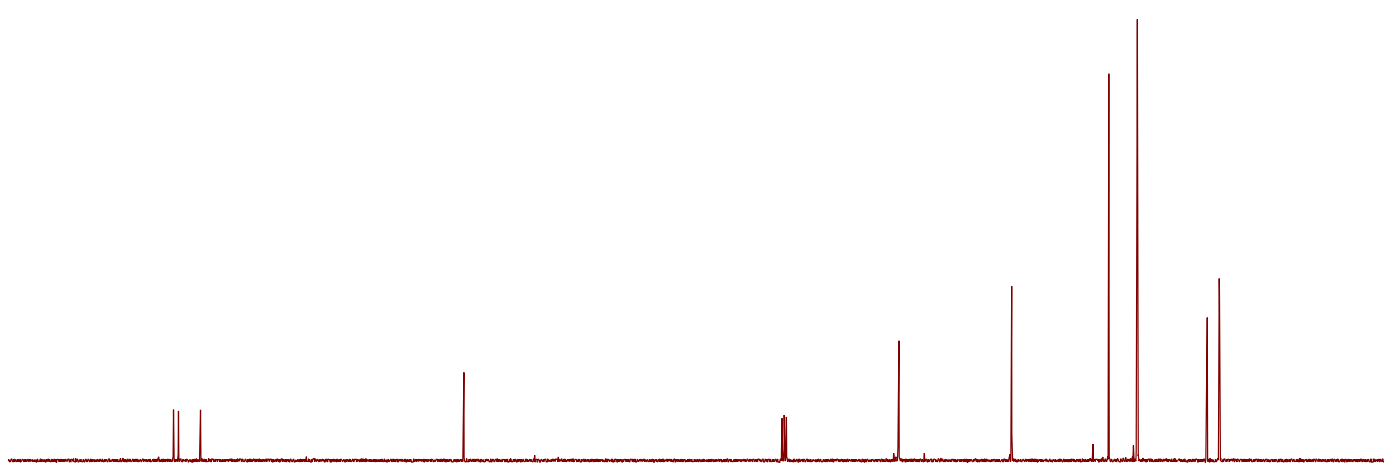

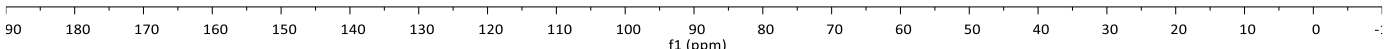


(-)-(R)-Diethyl-2-(1-phenylethyl)malonate (3a)

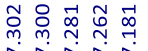

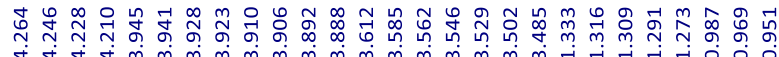

in

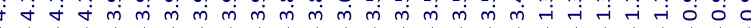<smiles>CCOC(=O)C(C(=O)OCC)C(=O)c1ccccc1</smiles>

$3 a$

$\left({ }^{1} \mathrm{H} \mathrm{NMR}, 400 \mathrm{MHz}, \mathrm{CDCl}_{3}\right)$

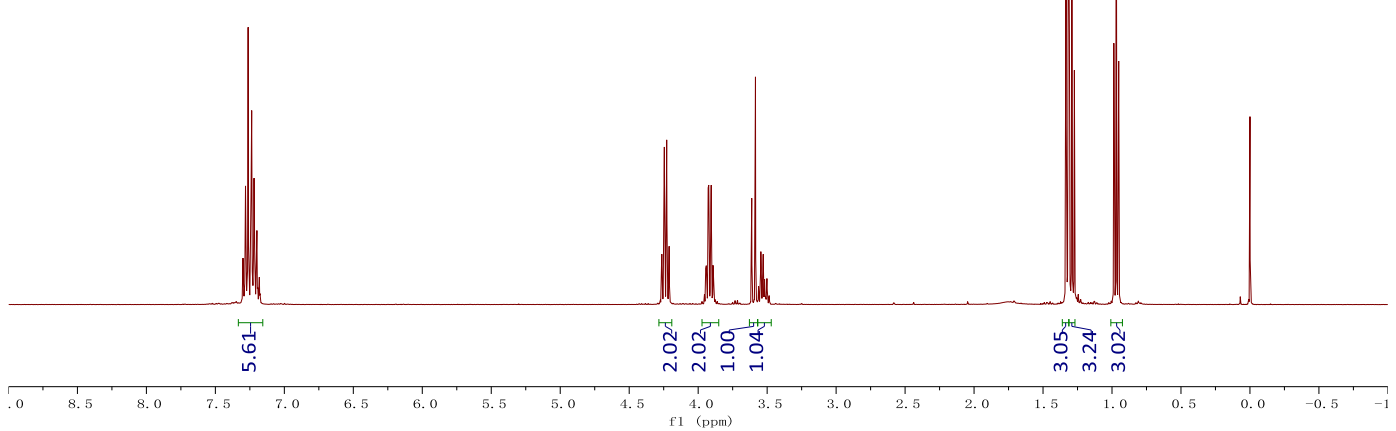

(-)-Diethyl 2-(1-phenylpropyl)malonate (3b)

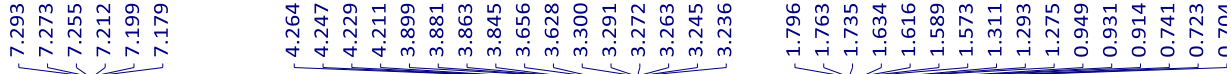<smiles>CC=C(c1ccccc1)C(C(=O)OCC)C(=O)OCC</smiles>

3b

$\left({ }^{1} \mathrm{H} \mathrm{NMR}, 400 \mathrm{MHz}, \mathrm{CDCl}_{3}\right)$

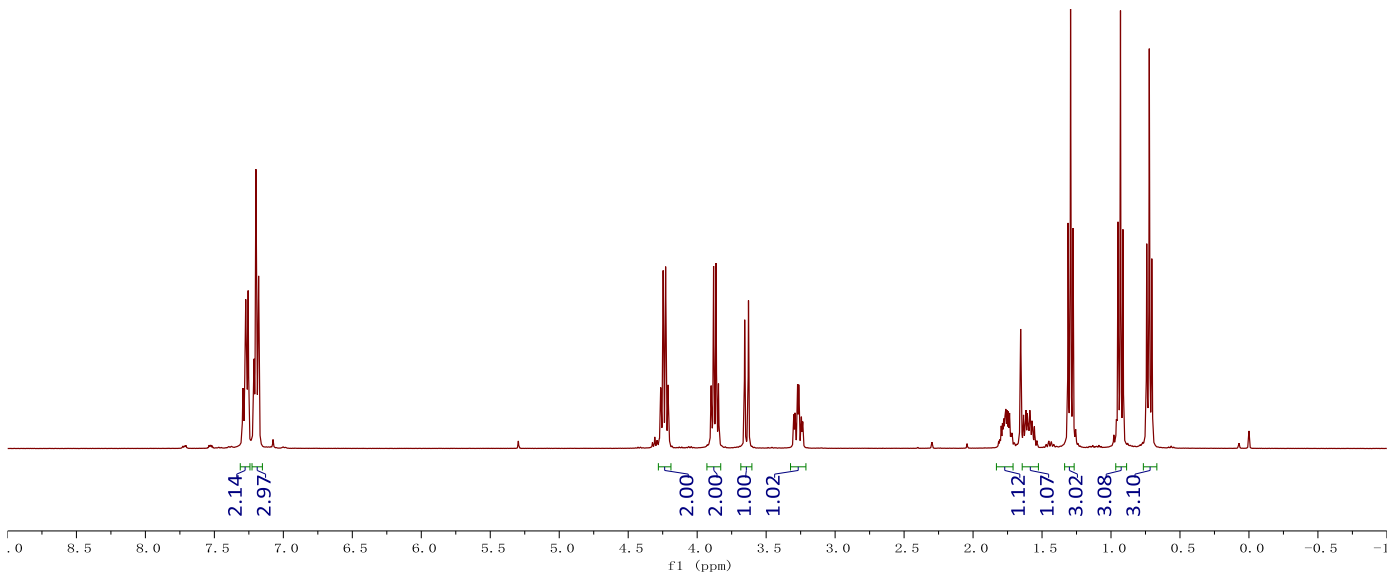


(-)-Diethyl 2-(1-phenylbutyl)malonate (3c)

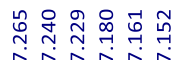

inis<smiles>CCOC(=O)C(C(=O)OCC)C(C(=O)OCC)c1ccccc1</smiles>

$3 \mathrm{c}$

( $\left.{ }^{1} \mathrm{H} \mathrm{NMR}, 400 \mathrm{MHz}, \mathrm{CDCl}_{3}\right)$
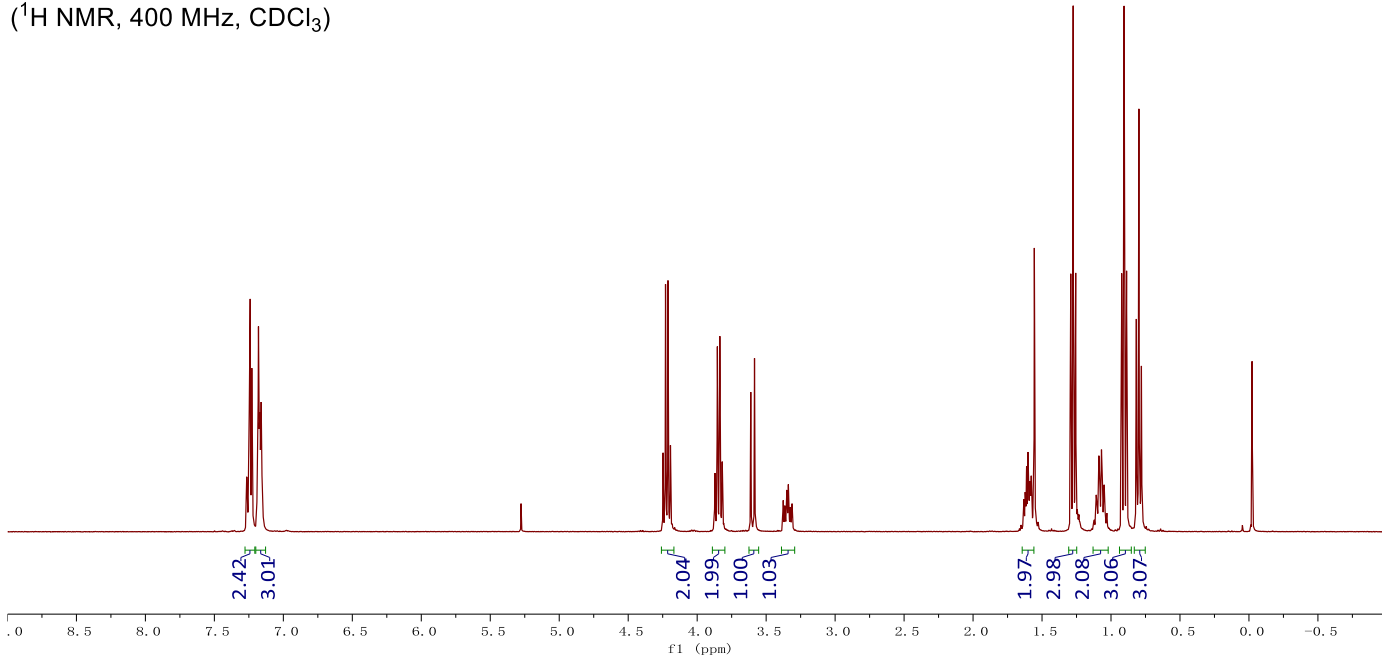

\section{(-)-(R)-Diethyl-2-(1-(4-fluorophenyl)ethyl)malonate (3d)}

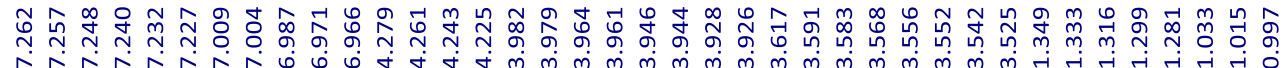

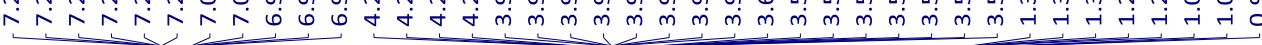

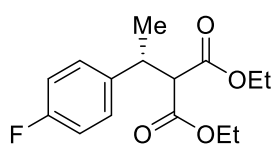

$(R)-3 \mathbf{d}$

$\left({ }^{1} \mathrm{H} \mathrm{NMR}, 400 \mathrm{MHz}, \mathrm{CDCl}_{3}\right)$

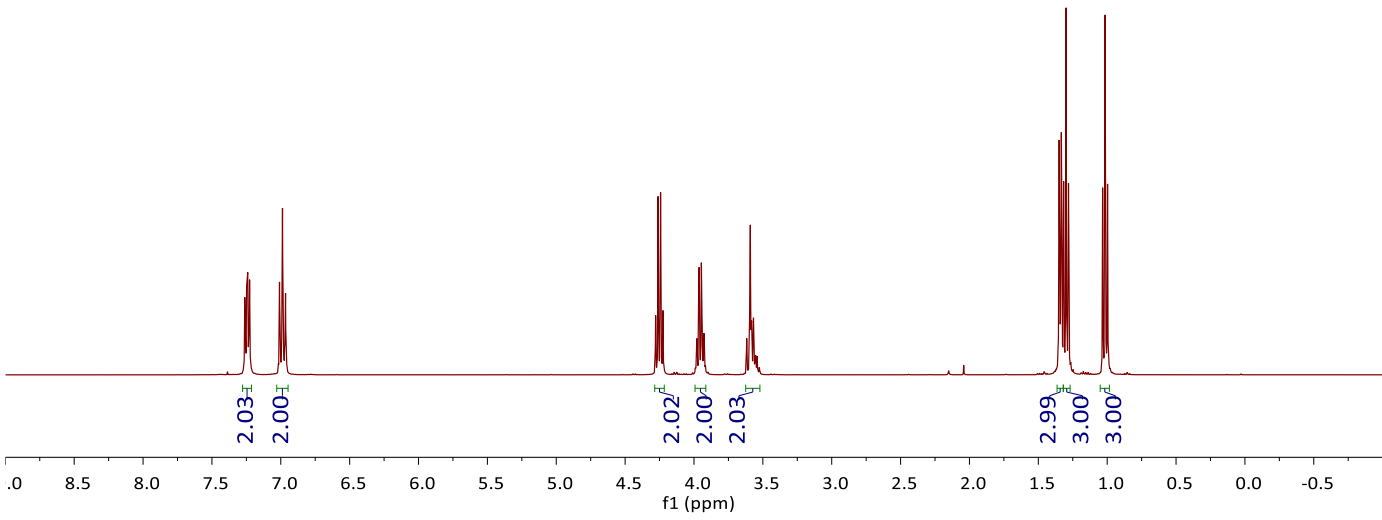




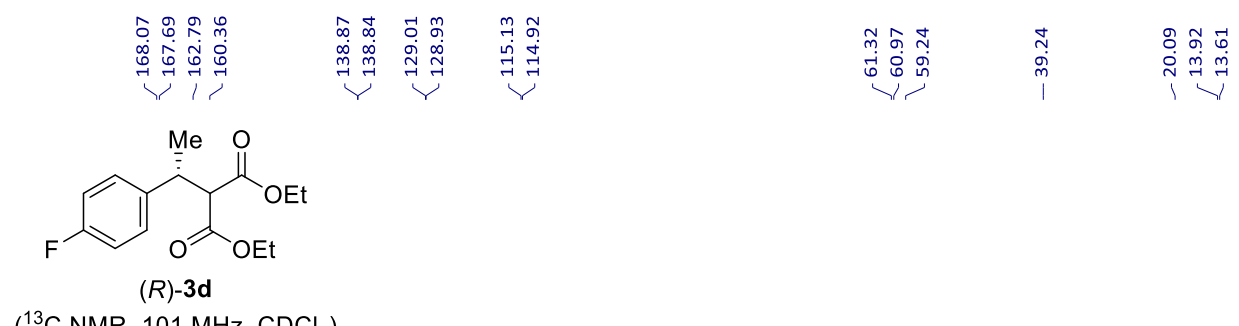

$\left({ }^{13} \mathrm{C} \mathrm{NMR}, 101 \mathrm{MHz}, \mathrm{CDCl}_{3}\right)$
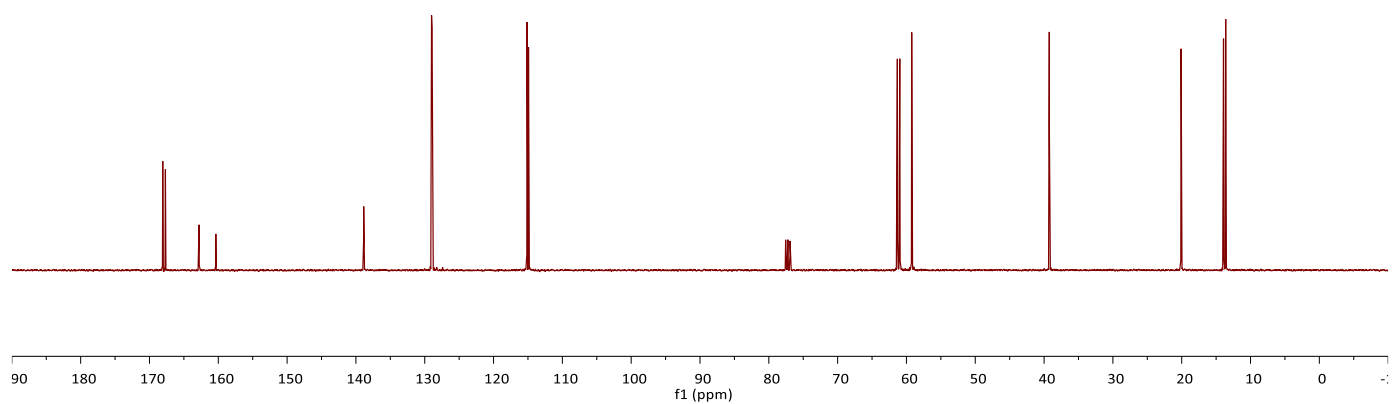

(-)-Diethyl 2-(1-(4-chlorophenyl)ethyl)malonate (3e)

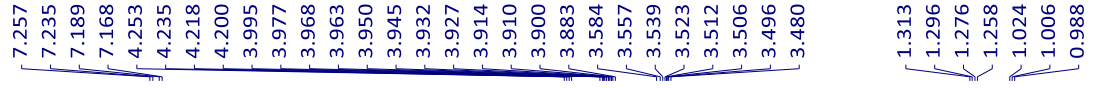

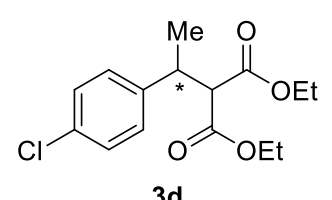

( $\left.{ }^{1} \mathrm{H} \mathrm{NMR}, 400 \mathrm{MHz}, \mathrm{CDCl}_{3}\right)$

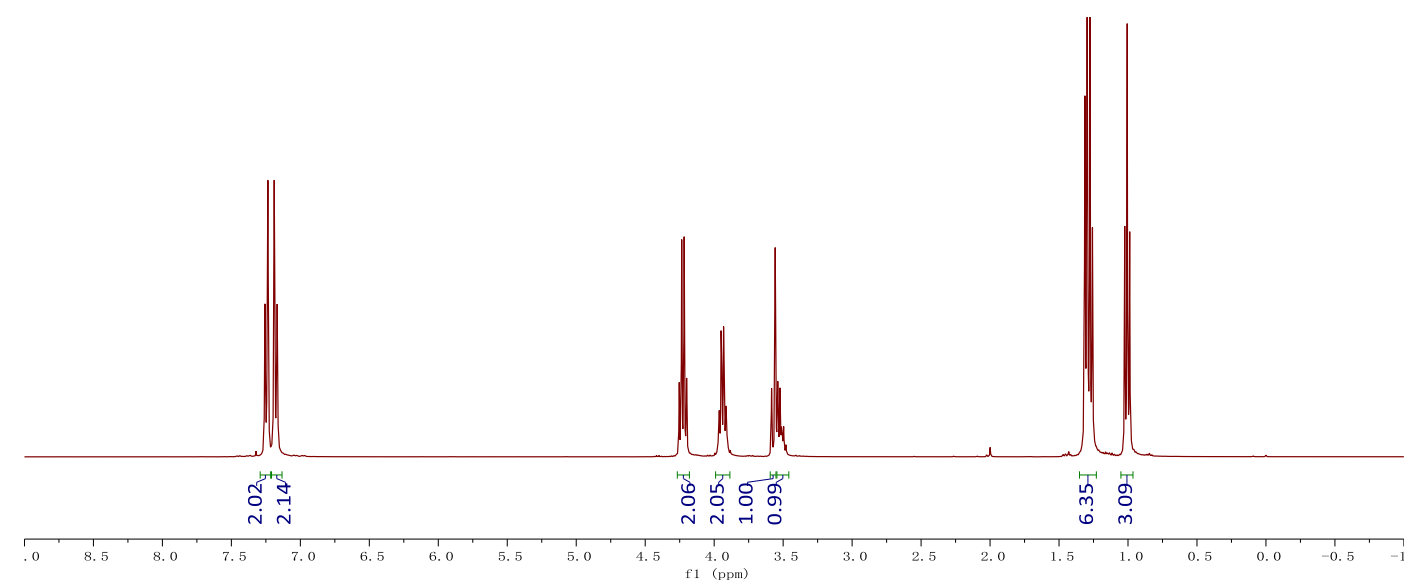


(-)-Diethyl-2-(1-(4-bromophenyl)ethyl)malonate (3f)
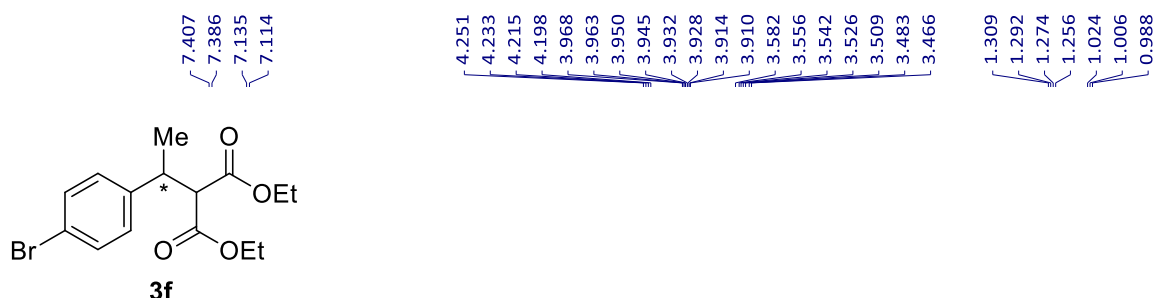

$\left({ }^{1} \mathrm{H}\right.$ NMR, $\left.400 \mathrm{MHz}, \mathrm{CDCl}_{3}\right)$

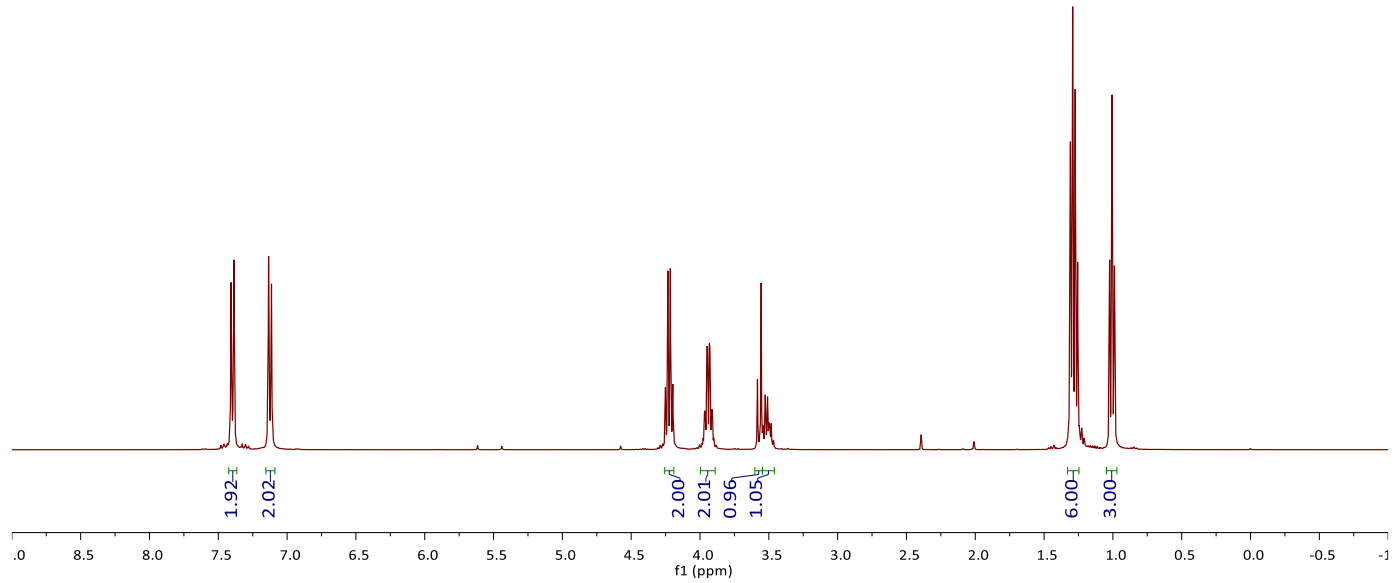

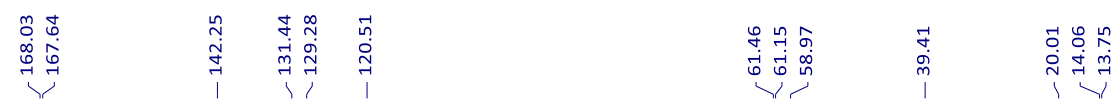

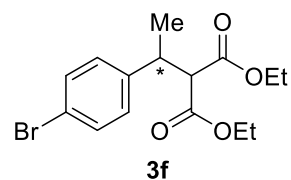

$\left({ }^{13} \mathrm{C}\right.$ NMR, $\left.101 \mathrm{MHz}, \mathrm{CDCl}_{3}\right)$

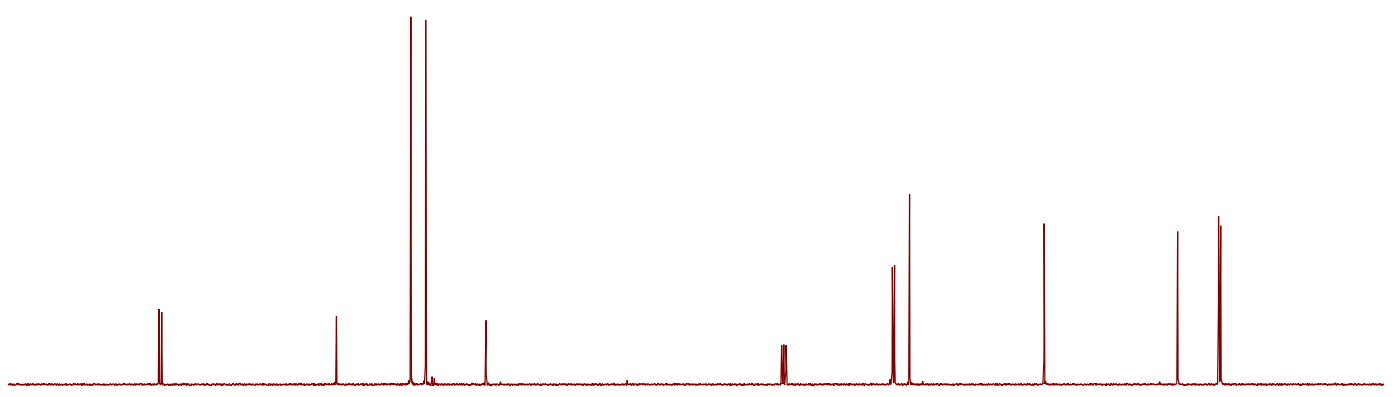

\begin{tabular}{lllllllllllllllllllllllll}
\hline 90 & 180 & 170 & 160 & 150 & 140 & 130 & 120 & 110 & 100 & 90 & 80 & 70 & 60 & 50 & 40 & 30 & 20 & 10 & 0 & $-:$
\end{tabular} 
(-)-(R)-Diethyl-2-(1-(4-(trifluoromethyl)phenyl)ethyl)malonate (3g)

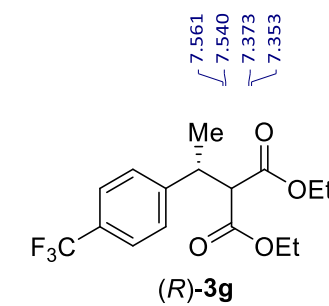

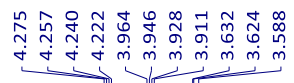

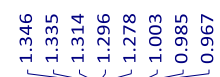

$\left({ }^{1} \mathrm{H} \mathrm{NMR}, 400 \mathrm{MHz}, \mathrm{CDCl}_{3}\right)$

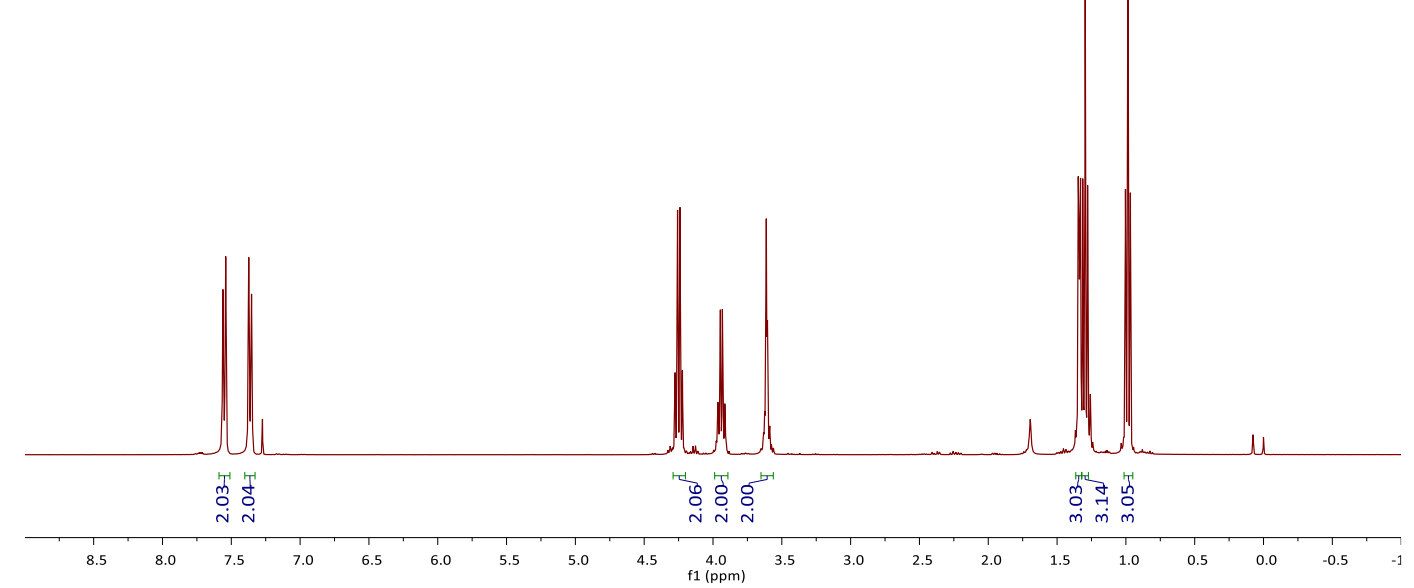

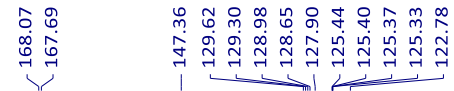

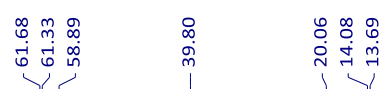

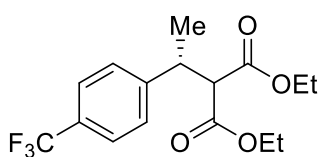

(R)-3 $\mathbf{g}$

$\left({ }^{13} \mathrm{C} \mathrm{NMR}, 101 \mathrm{MHz}, \mathrm{CDCl}_{3}\right)$

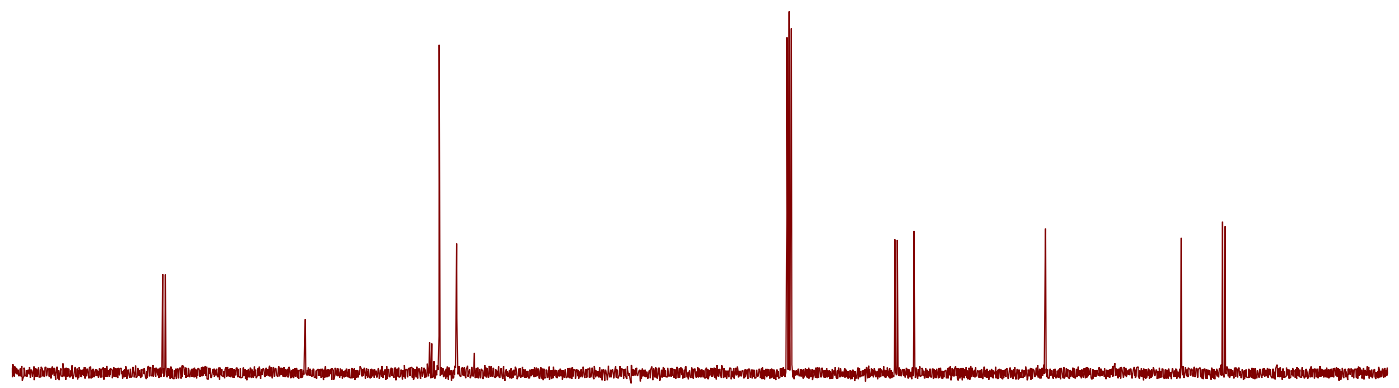

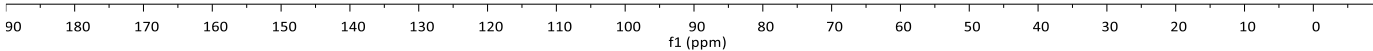




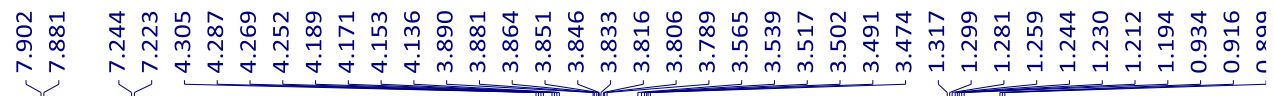<smiles>CCOC(=O)c1ccc(C(C)C(C(=O)OCC)C(=O)OCC)cc1</smiles>

$\left({ }^{1} \mathrm{H}\right.$ NMR, $\left.400 \mathrm{MHz}, \mathrm{CDCl}_{3}\right)$
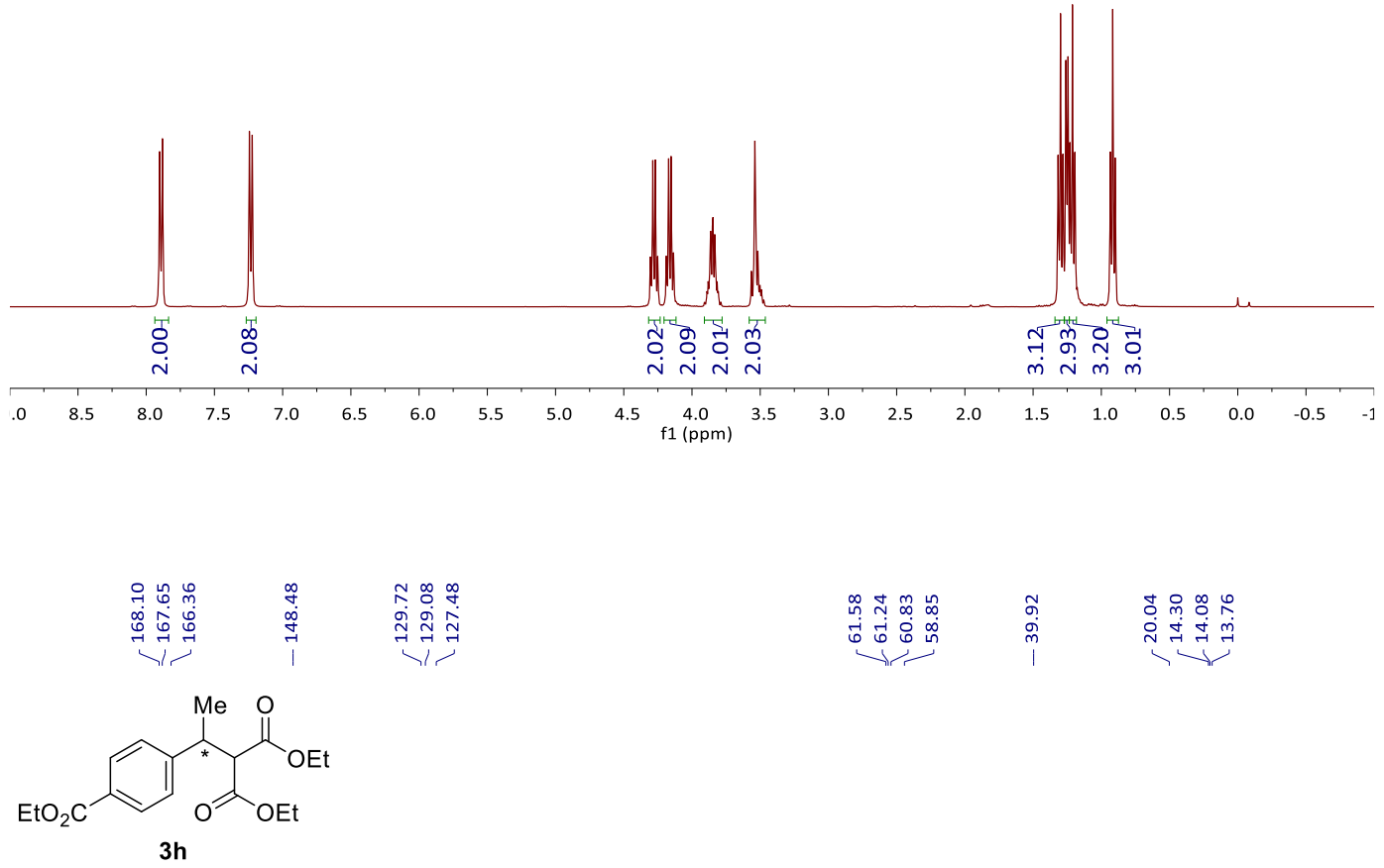

\section{象}

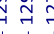

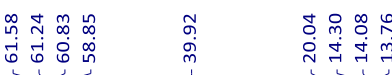

$\left({ }^{13} \mathrm{C} \mathrm{NMR}, 101 \mathrm{MHz}, \mathrm{CDCl}_{3}\right)$

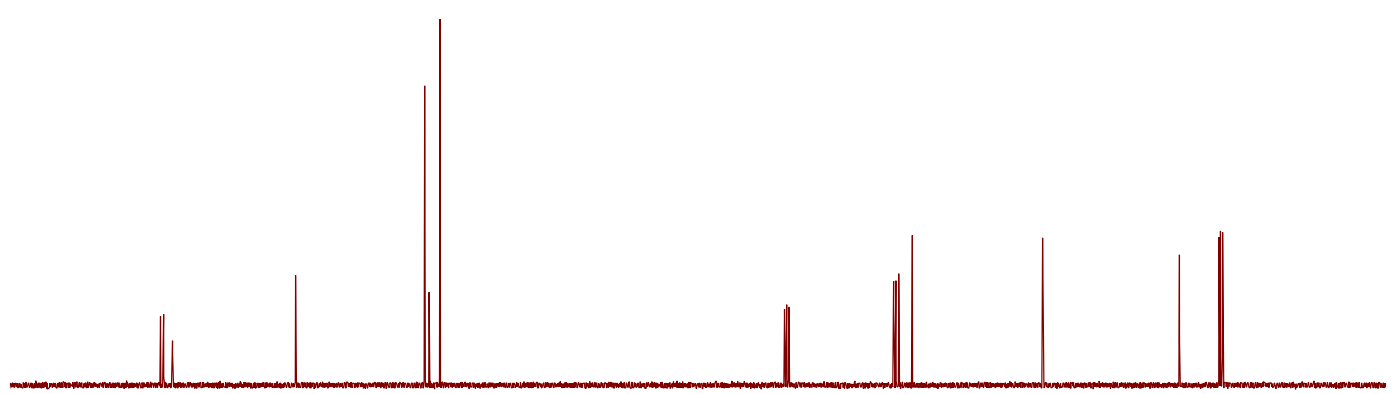

$90 \quad 180$

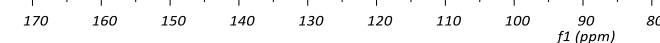


(-)-(R)-Diethyl-2-(1-([1,1'-biphenyl]-4-yl)ethyl)malonate (3i)

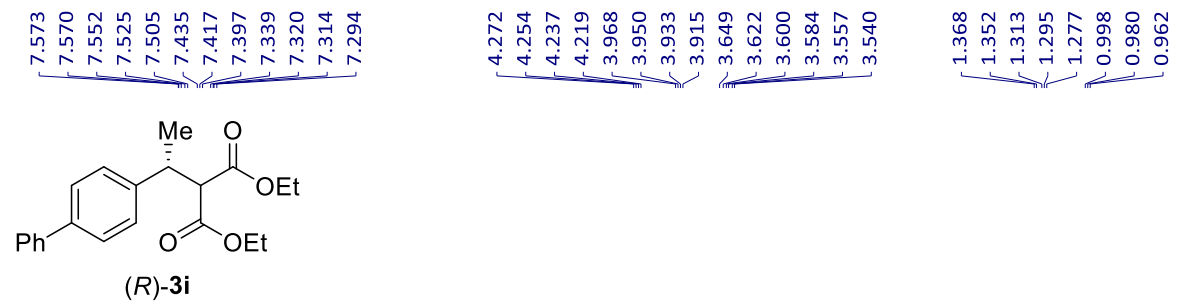

$\left({ }^{1} \mathrm{H} \mathrm{NMR}, 400 \mathrm{MHz}, \mathrm{CDCl}_{3}\right)$

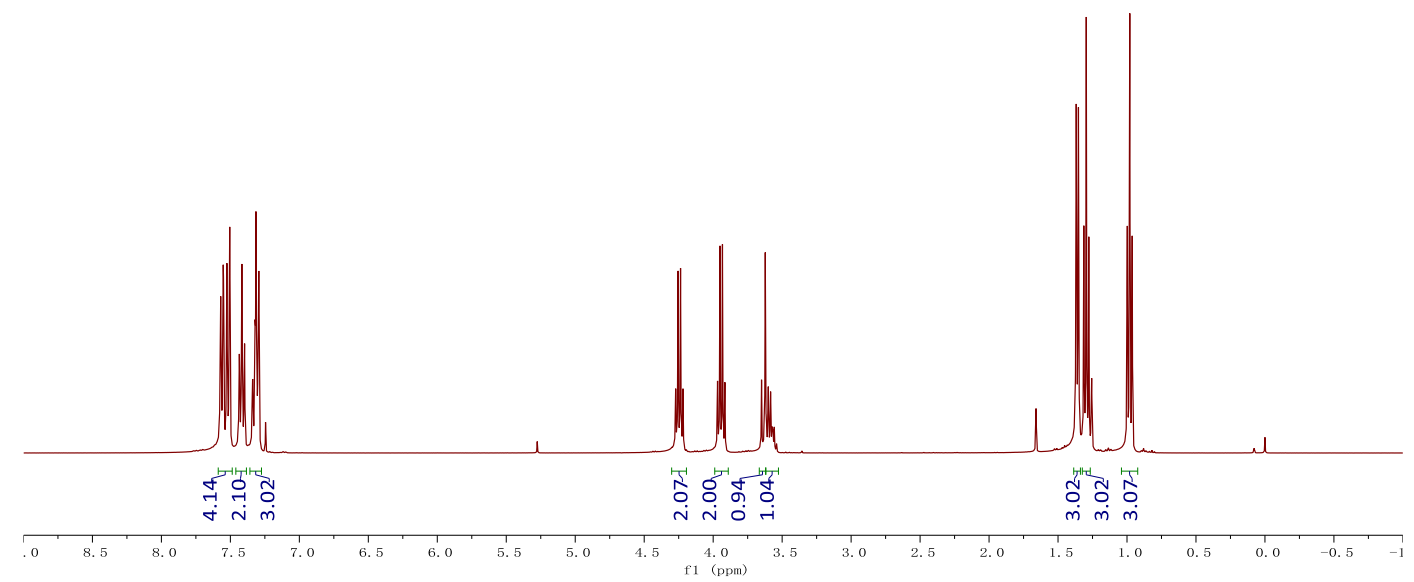

(-)-Diethyl 2-(1-(p-tolyl)ethyl)malonate (3j)

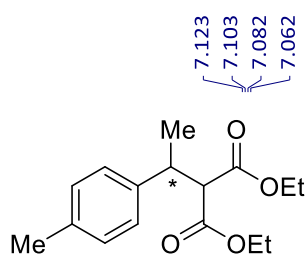

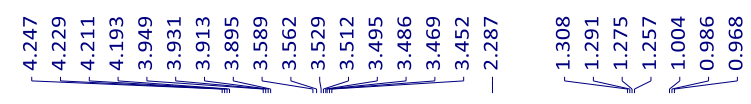

$\left({ }^{1} \mathrm{H} \mathrm{NMR}, 400 \mathrm{MHz}, \mathrm{CDCl}_{3}\right)$

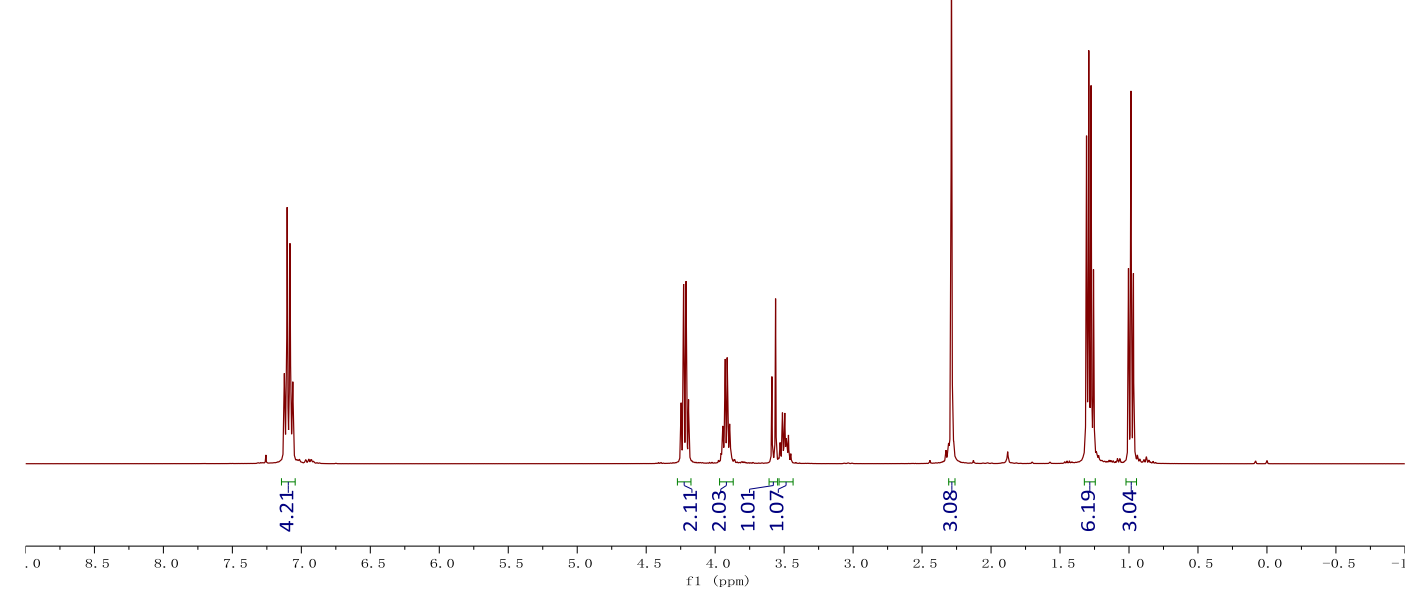


(-)-(R)-Diethyl-2-(1-(4-methoxyphenyl)ethyl)malonate (3k)

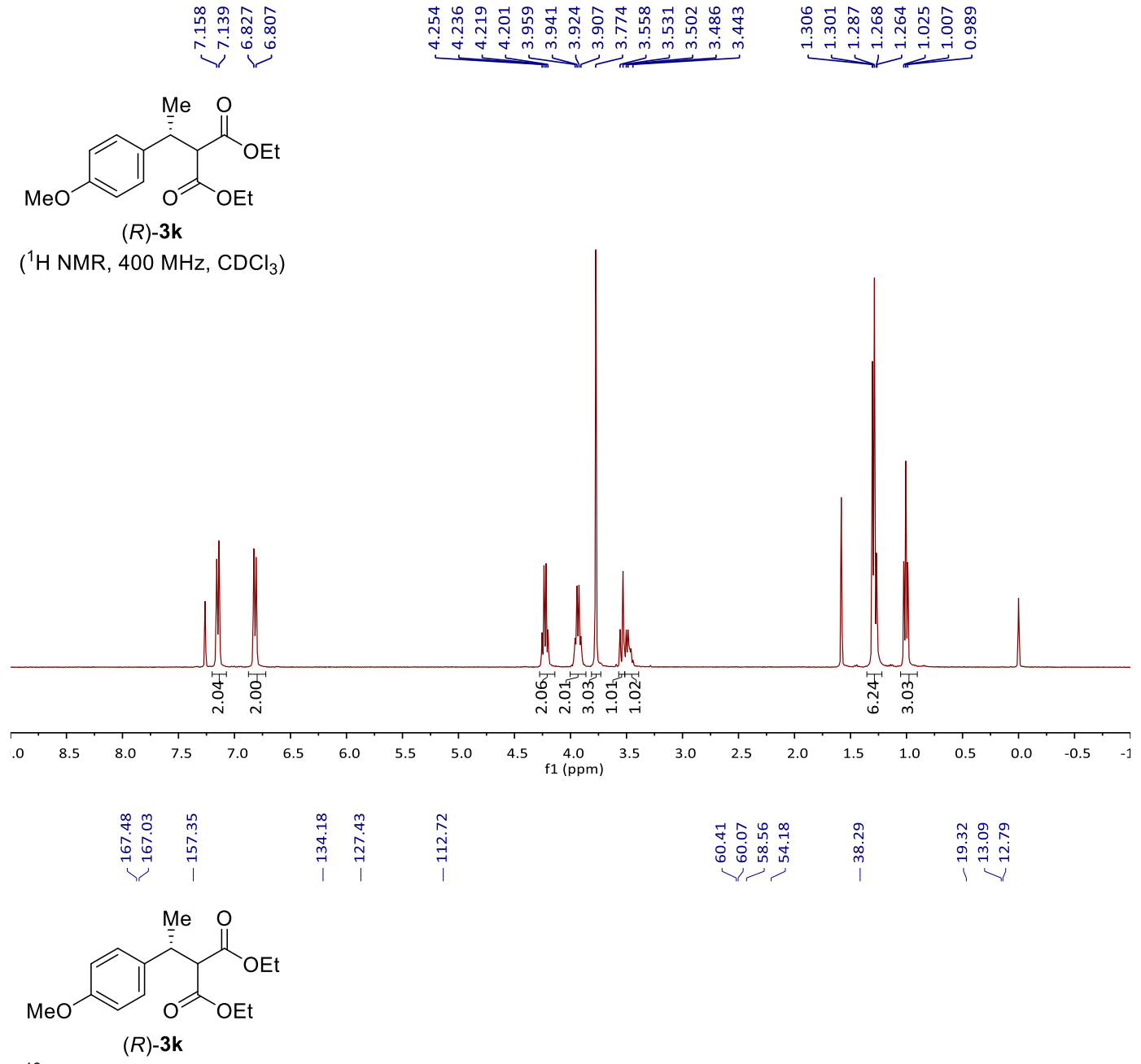

$\left({ }^{13} \mathrm{C} \mathrm{NMR}, 101 \mathrm{MHz}, \mathrm{CDCl}_{3}\right)$

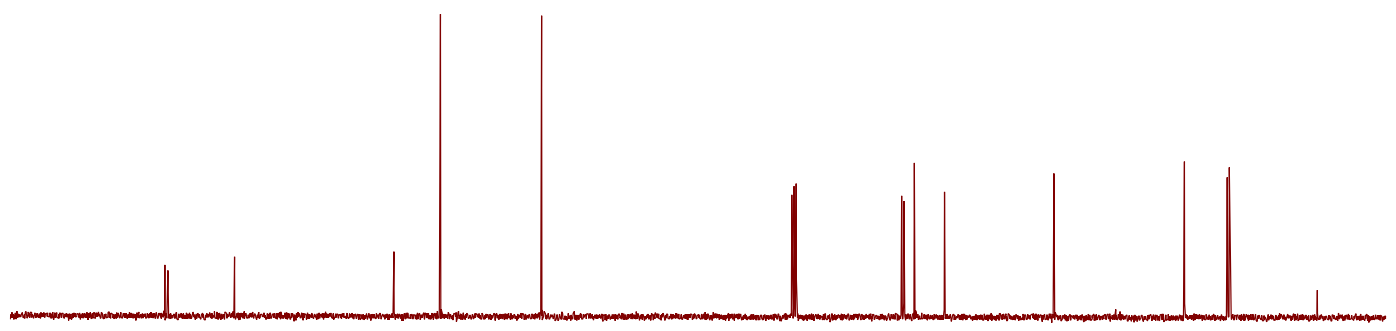

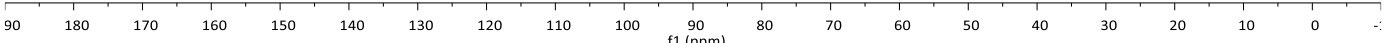


(-)-Diethyl-2-(1-(3-fluorophenyl)ethyl)malonate (3I)

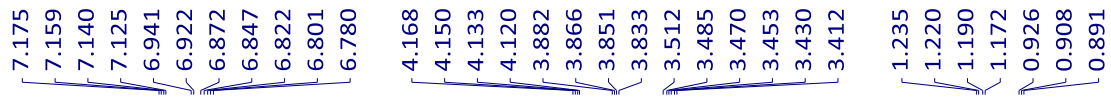

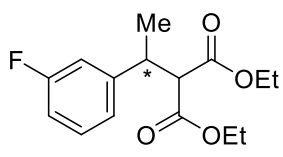

3)

$\left({ }^{1} \mathrm{H} \mathrm{NMR}, 400 \mathrm{MHz}, \mathrm{CDCl}_{3}\right)$

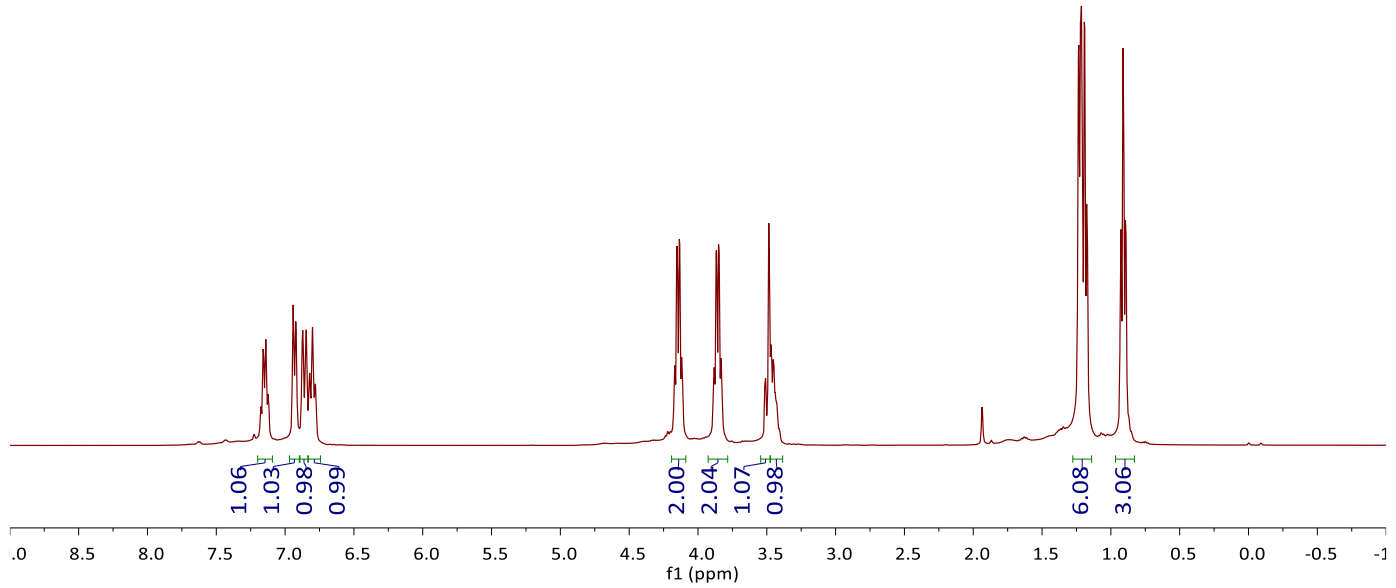

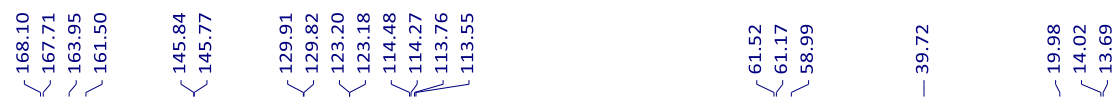

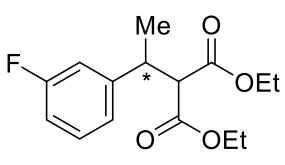

3I

$\left({ }^{13} \mathrm{C} \mathrm{NMR}, 101 \mathrm{MHz}, \mathrm{CDCl}_{3}\right)$

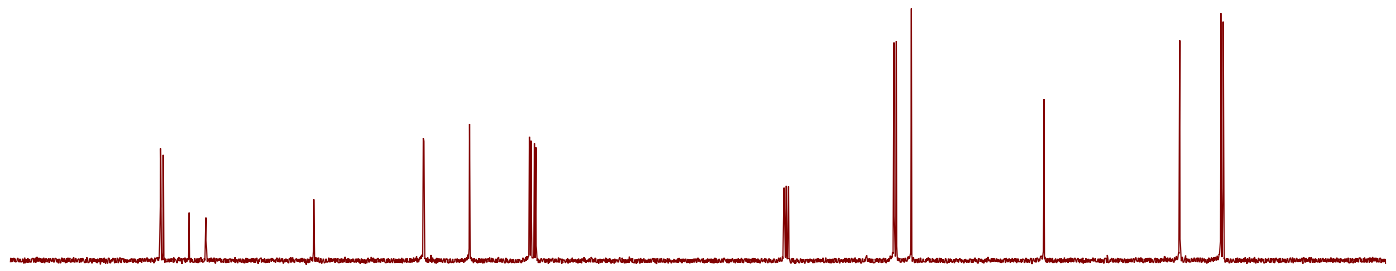

\begin{tabular}{rllllllllllllllllllll}
\hline 90 & 180 & 170 & 160 & 150 & 140 & 130 & 120 & 110 & 100 & $\begin{array}{c}90 \\
\mathrm{f} 1(\mathrm{ppm})\end{array}$ & 80 & 70 & 60 & 50 & 40 & 30 & 20 & 10 & 0 & $-:$
\end{tabular} 
(-)-Diethyl-2-(1-(m-tolyl)ethyl)malonate (3m)

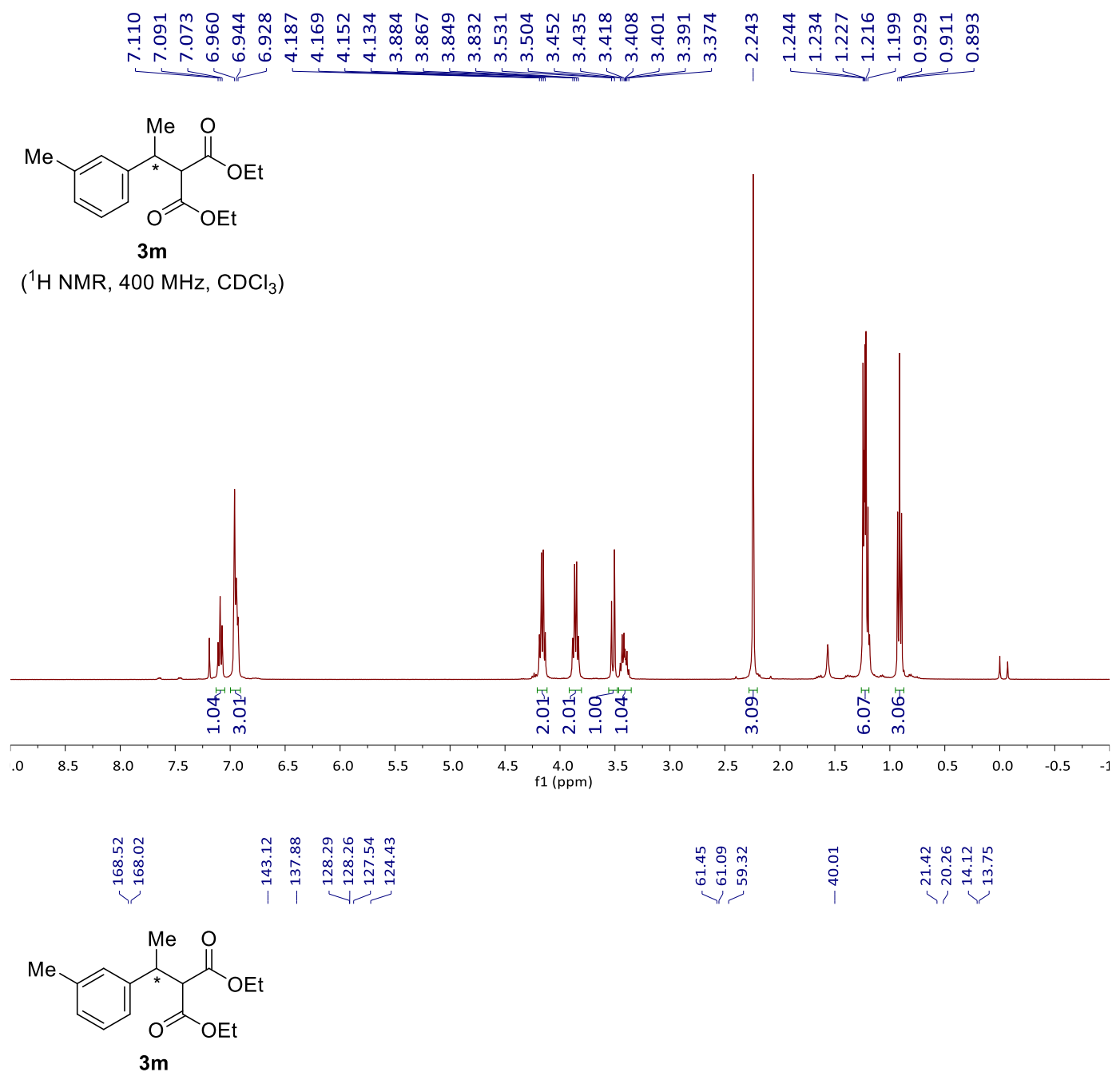

$\left({ }^{13} \mathrm{C} \mathrm{NMR}, 101 \mathrm{MHz}, \mathrm{CDCl}_{3}\right)$

$100 \underset{\substack{90 \\ \text { f1 (ppm) }}}{10}$ 
(-)-Diethyl-2-(1-(3-methoxyphenyl)ethyl)malonate (3n)
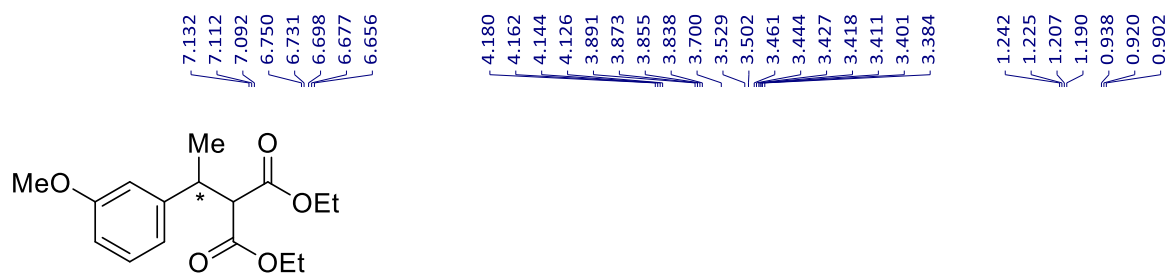

$3 n$

$\left({ }^{1} \mathrm{H}\right.$ NMR, $\left.400 \mathrm{MHz}, \mathrm{CDCl}_{3}\right)$

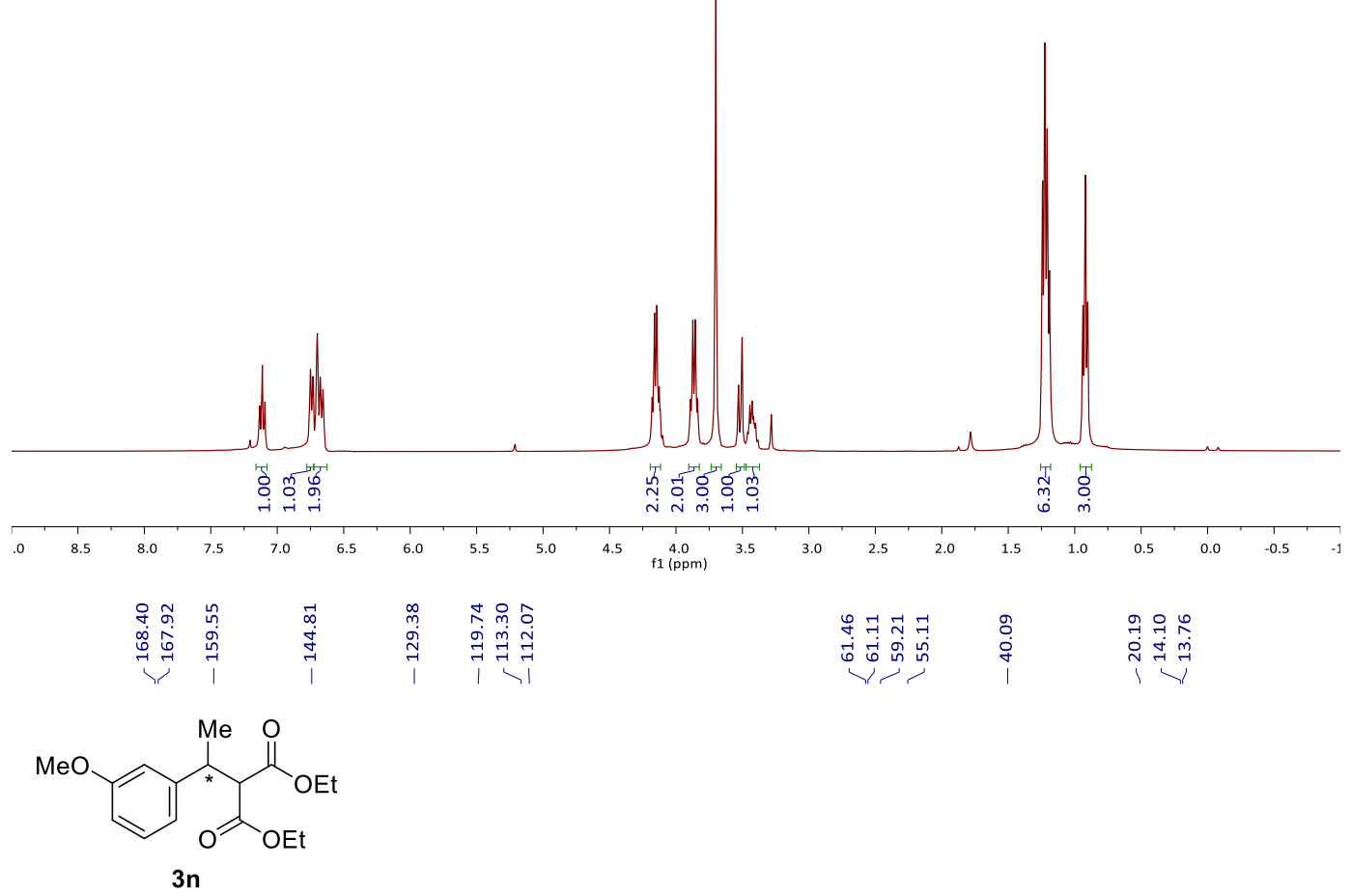

$\left({ }^{13} \mathrm{C} \mathrm{NMR}, 101 \mathrm{MHz}, \mathrm{CDCl}_{3}\right)$

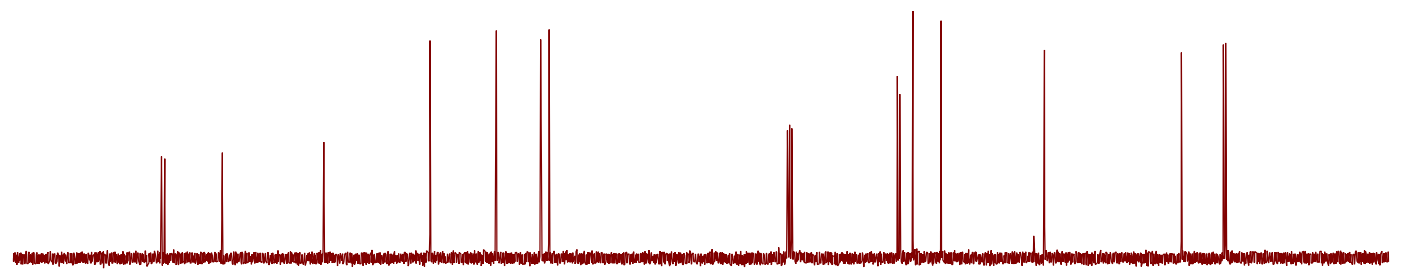

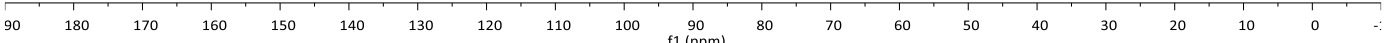


(-)-Diethyl-2-(1-(2-fluorophenyl)ethyl)malonate (3o)

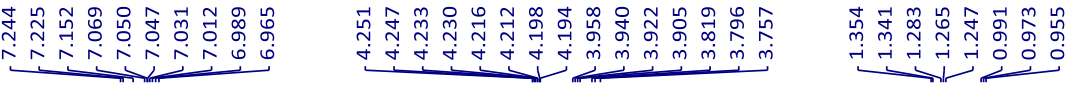

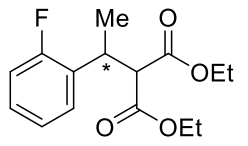

30

( ${ }^{1} \mathrm{H} \mathrm{NMR}, 400 \mathrm{MHz}, \mathrm{CDCl}_{3}$ )

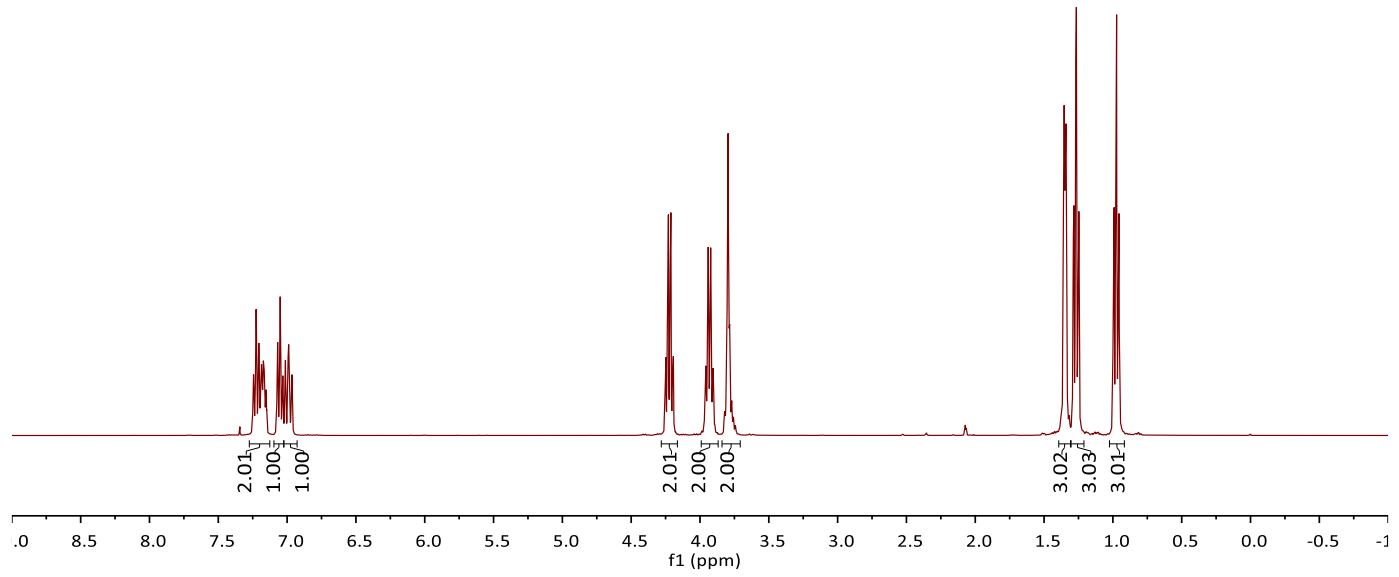

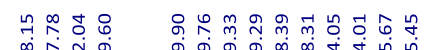

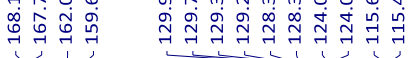

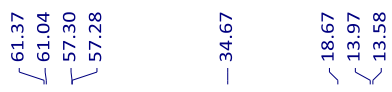<smiles>CCOC(=O)C(C(=O)OCC)C(C)c1ccccc1F</smiles>

30

$\left({ }^{13} \mathrm{C} \mathrm{NMR}, 101 \mathrm{MHz}, \mathrm{CDCl}_{3}\right)$

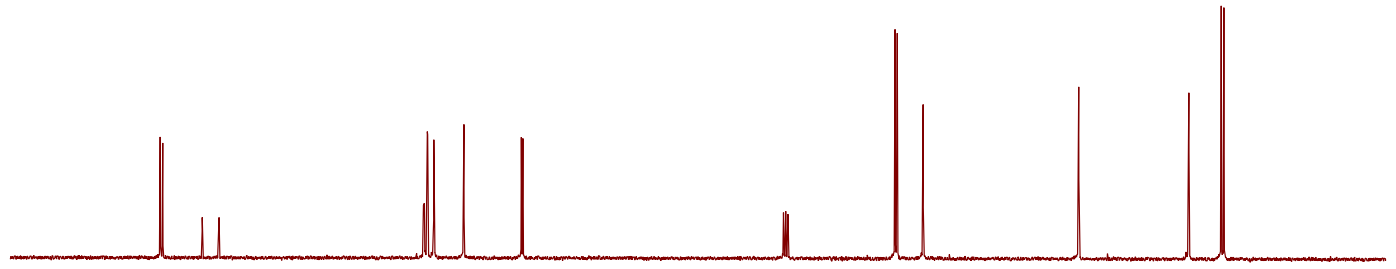

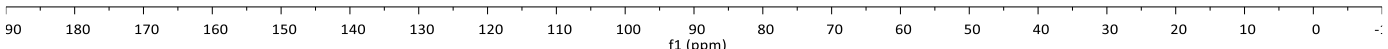


(-)-Diethyl-2-(1-(o-tolyl)ethyl)malonate (3p)

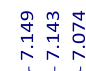

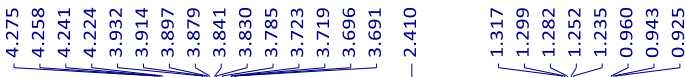

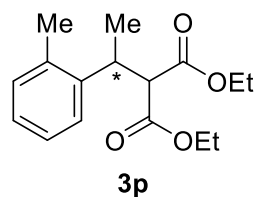

$\left({ }^{1} \mathrm{H}\right.$ NMR, $\left.400 \mathrm{MHz}, \mathrm{CDCl}_{3}\right)$

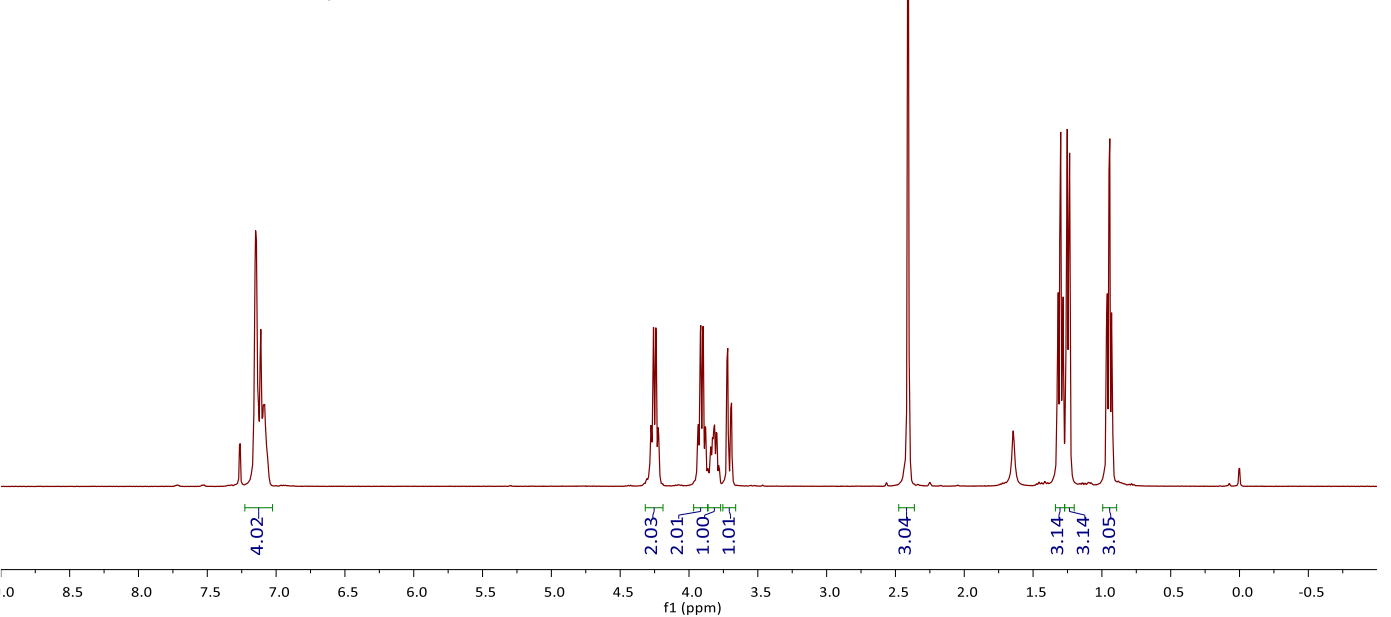

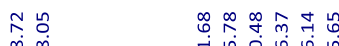

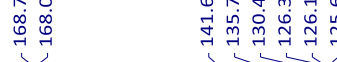

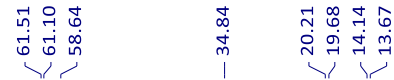

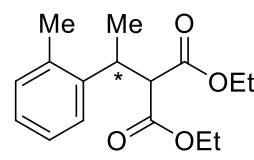

3p

$\left({ }^{13} \mathrm{C}\right.$ NMR, $\left.101 \mathrm{MHz}, \mathrm{CDCl}_{3}\right)$

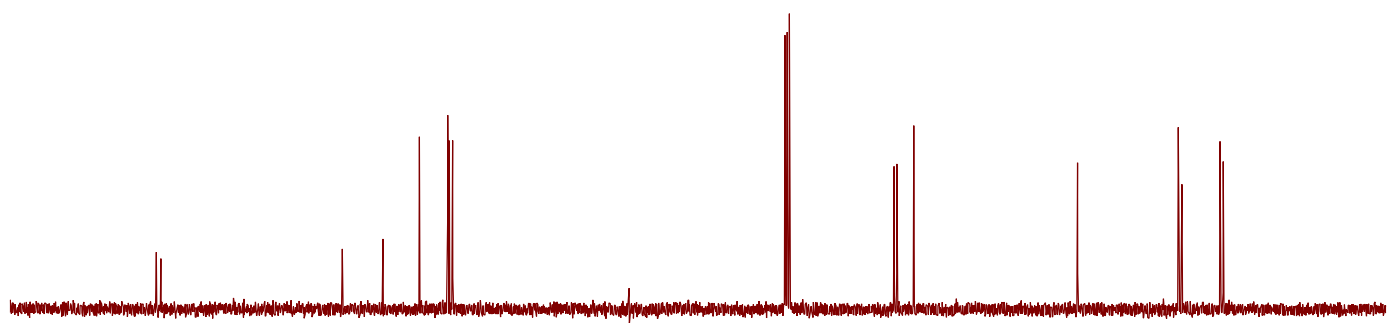

$\begin{array}{lllllllllllllllllllllll} & 90 & 180 & 170 & 160 & 150 & 140 & 130 & 120 & 110 & 100 & 90 & 80 & 70 & 60 & 50 & 40 & 30 & 20 & 10 & 0 & -\vdots\end{array}$ 
(-)-(R)-Diethyl-2-(1-(benzo[d][1,3]dioxol-5-yl)ethyl)malonate (3q)

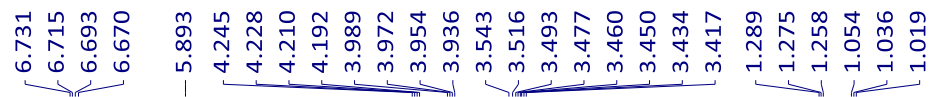

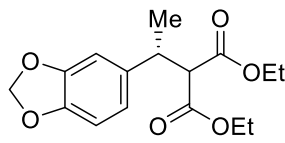

$(R)-3 q$

( ${ }^{1} \mathrm{H} \mathrm{NMR}, 400 \mathrm{MHz}, \mathrm{CDCl}_{3}$ )

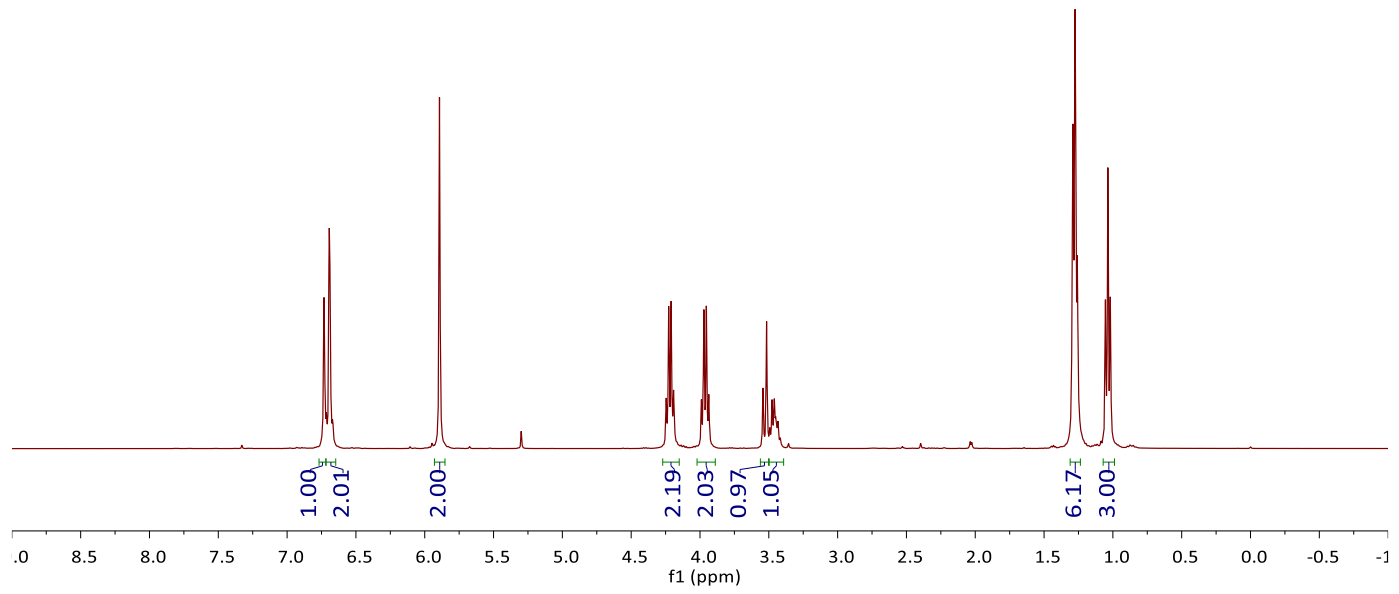

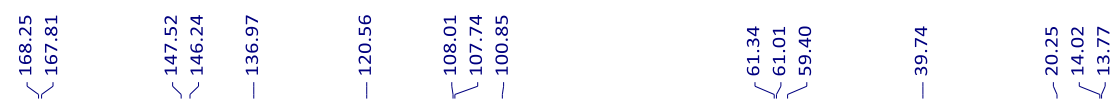

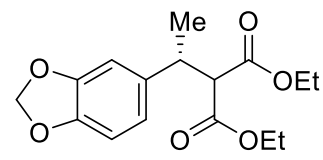

$(R)-3 q$

$\left({ }^{13} \mathrm{C} \mathrm{NMR}, 101 \mathrm{MHz}, \mathrm{CDCl}_{3}\right)$

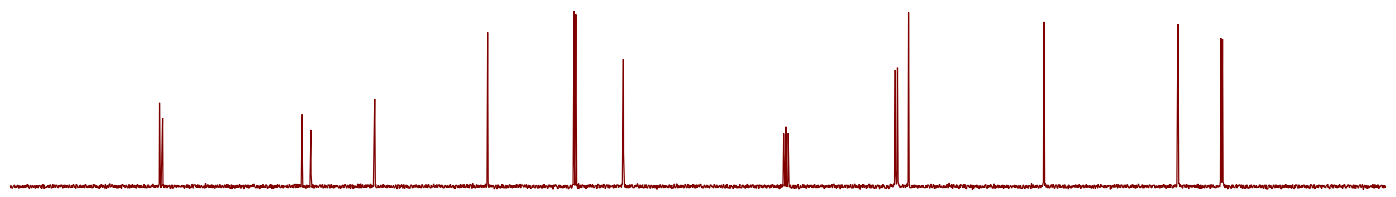

\begin{tabular}{rllllllllllllllllllll}
\hline 90 & 180 & 170 & 160 & 150 & 140 & 130 & 120 & 110 & 100 & $\begin{array}{c}90 \\
\mathrm{f} 1(\mathrm{ppm})\end{array}$ & 80 & 70 & 60 & 50 & 40 & 30 & 20 & 10 & 0 & $-:$
\end{tabular} 
(+)-Diethyl-2-(1-(naphthalen-1-yl)ethyl)malonate (3r)
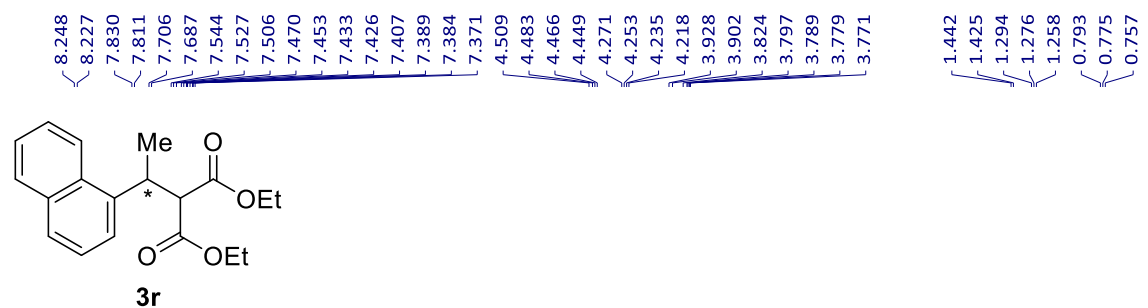

$\left({ }^{1} \mathrm{H} \mathrm{NMR}, 400 \mathrm{MHz}, \mathrm{CDCl}_{3}\right)$
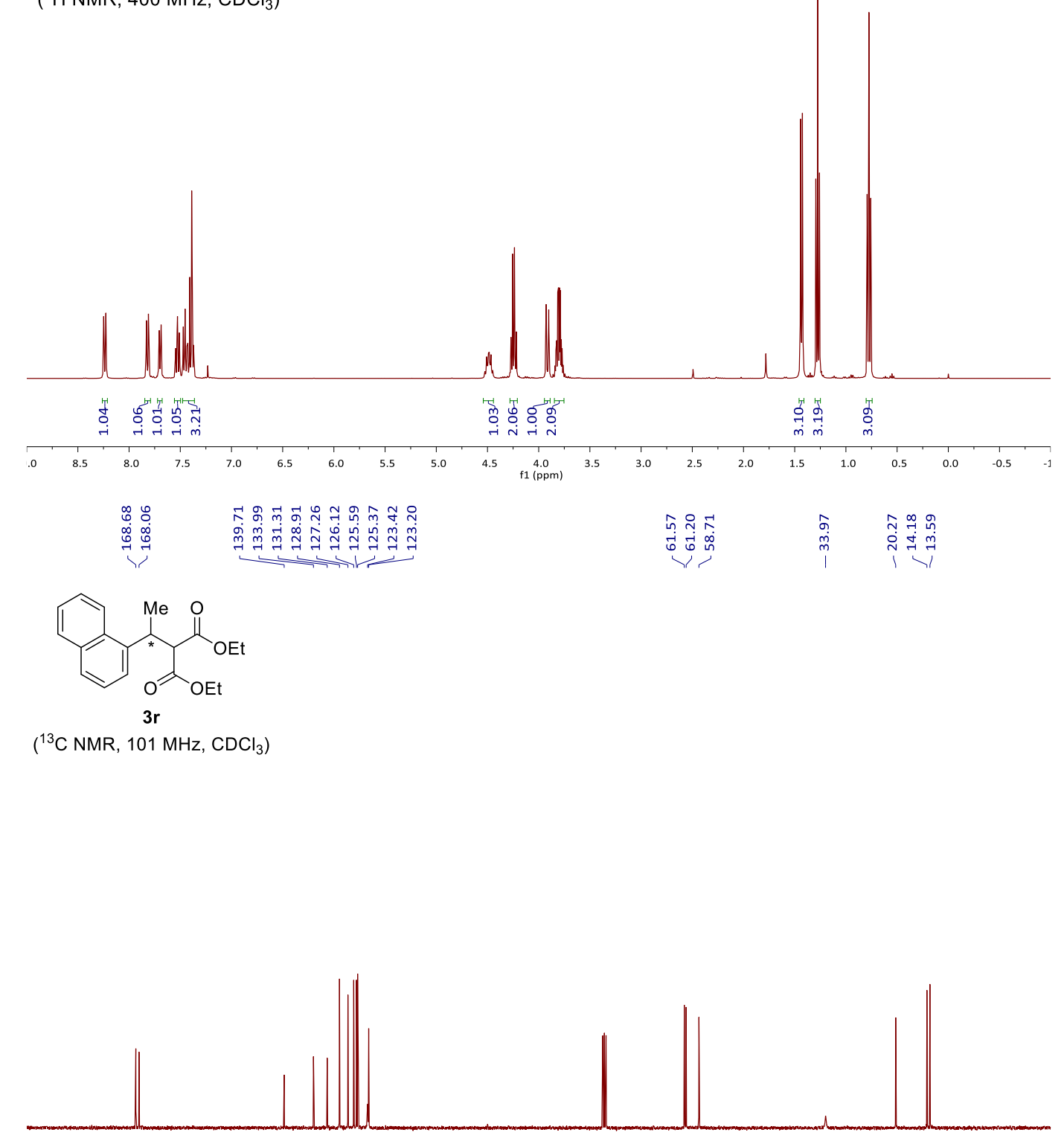

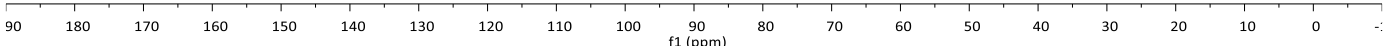


(-)-Diethyl-2-(1-(naphthalen-2-yl)ethyl)malonate (3s)

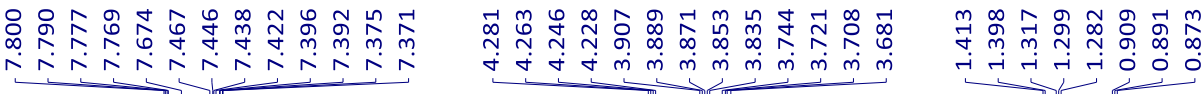

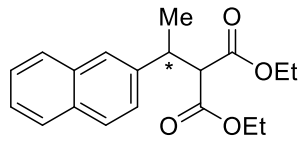

3s

$\left({ }^{1} \mathrm{H} \mathrm{NMR}, 400 \mathrm{MHz}, \mathrm{CDCl}_{3}\right)$

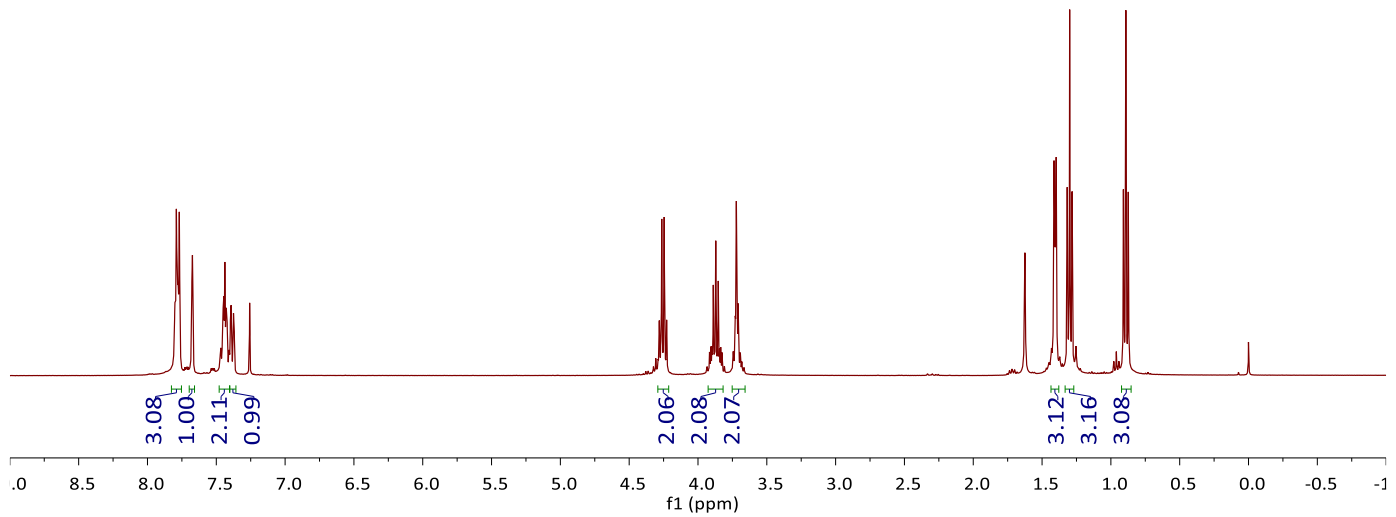

कo

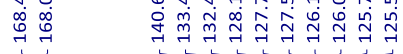

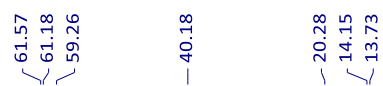

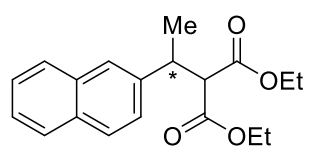

3s

$\left({ }^{13} \mathrm{C}\right.$ NMR, $\left.101 \mathrm{MHz}, \mathrm{CDCl}_{3}\right)$

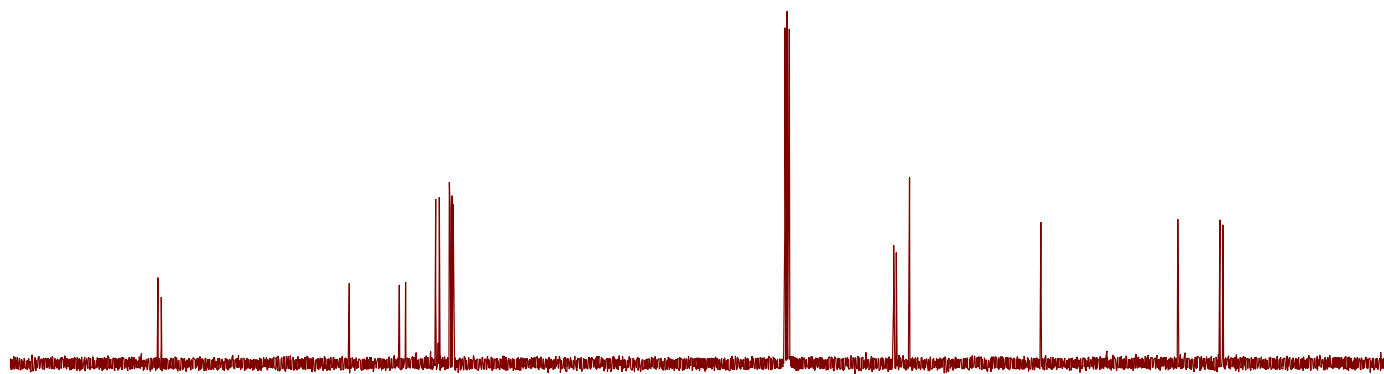

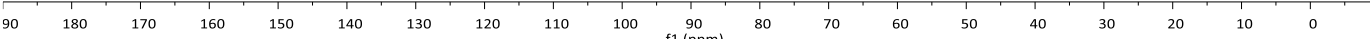


(-)-Diethyl-2-(1-(thiophen-2-yl)ethyl)malonate (3t)

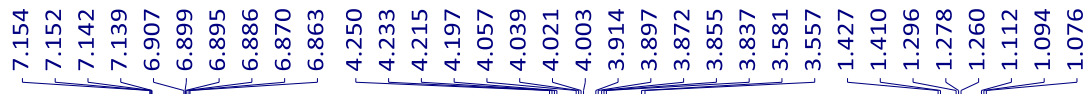

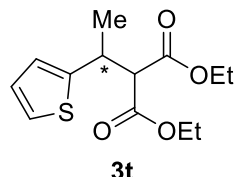

$\left({ }^{1} \mathrm{H}\right.$ NMR, $\left.400 \mathrm{MHz}, \mathrm{CDCl}_{3}\right)$

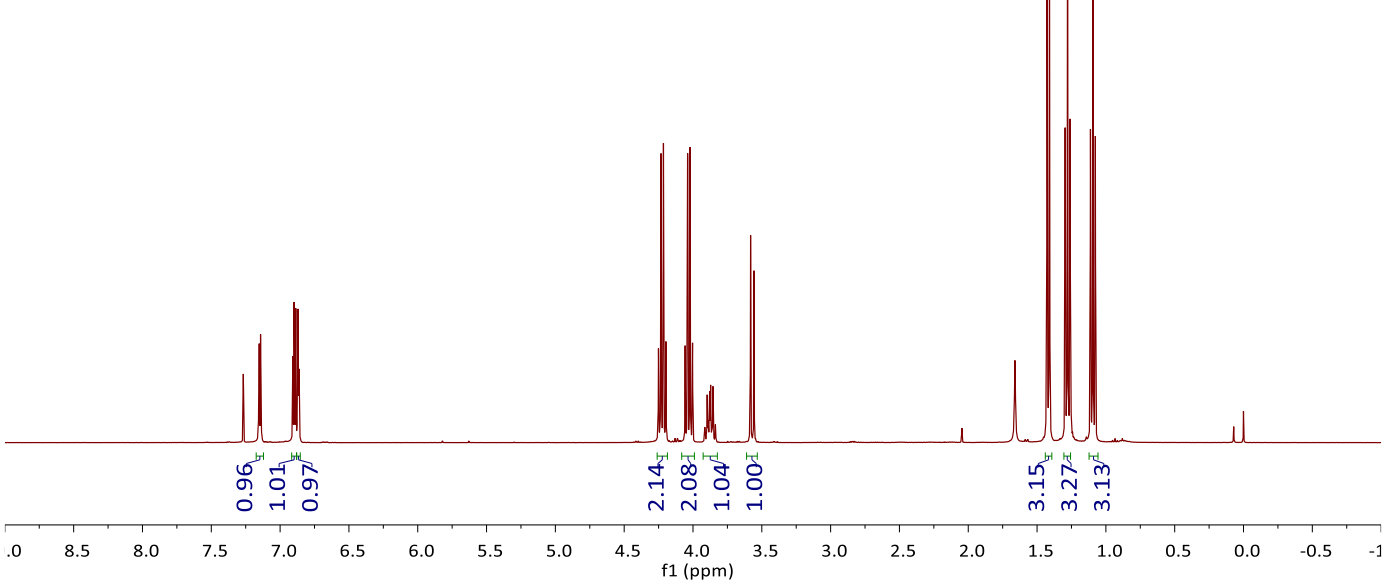

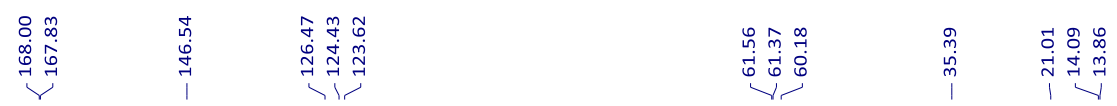

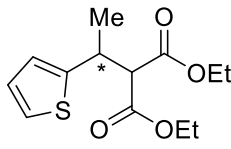

3t

$\left({ }^{13} \mathrm{C} \mathrm{NMR}, 101 \mathrm{MHz}, \mathrm{CDCl}_{3}\right)$

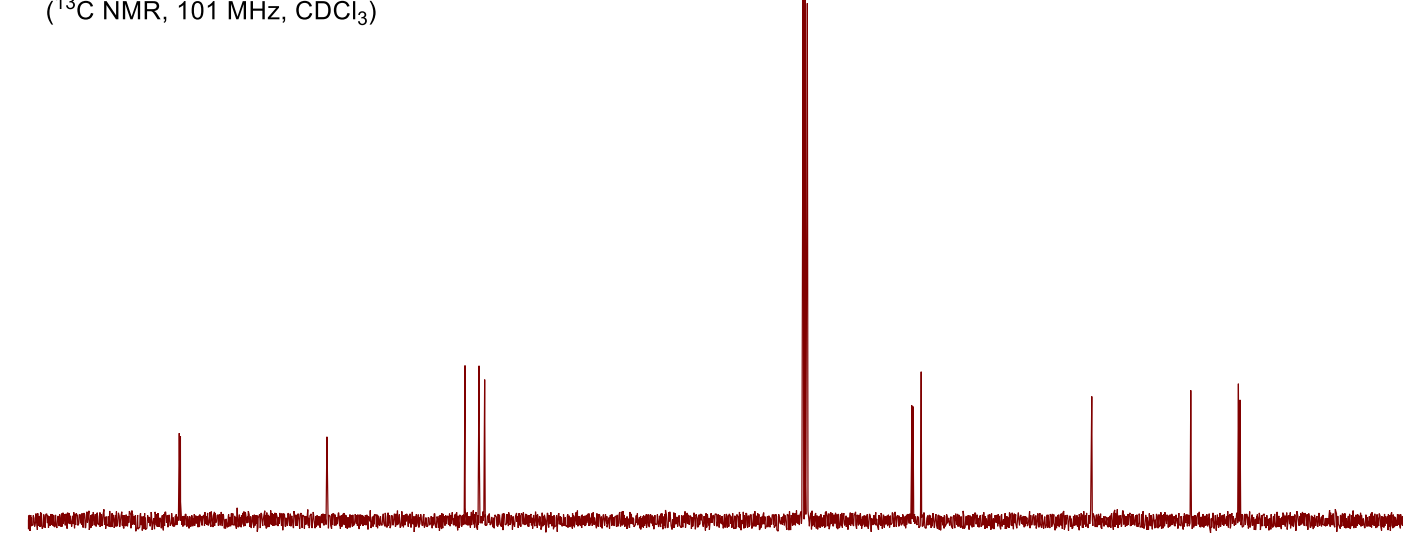

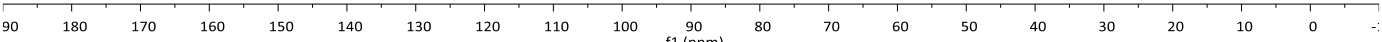


(-)-Diethyl 2-(1-(furan-2-yl)ethyl)malonate (3u)

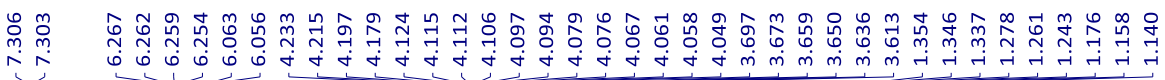

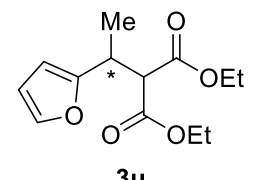

$3 \mathbf{u}$

$\left({ }^{1} \mathrm{H} \mathrm{NMR}, 400 \mathrm{MHz}, \mathrm{CDCl}_{3}\right)$

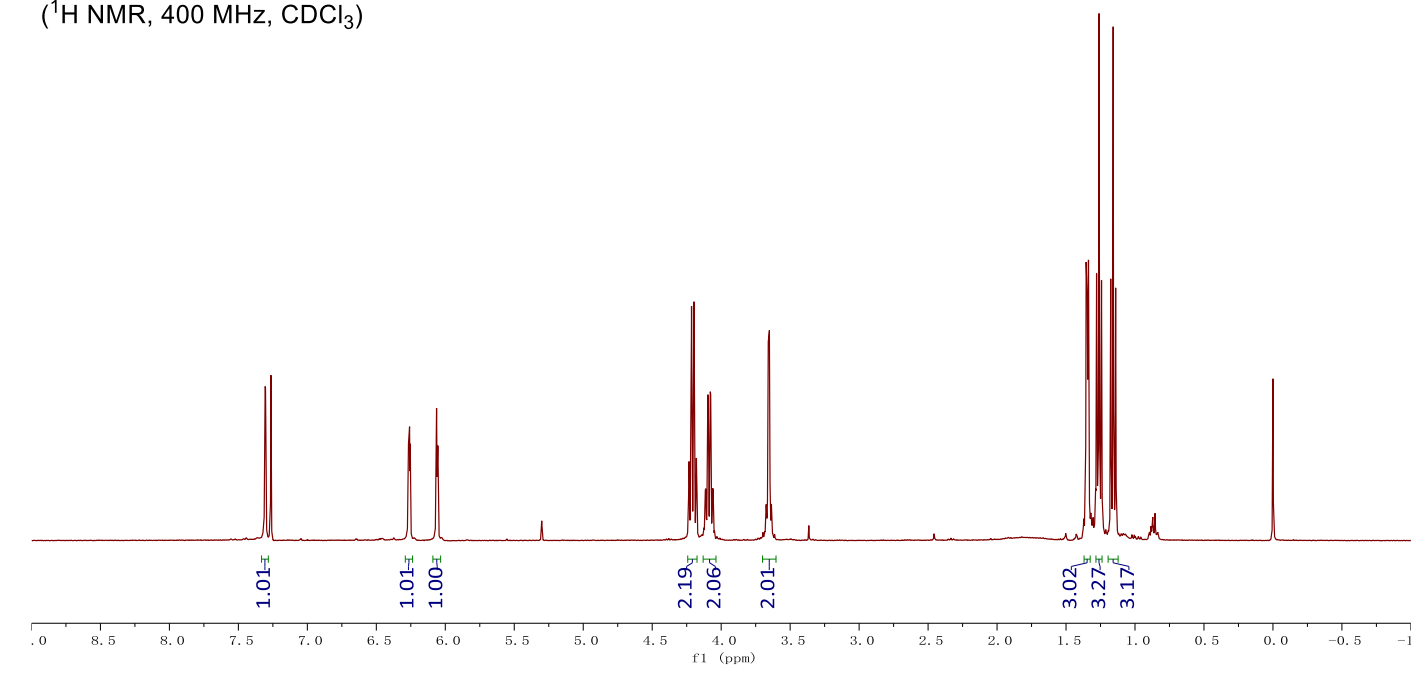

(-)-Diethyl 2-(1-cyclohexylethyl)malonate (3v)

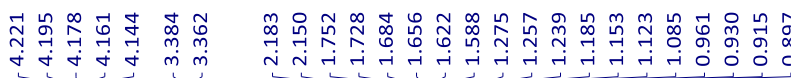

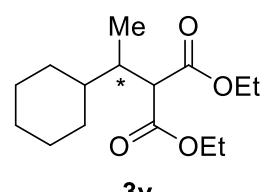

$\left({ }^{1} \mathrm{H}\right.$ NMR, $\left.400 \mathrm{MHz}, \mathrm{CDCl}_{3}\right)$

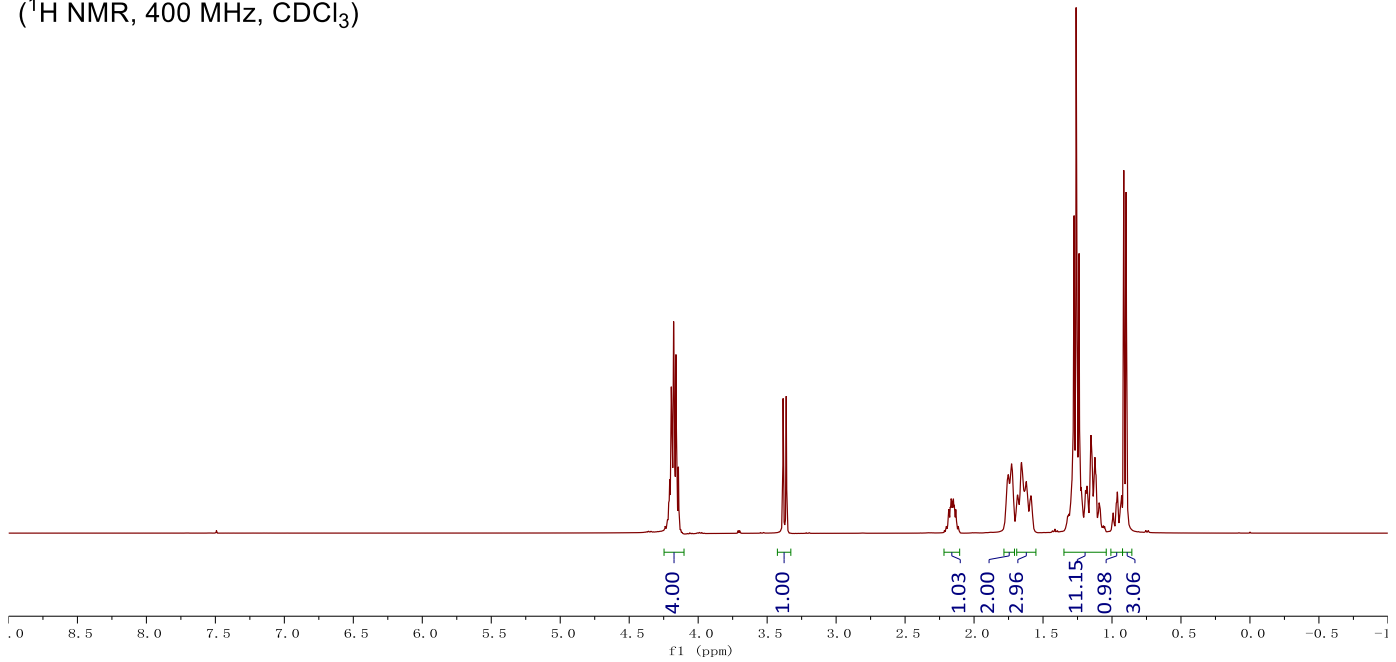


$(-)-(R)$-Ethyl-3-phenylbutanoate ((R)-4)

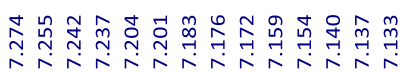

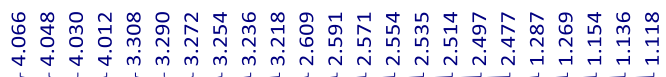

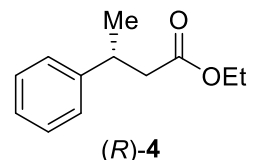

$\left({ }^{1} \mathrm{H} \mathrm{NMR}, 400 \mathrm{MHz}, \mathrm{CDCl}_{3}\right)$

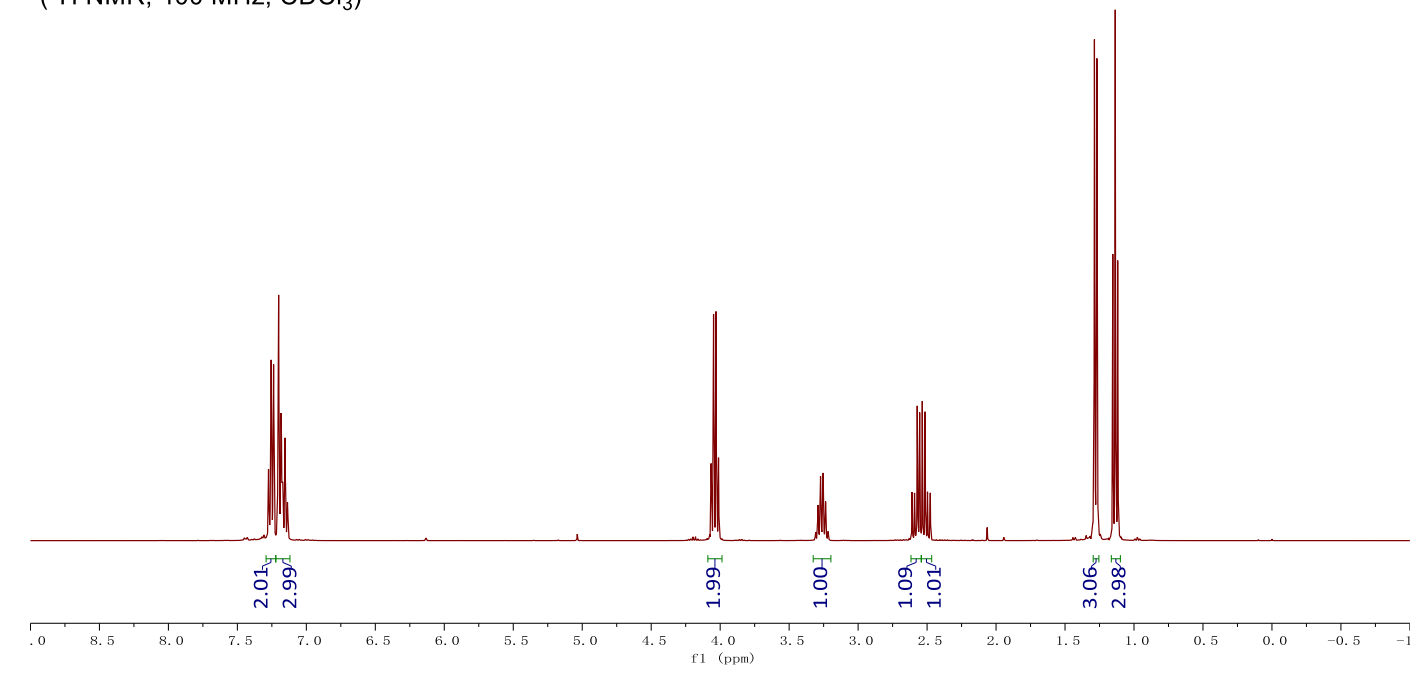

(-)-(R)-3-Phenylbutanoic acid $((R)-5)$

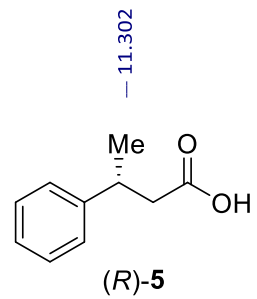

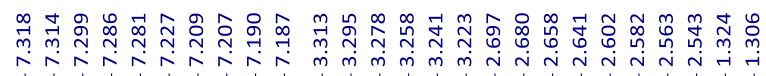

$\left({ }^{1} \mathrm{H} \mathrm{NMR}, 400 \mathrm{MHz}, \mathrm{CDCl}_{3}\right)$

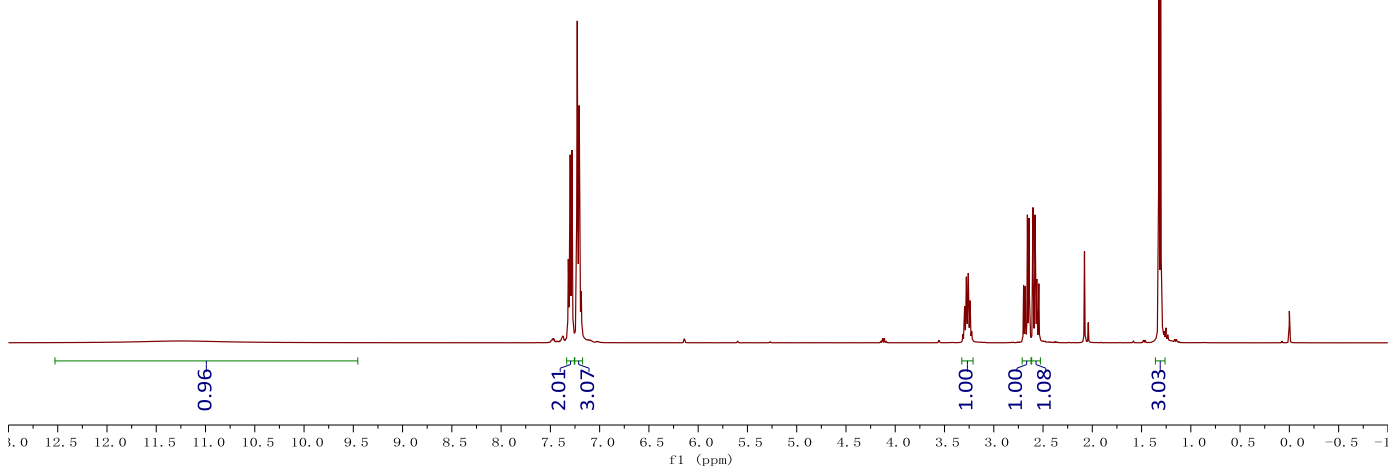


(-)-(R)-2-(ethoxycarbonyl)-3-phenylbutanoic acid (6)

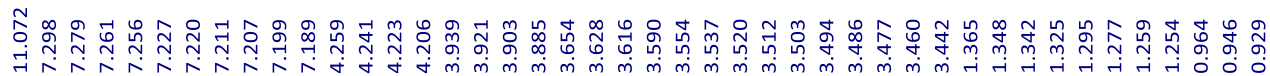

ㅅ.NNNNN

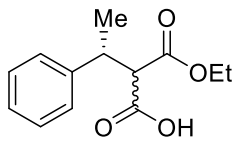

$(R)-6$

(mixture of diastereomers)

$\left({ }^{1} \mathrm{H} \mathrm{NMR}, 400 \mathrm{MHz}, \mathrm{CDCl}_{3}\right)$

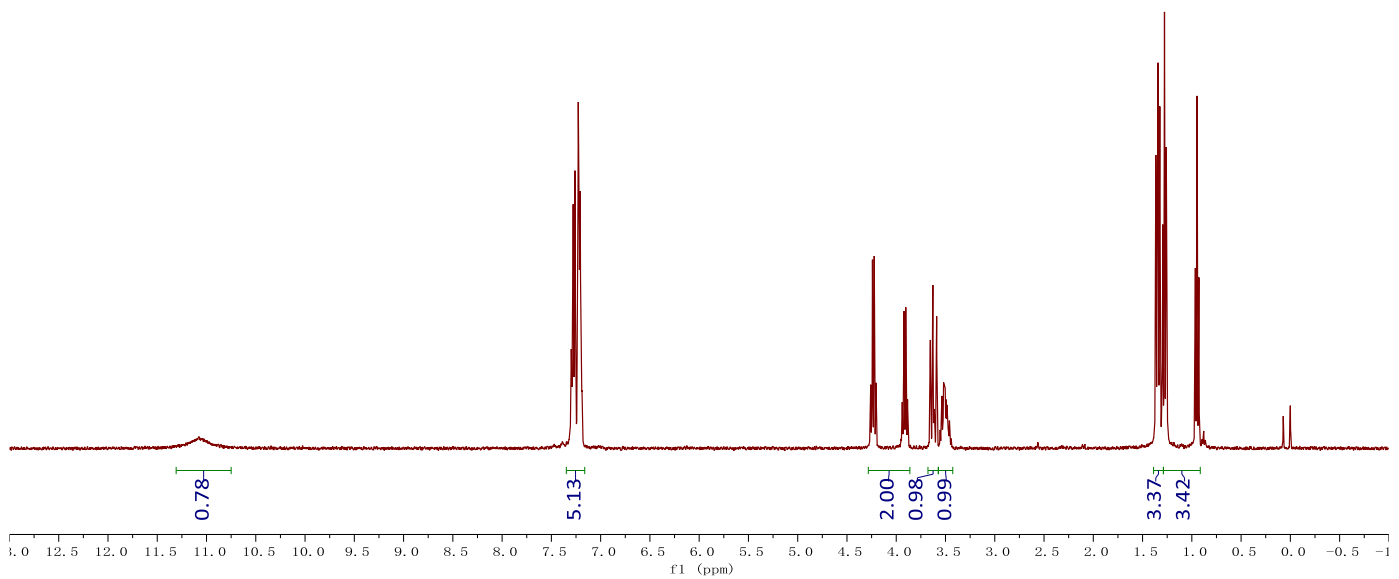

(-)-(R)-3-([1,1'-Biphenyl]-4-yl)butanoic acid $((R)-8)$

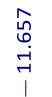

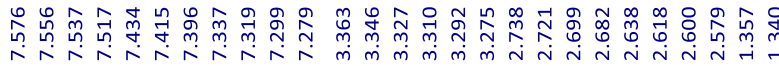

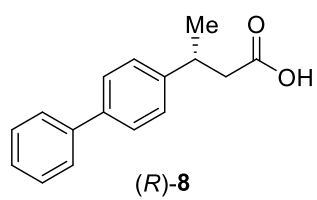

$\left({ }^{1} \mathrm{H} \mathrm{NMR}, 400 \mathrm{MHz}, \mathrm{CDCl}_{3}\right)$

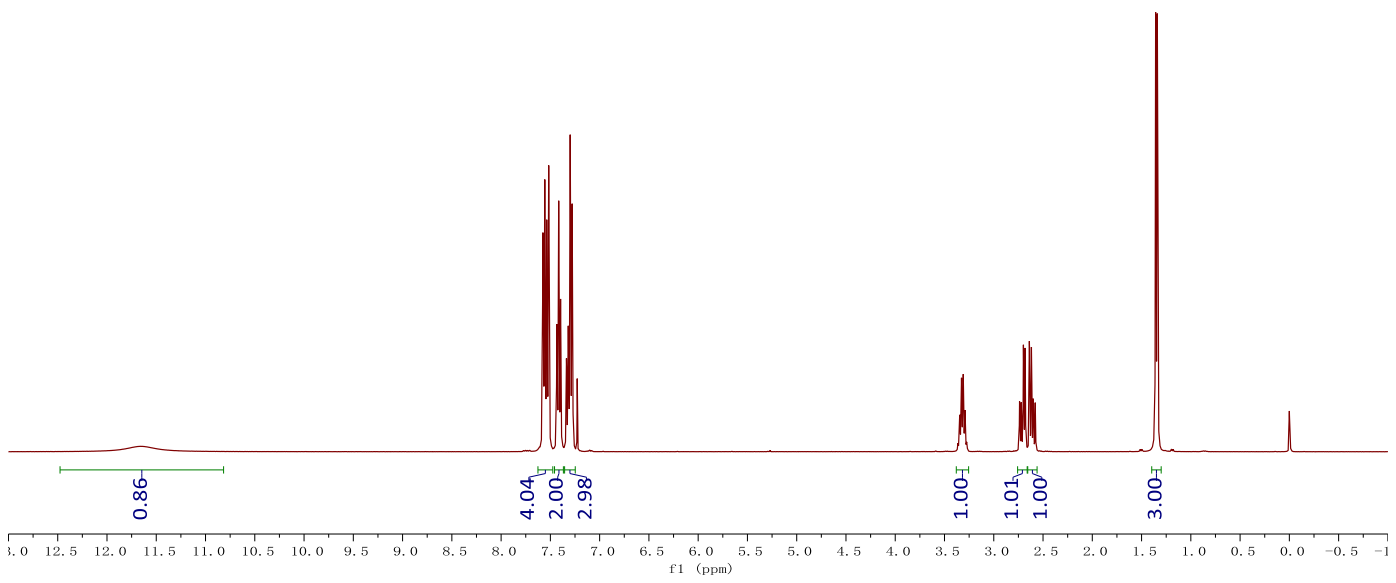


(G) HPLC Charts for Hydrogenation Products

(-)-(R)-Diethyl-2-(1-phenylethyl)malonate (3a)

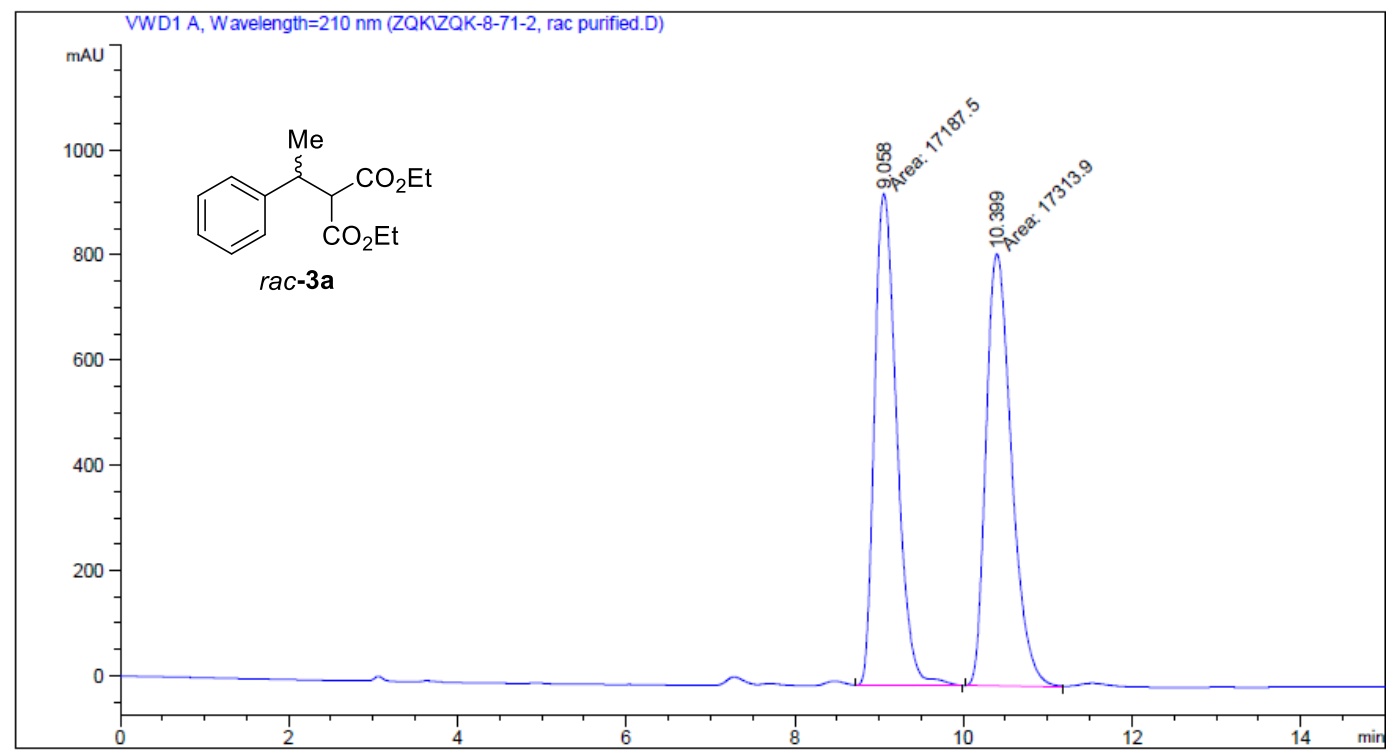

\begin{tabular}{cccccc}
$\begin{array}{c}\text { Peak RetTime Type } \\
\text { \# } \\
{[\text { min] }}\end{array}$ & $\begin{array}{c}\text { Width } \\
{[\text { min] }}\end{array}$ & $\begin{array}{c}\text { Area } \\
{[\text { mAU*s }}\end{array}$ & $\begin{array}{c}\text { Height } \\
{[\mathrm{mAU}]}\end{array}$ & $\begin{array}{c}\text { Area } \\
\%\end{array}$ \\
\hdashline-1 & $9.058 \mathrm{MM}$ & 0.3061 & $1.71731 \mathrm{e} 4$ & 934.90894 & 49.8214 \\
2 & $10.399 \mathrm{MF}$ & 0.3508 & $1.72962 \mathrm{e} 4$ & 821.68396 & 50.1786
\end{tabular}

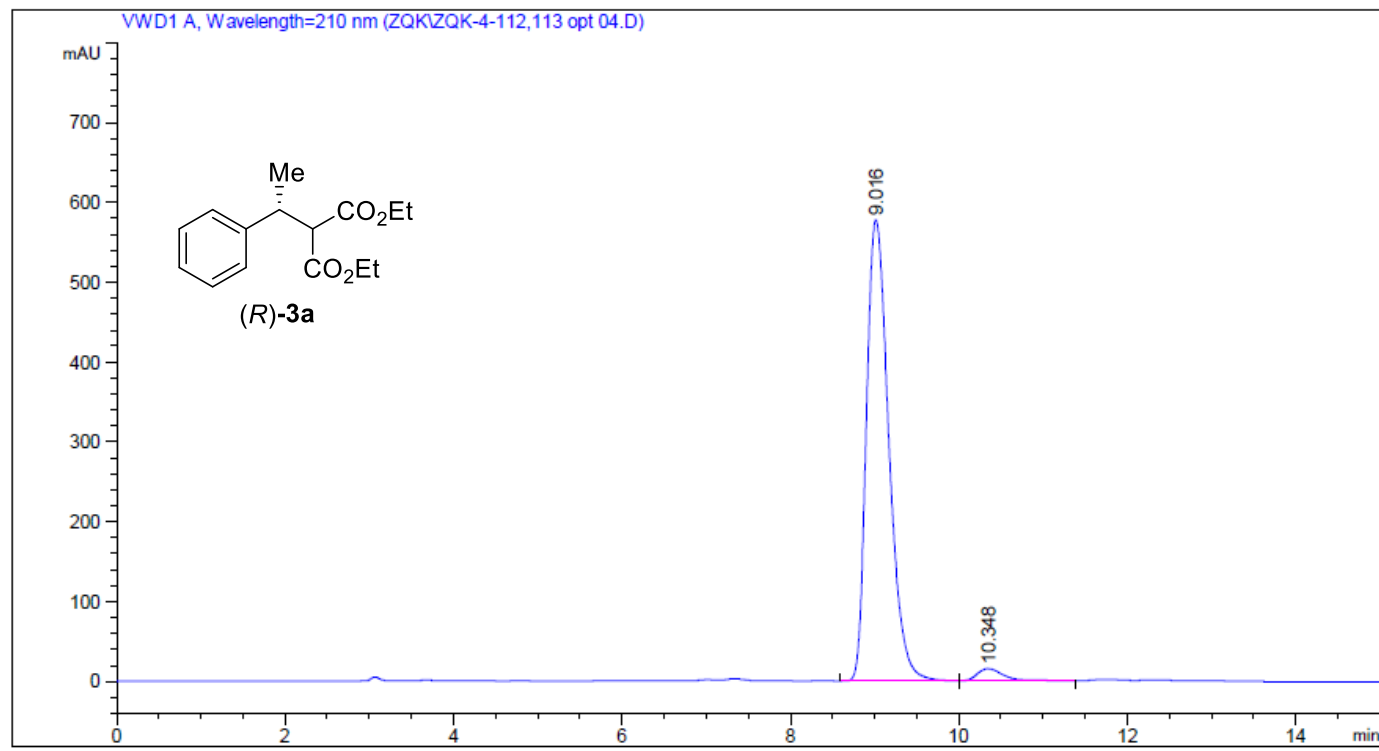
Peak RetTime Type Width Area Height Area
\# $[\mathrm{min}] \quad[\mathrm{min}] \quad[\mathrm{mAU} * \mathrm{~s}] \quad[\mathrm{mAU}] \quad \%$

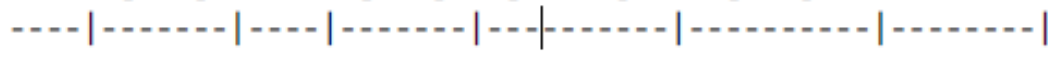
$\begin{array}{lllllll}1 & 9.016 & \text { BV } & 0.2866 & 1.06217 \mathrm{e} 4 & 577.53424 & 96.9857\end{array}$
$\begin{array}{llllll}2 & 10.348 \text { VB } & 0.3365 & 330.12592 & 15.13348 & 3.0143\end{array}$ 
(-)-Diethyl-2-(1-phenylpropyl)malonate (3b)
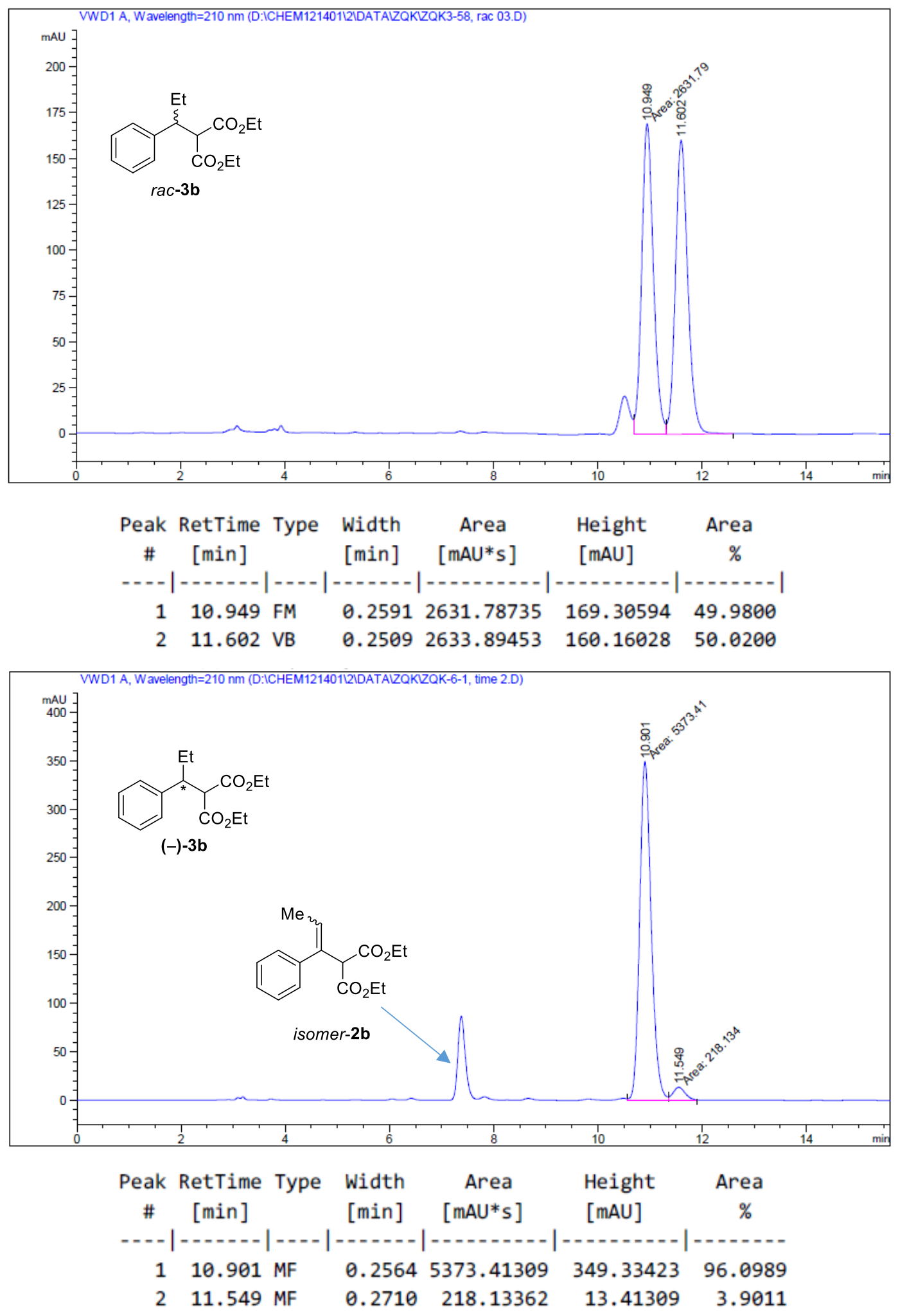
(-)-Diethyl-2-(1-phenylbutyl)malonate (3c)
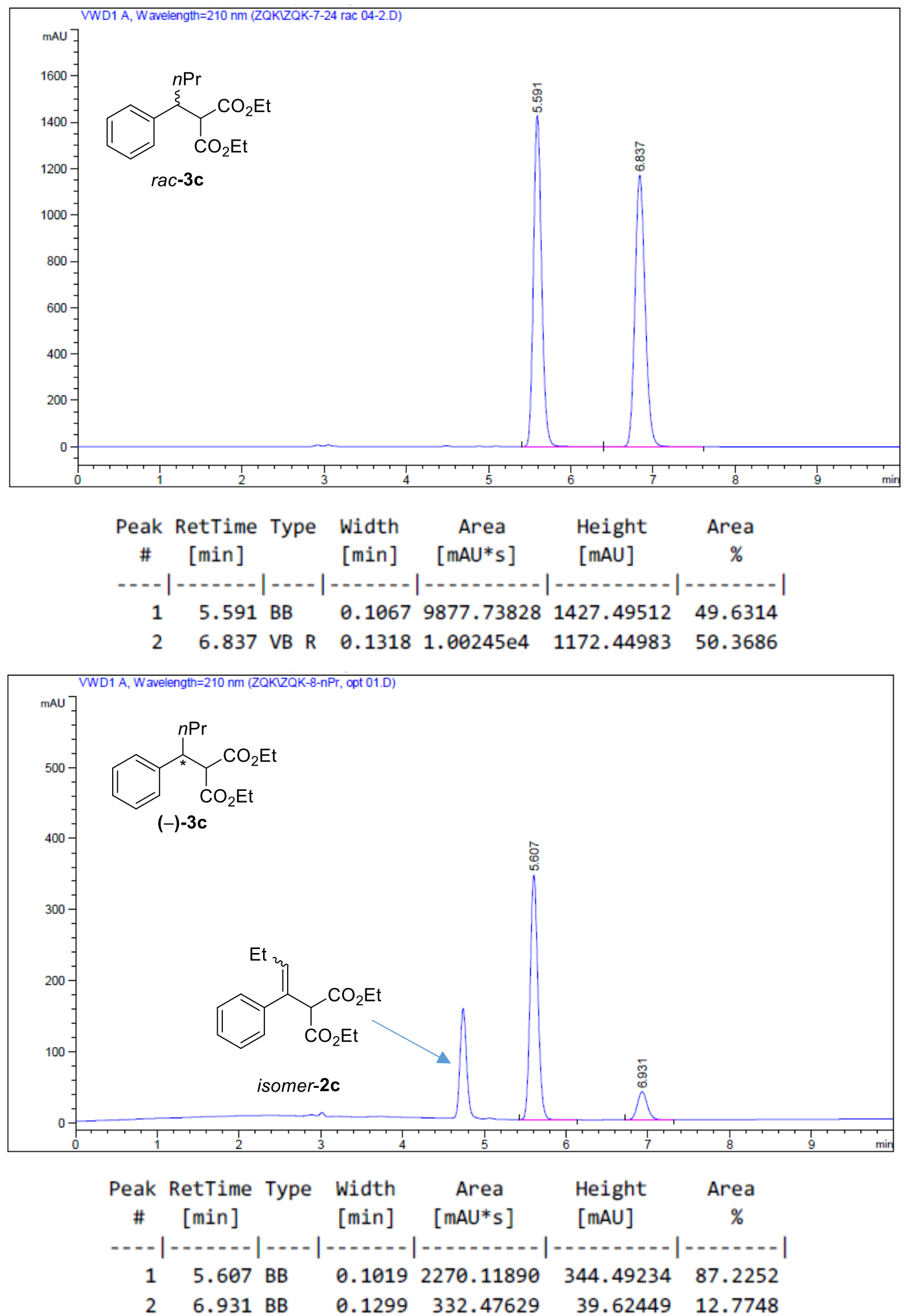
(-)-(R)-Diethyl-2-(1-(4-fluorophenyl)ethyl)malonate (3d)
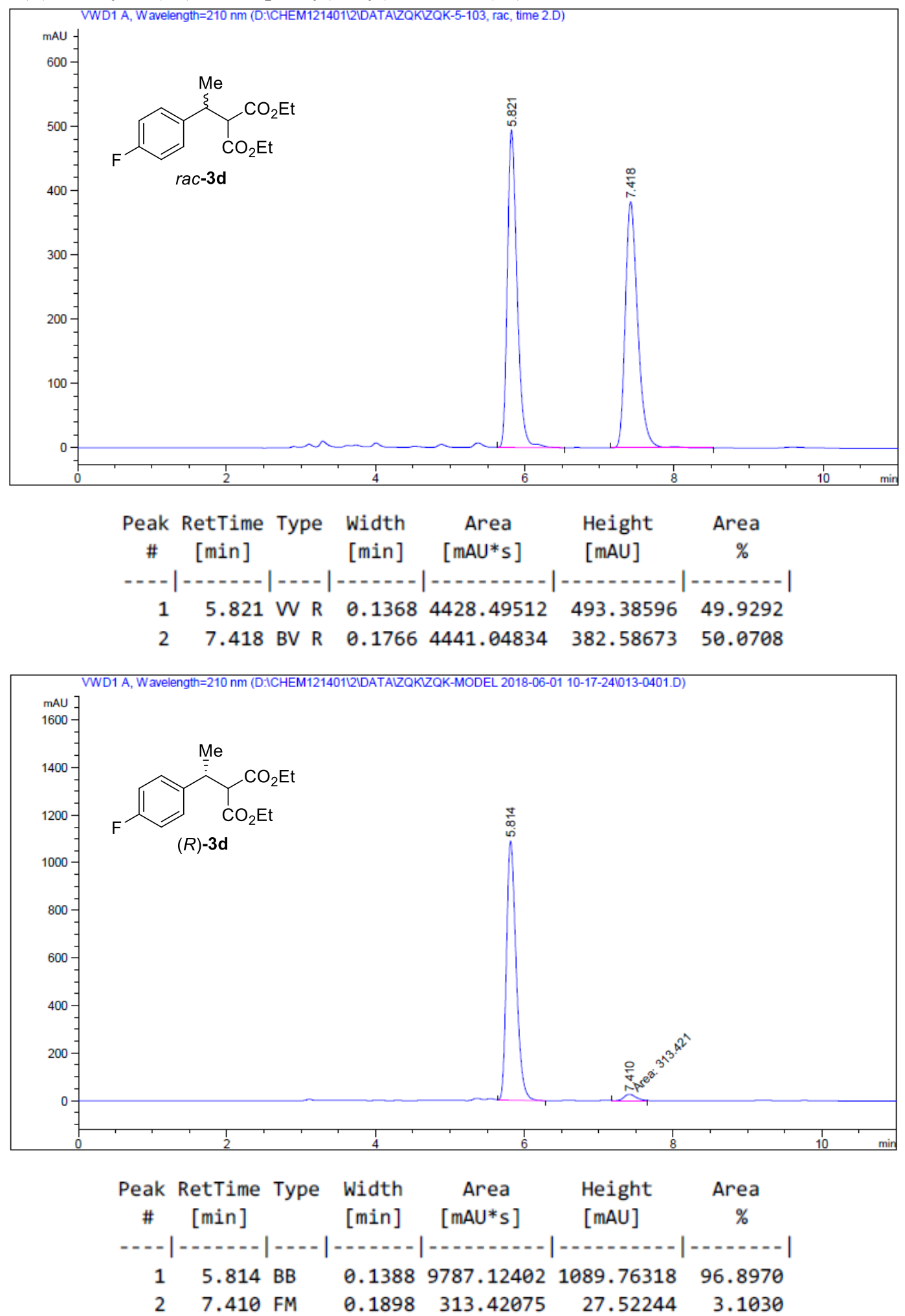
(-)-Diethyl-2-(1-(4-chlorophenyl)ethyl)malonate (3e)
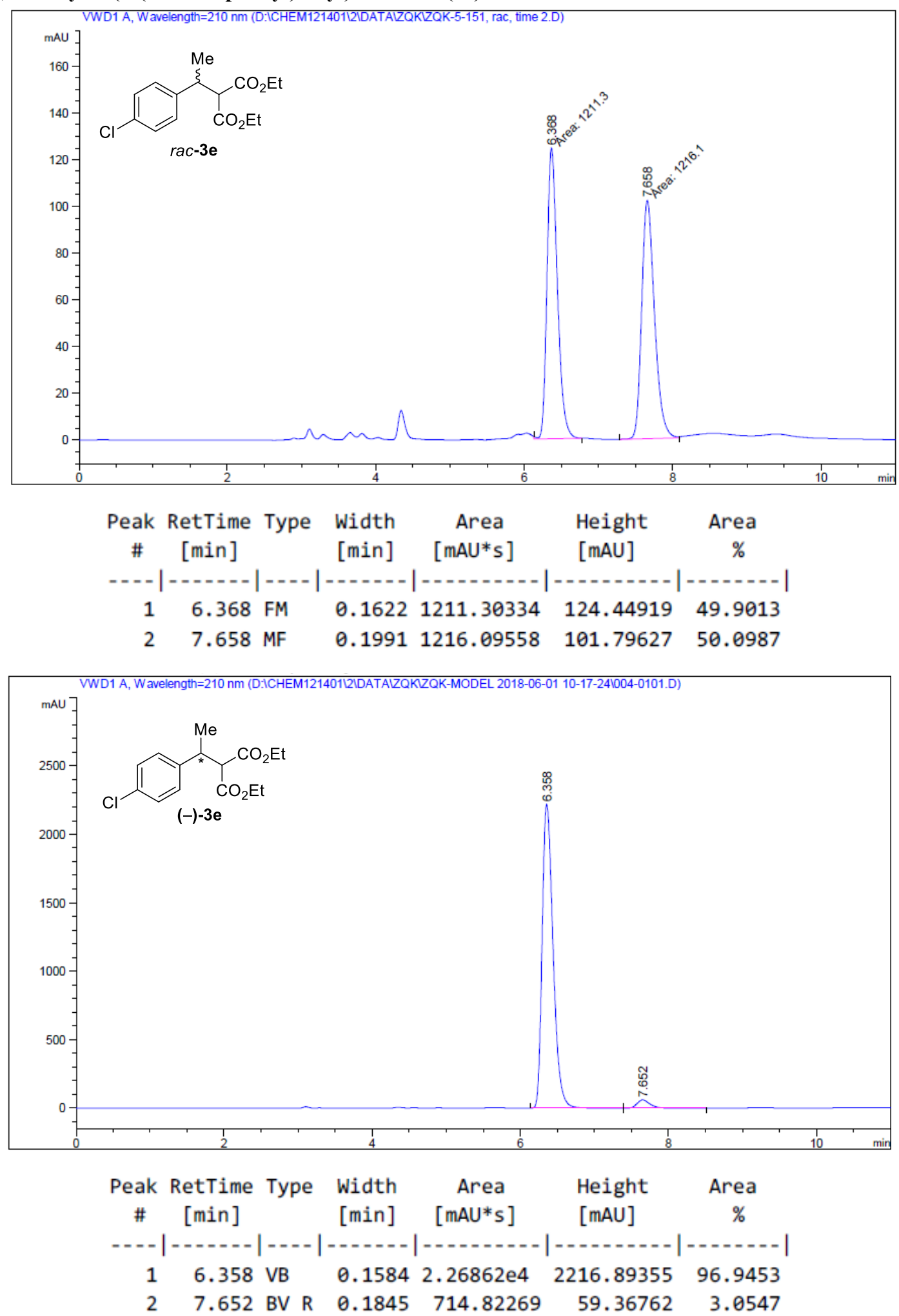
(-)-Diethyl-2-(1-(4-bromophenyl)ethyl)malonate (3f)
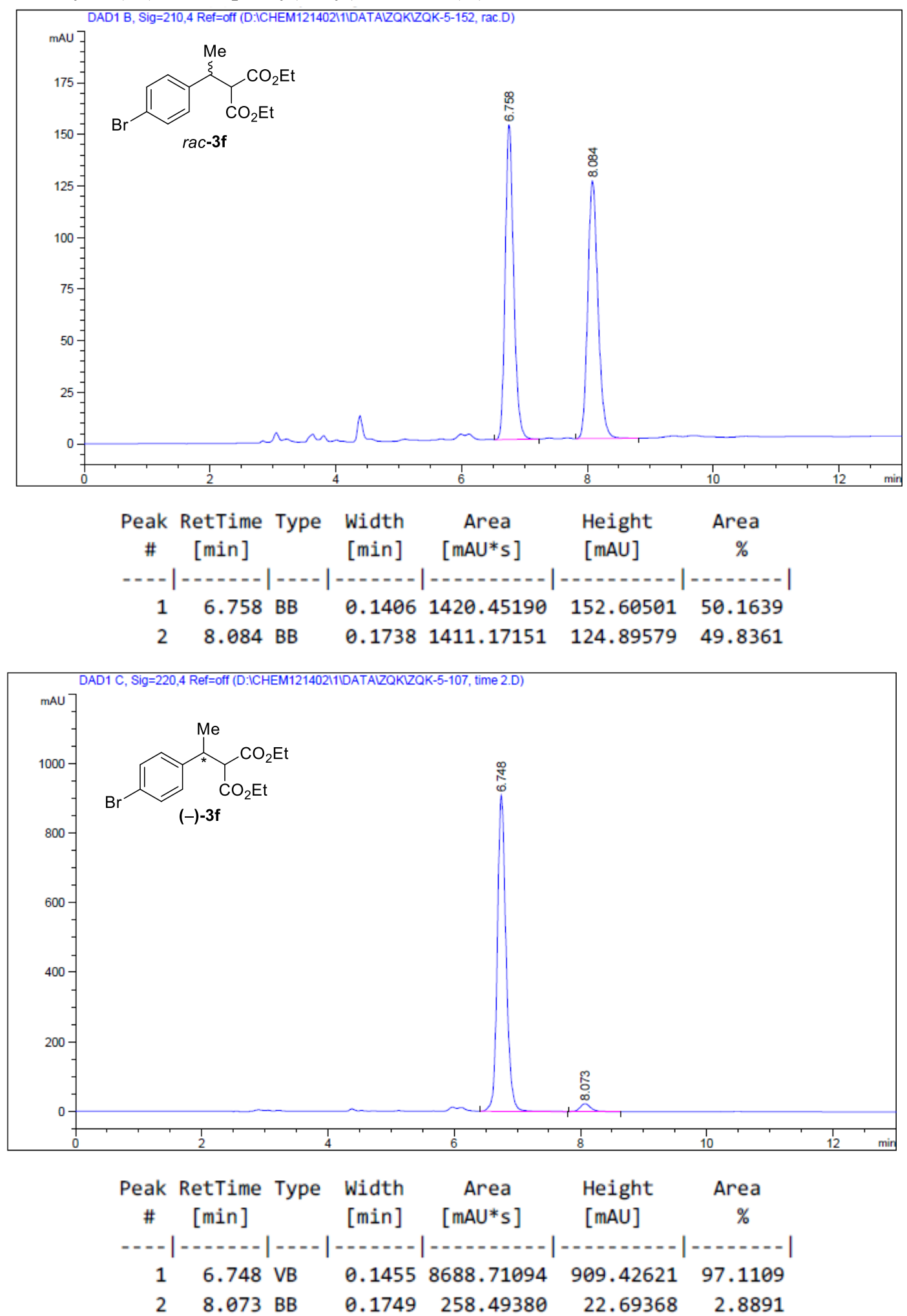
(-)-(R)-Diethyl-2-(1-(4-(trifluoromethyl)phenyl)ethyl)malonate (3g)

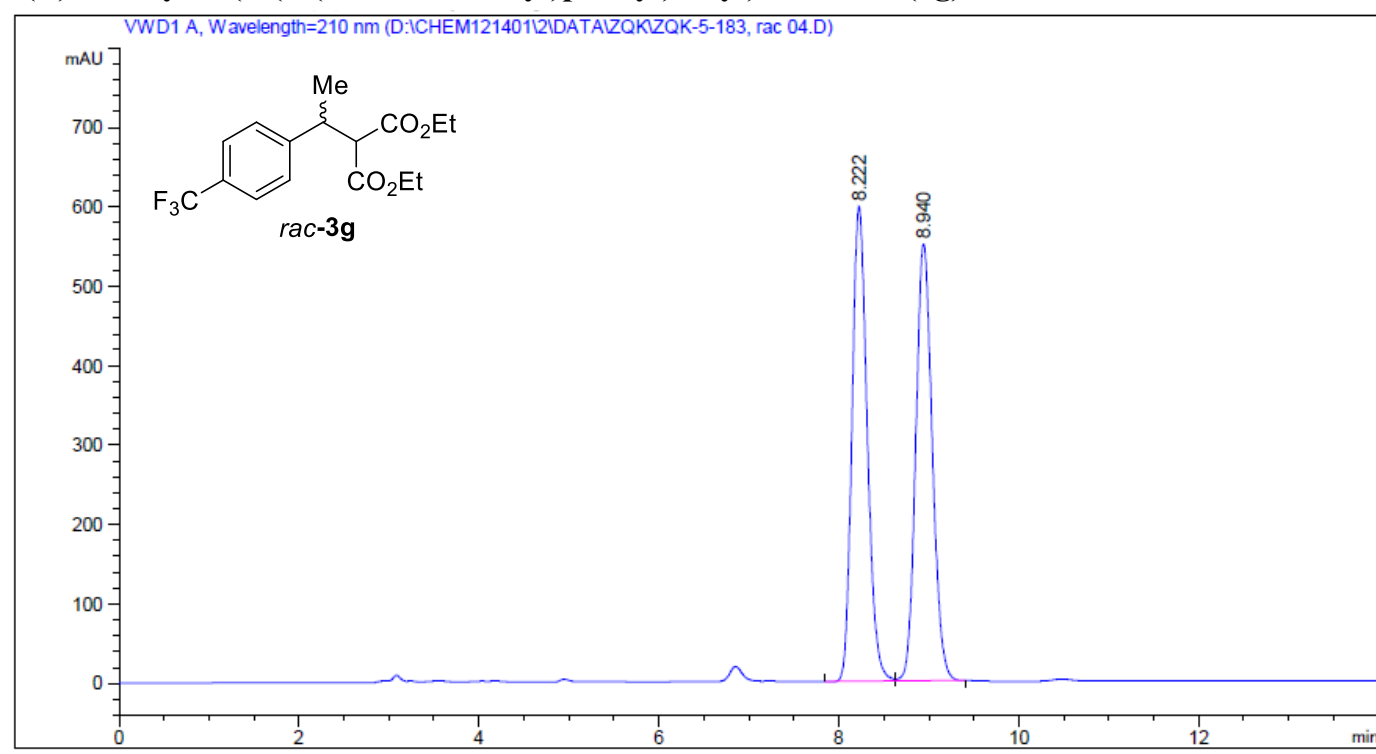
Peak RetTime Type Width Area Height Area

$\begin{array}{lllll}\# & {[\mathrm{~min}]} & {[\mathrm{min}]} & {[\mathrm{mAU} * \mathrm{~s}]} & {[\mathrm{mAU}]}\end{array}$

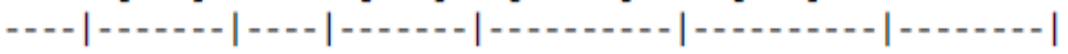
$\begin{array}{lllllll}1 & 8.222 & \text { BV } & 0.1820 & 7025.03223 & 598.33868 & 50.0333 \\ 2 & 8.940 & \text { VB } & 0.1967 & 7015.69385 & 550.46454 & 49.9667\end{array}$

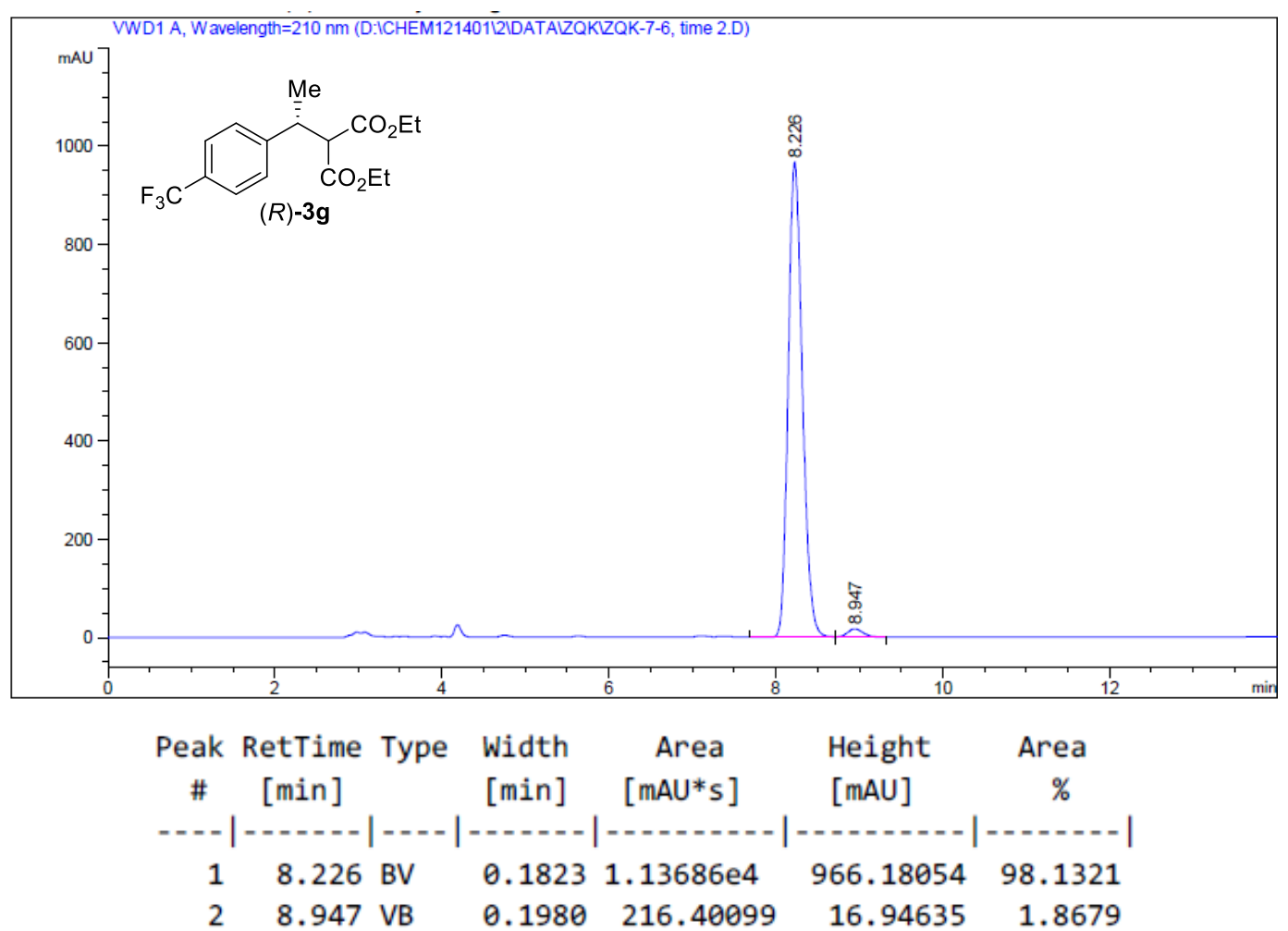


(-)-Diethyl-2-(1-(4-(ethoxycarbonyl)phenyl)ethyl)malonate (3h)
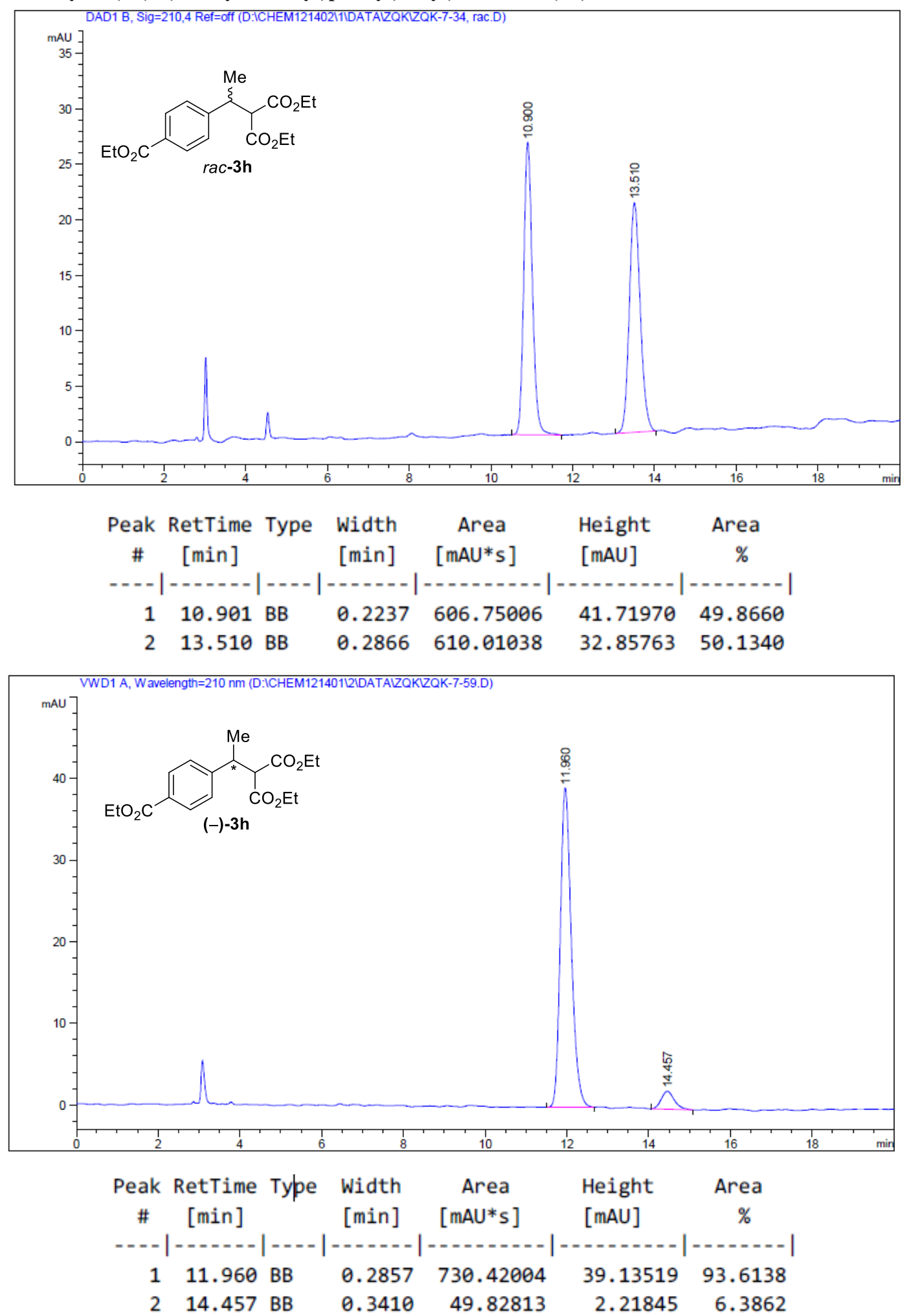
(-)-(R)-Diethyl-2-(1-([1,1'-biphenyl]-4-yl)ethyl)malonate (3i)
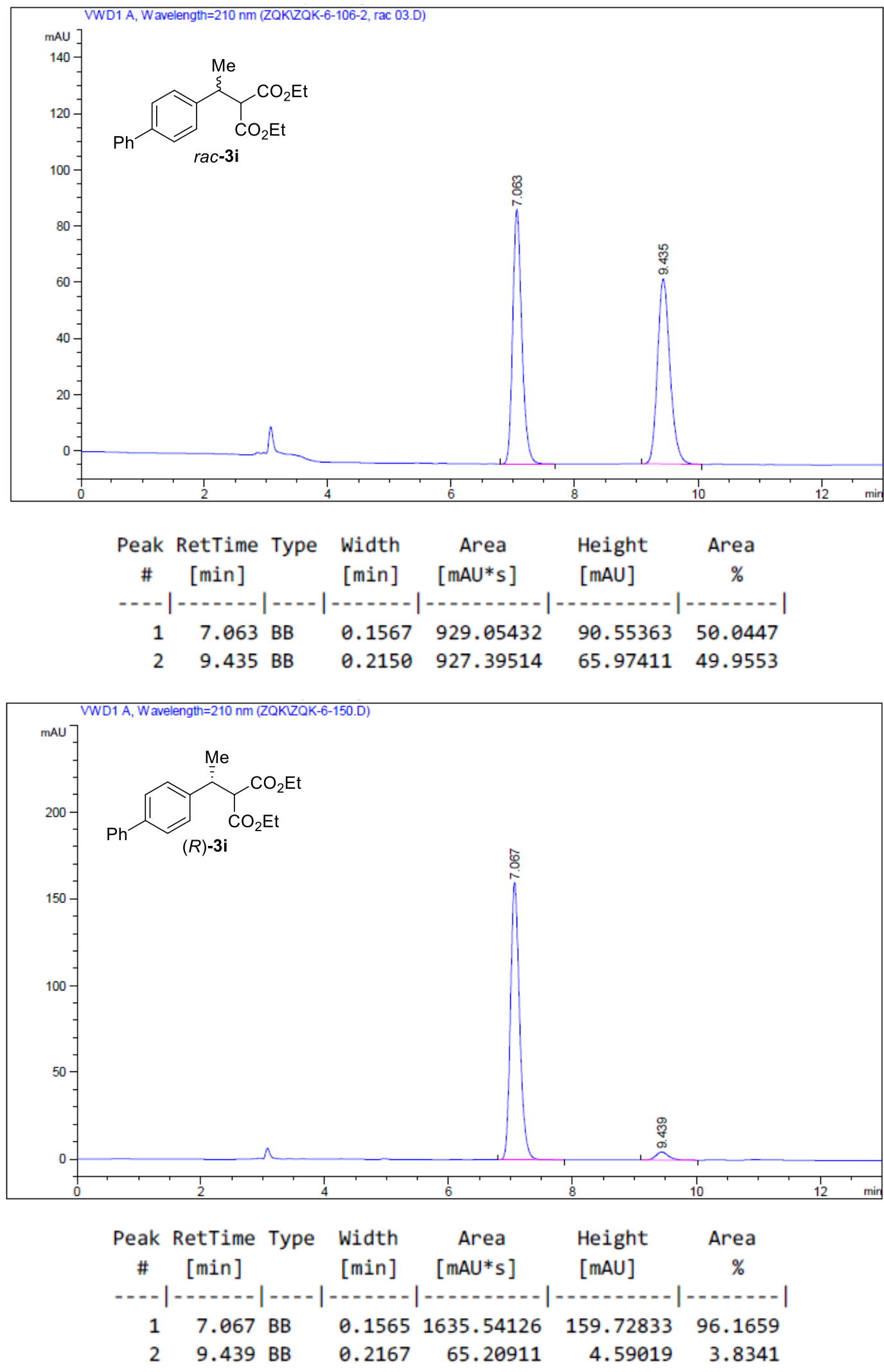
(-)-Diethyl-2-(1-(p-tolyl)ethyl)malonate (3j)
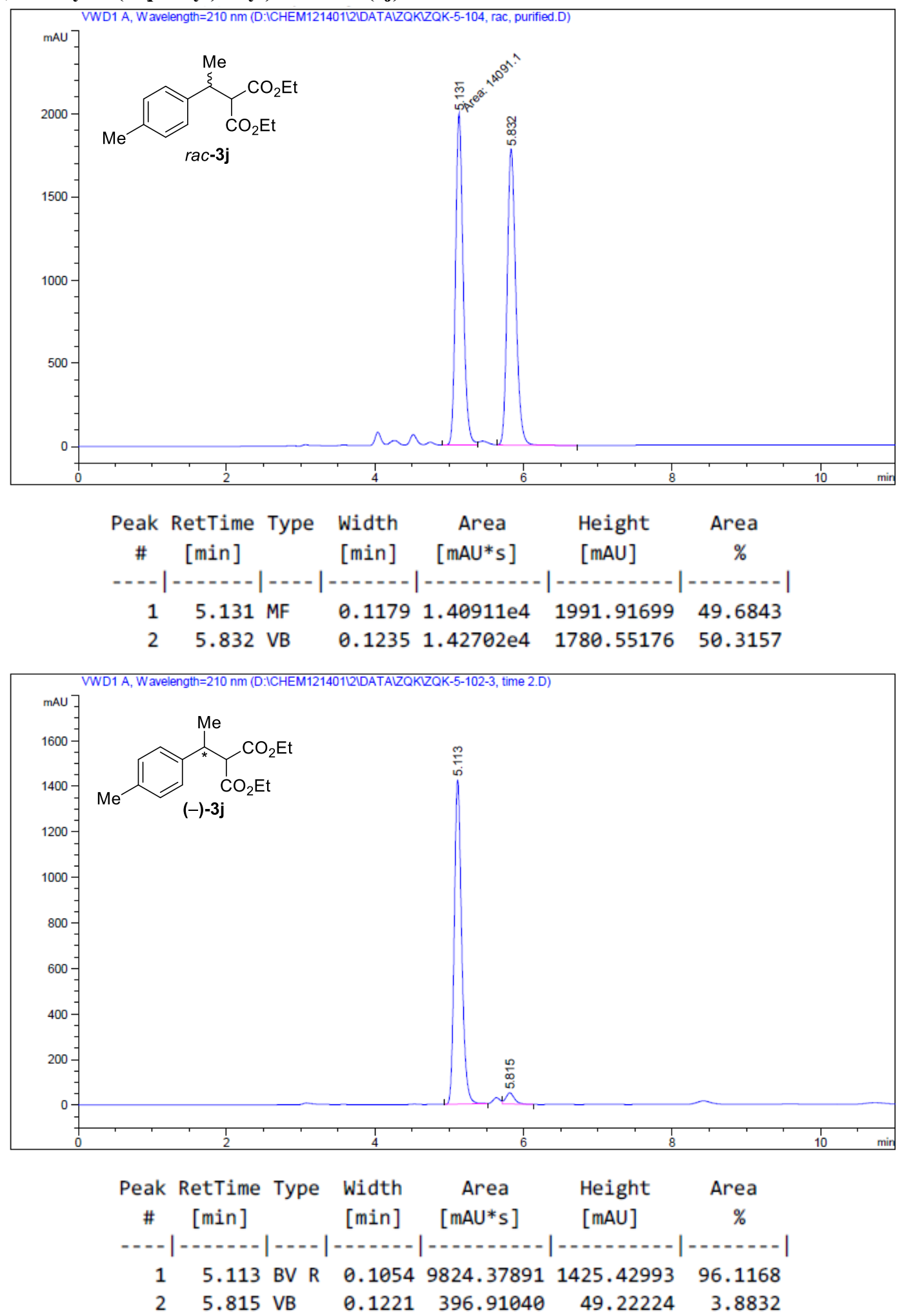
(-)-(R)-Diethyl-2-(1-(4-methoxyphenyl)ethyl)malonate (3k)
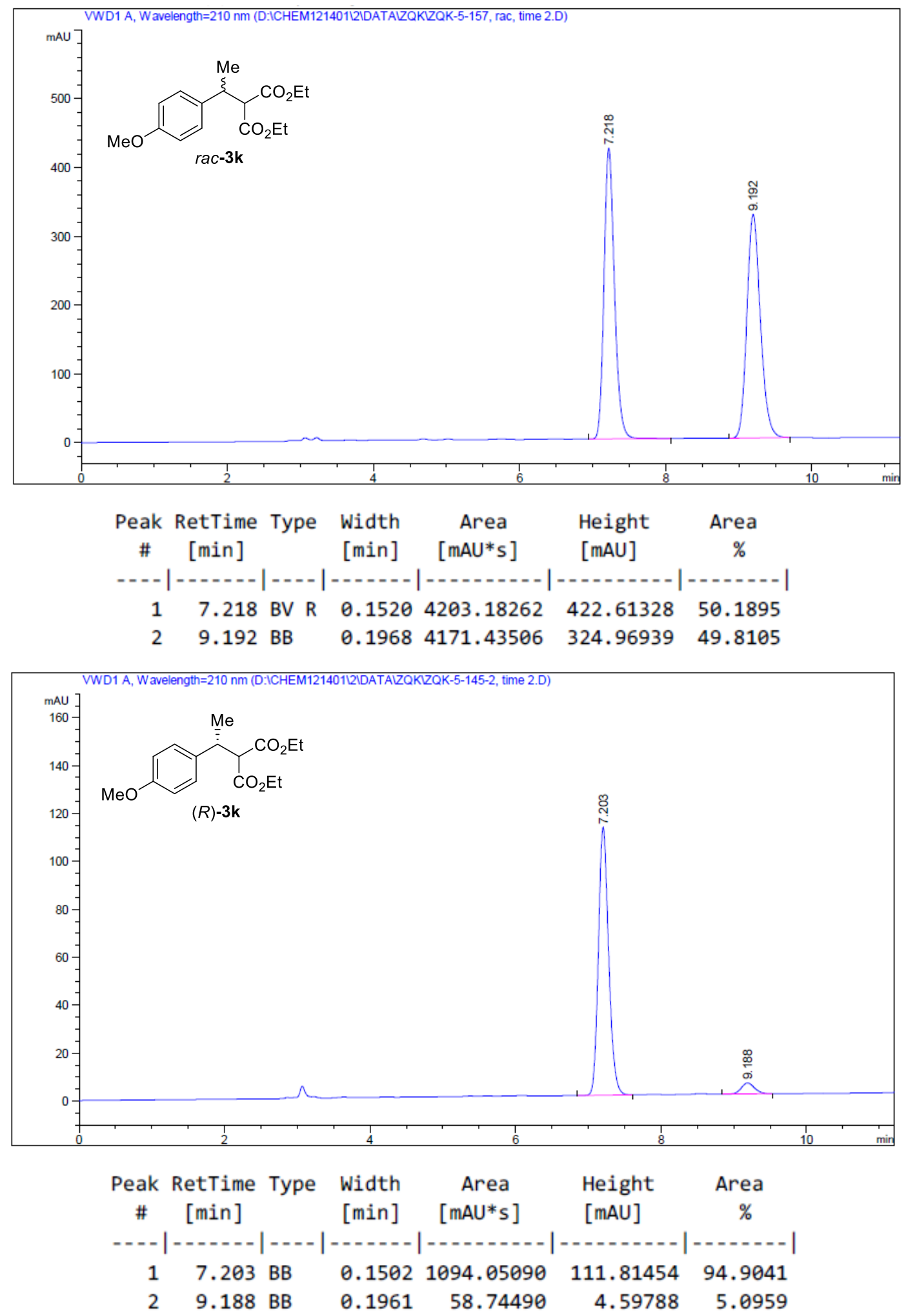
(-)-Diethyl-2-(1-(3-fluorophenyl)ethyl)malonate (3l)
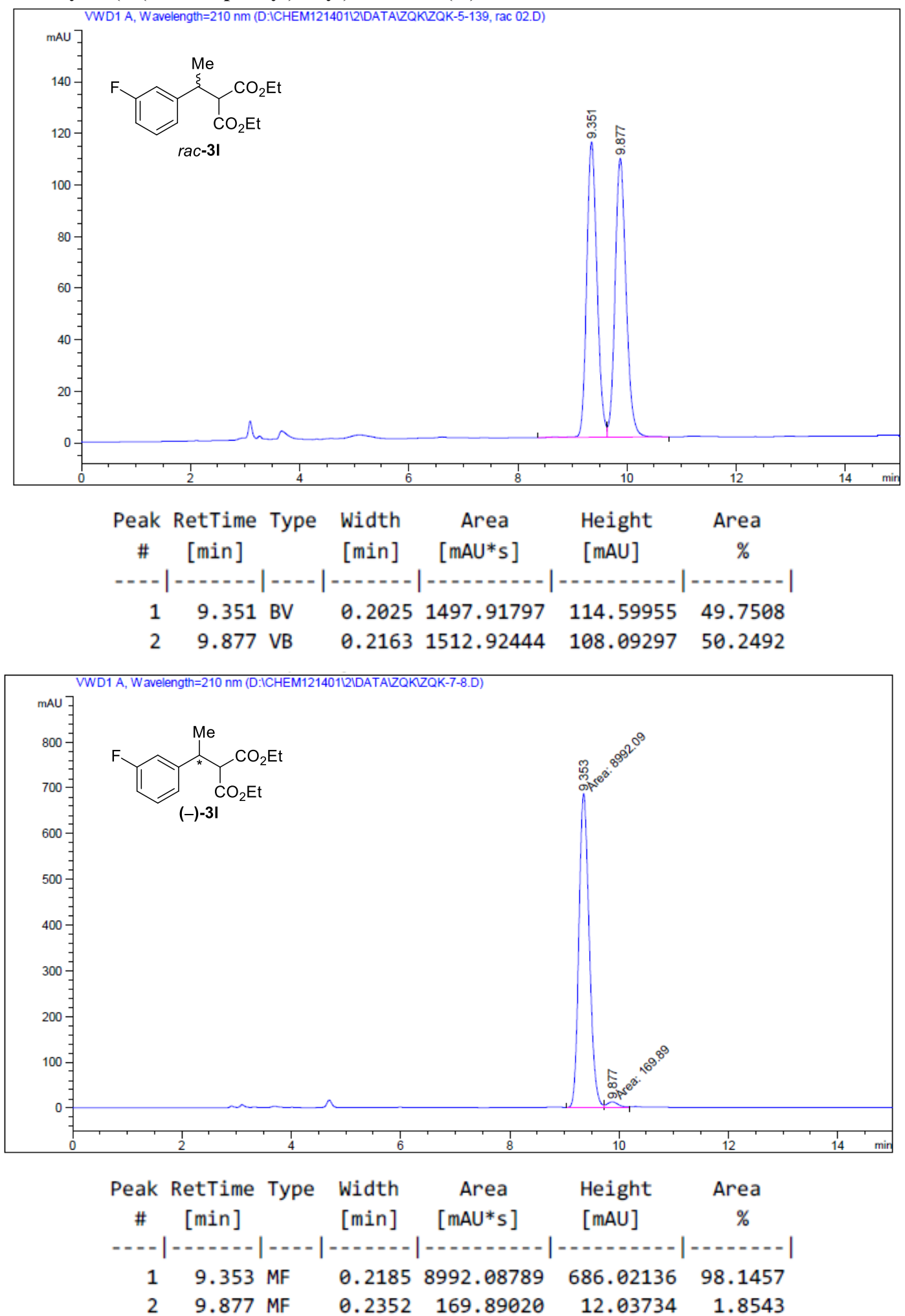
(-)-Diethyl-2-(1-(m-tolyl)ethyl)malonate (3m)
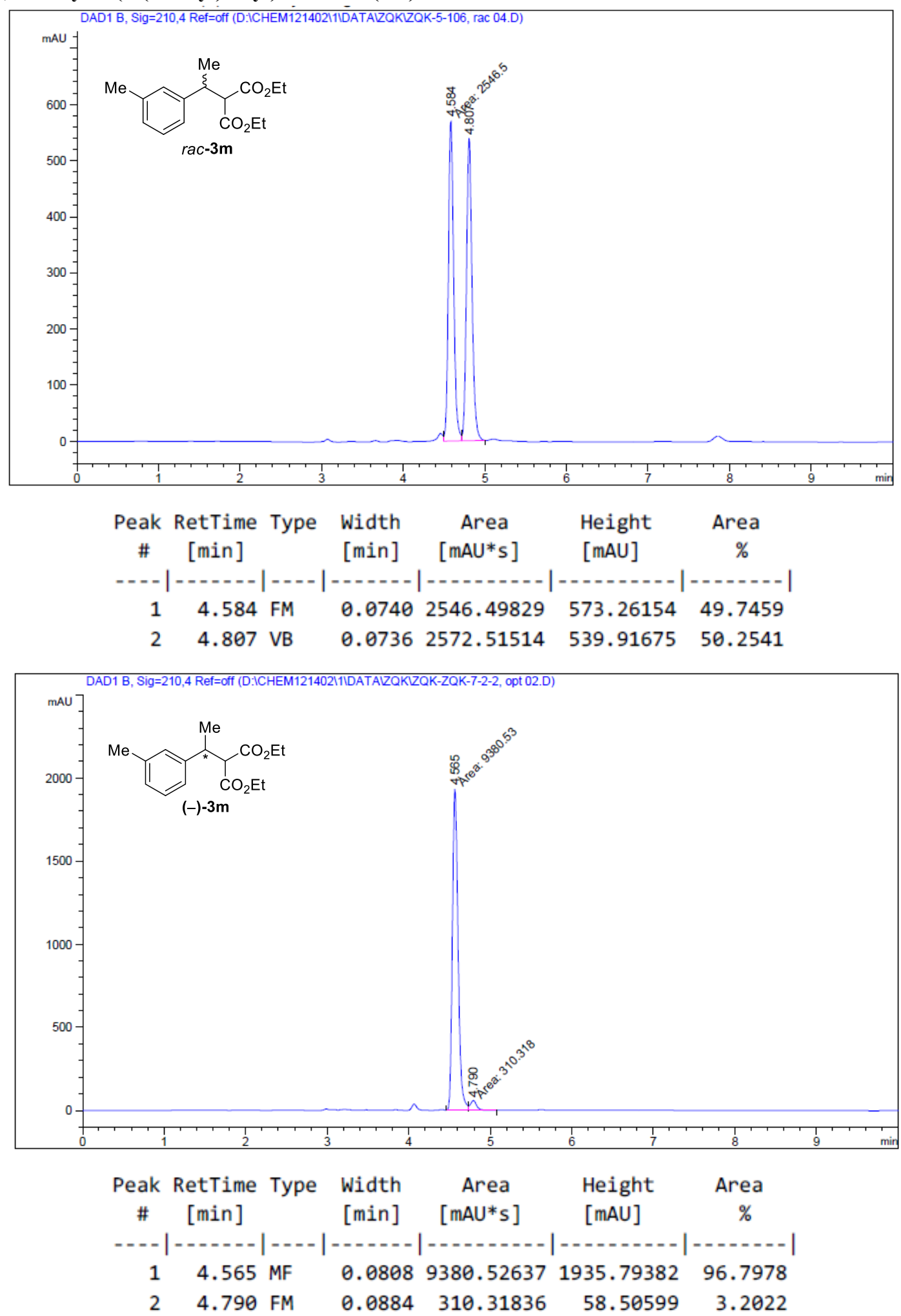
(-)-Diethyl-2-(1-(3-methoxyphenyl)ethyl)malonate (3n)
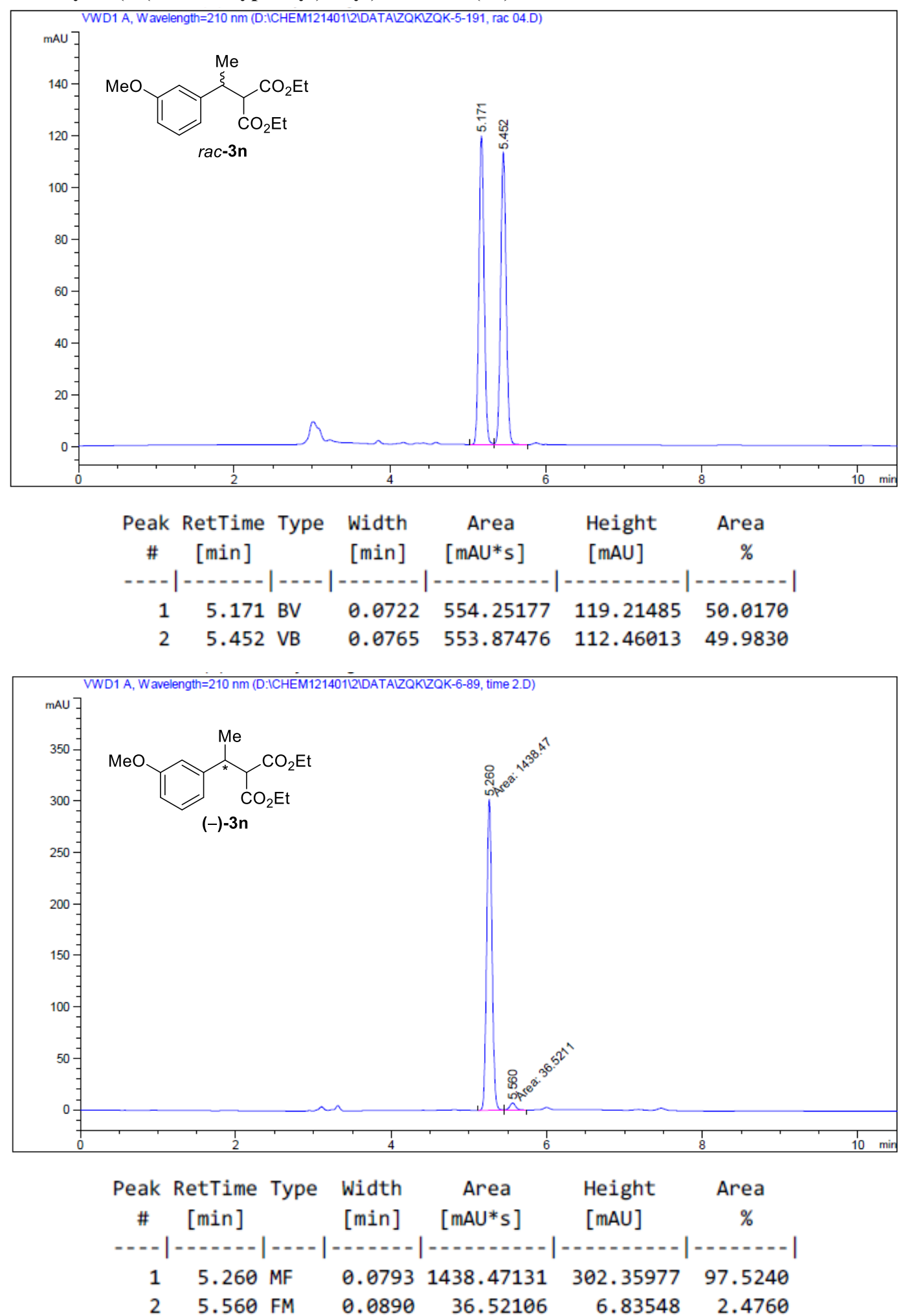
(-)-Diethyl-2-(1-(2-fluorophenyl)ethyl)malonate (3o)
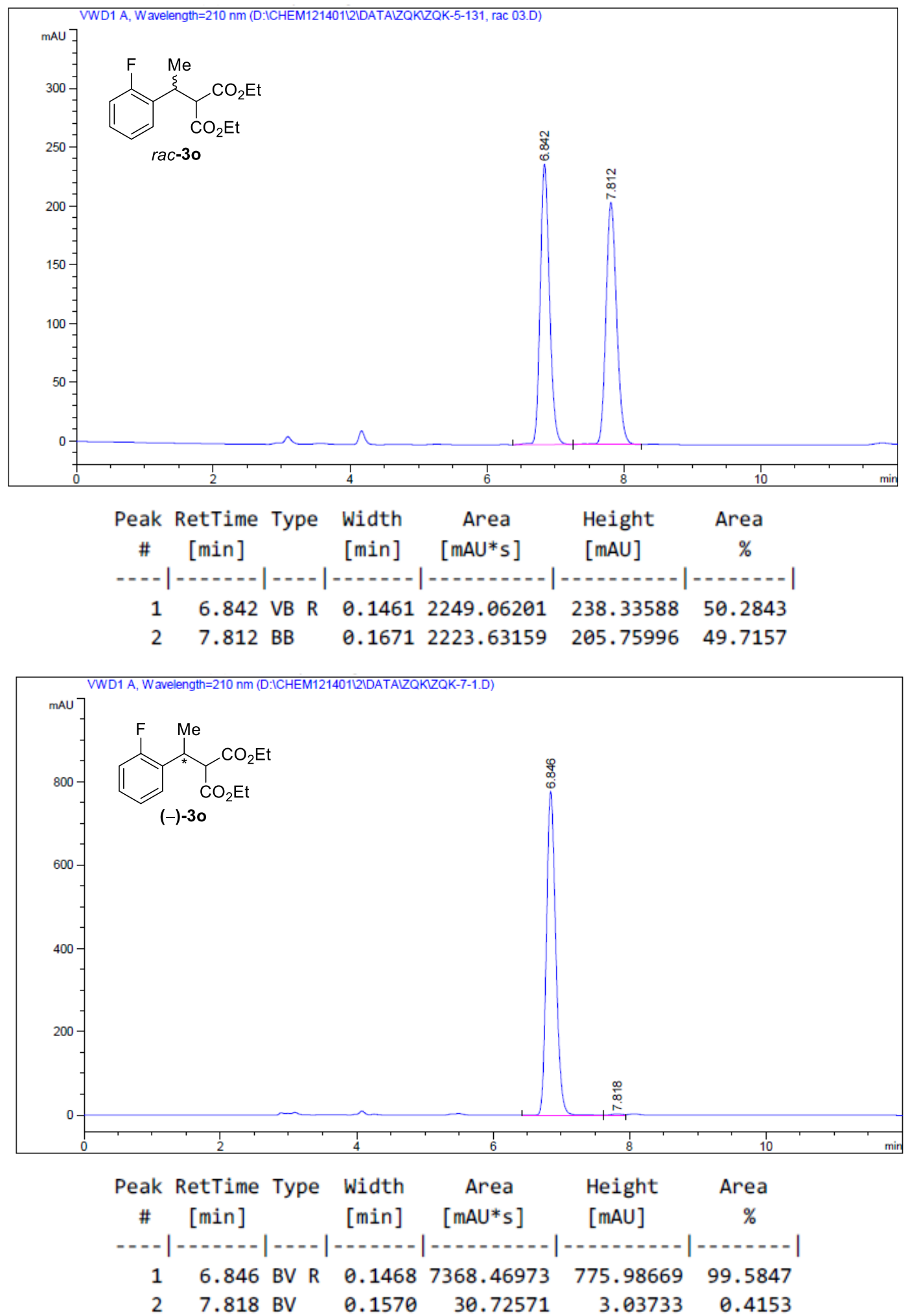
(-)-Diethyl-2-(1-(o-tolyl)ethyl)malonate (3p)
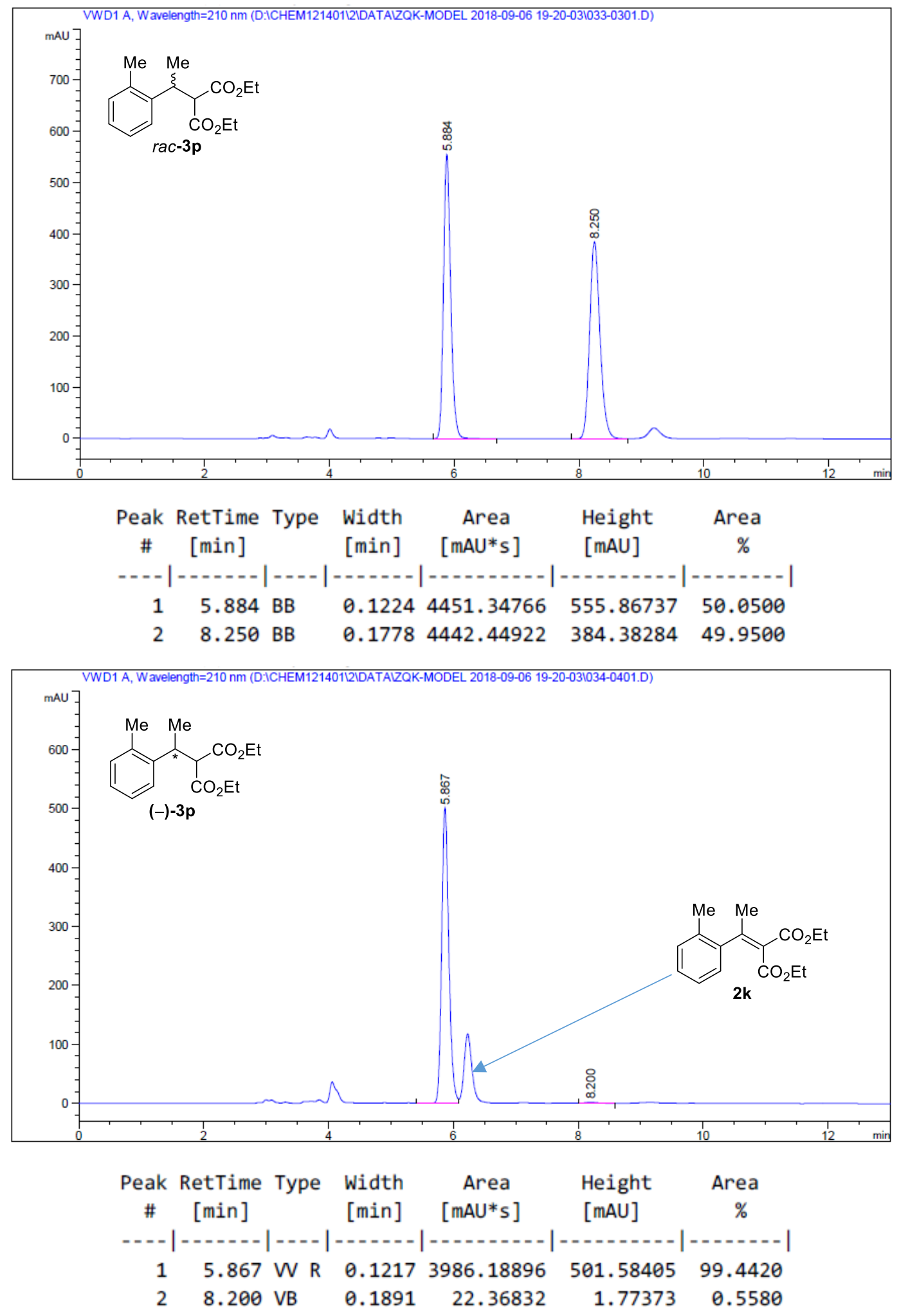
(-)-(R)-Diethyl-2-(1-(benzo[d][1,3]dioxol-5-yl)ethyl)malonate (3q)
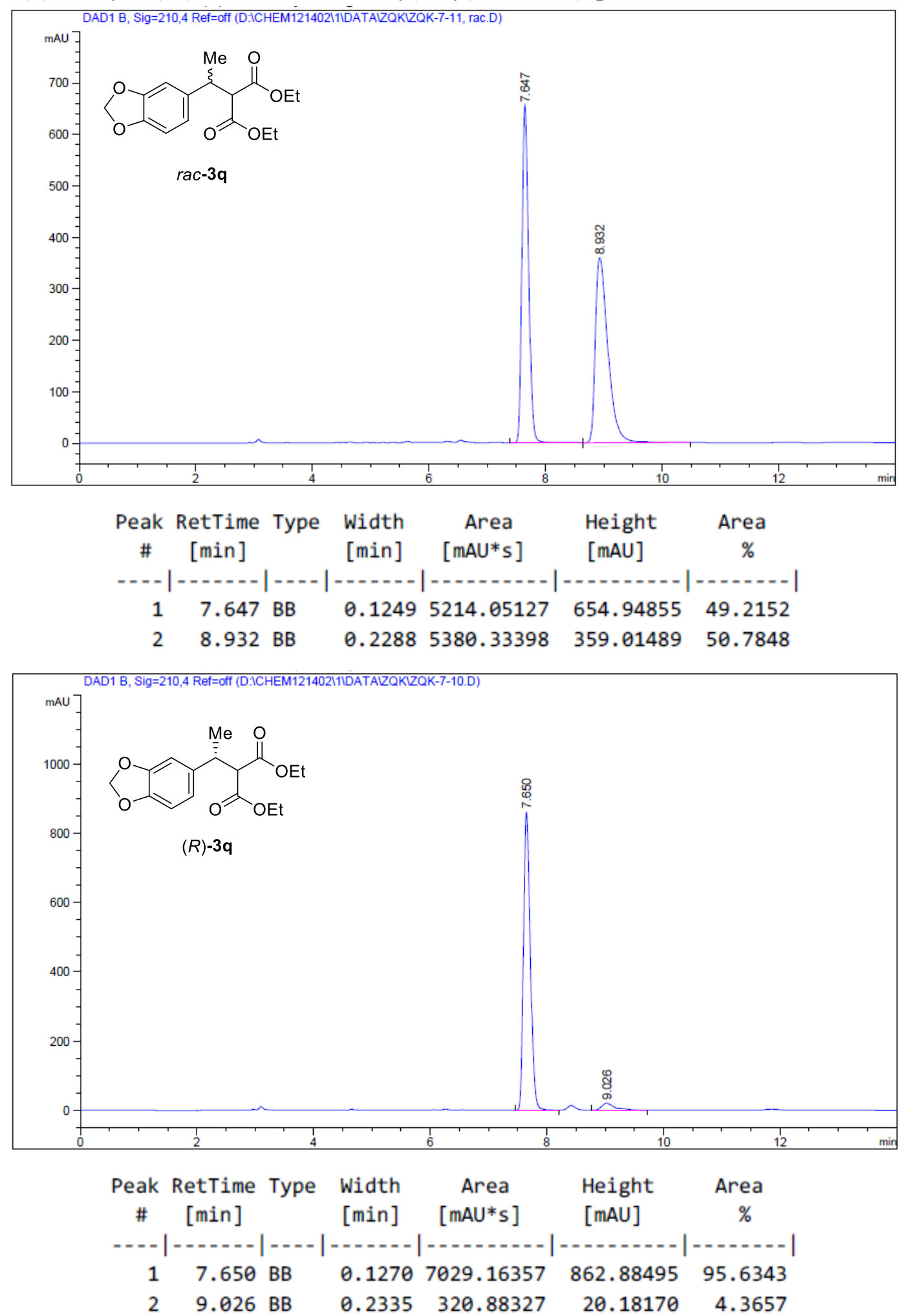
(+)-Diethyl-2-(1-(naphthalen-1-yl)ethyl)malonate (3r)
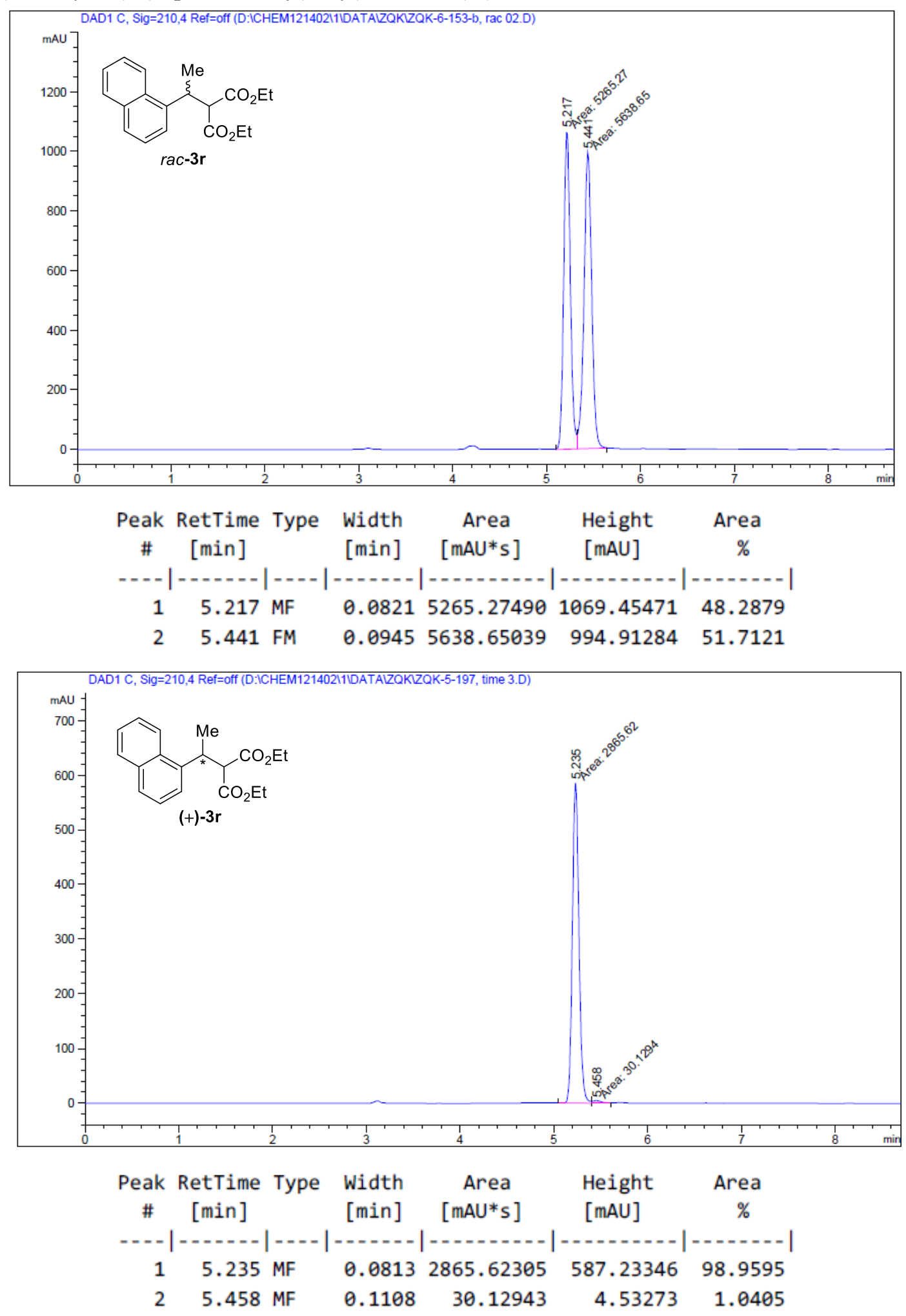
(-)-Diethyl-2-(1-(naphthalen-2-yl)ethyl)malonate (3s)
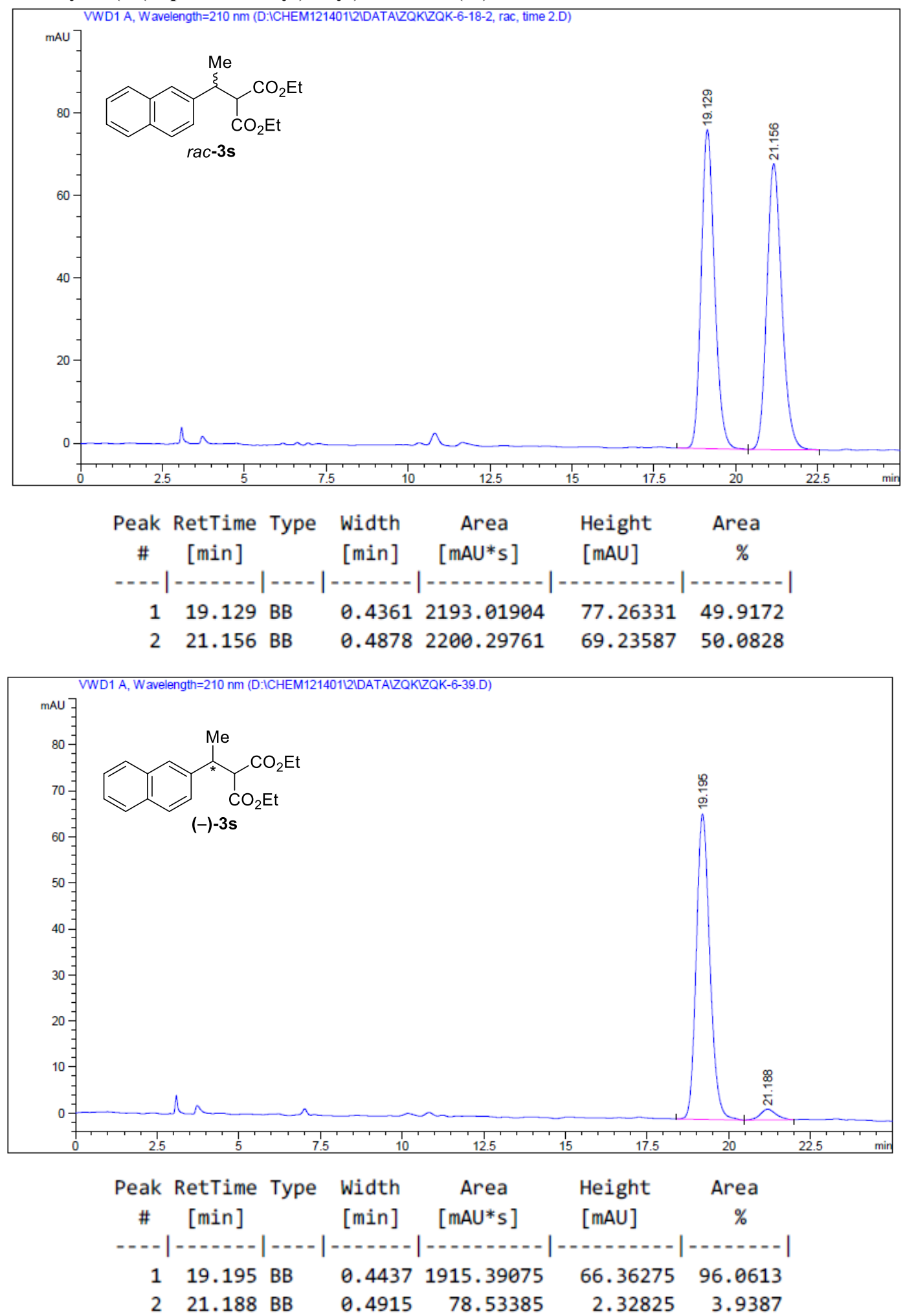
(-)-Diethyl-2-(1-(thiophen-2-yl)ethyl)malonate (3t)
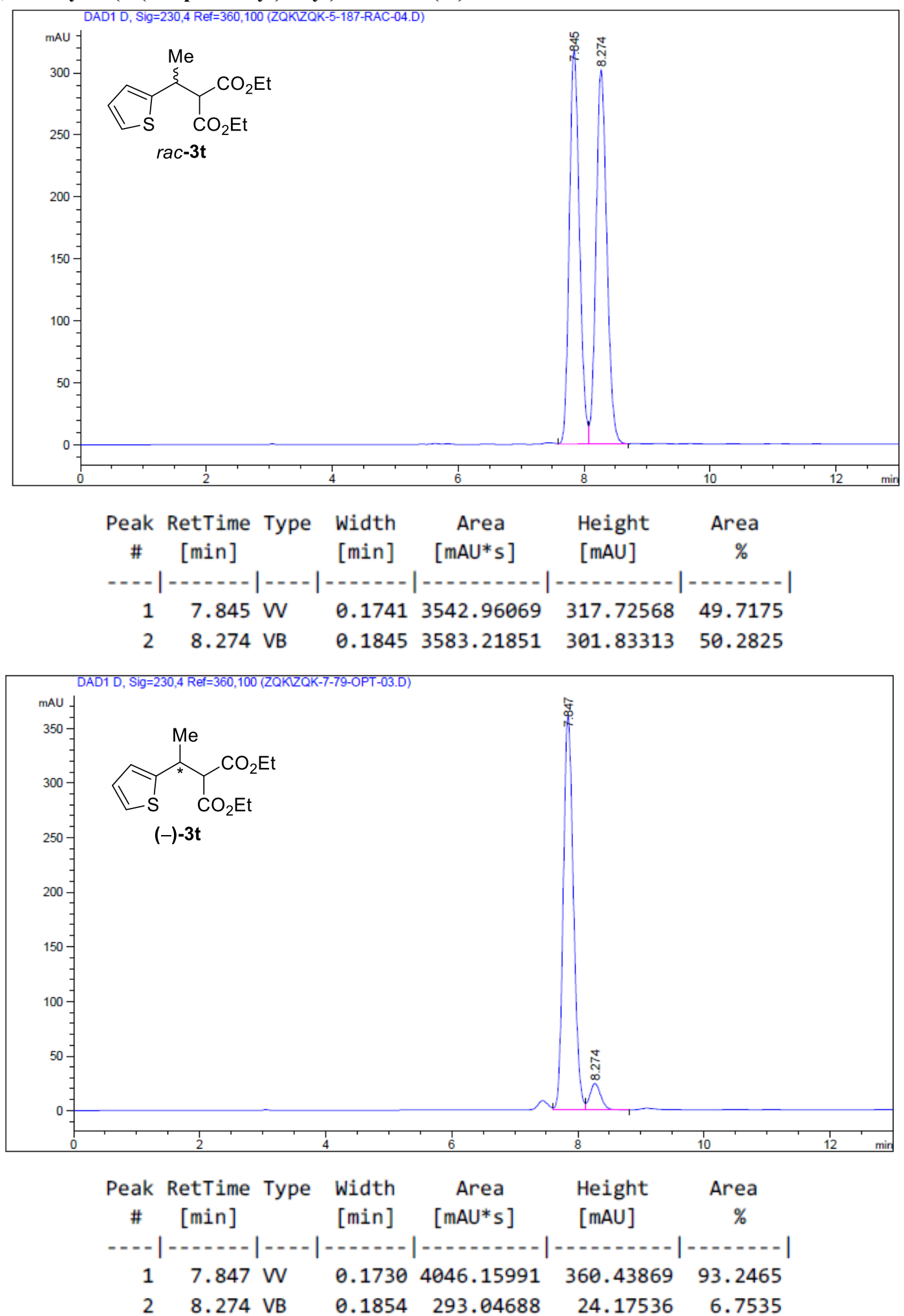
(-)-Diethyl-2-(1-(furan-2-yl)ethyl)malonate (3u)
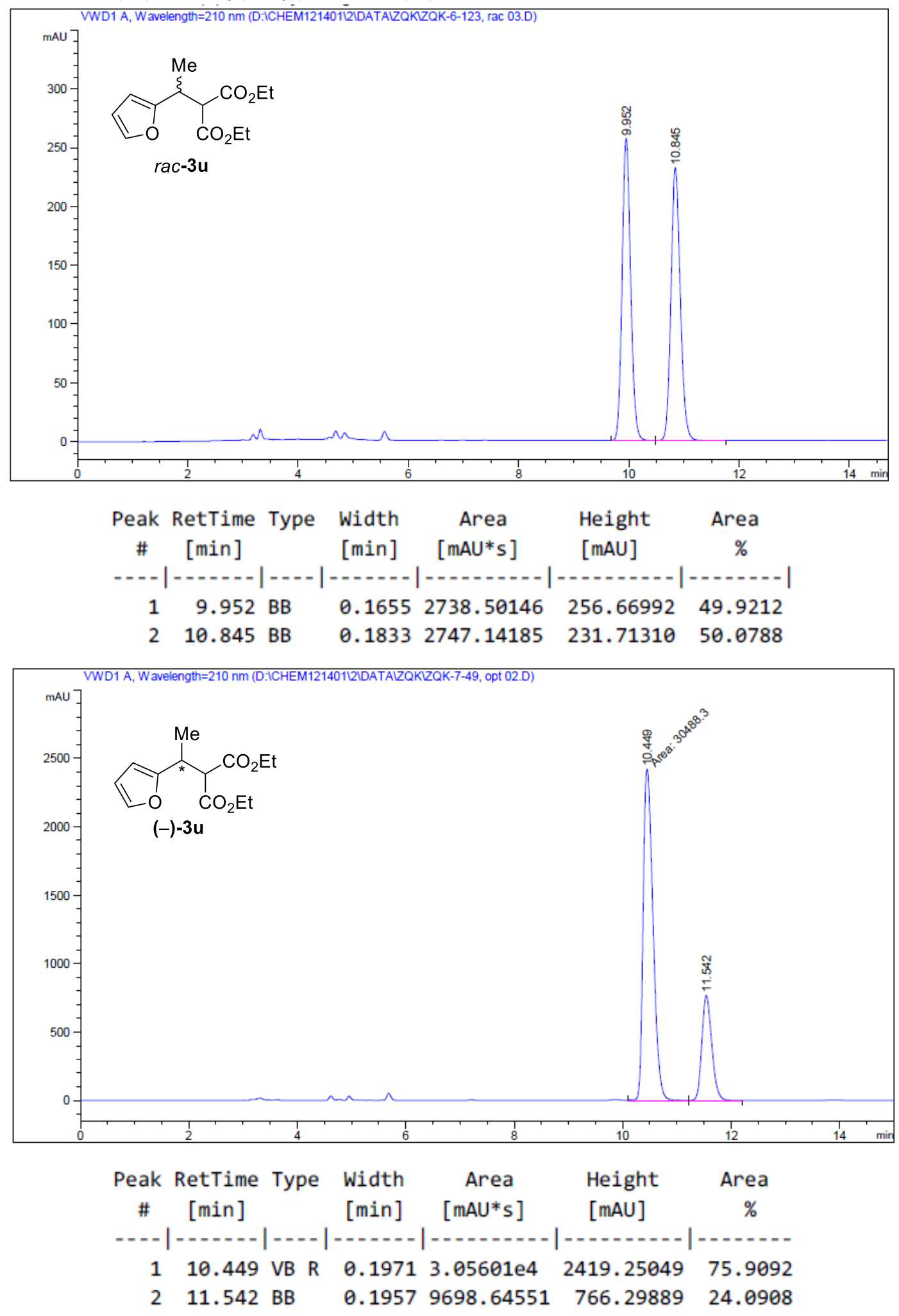


\section{(-)-Diethyl-2-(1-cyclohexylethyl)malonate (3v)}

(Note: The ee value of $\mathbf{3 v}$ was determined by transformation of it into 3-cyclohexyl- $N$ phenylbutanamide).
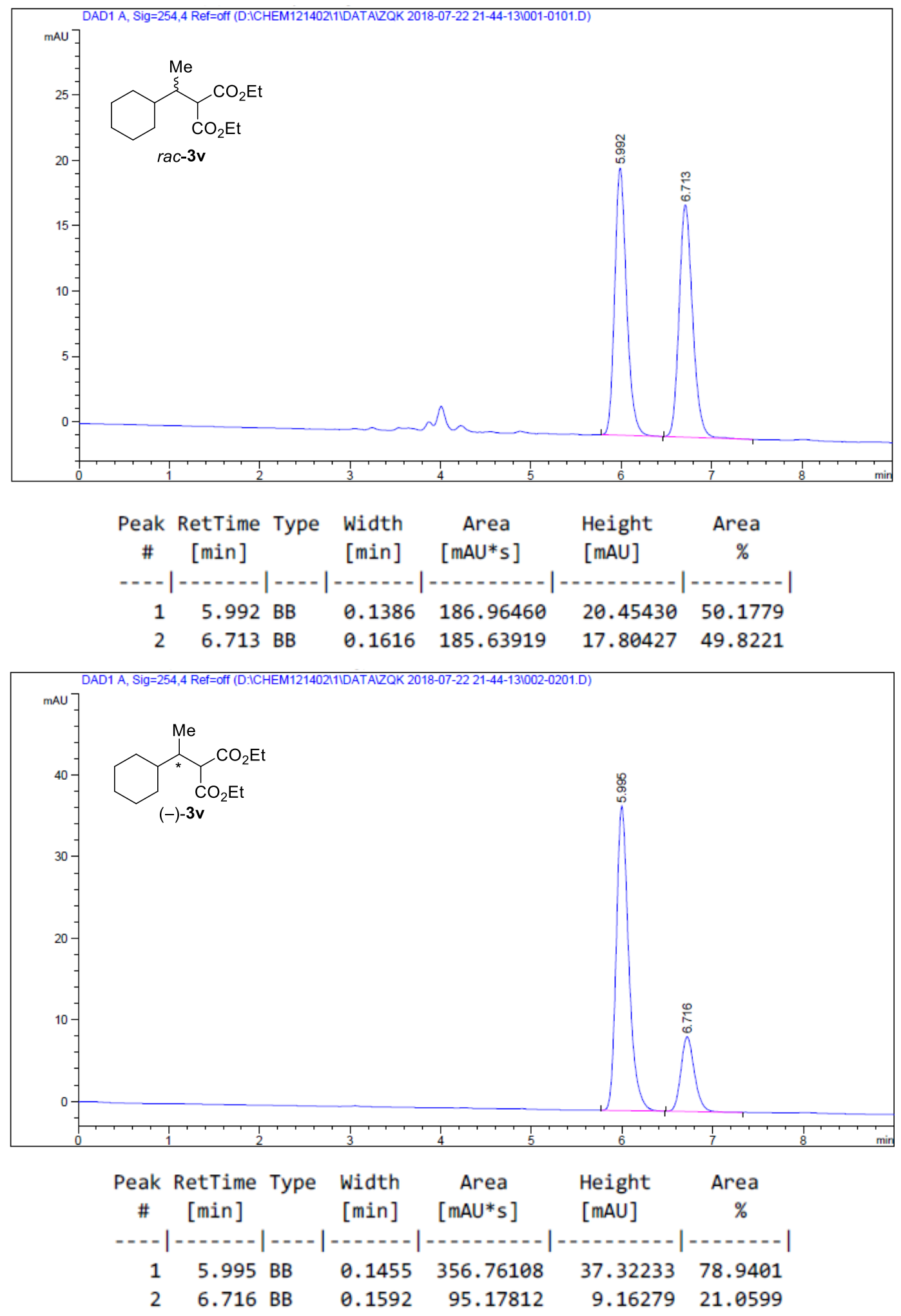
(H). Cartesian Coordinates and Energies of the Optimized Structures.

\section{TS-R (entry 1):}

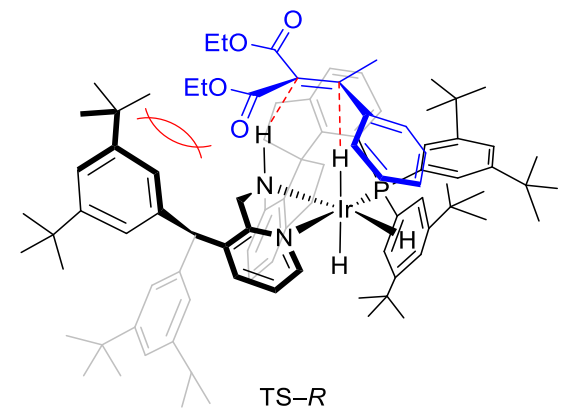

SCF energy [B3LYP-GD3BJ/6-311+G**/CPCM (MeOH)]: -4549.86586614 a.u. Thermal correction to Gibbs free energy at $298 \mathrm{~K}: 1.834940$ a.u.

Gibbs free energy at $298 \mathrm{~K}$ [B3LYP-GD3BJ/6-311+G**/CPCM (MeOH)]:

-4548.03092614 a.u.

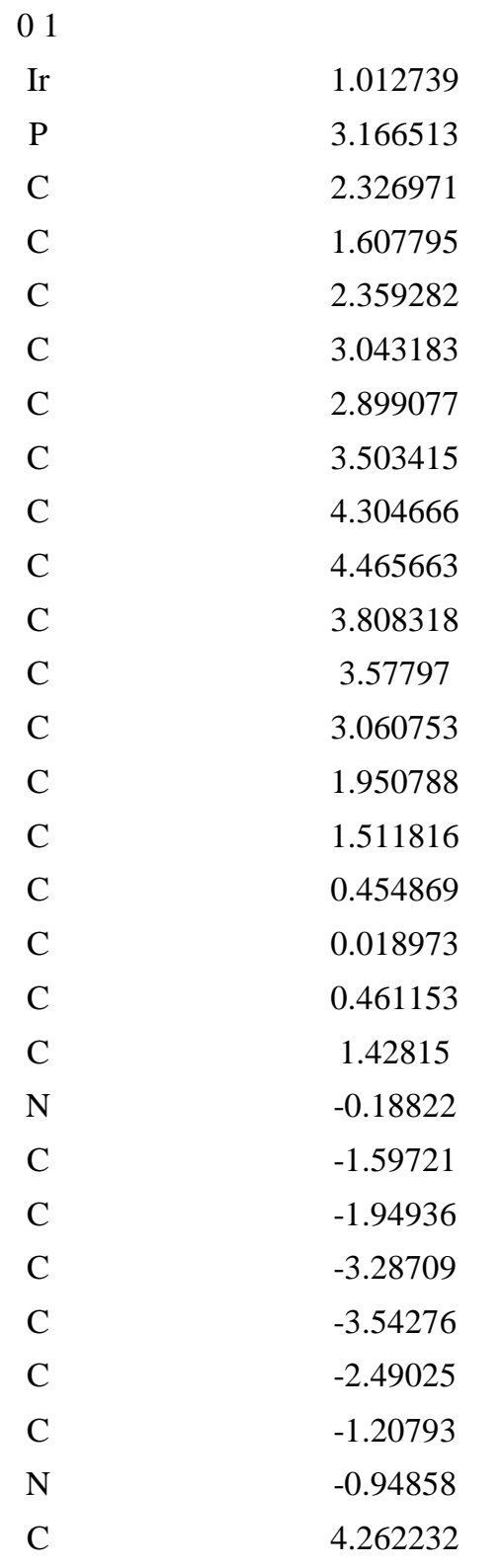

$\begin{array}{ll}-0.55236 & -0.82819 \\ -0.31618 & -0.11129 \\ 1.436091 & 2.870007 \\ 1.440775 & 4.249755\end{array}$

$0.399904 \quad 5.097206$

$-0.47586 \quad 4.076125$

$0.020583 \quad 2.763963$

$-0.67044 \quad 1.685028$

$-1.78807 \quad 1.991263$

$\begin{array}{ll}-2.24866 & 3.299223\end{array}$

$-1.6091 \quad 4.348862$

$2.366924 \quad 3.004882$

$3.767101 \quad 2.709828$

$3.496492 \quad 1.729327$

$2.144479 \quad 1.777227$

$1.752002 \quad 0.920276$

$2.722493 \quad-0.02477$

$4.034519 \quad-0.04174$

$4.44939 \quad 0.870411$

$0.451262 \quad 0.791887$

$\begin{array}{ll}0.561447 & 0.367269\end{array}$

$-0.16024-0.9001$

$-0.22924 \quad-1.33756$

$-0.8376-2.5644$

$-1.38778 \quad-3.29975$

$-1.3247 \quad-2.77964$

$-0.71568-1.60615$

$-1.54314 \quad-0.96458$ 
C

C

C

C

C

C

C

C

C

C

C

C

C

C

C

C

C

C

C

C

C

C

C

C

C

C

C

$\mathrm{H}$

$\mathrm{H}$

H

$\mathrm{H}$

H

H

H

$\mathrm{H}$

$\mathrm{H}$

H

H

$\mathrm{H}$

H

$\mathrm{H}$

H

$\mathrm{H}$

$\mathrm{H}$
5.539282

6.31858

5.78446

4.504662

3.756789

7.730172

8.64602

7.695358

8.323465

3.878468

3.278633

4.903217

2.755469

4.027389

3.503676

4.193824

5.413896

5.963556

5.250132

3.678508

2.333626

3.494184

4.710291

7.302244

7.07114

7.951241

8.275494

1.2089

1.648424

1.629529

0.558646

3.112164

1.677462

4.794317

5.091287

3.9051

4.054701

4.312158

2.665639

3.830953

$-0.6698$

0.05558

1.79645

$-0.25352$
$-1.23859$

$-2.20594$

$-3.49197$

$-3.8313$

$-2.83771$

$-1.83704$

$-1.69476$

$-0.50011$

$-2.90801$

$-5.21767$

$-5.74353$

$-6.24338$

$-5.07635$

1.279334

2.18609

3.357179

3.607981

2.726003

1.550632

4.345666

3.898243

5.738447

4.434876

2.993246

3.076266

4.306182

1.836281

0.650027

$-1.38007$

2.44731

1.160494

0.862953

$-0.18414$

$-2.33266$

$-3.12441$

$-1.97237$

2.254999

2.090338

4.260362

4.431771

2.430521

4.721761

5.476927

$-0.30493$
$-1.44012$

$-2.09582$

$-2.25157$

$-1.78228$

$-1.14493$

$-2.58084$

$-1.34654$

$-3.35005$

$-3.50993$

$-2.00102$

$-0.68038$

$-2.50908$

$-3.05169$

$-0.41986$

$-1.34832$

$-1.67222$

$-1.02045$

$-0.08213$

0.20258

$-2.72972$

$-3.32383$

$-2.09354$

$-3.87426$

0.625906

2.149059

0.162712

0.321839

$-1.88529$

$-2.01606$

4.691885

4.137644

5.759063

5.731857

1.186495

3.487511

5.375412

3.988675

2.244697

3.615564

2.288442

$-0.81163$

$-0.78553$

0.89063

1.468019 


\begin{tabular}{|c|c|c|c|}
\hline $\mathrm{H}$ & -1.88025 & 1.607492 & 0.259365 \\
\hline $\mathrm{H}$ & -2.22895 & 0.221176 & 1.193189 \\
\hline $\mathrm{H}$ & -4.56741 & -0.90328 & -2.93203 \\
\hline $\mathrm{H}$ & 5.931572 & -0.23283 & -1.30906 \\
\hline $\mathrm{H}$ & 6.375386 & -4.24934 & -2.75893 \\
\hline $\mathrm{H}$ & 2.749823 & -3.05497 & -0.79502 \\
\hline $\mathrm{H}$ & 9.668201 & -1.41267 & -1.64868 \\
\hline $\mathrm{H}$ & 8.700809 & -2.64329 & -0.78982 \\
\hline $\mathrm{H}$ & 8.269876 & -0.92333 & -0.6603 \\
\hline $\mathrm{H}$ & 7.023603 & -0.56439 & -4.22024 \\
\hline $\mathrm{H}$ & 8.703851 & -0.24379 & -3.71237 \\
\hline $\mathrm{H}$ & 7.349126 & 0.332597 & -2.72099 \\
\hline $\mathrm{H}$ & 7.686084 & -3.07739 & -4.39171 \\
\hline $\mathrm{H}$ & 8.460063 & -3.87118 & -2.99545 \\
\hline $\mathrm{H}$ & 9.313783 & -2.58454 & -3.86616 \\
\hline $\mathrm{H}$ & 4.049145 & -5.80592 & 0.104388 \\
\hline $\mathrm{H}$ & 2.855939 & -6.74989 & -0.82845 \\
\hline $\mathrm{H}$ & 2.463828 & -5.10526 & -0.31222 \\
\hline $\mathrm{H}$ & 5.744344 & -6.35766 & -1.80732 \\
\hline $\mathrm{H}$ & 5.312505 & -5.96587 & -3.49238 \\
\hline $\mathrm{H}$ & 4.421577 & -7.22713 & -2.62017 \\
\hline $\mathrm{H}$ & 2.253782 & -6.04397 & -3.21664 \\
\hline $\mathrm{H}$ & 3.163037 & -4.72789 & -4.01354 \\
\hline $\mathrm{H}$ & 1.997438 & -4.35063 & -2.72495 \\
\hline $\mathrm{H}$ & 2.546927 & 1.947963 & -1.80664 \\
\hline $\mathrm{H}$ & 5.951562 & 4.522531 & -1.26419 \\
\hline $\mathrm{H}$ & 5.648901 & 0.832344 & 0.920995 \\
\hline $\mathrm{H}$ & 1.991673 & 4.635745 & -4.06663 \\
\hline $\mathrm{H}$ & 1.558714 & 3.810674 & -2.54977 \\
\hline $\mathrm{H}$ & 2.416307 & 2.925114 & -3.83074 \\
\hline $\mathrm{H}$ & 3.129989 & 6.458466 & -2.84428 \\
\hline $\mathrm{H}$ & 4.438383 & 6.130397 & -1.68584 \\
\hline $\mathrm{H}$ & 4.864509 & 3.448201 & -4.33836 \\
\hline $\mathrm{H}$ & 5.68695 & 4.795339 & -3.51715 \\
\hline $\mathrm{H}$ & 4.360144 & 5.13067 & -4.65413 \\
\hline $\mathrm{H}$ & 6.366769 & 3.885105 & 2.396706 \\
\hline $\mathrm{H}$ & 8.020997 & 3.276171 & 2.670771 \\
\hline $\mathrm{H}$ & 6.659666 & 2.139327 & 2.551438 \\
\hline $\mathrm{H}$ & 8.162699 & 4.298387 & -0.91758 \\
\hline $\mathrm{H}$ & 8.907168 & 4.45442 & 0.688251 \\
\hline $\mathrm{H}$ & 7.313578 & 5.17643 & 0.381428 \\
\hline $\mathrm{H}$ & 7.887481 & 0.87473 & 0.688254 \\
\hline $\mathrm{H}$ & 9.248147 & 2.010145 & 0.809783 \\
\hline $\mathrm{H}$ & 8.447615 & 1.739346 & -0.76128 \\
\hline
\end{tabular}




\begin{tabular}{|c|c|c|c|}
\hline $\mathrm{H}$ & 2.764223 & 5.694322 & -1.273 \\
\hline $\mathrm{H}$ & 0.818772 & -1.91671 & 0.185803 \\
\hline $\mathrm{H}$ & -2.66416 & -1.87553 & -4.2599 \\
\hline $\mathrm{H}$ & -0.34819 & -1.75148 & -3.29388 \\
\hline $\mathrm{C}$ & -4.38643 & 0.309885 & -0.42947 \\
\hline $\mathrm{H}$ & -4.14946 & -0.10039 & 0.561949 \\
\hline $\mathrm{C}$ & -5.75538 & -0.25286 & -0.76903 \\
\hline $\mathrm{C}$ & -5.91805 & -1.64192 & -0.67619 \\
\hline $\mathrm{C}$ & -6.8379 & 0.53863 & -1.14508 \\
\hline $\mathrm{C}$ & -7.14292 & -2.25128 & -0.95729 \\
\hline $\mathrm{H}$ & -5.05228 & -2.24346 & -0.3918 \\
\hline $\mathrm{C}$ & -8.08704 & -0.03528 & -1.43752 \\
\hline $\mathrm{H}$ & -6.70314 & 1.618794 & -1.20401 \\
\hline $\mathrm{C}$ & -8.21704 & -1.42709 & -1.33678 \\
\hline $\mathrm{H}$ & -9.17715 & -1.88401 & -1.55908 \\
\hline $\mathrm{C}$ & -4.28163 & 1.817747 & -0.27774 \\
\hline $\mathrm{C}$ & -4.14445 & 2.354266 & 1.005316 \\
\hline $\mathrm{C}$ & -4.15558 & 2.653583 & -1.39058 \\
\hline $\mathrm{C}$ & -3.80348 & 3.69577 & 1.203231 \\
\hline $\mathrm{H}$ & -4.21968 & 1.677536 & 1.85516 \\
\hline $\mathrm{C}$ & -3.869 & 4.017481 & -1.23736 \\
\hline $\mathrm{H}$ & -4.24423 & 2.213483 & -2.38283 \\
\hline $\mathrm{C}$ & -3.68098 & 4.506545 & 0.064712 \\
\hline $\mathrm{H}$ & -3.40233 & 5.553085 & 0.196482 \\
\hline $\mathrm{C}$ & -7.25077 & -3.78104 & -0.86295 \\
\hline $\mathrm{C}$ & -6.33607 & -4.40812 & -1.9374 \\
\hline $\mathrm{C}$ & -6.783 & -4.24641 & 0.532731 \\
\hline $\mathrm{C}$ & -8.68647 & -4.27805 & -1.08725 \\
\hline $\mathrm{H}$ & -6.6427 & -4.08897 & -2.94617 \\
\hline $\mathrm{H}$ & -5.28558 & -4.11453 & -1.79267 \\
\hline $\mathrm{H}$ & -6.38797 & -5.50848 & -1.89514 \\
\hline $\mathrm{H}$ & -7.39389 & -3.78561 & 1.325318 \\
\hline $\mathrm{H}$ & -6.8756 & -5.34098 & 0.621286 \\
\hline $\mathrm{H}$ & -5.7307 & -3.98612 & 0.717619 \\
\hline $\mathrm{H}$ & -8.72128 & -5.37451 & -0.99351 \\
\hline $\mathrm{H}$ & -9.38338 & -3.856 & -0.34647 \\
\hline $\mathrm{H}$ & -9.05726 & -4.0205 & -2.09124 \\
\hline $\mathrm{C}$ & -9.25131 & 0.880246 & -1.8477 \\
\hline $\mathrm{C}$ & -9.55127 & 1.863146 & -0.69602 \\
\hline $\mathrm{C}$ & -8.85301 & 1.675064 & -3.10961 \\
\hline $\mathrm{C}$ & -10.5328 & 0.092392 & -2.15844 \\
\hline $\mathrm{H}$ & -9.83638 & 1.318509 & 0.217682 \\
\hline $\mathrm{H}$ & -8.67748 & 2.486737 & -0.45547 \\
\hline $\mathrm{H}$ & -10.3812 & 2.535518 & -0.96849 \\
\hline
\end{tabular}




\begin{tabular}{|c|c|c|c|}
\hline $\mathrm{H}$ & -8.62848 & 0.994408 & -3.94588 \\
\hline $\mathrm{H}$ & -9.67375 & 2.341799 & -3.42056 \\
\hline $\mathrm{H}$ & -7.96312 & 2.298036 & -2.9349 \\
\hline $\mathrm{H}$ & -11.3352 & 0.786703 & -2.45222 \\
\hline $\mathrm{H}$ & -10.3852 & -0.61644 & -2.98772 \\
\hline $\mathrm{H}$ & -10.8871 & -0.47328 & -1.2831 \\
\hline $\mathrm{C}$ & -3.47579 & 4.254767 & 2.596139 \\
\hline $\mathrm{C}$ & -3.68771 & 3.208095 & 3.703047 \\
\hline $\mathrm{C}$ & -1.99052 & 4.677829 & 2.619076 \\
\hline $\mathrm{C}$ & -4.37263 & 5.473717 & 2.889981 \\
\hline $\mathrm{H}$ & -4.72947 & 2.851325 & 3.733512 \\
\hline $\mathrm{H}$ & -3.02373 & 2.338206 & 3.582731 \\
\hline $\mathrm{H}$ & -3.46285 & 3.657971 & 4.682338 \\
\hline $\mathrm{H}$ & -1.77232 & 5.44956 & 1.866302 \\
\hline $\mathrm{H}$ & -1.72527 & 5.084815 & 3.608371 \\
\hline $\mathrm{H}$ & -1.33218 & 3.821138 & 2.412149 \\
\hline $\mathrm{H}$ & -4.1402 & 5.890779 & 3.883099 \\
\hline $\mathrm{H}$ & -4.22917 & 6.275598 & 2.149998 \\
\hline $\mathrm{H}$ & -5.43712 & 5.190648 & 2.877507 \\
\hline $\mathrm{C}$ & -3.70392 & 4.961467 & -2.43896 \\
\hline $\mathrm{C}$ & -2.24874 & 5.473868 & -2.48427 \\
\hline $\mathrm{C}$ & -4.00952 & 4.259808 & -3.77146 \\
\hline $\mathrm{C}$ & -4.66851 & 6.155995 & -2.28938 \\
\hline $\mathrm{H}$ & -1.97928 & 6.01294 & -1.56394 \\
\hline $\mathrm{H}$ & -1.54393 & 4.63619 & -2.60382 \\
\hline $\mathrm{H}$ & -2.1073 & 6.162609 & -3.33296 \\
\hline $\mathrm{H}$ & -5.03757 & 3.866501 & -3.7977 \\
\hline $\mathrm{H}$ & -3.90309 & 4.974583 & -4.60202 \\
\hline $\mathrm{H}$ & -3.31834 & 3.424992 & -3.96289 \\
\hline $\mathrm{H}$ & -4.56613 & 6.842962 & -3.14501 \\
\hline $\mathrm{H}$ & -5.71375 & 5.811503 & -2.249 \\
\hline $\mathrm{H}$ & -4.46951 & 6.730792 & -1.37289 \\
\hline $\mathrm{C}$ & -1.67827 & -5.92656 & 0.694464 \\
\hline $\mathrm{C}$ & -1.41734 & -6.12772 & -0.6646 \\
\hline $\mathrm{C}$ & -0.68258 & -5.16972 & -1.3638 \\
\hline $\mathrm{C}$ & -0.21885 & -4.02158 & -0.71633 \\
\hline $\mathrm{C}$ & -0.48301 & -3.80088 & 0.638733 \\
\hline $\mathrm{C}$ & -1.2139 & -4.77939 & 1.335293 \\
\hline $\mathrm{C}$ & 0.08035 & -2.63028 & 1.42601 \\
\hline $\mathrm{C}$ & -0.81721 & -1.6851 & 2.071266 \\
\hline $\mathrm{C}$ & -0.62116 & -1.02233 & 3.38702 \\
\hline $\mathrm{C}$ & -2.25113 & -1.88968 & 1.7777 \\
\hline $\mathrm{O}$ & -2.73706 & -2.32523 & 0.747316 \\
\hline $\mathrm{O}$ & -1.03613 & 0.100281 & 3.591382 \\
\hline
\end{tabular}




$\begin{array}{lccc}\mathrm{C} & 1.346238 & -3.0637 & 2.138953 \\ \mathrm{O} & -3.0458 & -1.59899 & 2.850825 \\ \mathrm{C} & -5.14134 & -1.01371 & 3.816426 \\ \mathrm{C} & -4.45011 & -1.83605 & 2.748799 \\ \mathrm{O} & 0.069208 & -1.64349 & 4.388051 \\ \mathrm{C} & -1.47425 & -2.81882 & 5.843051 \\ \mathrm{C} & -0.35139 & -2.93598 & 4.826563 \\ \mathrm{H} & -2.25136 & -6.66619 & 1.259344 \\ \mathrm{H} & -1.78395 & -7.02393 & -1.17089 \\ \mathrm{H} & -0.4653 & -5.31102 & -2.42523 \\ \mathrm{H} & 0.345347 & -3.26621 & -1.26258 \\ \mathrm{H} & -1.44179 & -4.62977 & 2.393017 \\ \mathrm{H} & 2.090278 & -3.42213 & 1.420242 \\ \mathrm{H} & 1.776805 & -2.26909 & 2.744796 \\ \mathrm{H} & 1.108922 & -3.91137 & 2.800068 \\ \mathrm{H} & -6.2296 & -1.17418 & 3.771179 \\ \mathrm{H} & -4.78942 & -1.28862 & 4.822531 \\ \mathrm{H} & -4.9405 & 0.058757 & 3.670168 \\ \mathrm{H} & -4.8068 & -1.5711 & 1.74546 \\ \mathrm{H} & -4.64106 & -2.91549 & 2.877738 \\ \mathrm{H} & -1.75719 & -3.81689 & 6.215278 \\ \mathrm{H} & -1.16499 & -2.19929 & 6.698284 \\ \mathrm{H} & -2.357 & -2.35482 & 5.378492 \\ \mathrm{H} & -0.67546 & -3.53881 & 3.966571 \\ \mathrm{H} & 0.542001 & -3.41184 & 5.257378\end{array}$

\section{TS-S (entry 2):}

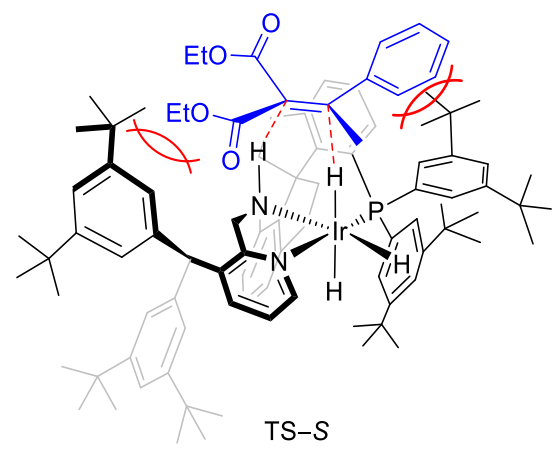

SCF energy [B3LYP-GD3BJ/6-311+G**/CPCM (MeOH)]: -4549.86946410 a.u.

Thermal correction to Gibbs free energy at $298 \mathrm{~K}: 1.84318300$ a.u.

Gibbs free energy at $298 \mathrm{~K}$ [B3LYP-GD3BJ/6-311+G**/CPCM $(\mathrm{MeOH})]$ :

-4548.02628110 a.u.

$\begin{array}{cccc}01 & & & \\ \mathrm{Ir} & 0.987685 & -0.32061 & -0.95743 \\ \mathrm{P} & 3.144828 & -0.11373 & -0.21618\end{array}$




\begin{tabular}{|c|c|c|c|}
\hline C & 2.254822 & 0.384688 & 3.17759 \\
\hline $\mathrm{C}$ & 1.554152 & -0.28861 & 4.388531 \\
\hline $\mathrm{C}$ & 2.582305 & -1.3117 & 4.892854 \\
\hline $\mathrm{C}$ & 3.344699 & -1.66978 & 3.639961 \\
\hline $\mathrm{C}$ & 3.038405 & -0.79786 & 2.575229 \\
\hline $\mathrm{C}$ & 3.613609 & -1.03489 & 1.31015 \\
\hline $\mathrm{C}$ & 4.504296 & -2.11851 & 1.174154 \\
\hline $\mathrm{C}$ & 4.819175 & -2.95002 & 2.247169 \\
\hline $\mathrm{C}$ & 4.22983 & -2.73096 & 3.490972 \\
\hline $\mathrm{C}$ & 3.361174 & 1.340468 & 3.742058 \\
\hline $\mathrm{C}$ & 2.678617 & 2.67561 & 3.97444 \\
\hline $\mathrm{C}$ & 1.63527 & 2.685244 & 2.892901 \\
\hline $\mathrm{C}$ & 1.341477 & 1.375773 & 2.420835 \\
\hline $\mathrm{C}$ & 0.30283 & 1.246052 & 1.463625 \\
\hline $\mathrm{C}$ & -0.22183 & 2.449348 & 0.918192 \\
\hline $\mathrm{C}$ & 0.116361 & 3.711244 & 1.377104 \\
\hline $\mathrm{C}$ & 1.035799 & 3.839803 & 2.414441 \\
\hline $\mathrm{N}$ & -0.25697 & 0.030822 & 0.914557 \\
\hline $\mathrm{C}$ & -1.6627 & 0.184235 & 0.483515 \\
\hline $\mathrm{C}$ & -1.95779 & 0.031989 & -0.98047 \\
\hline $\mathrm{C}$ & -3.28691 & 0.069199 & -1.43382 \\
\hline $\mathrm{C}$ & -3.5183 & -0.14108 & -2.79441 \\
\hline $\mathrm{C}$ & -2.44298 & -0.38189 & -3.65067 \\
\hline $\mathrm{C}$ & -1.16083 & -0.43101 & -3.12113 \\
\hline $\mathrm{N}$ & -0.93201 & -0.23591 & -1.80918 \\
\hline $\mathrm{C}$ & 4.357918 & -0.70729 & -1.49618 \\
\hline $\mathrm{C}$ & 5.598277 & -0.0924 & -1.69691 \\
\hline $\mathrm{C}$ & 6.465321 & -0.51158 & -2.71679 \\
\hline $\mathrm{C}$ & 6.059761 & -1.5734 & -3.53613 \\
\hline $\mathrm{C}$ & 4.82812 & -2.22064 & -3.35855 \\
\hline $\mathrm{C}$ & 3.992913 & -1.77447 & -2.32761 \\
\hline $\mathrm{C}$ & 7.820353 & 0.193578 & -2.88963 \\
\hline $\mathrm{C}$ & 8.711346 & -0.1576 & -1.6794 \\
\hline $\mathrm{C}$ & 7.611611 & 1.721304 & -2.95444 \\
\hline $\mathrm{C}$ & 8.545774 & -0.24434 & -4.17143 \\
\hline $\mathrm{C}$ & 4.347023 & -3.3588 & -4.2727 \\
\hline $\mathrm{C}$ & 4.091204 & -4.62198 & -3.4269 \\
\hline $\mathrm{C}$ & 5.372425 & -3.70072 & -5.36397 \\
\hline $\mathrm{C}$ & 3.030933 & -2.928 & -4.95597 \\
\hline $\mathrm{C}$ & 3.767701 & 1.593933 & 0.108663 \\
\hline $\mathrm{C}$ & 3.149518 & 2.698582 & -0.48856 \\
\hline $\mathrm{C}$ & 3.692137 & 3.979891 & -0.36627 \\
\hline $\mathrm{C}$ & 4.867165 & 4.131706 & 0.389945 \\
\hline $\mathrm{C}$ & 5.513822 & 3.052111 & 1.002934 \\
\hline
\end{tabular}




\begin{tabular}{|c|c|c|c|}
\hline C & 4.943009 & 1.777665 & 0.846529 \\
\hline $\mathrm{C}$ & 3.073127 & 5.201792 & -1.06294 \\
\hline $\mathrm{C}$ & 1.791213 & 4.837042 & -1.82758 \\
\hline $\mathrm{C}$ & 2.728488 & 6.281557 & -0.01704 \\
\hline $\mathrm{C}$ & 4.095036 & 5.770071 & -2.07078 \\
\hline $\mathrm{C}$ & 6.820771 & 3.214743 & 1.799828 \\
\hline $\mathrm{C}$ & 6.618324 & 2.730733 & 3.250035 \\
\hline $\mathrm{C}$ & 7.293838 & 4.675789 & 1.842023 \\
\hline $\mathrm{C}$ & 7.924667 & 2.366646 & 1.136652 \\
\hline $\mathrm{H}$ & 1.182842 & 1.142674 & -1.58685 \\
\hline $\mathrm{H}$ & 1.626689 & -0.71575 & -2.33824 \\
\hline $\mathrm{H}$ & 1.249959 & 0.449398 & 5.145129 \\
\hline $\mathrm{H}$ & 0.650644 & -0.80925 & 4.05373 \\
\hline $\mathrm{H}$ & 3.251359 & -0.87212 & 5.652433 \\
\hline $\mathrm{H}$ & 2.118616 & -2.19208 & 5.357416 \\
\hline $\mathrm{H}$ & 4.95256 & -2.32134 & 0.202172 \\
\hline $\mathrm{H}$ & 5.508823 & -3.78414 & 2.099275 \\
\hline $\mathrm{H}$ & 4.436879 & -3.39718 & 4.332286 \\
\hline $\mathrm{H}$ & 3.852055 & 0.922335 & 4.629539 \\
\hline $\mathrm{H}$ & 4.124741 & 1.465855 & 2.972368 \\
\hline $\mathrm{H}$ & 2.200099 & 2.727694 & 4.969233 \\
\hline $\mathrm{H}$ & 3.365037 & 3.533226 & 3.900633 \\
\hline $\mathrm{H}$ & -0.89431 & 2.39418 & 0.067057 \\
\hline $\mathrm{H}$ & -0.33152 & 4.58936 & 0.908674 \\
\hline $\mathrm{H}$ & 1.311781 & 4.815081 & 2.819975 \\
\hline $\mathrm{H}$ & -0.25235 & -0.88642 & 1.352632 \\
\hline $\mathrm{H}$ & -2.08479 & 1.126169 & 0.849057 \\
\hline $\mathrm{H}$ & -2.25449 & -0.58407 & 0.977514 \\
\hline $\mathrm{H}$ & -4.54179 & -0.1322 & -3.17278 \\
\hline $\mathrm{H}$ & 5.888943 & 0.742586 & -1.0651 \\
\hline $\mathrm{H}$ & 6.71407 & -1.8982 & -4.33994 \\
\hline $\mathrm{H}$ & 3.018447 & -2.23624 & -2.18892 \\
\hline $\mathrm{H}$ & 9.687026 & 0.350608 & -1.7522 \\
\hline $\mathrm{H}$ & 8.891183 & -1.24289 & -1.63043 \\
\hline $\mathrm{H}$ & 8.240099 & 0.146899 & -0.73498 \\
\hline $\mathrm{H}$ & 6.973062 & 1.992903 & -3.80955 \\
\hline $\mathrm{H}$ & 8.579841 & 2.234214 & -3.07212 \\
\hline $\mathrm{H}$ & 7.134785 & 2.114963 & -2.04558 \\
\hline $\mathrm{H}$ & 7.942823 & -0.03976 & -5.06973 \\
\hline $\mathrm{H}$ & 8.791004 & -1.31718 & -4.1598 \\
\hline $\mathrm{H}$ & 9.493325 & 0.307758 & -4.26949 \\
\hline $\mathrm{H}$ & 5.009454 & -4.93959 & -2.90773 \\
\hline $\mathrm{H}$ & 3.756036 & -5.45367 & -4.06767 \\
\hline $\mathrm{H}$ & 3.315754 & -4.45557 & -2.66733 \\
\hline
\end{tabular}




\begin{tabular}{|c|c|c|c|}
\hline $\mathrm{H}$ & 6.328578 & -4.03795 & -4.93455 \\
\hline $\mathrm{H}$ & 5.575409 & -2.84053 & -6.02049 \\
\hline $\mathrm{H}$ & 4.985913 & -4.51628 & -5.99444 \\
\hline $\mathrm{H}$ & 2.660299 & -3.72961 & -5.6154 \\
\hline $\mathrm{H}$ & 3.184042 & -2.02313 & -5.56495 \\
\hline $\mathrm{H}$ & 2.24524 & -2.70729 & -4.21808 \\
\hline $\mathrm{H}$ & 2.237806 & 2.52858 & -1.05468 \\
\hline $\mathrm{H}$ & 5.292221 & 5.128709 & 0.489038 \\
\hline $\mathrm{H}$ & 5.425347 & 0.90794 & 1.295354 \\
\hline $\mathrm{H}$ & 1.36669 & 5.738796 & -2.29557 \\
\hline $\mathrm{H}$ & 1.02906 & 4.411943 & -1.16076 \\
\hline $\mathrm{H}$ & 1.986753 & 4.106225 & -2.62658 \\
\hline $\mathrm{H}$ & 2.290882 & 7.165808 & -0.50815 \\
\hline $\mathrm{H}$ & 3.61944 & 6.614577 & 0.536445 \\
\hline $\mathrm{H}$ & 4.363281 & 5.012326 & -2.82342 \\
\hline $\mathrm{H}$ & 5.022128 & 6.09337 & -1.57363 \\
\hline $\mathrm{H}$ & 3.673244 & 6.642562 & -2.59598 \\
\hline $\mathrm{H}$ & 5.822618 & 3.303029 & 3.750569 \\
\hline $\mathrm{H}$ & 7.547748 & 2.859614 & 3.827591 \\
\hline $\mathrm{H}$ & 6.346202 & 1.666496 & 3.292574 \\
\hline $\mathrm{H}$ & 7.490974 & 5.071299 & 0.833908 \\
\hline $\mathrm{H}$ & 8.230629 & 4.746695 & 2.415792 \\
\hline $\mathrm{H}$ & 6.554994 & 5.330376 & 2.329435 \\
\hline $\mathrm{H}$ & 7.661958 & 1.298769 & 1.127004 \\
\hline $\mathrm{H}$ & 8.874392 & 2.470522 & 1.685783 \\
\hline $\mathrm{H}$ & 8.094527 & 2.681907 & 0.096123 \\
\hline $\mathrm{H}$ & 2.002811 & 5.896264 & 0.712991 \\
\hline $\mathrm{H}$ & 0.864642 & -1.93788 & -0.41284 \\
\hline $\mathrm{H}$ & -2.59525 & -0.55085 & -4.71762 \\
\hline $\mathrm{H}$ & -0.28343 & -0.64025 & -3.73145 \\
\hline $\mathrm{C}$ & -4.42269 & 0.324162 & -0.45502 \\
\hline $\mathrm{H}$ & -4.08882 & -0.03109 & 0.530608 \\
\hline $\mathrm{C}$ & -5.62651 & -0.5476 & -0.78557 \\
\hline $\mathrm{C}$ & -5.59137 & -1.88377 & -0.37642 \\
\hline $\mathrm{C}$ & -6.72423 & -0.07529 & -1.50659 \\
\hline $\mathrm{C}$ & -6.64088 & -2.76052 & -0.6759 \\
\hline $\mathrm{H}$ & -4.72815 & -2.24388 & 0.188255 \\
\hline $\mathrm{C}$ & -7.80413 & -0.91715 & -1.81074 \\
\hline $\mathrm{H}$ & -6.74429 & 0.97204 & -1.81081 \\
\hline $\mathrm{C}$ & -7.73911 & -2.25414 & -1.39028 \\
\hline $\mathrm{H}$ & -8.56813 & -2.91803 & -1.61829 \\
\hline $\mathrm{C}$ & -4.7312 & 1.810123 & -0.28239 \\
\hline $\mathrm{C}$ & -5.81777 & 2.183578 & 0.520154 \\
\hline $\mathrm{C}$ & -3.94105 & 2.804917 & -0.86395 \\
\hline
\end{tabular}




\begin{tabular}{|c|c|c|c|}
\hline C & -6.14396 & 3.527677 & 0.721901 \\
\hline $\mathrm{H}$ & -6.4219 & 1.396023 & 0.966653 \\
\hline $\mathrm{C}$ & -4.214 & 4.166139 & -0.65955 \\
\hline $\mathrm{H}$ & -3.10177 & 2.511767 & -1.48945 \\
\hline $\mathrm{C}$ & -5.32372 & 4.499599 & 0.125068 \\
\hline $\mathrm{H}$ & -5.56242 & 5.553153 & 0.277832 \\
\hline $\mathrm{C}$ & -6.54417 & -4.22041 & -0.20886 \\
\hline $\mathrm{C}$ & -5.30233 & -4.87177 & -0.8569 \\
\hline $\mathrm{C}$ & -6.38296 & -4.25286 & 1.326872 \\
\hline $\mathrm{C}$ & -7.78754 & -5.03513 & -0.59366 \\
\hline $\mathrm{H}$ & -5.36466 & -4.82947 & -1.95616 \\
\hline $\mathrm{H}$ & -4.37731 & -4.37211 & -0.53711 \\
\hline $\mathrm{H}$ & -5.23012 & -5.93065 & -0.55872 \\
\hline $\mathrm{H}$ & -7.23543 & -3.76012 & 1.821395 \\
\hline $\mathrm{H}$ & -6.33927 & -5.29627 & 1.68013 \\
\hline $\mathrm{H}$ & -5.45601 & -3.75484 & 1.645253 \\
\hline $\mathrm{H}$ & -7.67673 & -6.07152 & -0.23926 \\
\hline $\mathrm{H}$ & -8.70295 & -4.62455 & -0.13928 \\
\hline $\mathrm{H}$ & -7.93289 & -5.07154 & -1.68479 \\
\hline $\mathrm{C}$ & -9.01139 & -0.33846 & -2.56557 \\
\hline $\mathrm{C}$ & -9.6177 & 0.813676 & -1.73466 \\
\hline C & -8.54779 & 0.204385 & -3.93364 \\
\hline $\mathrm{C}$ & -10.1063 & -1.38803 & -2.80852 \\
\hline $\mathrm{H}$ & -9.95176 & 0.450251 & -0.75009 \\
\hline $\mathrm{H}$ & -8.88886 & 1.620232 & -1.56363 \\
\hline $\mathrm{H}$ & -10.4875 & 1.249286 & -2.2531 \\
\hline $\mathrm{H}$ & -8.10667 & -0.59985 & -4.54338 \\
\hline $\mathrm{H}$ & -9.39904 & 0.629127 & -4.49042 \\
\hline $\mathrm{H}$ & -7.79231 & 0.996541 & -3.82236 \\
\hline $\mathrm{H}$ & -10.9488 & -0.93024 & -3.34986 \\
\hline $\mathrm{H}$ & -9.73746 & -2.22906 & -3.41545 \\
\hline $\mathrm{H}$ & -10.4986 & -1.79434 & -1.86381 \\
\hline $\mathrm{C}$ & -7.36558 & 3.961918 & 1.546665 \\
\hline $\mathrm{C}$ & -8.13007 & 2.760801 & 2.124682 \\
\hline $\mathrm{C}$ & -6.90578 & 4.857085 & 2.715713 \\
\hline $\mathrm{C}$ & -8.32845 & 4.752773 & 0.635579 \\
\hline $\mathrm{H}$ & -8.50774 & 2.097016 & 1.33203 \\
\hline $\mathrm{H}$ & -7.50032 & 2.162367 & 2.800581 \\
\hline $\mathrm{H}$ & -8.99666 & 3.115487 & 2.703723 \\
\hline $\mathrm{H}$ & -6.38916 & 5.761142 & 2.360261 \\
\hline $\mathrm{H}$ & -7.77109 & 5.180103 & 3.316883 \\
\hline $\mathrm{H}$ & -6.21323 & 4.31235 & 3.376249 \\
\hline $\mathrm{H}$ & -9.21638 & 5.076832 & 1.202311 \\
\hline $\mathrm{H}$ & -7.85018 & 5.650954 & 0.217107 \\
\hline
\end{tabular}




\begin{tabular}{|c|c|c|c|}
\hline $\mathrm{H}$ & -8.66835 & 4.129247 & -0.20631 \\
\hline $\mathrm{C}$ & -3.34375 & 5.275953 & -1.27108 \\
\hline $\mathrm{C}$ & -2.82226 & 6.191336 & -0.14347 \\
\hline $\mathrm{C}$ & -2.13367 & 4.711522 & -2.03257 \\
\hline $\mathrm{C}$ & -4.19464 & 6.105148 & -2.25542 \\
\hline $\mathrm{H}$ & -3.64349 & 6.694642 & 0.38774 \\
\hline $\mathrm{H}$ & -2.25351 & 5.611238 & 0.598955 \\
\hline $\mathrm{H}$ & -2.161 & 6.971335 & -0.55424 \\
\hline $\mathrm{H}$ & -2.43943 & 4.080739 & -2.88094 \\
\hline $\mathrm{H}$ & -1.52637 & 5.536448 & -2.43486 \\
\hline $\mathrm{H}$ & -1.48113 & 4.110957 & -1.38202 \\
\hline $\mathrm{H}$ & -3.59267 & 6.91426 & -2.70023 \\
\hline $\mathrm{H}$ & -4.57542 & 5.472002 & -3.07201 \\
\hline $\mathrm{H}$ & -5.06036 & 6.564723 & -1.7554 \\
\hline $\mathrm{C}$ & 2.86801 & -5.29207 & -0.00088 \\
\hline $\mathrm{C}$ & 2.302211 & -6.49239 & 0.431667 \\
\hline $\mathrm{C}$ & 0.952319 & -6.52062 & 0.797699 \\
\hline $\mathrm{C}$ & 0.184353 & -5.35792 & 0.735709 \\
\hline $\mathrm{C}$ & 0.752626 & -4.14556 & 0.306156 \\
\hline $\mathrm{C}$ & 2.094186 & -4.13175 & -0.06823 \\
\hline $\mathrm{C}$ & -0.17487 & -2.94552 & 0.264785 \\
\hline $\mathrm{C}$ & -0.64857 & -2.39174 & 1.563389 \\
\hline $\mathrm{C}$ & -2.13378 & -2.49256 & 1.804431 \\
\hline $\mathrm{C}$ & 0.125953 & -2.90397 & 2.704185 \\
\hline $\mathrm{O}$ & 1.304175 & -3.18589 & 2.700916 \\
\hline $\mathrm{O}$ & -2.88448 & -3.22873 & 1.191289 \\
\hline $\mathrm{C}$ & -1.14971 & -3.1439 & -0.88986 \\
\hline $\mathrm{O}$ & -0.64079 & -3.11664 & 3.824337 \\
\hline $\mathrm{C}$ & -0.34529 & -5.41213 & 4.558449 \\
\hline $\mathrm{C}$ & -0.08387 & -3.94403 & 4.848848 \\
\hline $\mathrm{O}$ & -2.74899 & -1.71109 & 2.739341 \\
\hline $\mathrm{C}$ & -2.51605 & -0.85259 & 4.973964 \\
\hline $\mathrm{C}$ & -2.1474 & -0.67653 & 3.515064 \\
\hline $\mathrm{H}$ & 3.923845 & -5.24968 & -0.27729 \\
\hline $\mathrm{H}$ & 2.9078 & -7.40029 & 0.4868 \\
\hline $\mathrm{H}$ & 0.494452 & -7.45303 & 1.138099 \\
\hline $\mathrm{H}$ & -0.86474 & -5.37591 & 1.042175 \\
\hline $\mathrm{H}$ & 2.539262 & -3.18851 & -0.37111 \\
\hline $\mathrm{H}$ & -0.58677 & -3.20178 & -1.83048 \\
\hline $\mathrm{H}$ & -1.68273 & -4.0913 & -0.7442 \\
\hline $\mathrm{H}$ & -1.91445 & -2.36966 & -0.96816 \\
\hline $\mathrm{H}$ & 0.018535 & -6.03641 & 5.390512 \\
\hline $\mathrm{H}$ & -1.42231 & -5.59784 & 4.426191 \\
\hline $\mathrm{H}$ & 0.180064 & -5.71784 & 3.641641 \\
\hline
\end{tabular}




$\begin{array}{llll}\mathrm{H} & 0.994753 & -3.75479 & 4.923869 \\ \mathrm{H} & -0.57198 & -3.6228 & 5.781411 \\ \mathrm{H} & -2.10317 & -0.02184 & 5.568116 \\ \mathrm{H} & -3.60959 & -0.86043 & 5.097883 \\ \mathrm{H} & -2.11349 & -1.79875 & 5.357872 \\ \mathrm{H} & -1.06234 & -0.66305 & 3.381071 \\ \mathrm{H} & -2.53795 & 0.284501 & 3.139291\end{array}$

